\title{
Images of Australian Odonata Wings
}

\author{
JOHN TANN (D) \\ Australian Museum Research Institute, \\ Australian Museum, 1 William Street, Sydney NSW 2010, Australia
}

\begin{abstract}
A recently finished project has created an openly accessible, high-resolution, photographic library of wings of Australian dragonflies and damselflies, order Odonata. The library is an open resource for identification and research. Both male and female wings of 318 species of dragonfly and damselfly have been photographed with a specialist set-up using identified museum collection material. In general, both wings were removed from the insect body to produce an image with a minimum of visual artefacts. Each resulting image shows a pair of right wings, a scale, an identifying taxonomic name and sex.
\end{abstract}

\section{Introduction}

Historically, there have been no readily accessible, good quality images of all Australian dragonflies and damselflies (Odonata), for identification purposes. Comparative morphology is arguably the most informative first step in any dependable identification process but it relies on high resolution, detailed imagery of diagnostically important structures across all known species. Ideally such imagery should be of the most typical representatives of a species (type specimens) or specimens that are expertly identified, and every image standardized in such a way that humans or human-assisted machines can consistently detect and process similarities and differences. It is possible that certain structures, thought to be diagnostically uninformative, are found to be much more useful when all species of a large group are assembled in the same orientation, format, and scale.

In the past, Odonata specimens in some of Australia's largest insect collections have been photographed at different times using a range of methods and images have been made available online. Such resources are not specifically images of wings or of any other particular structure. For example, the Australian National Insect Collection has imaged whole drawers of insect specimens, including dragonflies and damselflies (see Mantle et al., 2012). Another example, DigiVol, a volunteer program developed by the Australian
Museum, has been generating images of whole specimens together with their label-data to streamline registration and cataloguing (see DigiVol, 2020).

Such images add intellectual value to the specimens and to the collections that accommodate them ultimately leading to a better understanding of the Australian Odonata fauna. Intellectual value is significantly increased in direct relationship to confidence of identification - the more confident the identification, the more valuable the data.

The popularity of image sharing sites such as Flickr, and citizen science observation sharing sites such as iNaturalist, has resulted in a significant number of highquality images of live dragonflies and damselflies being made freely available. In 2020, iNaturalist held about 30,000 Australian observations of Odonata, $88 \%$ at Research Grade (identification agreed by two or more people) (iNaturalist, 2020); Flickr held about 20,000 images tagged as a dragonfly or damselfly in Australia (Flickr, 2020).

Wing morphology, especially venation, is not only diagnostically significant in taxonomy (e.g., Tillyard, 1917), but is used for demonstrating species variation (e.g., Stewart, 1982), for field identification (e.g., Theischinger, Hawking \& Orr, 2021), for understanding wing function and evolution (e.g., Salcedo et al., 2019), and other studies (e.g., phylogeny, Trueman, 2001). This project has created a resource for identification and research based on high-quality wing photographs. 

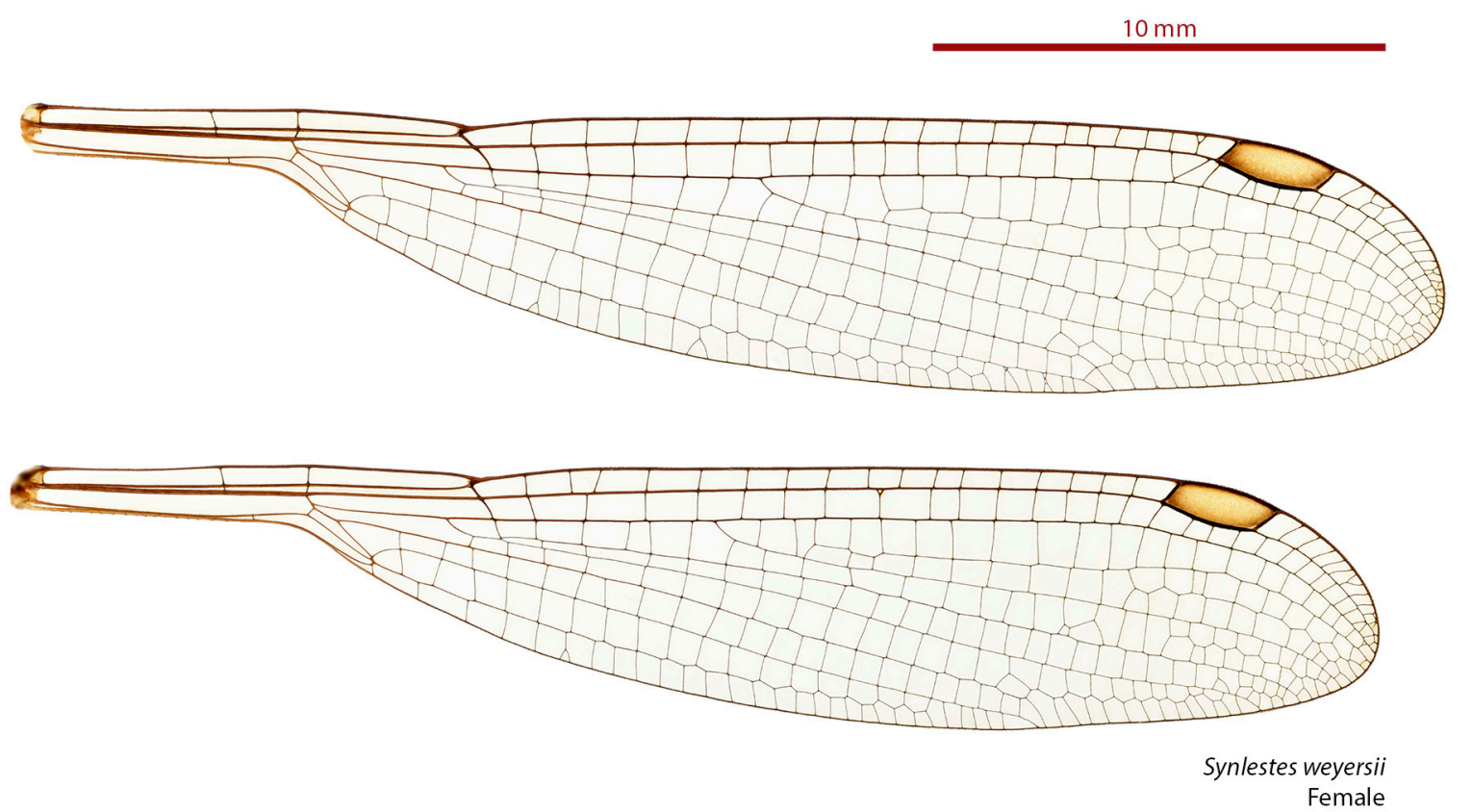

Figure 1. Image example: wings of a female Synlestes weyersii, a damselfly. This image and others are available in high-resolution, about $9000 \times 5000$ pixels $($ Tann, 2020a-e).

From 2013 to 2015 wings of Australian dragonflies and damselflies were photographed at the Australian Museum in Sydney, Australia. In that time, 318 of the approximately 324 known species of Australian dragonflies and damselflies were photographed and, with few exceptions, images were made of both male and female wings. Some species were not able to be photographed, either because of restricted access, rarity, or physical condition; for some species only one sex is known. This project drew from identified and vouchered specimens, either pinned or in envelopes, held at the Australian Museum, supplemented by specimens from four other Australian institutions: Queensland Museum in Brisbane, Western Australian Museum in Perth, Museum and Art Gallery of the Northern Territory in Darwin and Australian National Insect Collection in Canberra.

\section{Materials and method}

In order to create high resolution and standardized images, wings were removed from most specimens so they could be photographed without artefacts (see Why remove the wings? below). Dragonflies and damselflies specimens held in envelopes were generally cleaner, easier to work with, and required less reconstruction after photographing compared to pinned specimens.

\section{Technique}

A specimen in poor condition and with wings intact was selected. Many dragonfly and damselfly specimens in collections have broken bodies, separated abdomens or heads. Using this destructive technique - removing wings from an already-damaged specimen could be justified because it has contributed intellectual value from a mixed series of good and broken specimens. All specimens were registered, with few exceptions, and expert identification of each specimen was critical. Only one specimen at a time was processed before being returned to its drawer to avoid a mix-up. A whole-of-body photograph of the original specimen with all labels was made before further work, for reference and quality control; images of each reference specimen, including associated labels, have been published online (Tann, 2020e) and are freely accessible and expected to persist into the future. Both right wings were surgically removed microscopically using fine-pointed forceps. Occasionally, due to availability, left wings were used and their images artificially flipped (see Abnormal specimens below). Both wings were photographed independently using a good quality camera and customized rig. Wings and bodies were returned to their envelope. The wings of pinned specimens were glued to paper points and re-joined with their bodies on the same pin. Occasionally wings were photographed while still attached to the insect body; this was a sub-optimal method, used for rare and special specimens (see Visual artefacts below).

\section{Image capture equipment}

The following photographic equipment was used (with specimens staged on an inverted glass petri dish: $200 \mathrm{~mm}$ diameter, $37 \mathrm{~mm}$ high):

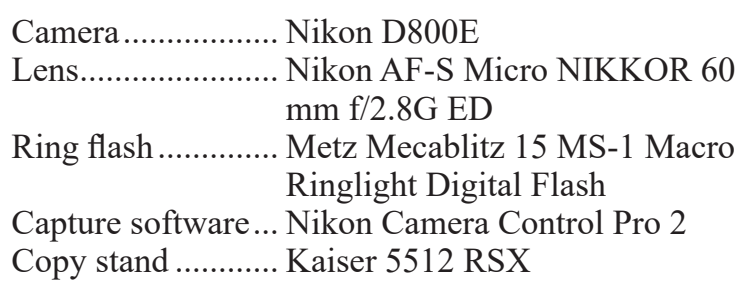

Camera Control Pro 2 software controlled the camera settings and shutter. The ring flash was set to trigger off the flash of the main camera. The software also captured photographic images directly to the controlling desktop computer. Forewings and hindwings were photographed separately and, during post-processing, combined in a single image of both wings. 


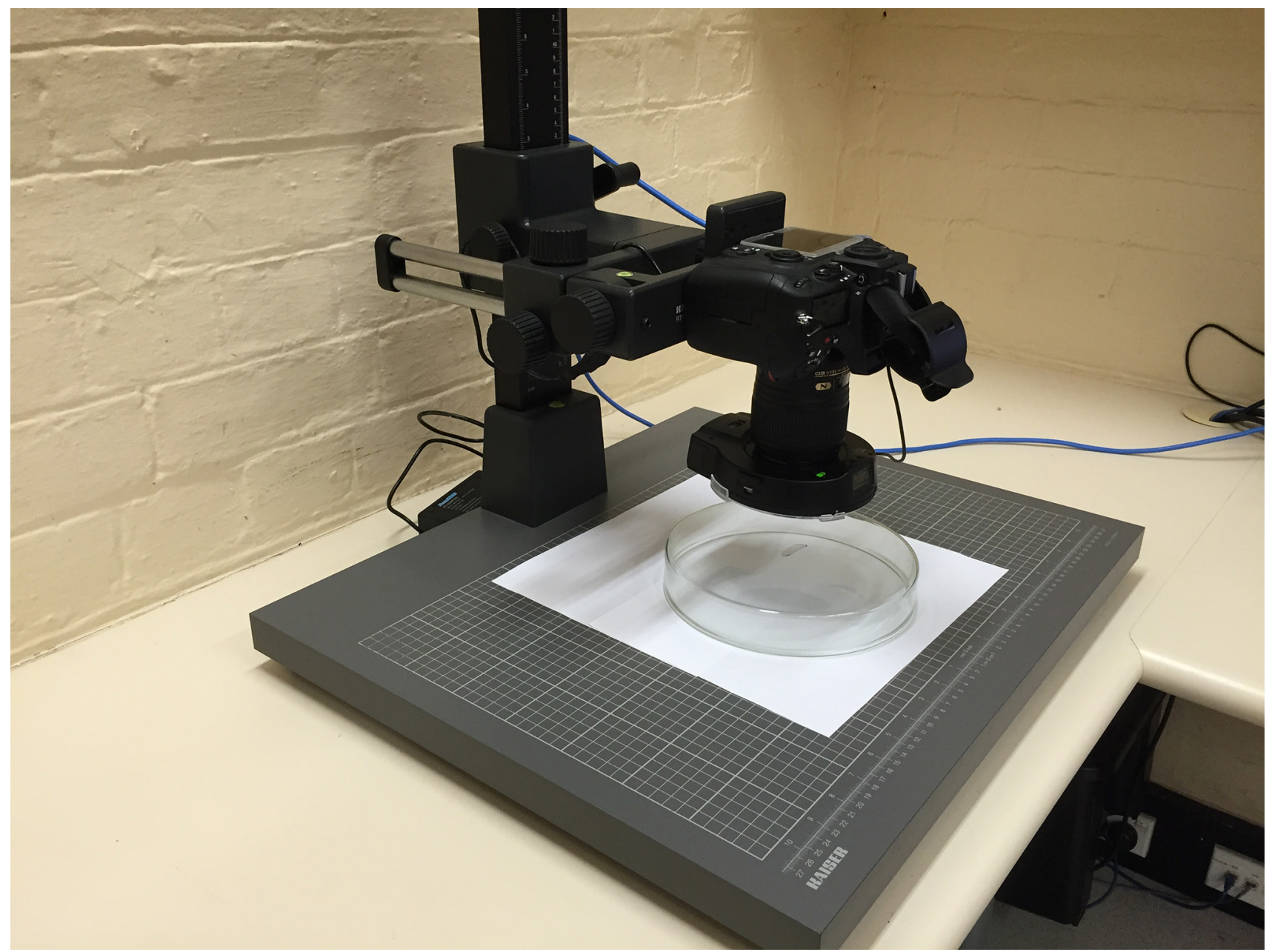

Figure 2. Photographic set-up for dragonfly and damselfly wings optimized to produce an image without shadows or visual artefacts.

\section{Equipment layout}

The photographic set-up was optimized to consistently capture high-resolution images of wings, minimizing visual artefacts. Dragonfly and damselfly wings are usually transparent, sometimes with colour, and have a length ranging from c. $15 \mathrm{~mm}$ for the smallest damselfly to c. 75 $\mathrm{mm}$ for the largest dragonfly.

The camera was mounted vertically above the specimen pointing down. A copy-stand-mount allowed vertical adjustment of the height of the camera which was consistent for almost all dragonfly and damselfly wings. Some very large dragonflies with long wings required a higher camera position.

A large petri dish was found to be an ideal photographic platform for minimizing visual artefacts. It was used upside down - the mouth of the petri dish was face down and its flat glass base was off the copy stand platform supporting a wing specimen at $37 \mathrm{~mm}$ above a clean white sheet of paper. The selected height between object and background produced an image with even lighting and without obvious shadow.

Equipment set-up for photographing specimens where wings could not be removed is discussed below (see Visual artefacts below).

\section{Camera settings}

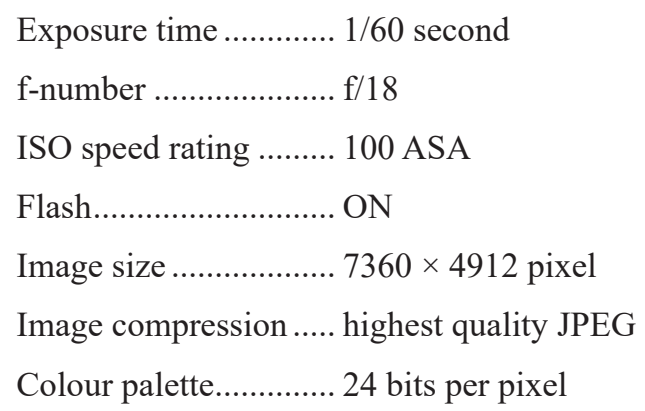

The compression format, JPEG, was chosen to give good results without visibly compromising image quality. Without compression, file sizes were overly large and consequently unwieldy to store, transfer and manipulate.

\section{Object position}

Height of wing above white background.... $37 \mathrm{~mm}$ Front of lens to object distance. $45 \mathrm{~mm}$

Each wing was photographed separately. 
Figure 3. Photograph of a single wing. Original photo of the forewing of a female Austrogomphus bifurcatus as recorded using the above camera rig. Note there are no shadows and a reasonably even near-white background. This photo has been reduced in size for publication.

\section{Image post-processing}

Photoshop graphics editor, published by Adobe ${ }^{\circledR}$, was used for generating a standardized image of both right wings, visual removal of dust, and visual repair of small areas of wing damage.

Lightroom (image organization and manipulation software published by Adobe ${ }^{\circledR}$ ) was used for managing photographs, adding metadata, and preparing a final version of each image.

ExifTool (open-source software used for reading, writing and manipulating image metadata-Harvey, 2020) was used for adding georeferences and other Exif metadata (a standard that specifies formats for image metadata used by digital cameras).

\section{Final image}

Each final image shows a pair of wings on a white background with a scale bar and identification.

Size $.9008 \times 5067$ pixel (ratio 16:9)

Colour palette ...24 bits per pixel

Scale Most dragonfly and damselfly wing images were photographed at a resolution of 1357 pixels per centimetre, or about $7 \mu \mathrm{m}$ per pixel. This scale was a product of the object to camera distance that was selected early in the project. Some larger wings were photographed at a resolution of 1000 pixels per centimetre, or about $10 \mu \mathrm{m}$ per pixel.

For greater usability, two sets of images were created: one set with as-shot resolution of 1357/1000 pixels per centimetre; ${ }^{1}$ a second set where final wing image size was adjusted for best fit to one of six fixed resolutions. ${ }^{2}$ For each image a scale bar was added.
Table 1. Image display resolution. One set of images was scaled to fit one of six resolution bands.

\begin{tabular}{lll}
\hline $\begin{array}{l}\text { resolution pixels } \\
\text { per centimetre }\end{array}$ & $\begin{array}{l}\text { horizontal and } \\
\text { vertical scale }\end{array}$ & $\begin{array}{l}\text { colour of } \\
\text { scale bar }\end{array}$ \\
\hline 1000 & $73.7 \%$ & blue \\
1357 & $100 \%$ & black \\
2000 & $147.4 \%$ & blue \\
2500 & $184.2 \%$ & brown \\
4000 & $294.8 \%$ & blue \\
5000 & $368.5 \%$ & blue \\
\hline
\end{tabular}

There was no standard way to embed the image scale into Exif metadata. Exif parameters such as XResolution and YResolution, even if initially set correctly, were able to be manipulated subsequently and misleadingly by viewing software.

To ensure final wing images maintained their correct identification, the taxonomic name, according to the Australian Faunal Directory, ${ }^{3}$ and sex, male or female, were added to the lower right corner of each image. A filename was allocated to readily convey species, sex and scale. For example: "Austroaeschna hardyi female 1357.jpg".

\section{Abnormal specimens}

Occasionally undamaged right wings were not available and left wings were used as substitutes. When this occurred a mirror image of the left wing was created in software so as to appear like a right wing. This modification was noted in the image metadata. 


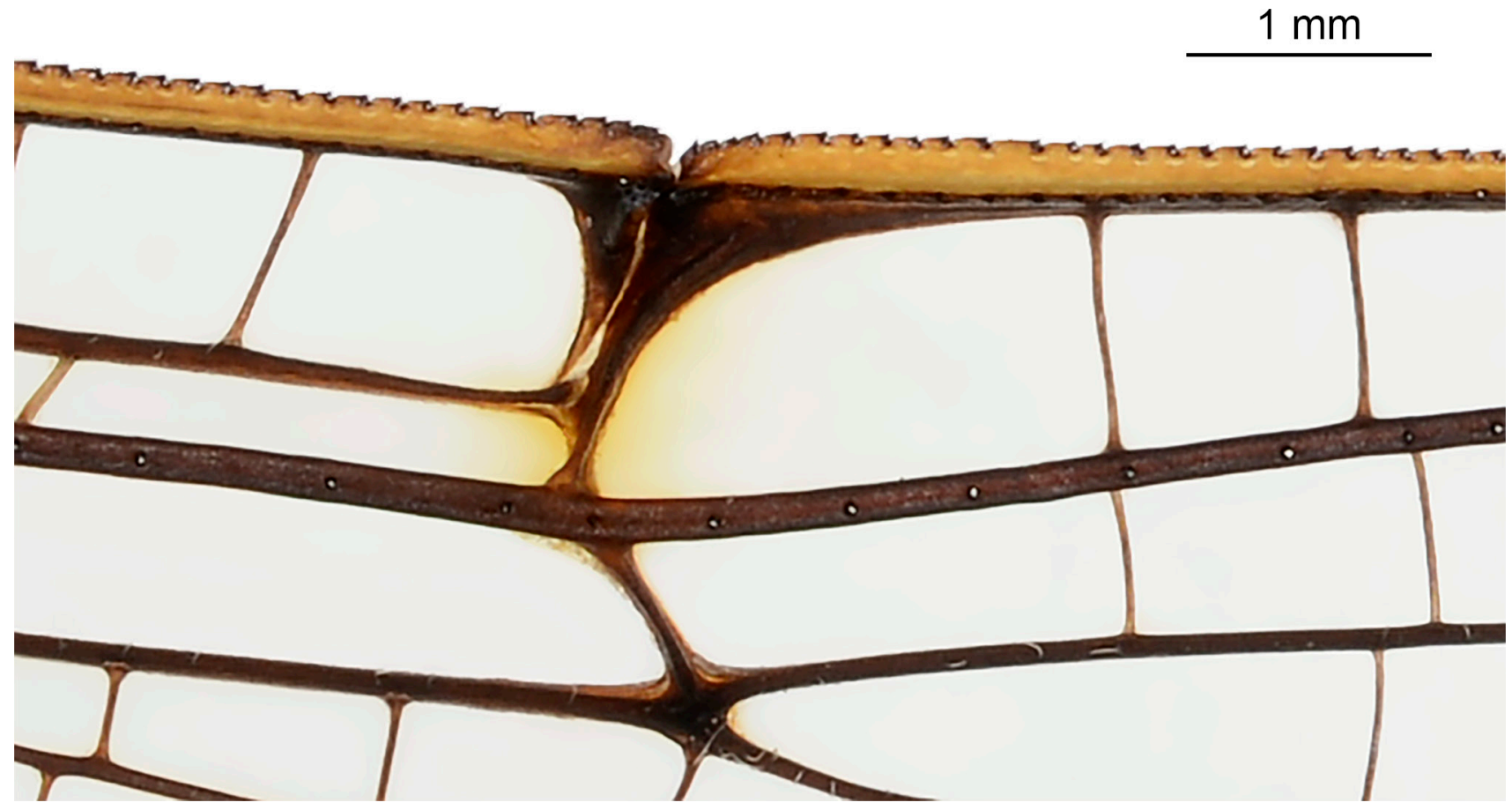

Figure 4. Image detail clearly showing saw-tooth ridges on the leading edge of the forewing of a male Green Emperor, Anax gibbosulus. Each ridge is about $150 \mu \mathrm{m}$ long. The structure in the upper central part of this photo is the nodus. This wing was photographed at a resolution of 1357 pixels per centimetre. Many dragonfly and damselflies have microstructure on their wing veins clearly visible in this set of high-resolution photographs.

\section{Results}

\section{Presentation of images}

All images have been published online on open access platforms:

1 On figshare, images can be viewed individually or downloaded as a series (Tann, 2020a-e).

2 On Flickr, each wing image is accompanied by a caption. Other metadata for each image, such as specimen location on a map, tags, comments, and a link to the collection data, are shown on each page (Tann, 2017a,b).

3 On Wikimedia Commons. Wikipedia in turn displays wing images on the content page of each Australian dragonfly and damselfly (see, for example, Wikipedia, 2020).

4 On the Atlas of Living Australia as a Dataset Resource. Wing images and specimen images are readily viewable as a collection (Tann, 2020f).

Each image is licensed under a Creative Commons Attribution 4.0 International License (CC BY 4.0), which permits unrestricted use, distribution, and reproduction in any medium, provided the original author and source are credited.

Image sets are presented as two sub-sets, Anisoptera or dragonflies proper, and Zygoptera, or damselflies.

\section{Metadata}

Images hold extensive embedded metadata:

1 Metadata associated with the camera and photograph — embedded as Exif metadata

2 Metadata about the specimen - embedded as IPTC metadata $^{4}$

- Registration number

- Institution

- Taxonomic name, according to the Australian Faunal Directory (2020)

- Georeference, extracted from any occurrence data held by the Atlas of Living Australia ${ }^{5}$

- Sex

3 Title and caption metadata - embedded as IPTC metadata. Caption information includes common name, taxonomic name, collection and specimen registration number.

4 Additional metadata enabling image-filtering are embedded as keywords:

- Sex

- Country

- Taxonomy

- Image descriptors 


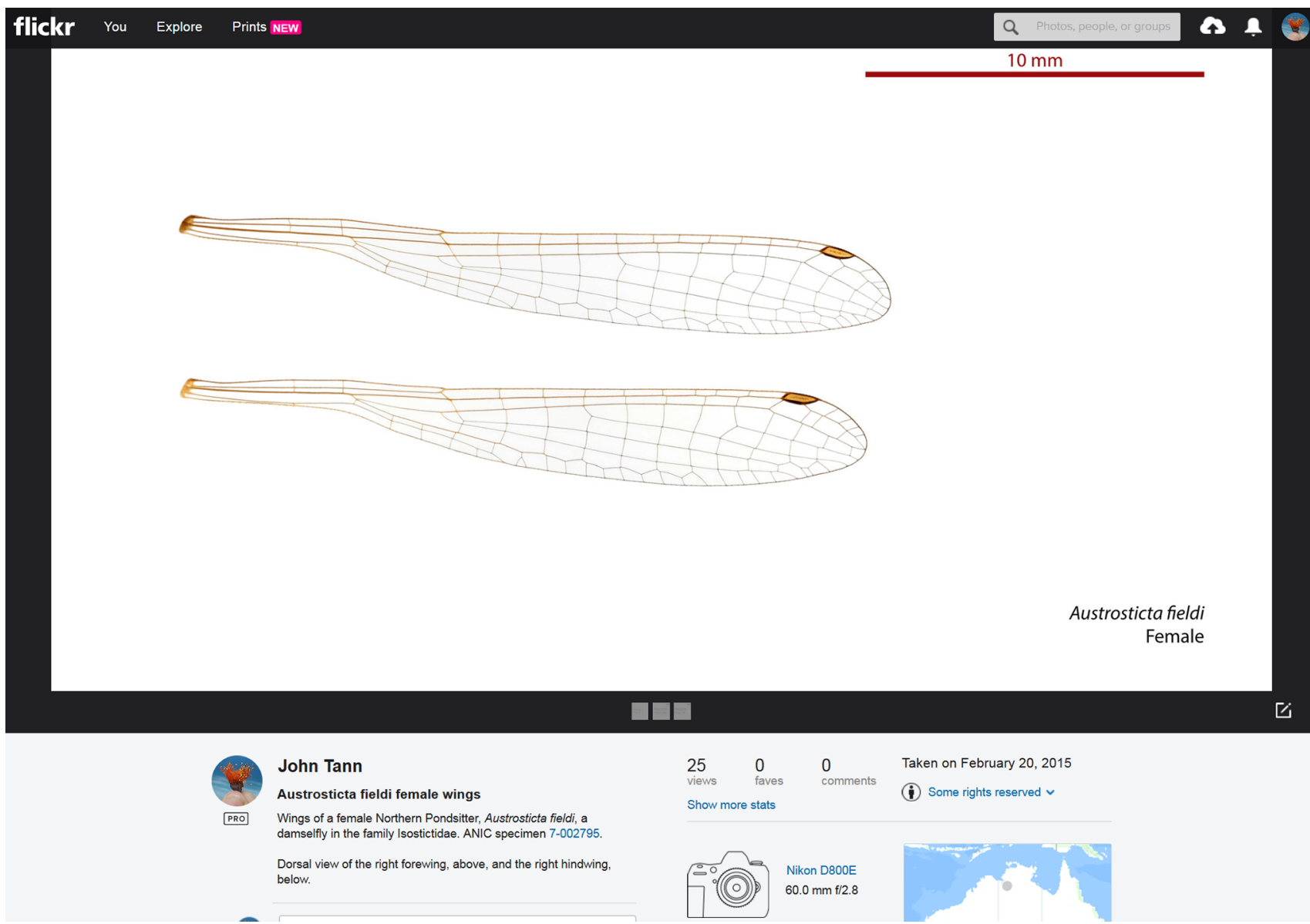

Figure 5. Example of a Flickr image with caption. The registration number link takes the user to its occurrence record on the Atlas of Living Australia.

\section{Links}

Due to the efforts of museum staff in the past, occurrence data for a large number of museum dragonfly and damselfly specimens in Australia is publicly accessible through the Atlas of Living Australia (ALA). ${ }^{6}$ ALA occurrence records for specimens used in this project are linked in the Index (Tann, 2020e, see also Appendix 3 and 4).

On the figshare platform male and female wing images of each species are published with a DOI, a digital object identifier. A DOI is also a persistent object identifier offering a degree of permanence ${ }^{7}$. Full-size images of wings and their reference specimens on figshare are linked in a large published table: the Index (Tann, 2020e).

\section{Discussion}

\section{Why remove the wings?}

In order to capture consistently high-quality images, in a single, shallow, plane of view, a wing needed to be photographed separated from its body. Having an insect body, another wing, or a leg obstructing the field of view or casting shadow are undesirable visible artefacts. Wings are preserved with their relevant specimen after photography.

\section{Visual artefacts}

For those pinned specimens which had wings that could not be removed, a temporary rig was made to minimize visual interference.
The effect of the body shadow could be reduced by changing the object height above its background. However, with increasing height the white background changed to grey, with a consequent loss of image fidelity and consistency.

Insect specimens in museums are stiff, brittle and fragile; their long-term preservation, intact and undamaged, is of obvious and great importance. On rare occasions, however, when a surplus of conspecific specimens are at hand, or when the significance of the knowledge likely to be gained is great, will a curator allow dissection or manipulation of a specimen. Such procedures are conditional upon every effort being made to secure all removed fragments or body parts so that they may be preserved with the host body in collections. The technique of wing analyses by highresolution photography results in the removal, but not the loss, of wings.

Odonata legs, heads and abdomens break easily. Wings cannot be bent and legs cannot be pushed aside without damage.

\section{Wing flatness}

Dragonfly and damselfly wings are not absolutely flat. Wings with irregular, undulating surfaces were sandwiched between two glass plates a few millimetres apart to achieve a moreor-less flat plane. This technique allowed uneven features to be imaged without crushing the wing. This method was, however, impractical on those occasions when wings could not be removed from their bodies. 


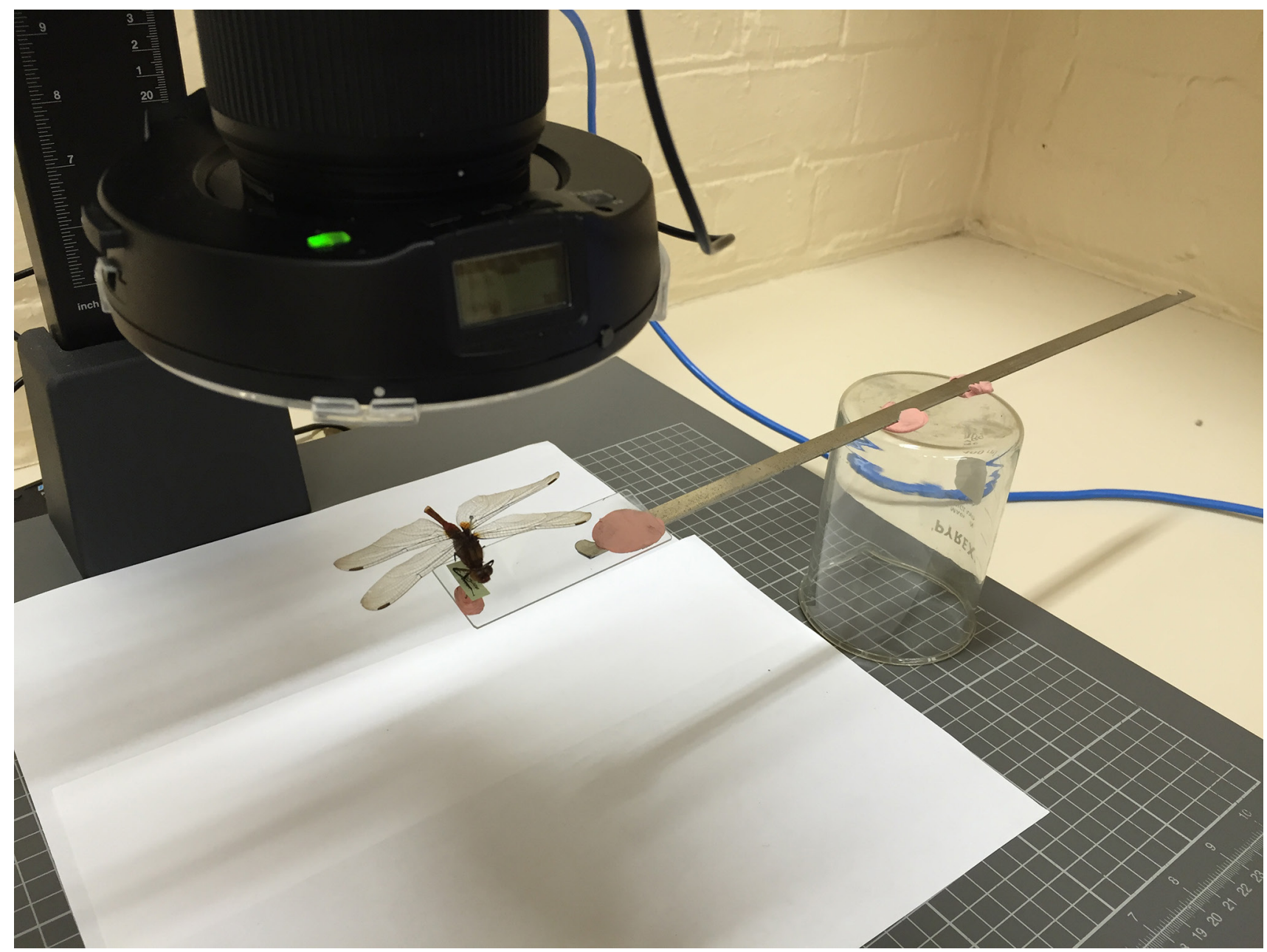

Figure 6. Photographic set-up for dragonfly and damselfly wings where the wings remained attached to their pinned body. The rig itself did not create obvious visual artefacts.

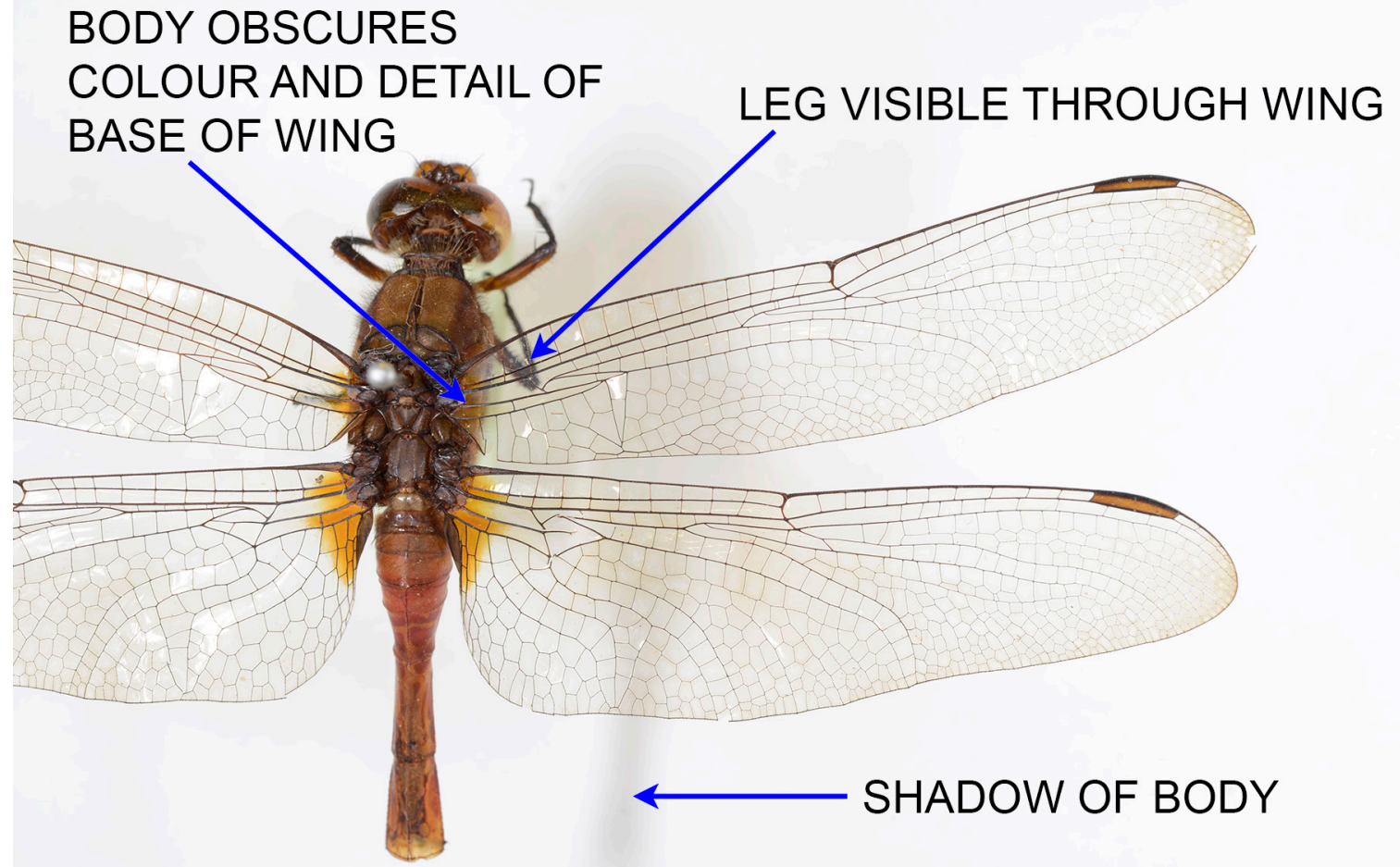

Figure 7. Photo showing three artefacts when a wing remains attached to its body. 


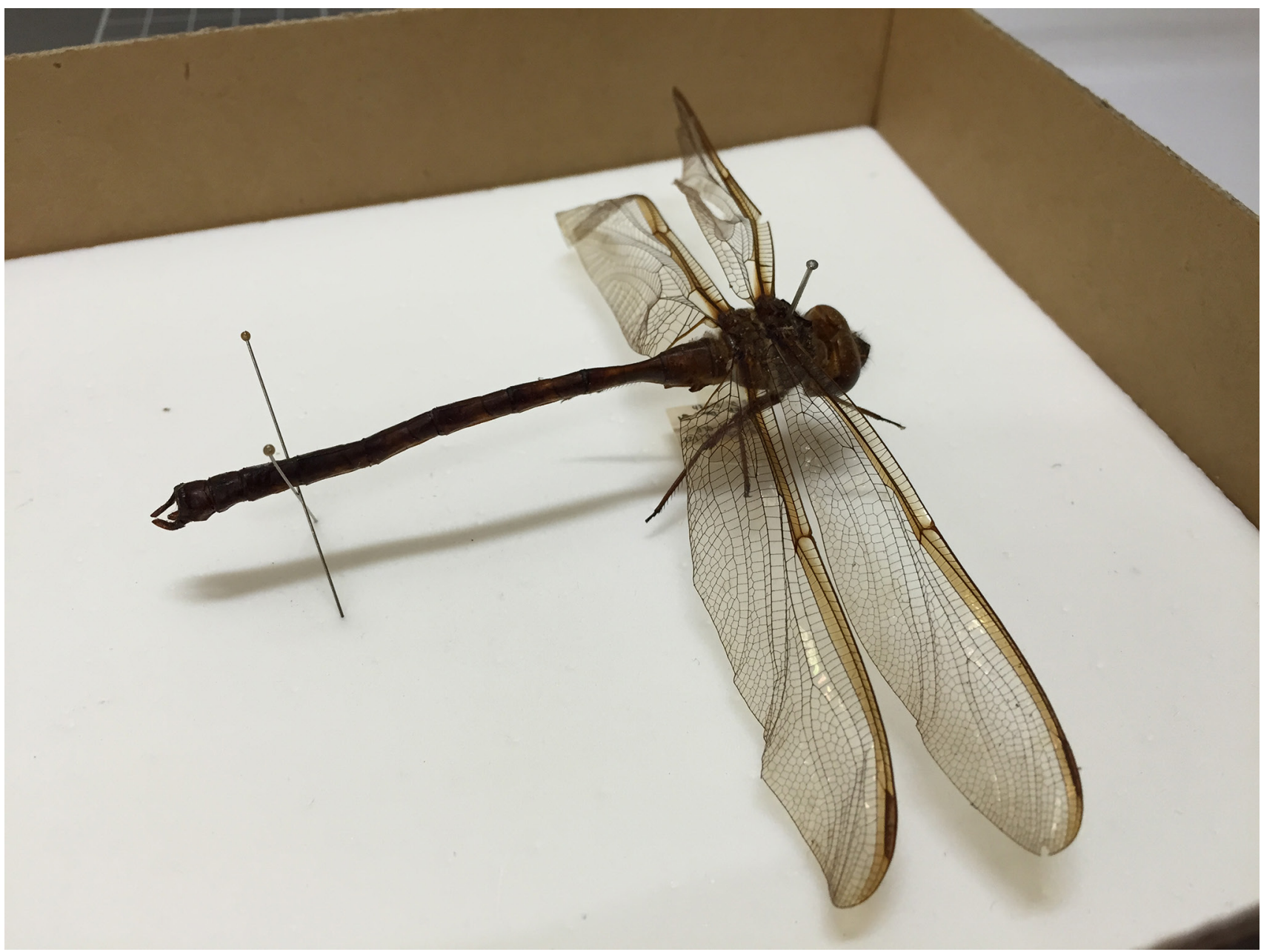

Figure 8. Male Austrophlebia subcostalis, a large dragonfly with wings that are not flat. Details are lost when photographing unflattened wings. Note also for this specimen that the two right wings do not lie in the same plane; see section: Overlapping and out-of-plane wings.

\section{Overlapping and out-of-plane wings}

Dragonfly and damselfly specimens are sometimes pinned with their wings overlapping. For those specimens, a clear wing photo was impossible.

Some dragonflies and damselflies had been pinned with their wings lying in different planes (see, for example, the photo of Austrophlebia subcostalis, Fig. 8). To reproduce a wing shape accurately, a photograph was always taken orthogonal to the wing plane. Unfortunately, out-of-plane wings would sometimes overlap in a photograph, making the resulting photograph unacceptable for this project.

\section{Photoshop as a surrogate for a good photograph}

Photoshop, or other image processing software, could be used to improve substandard images. With effort, some shadowing could be lightened, some artefacts could be covered over, and some flaws repaired. However, Photoshop had difficulty when colours were similar, such as distinguishing wing veins from body colour, and could not, of course, resurrect features hidden by another shape.

It was important that the complete dataset of dragonfly and damselfly wing images offered here be accurate and faithful reproductions of real wings. As such this imagedataset provides the basis of a detailed image-analysis research project. Using Photoshop to fill in the blanks, and estimate colours, shades, and subtle features was considered to be not good practice. In the course of this project about 10 specimens were photographed with their wings attached. Due to the extra difficulties of working with attached wings, each specimen took about half a day to photograph and two full days processing with Photoshop to produce a less-than-optimal result. Early in this project it became clear that in order to produce a satisfactory photograph, wherever possible wings would need to be removed.

Nonetheless all wing images were manipulated to a greater or lesser extent by Photoshop and Lightroom. Lightroom, being the last software to alter the image, is mentioned in the image metadata. 


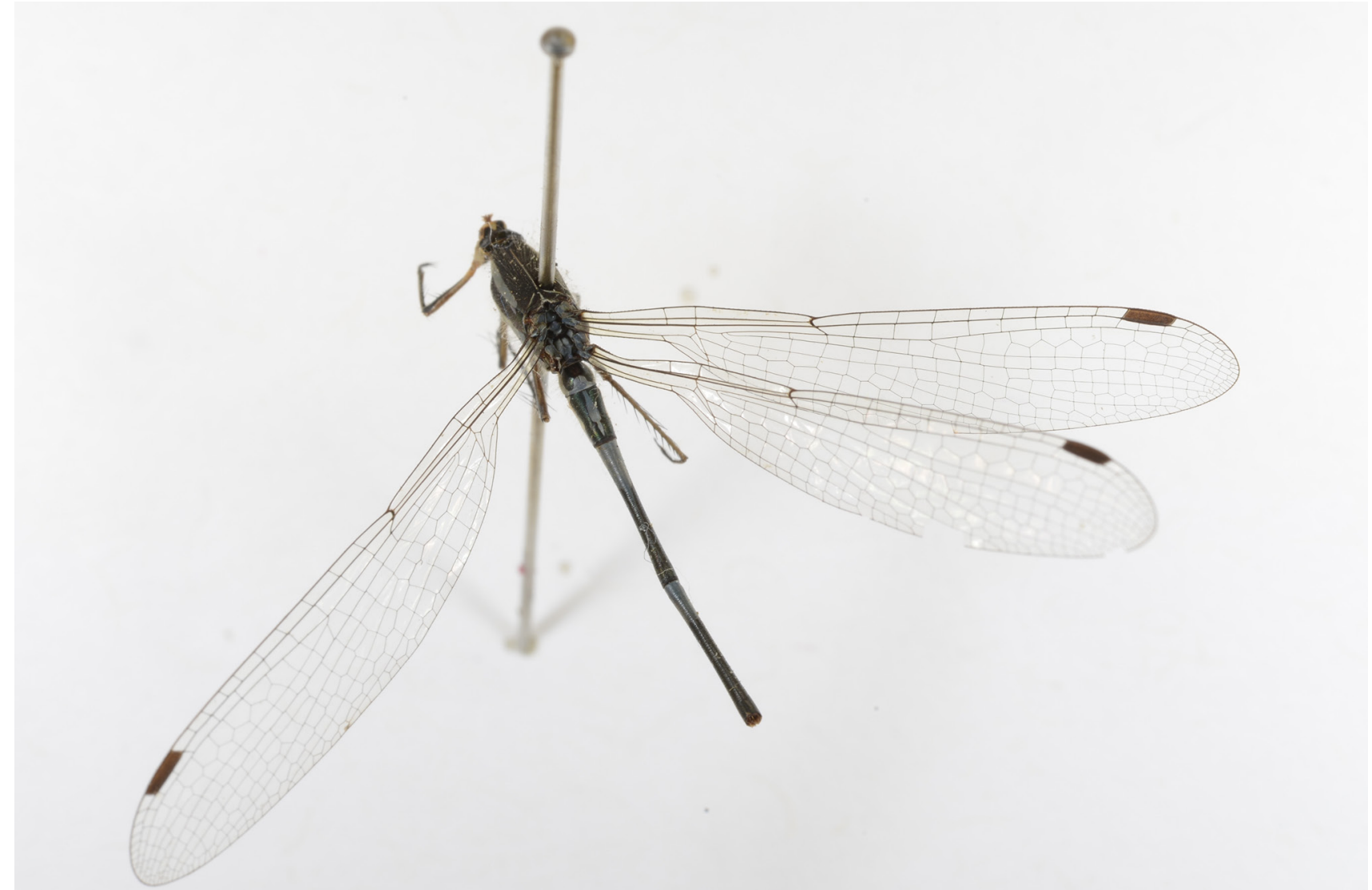

Figure 9. Damselfly pinned with its wings overlapping.

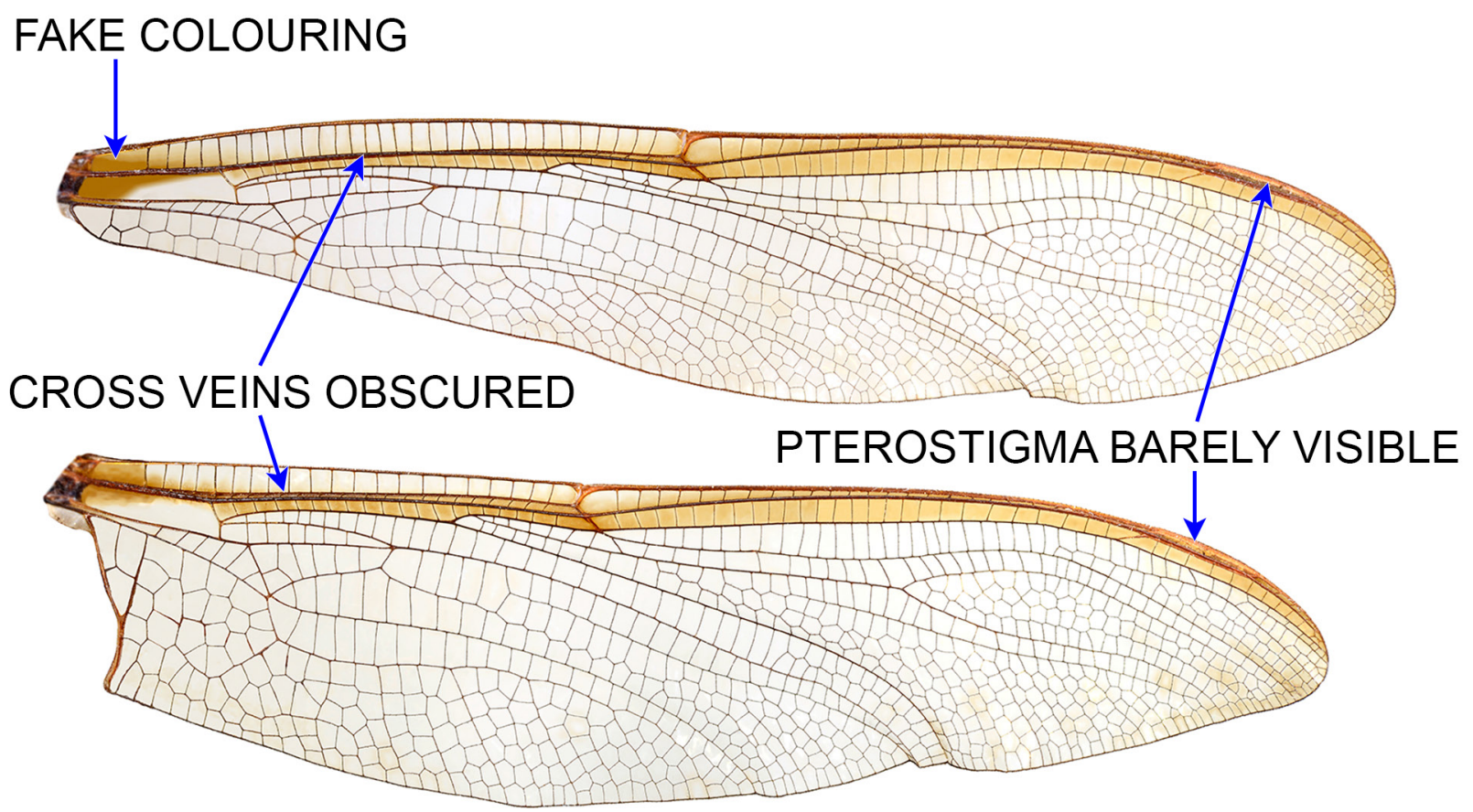

Austrophlebia subcostalis

Male

Figure 10. Processed image of wings photographed while still attached to a body. Parts of each wing were obscured. Extensive Photoshop treatment produced only a second-class image and introduced artefacts of shade and colour not apparent in the specimen. 
Acknowledgements. This project was undertaken in the Entomology department of the Australian Museum with access to its extensive collection of Australian Odonata. Photographic equipment was provided through the DigiVol program at the museum thanks to Paul Flemons and Rhiannon Stephens. Entomology staff were always helpful, and a big appreciation goes to Jacquie Recsei, Russell Cox, Derek Smith and Shane McEvey for their advice, assistance and support.

Other museums and collections contributed specimens to this project. Thanks should go to Beth Mantle, Nicole Fisher and David Yeates at Australian National Insect Collection, Nik Tartanic at Western Australian Museum, Susan Wright at Queensland Museum and Gavin Dally at Museum and Art Gallery of the Northern Territory.

\section{Endnotes}

1 Complete set of wing images reduced in size for publication in Appendixes 1 and 2, also available at full size on figshare (Tann, 2020c,d)

2 Complete set of scaled wing images available on figshare (Tann, 2020a,b)

3 The Australian Faunal Directory, AFD, is a catalogue of taxonomic information about all animal species known to occur in Australia. It includes the nomenclature and taxonomy of species including valid names (AFD, 2020).

4 An IPTC header follows a metadata standard for images. It has been defined by the International Press Telecommunications Council, a global standards body for news media.

5 The Atlas of Living Australia (ALA) is an online repository for sharing information about Australian plants and animals.

6 In 2020 the Atlas of Living Australia held about 40,000 museum records of Odonata $(A L A, 2020)$.

7 A Digital Object Identifier (DOI) is a persistent identifier for use on digital networks. It has a degree of permanence and promotes better interoperability across science now and into the future (DOI, 2020).

\section{References}

AFD. 2020. Australian Faunal Directory. [Accessed July 2020]. https://biodiversity.org.au/afd/home

ALA. 2020. Atlas of Living Australia. [Accessed July 2020]. https://www.ala.org.aul

Creative Commons. 2020. [Accessed July 2020]. https://creativecommons.org/

DigiVol. 2020. [Accessed July 2020]. https://australian.museum/get-involved/citizen-science/digivol/

DOI. 2020. [Accessed July 2020]. https://www.doi.org

Flickr. 2020. Flickr. [Accessed July 2020]. https://www.flickr.com/

Harvey, P. 2020. ExifTool. [Software]. Retrieved from metacpan. org.

https://metacpan.org/pod/distribution/lmage-ExifTool/exiftool

iNaturalist. 2020. iNaturalist. [Accessed July 2020]. https://www.inaturalist.org/

Mantle, B.L., J. La Salle and N. Fisher. 2012. Whole-drawer imaging for digital management and curation of a large entomological collection. ZooKeys 209: 147-163. https://doi.org/10.3897/zookeys.209.3169

Salcedo, M. K., J. Hoffmann, S. Donoughe, and L. Mahadevan. 2019. Computational analysis of size, shape and structure of insect wings. Biology Open 2019 8: bio040774. https://doi.org/10.1242//bio.040774

Stewart, W. E. 1982. An analysis of geographic variation of the adults of the Australian genus Diphlebia Selys (Odonata: Amphipterygidae). Australian Journal of Zoology 30(3): 433-460. https://doi.org/10.1071/ZO9820435

Tann, J. 2017a. Dragonfly wings. [Accessed July 2020]. https://www.flickr.com/photos/31031835@No8/albums/72157684642873975

Tann, J. 2017b. Damselfly wings. [Accessed July 2020]. https://www.flickr.com/photos/31031835@N08/albums/72157684003991555

Tann, J. 2020a. Wings of Australian Odonata-Volume 1. Anisoptera.

https://doi.org/10.6084/m9.figshare.11845230

Tann, J. 2020b. Wings of Australian Odonata-Volume 2. Zygoptera. https://doi.org/10.6084/m9.figshare. 12579959

Tann, J. 2020c. Wings of Australian Odonata-Volume 3. Anisoptera fixed scale.

https://doi.org/10.6084/m9.figshare.12612038

Tann, J. 2020d. Wings of Australian Odonata-Volume 4. Zygoptera fixed scale. https://doi.org/10.6084/m9.figshare.12612041

Tann, J. 2020e. Wings of Australian Odonata-Index. https://doi.org/10.6084/m9.figshare.11840013

Tann, J. 2020f. Wing images of Australian dragonflies. Atlas of Living Australia Data Resource, dr16455. [Accessed October 2020].

https://collections.ala.org.au/public/showDataResource/dr16455

Theischinger, G., J. Hawking, and A. Orr. 2021. The Complete Field Guide to Dragonflies of Australia. Second edition. Melbourne: CSIRO Publishing. ISBN 978-1-48-631374-7

Tillyard, R. J. 1917. The Biology of Dragonflies (Odonata or Paraneuroptera). Cambridge Zoological Series. Cambridge. University Press. [Accessed through Biodiversity Heritage Library, July 2020]. https://doi.org/10.5962/bhl.title.35170

Trueman, J. 2001. Evolutionary riddles. In Dragonflies of the World, ed. J. Silsby, pp. 185-190. Collingwood: CSIRO Publishing. ISBN 978-0-64-310087-9

Wikipedia. 2020. Diphlebia euphoeoides, an example of use of, and accessibility to, images of this project. [Accessed July 2020]. https://en.wikipedia.org/wiki/Diphlebia euphoeoides 


\section{Appendix 1-Anisoptera wings}

Photographs of female and male wings of 207 species of Australian dragonfly (Anisoptera). Each image shows a pair of right wings, a scale, a taxonomic name and sex. A link is provided in Appendix 3 to a high-resolution photograph for each species.

Images presented below are low resolution "thumbnails" providing a visual ready-reckoner and quick guide to more detailed imagery. The entire photographic library at highest resolution is openly accessible to view or download from figshare as either individual images or as a complete set of Australian Odonata wing images (Tann, 2020a-d).

With few exceptions all images are presented here at a consistent fixed scale for ready comparison between species. However, in order to present both the smallest and largest wings conveniently, the wings of six species of the largest Australian dragonflies are shown at a reduced scale, recognizable by a blue scale bar. 


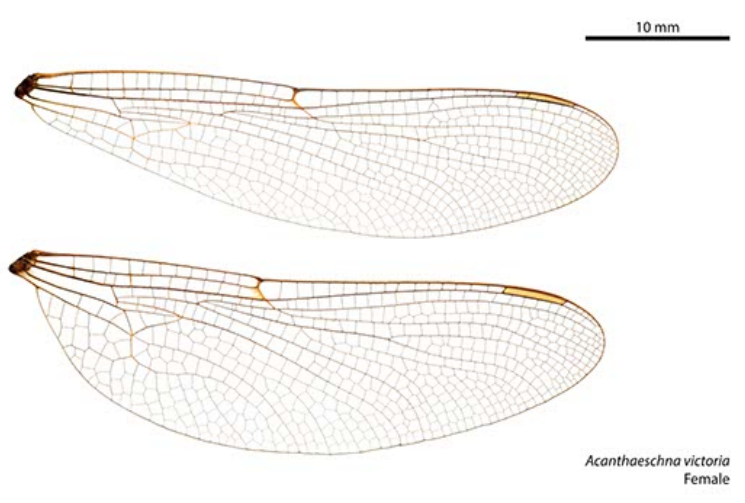

Acanthaeschna victoria female

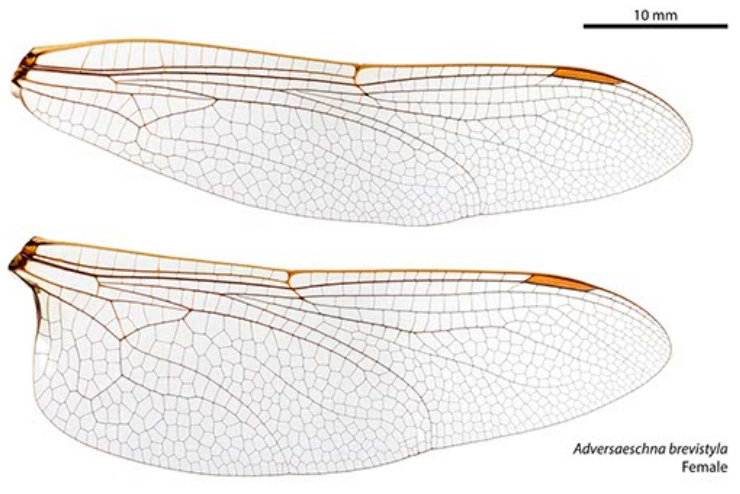

Adversaeschna brevistyla female

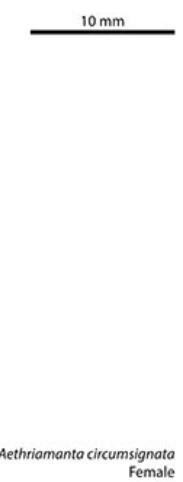

Aethriamanta circumsignata female

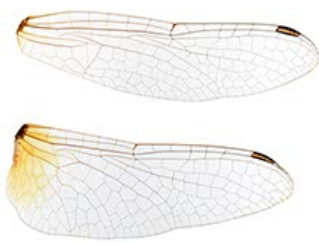

Aethriamanta nymphoeae

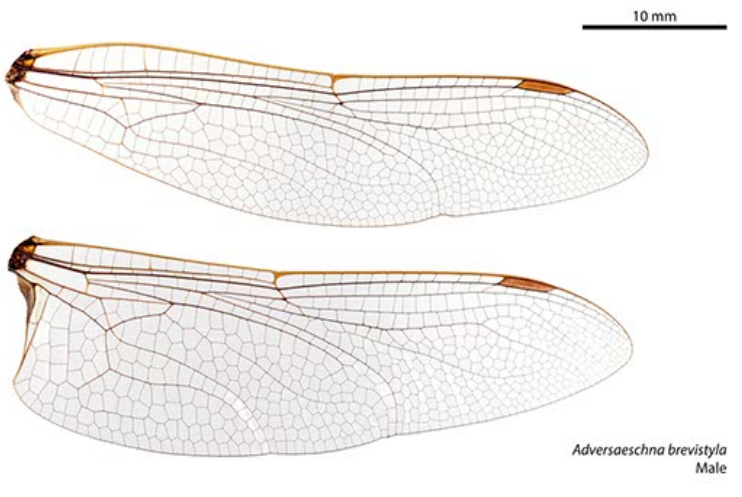

Adversaeschna brevistyla male

Acanthaeschna victoria male

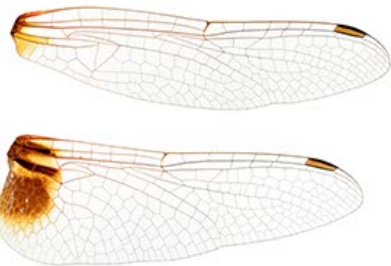

Aethriamanta circumsignata
Male

Aethriamanta circumsignata male

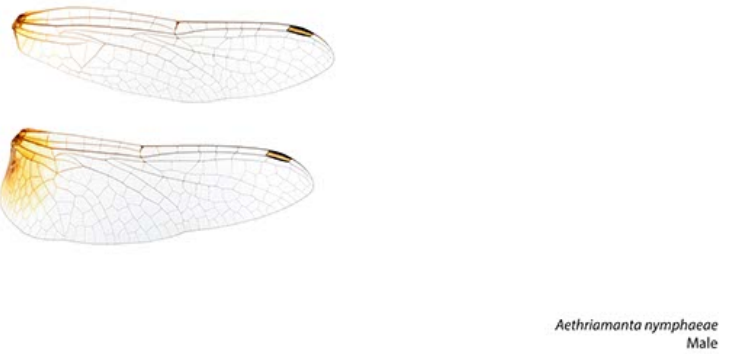




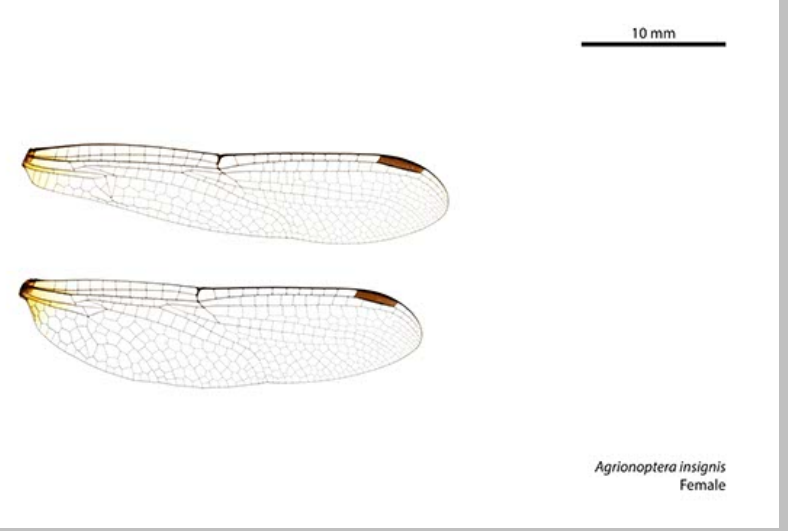

Agrionoptera insignis female

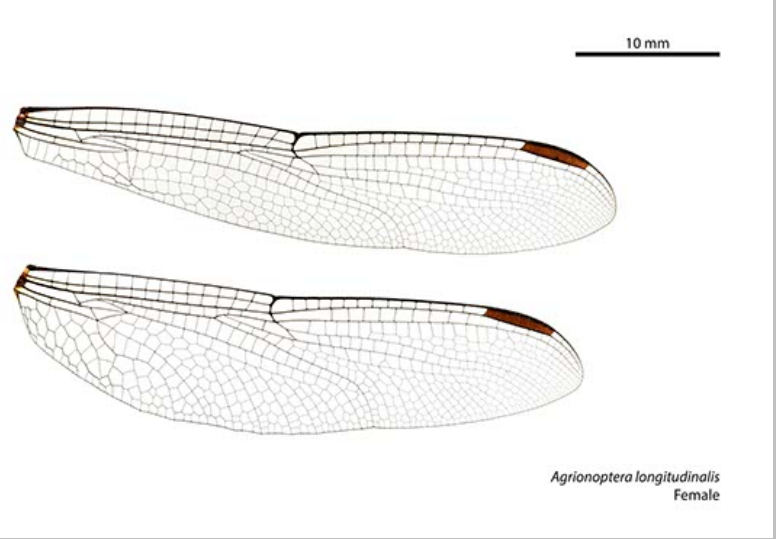

Agrionoptera longitudinalis female

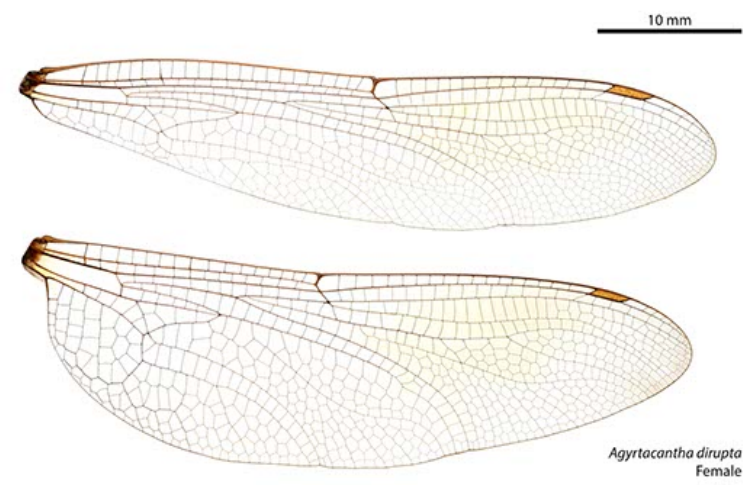

Agyrtacantha dirupta female

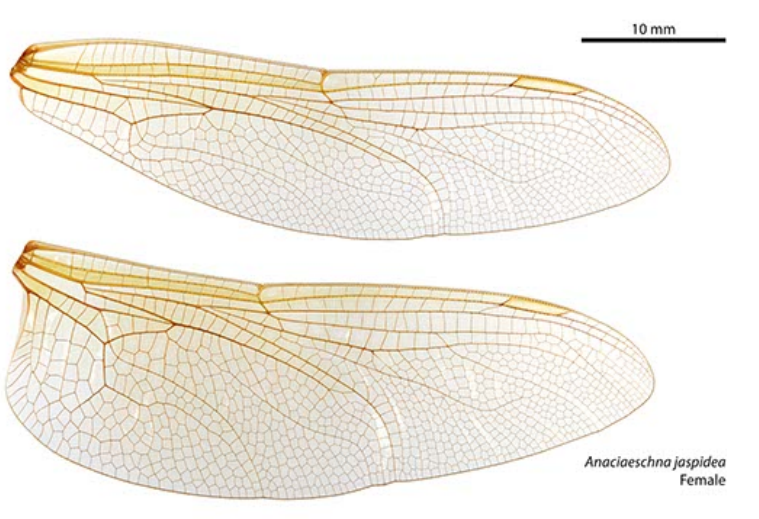

Anaciaeschna jaspidea female

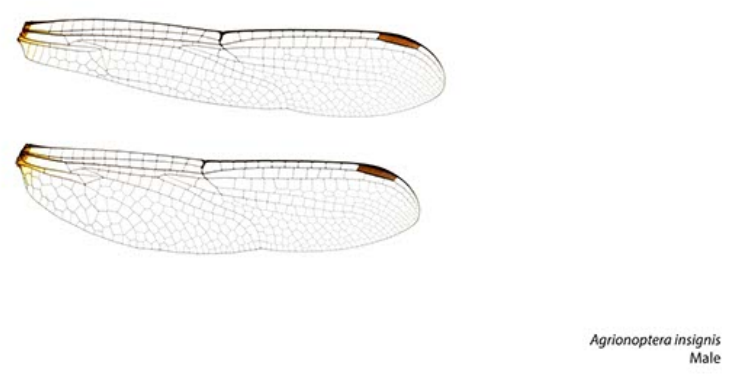

Agrionoptera insignis male

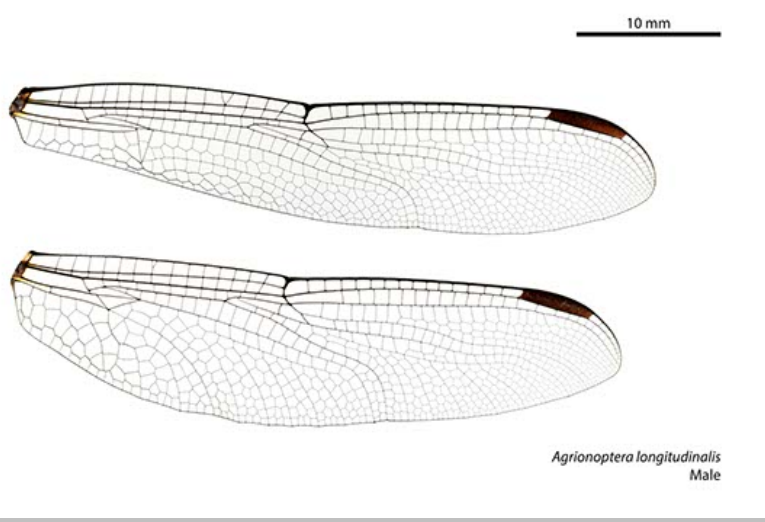

Agrionoptera longitudinalis male
Agyrtacantha dirupta male

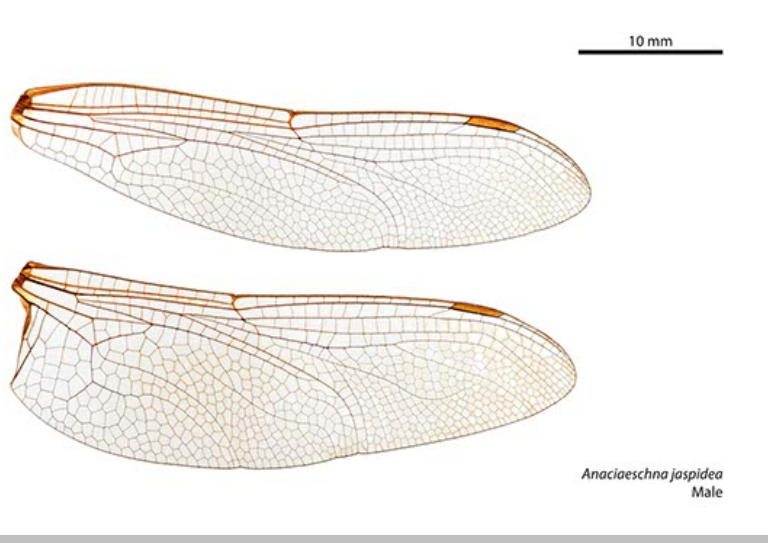

Anaciaeschna jaspidea male 

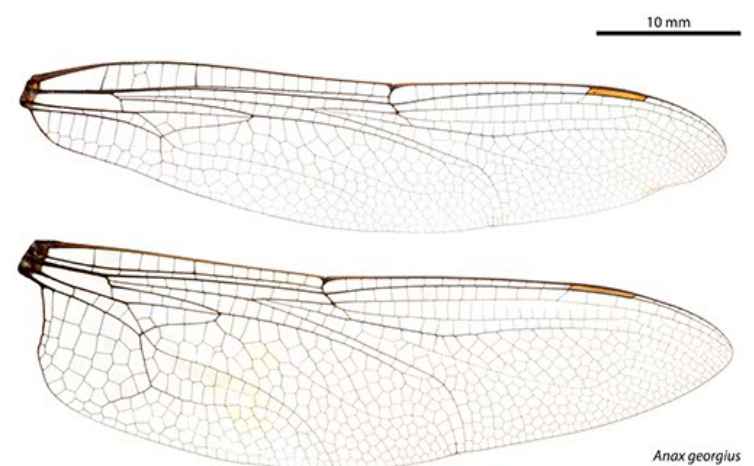

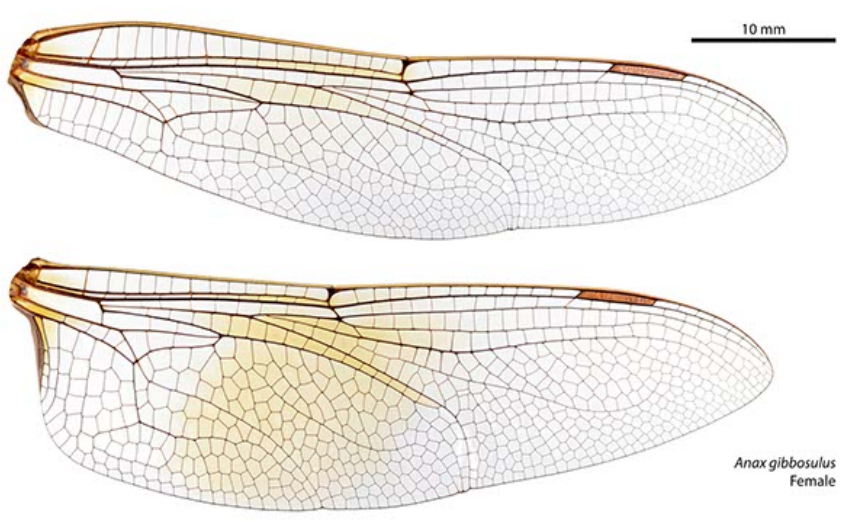

Anax gibbosulus female

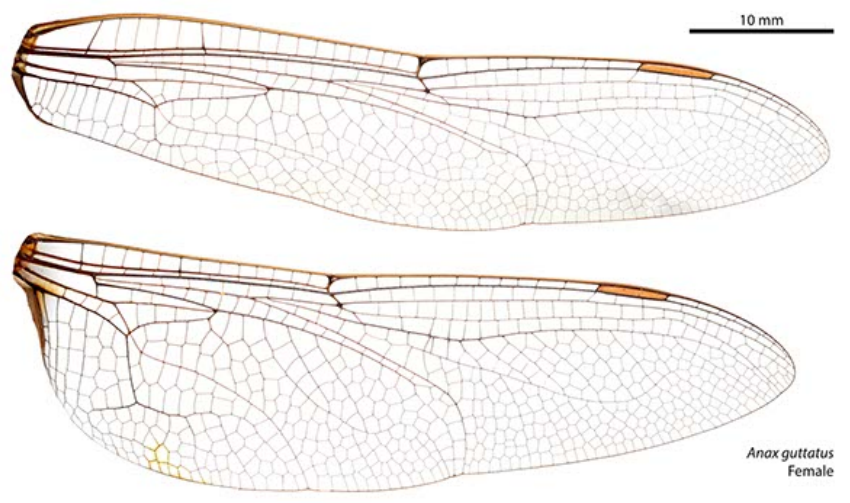

Anax guttatus female
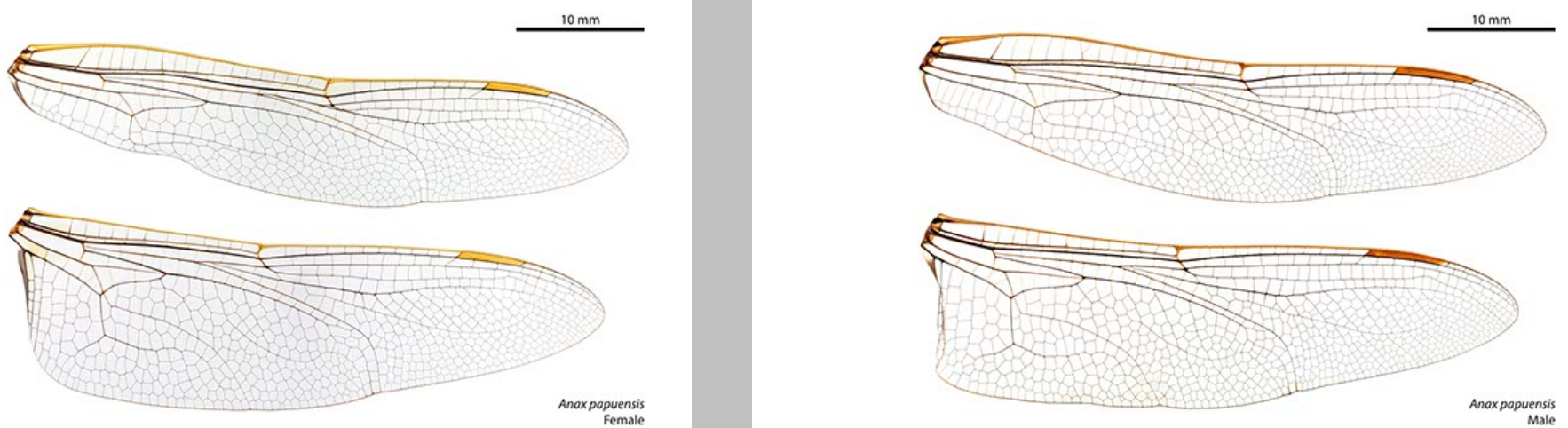

Anax papuensis male
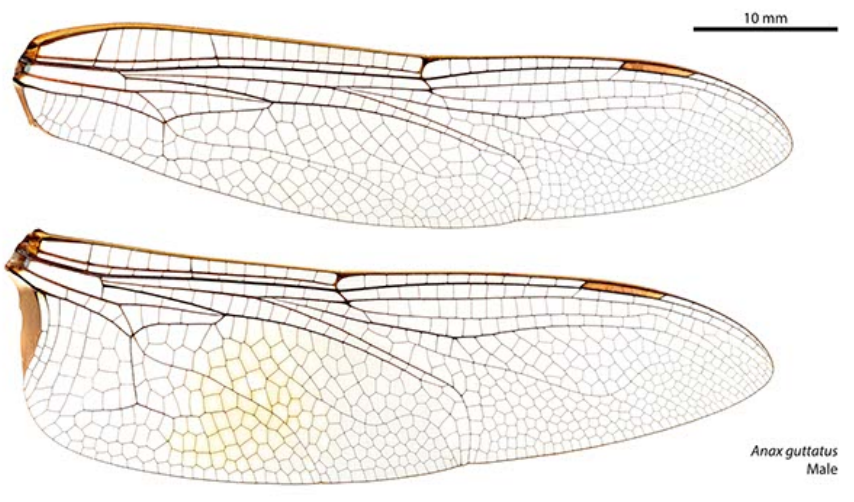

Anax guttatus male 

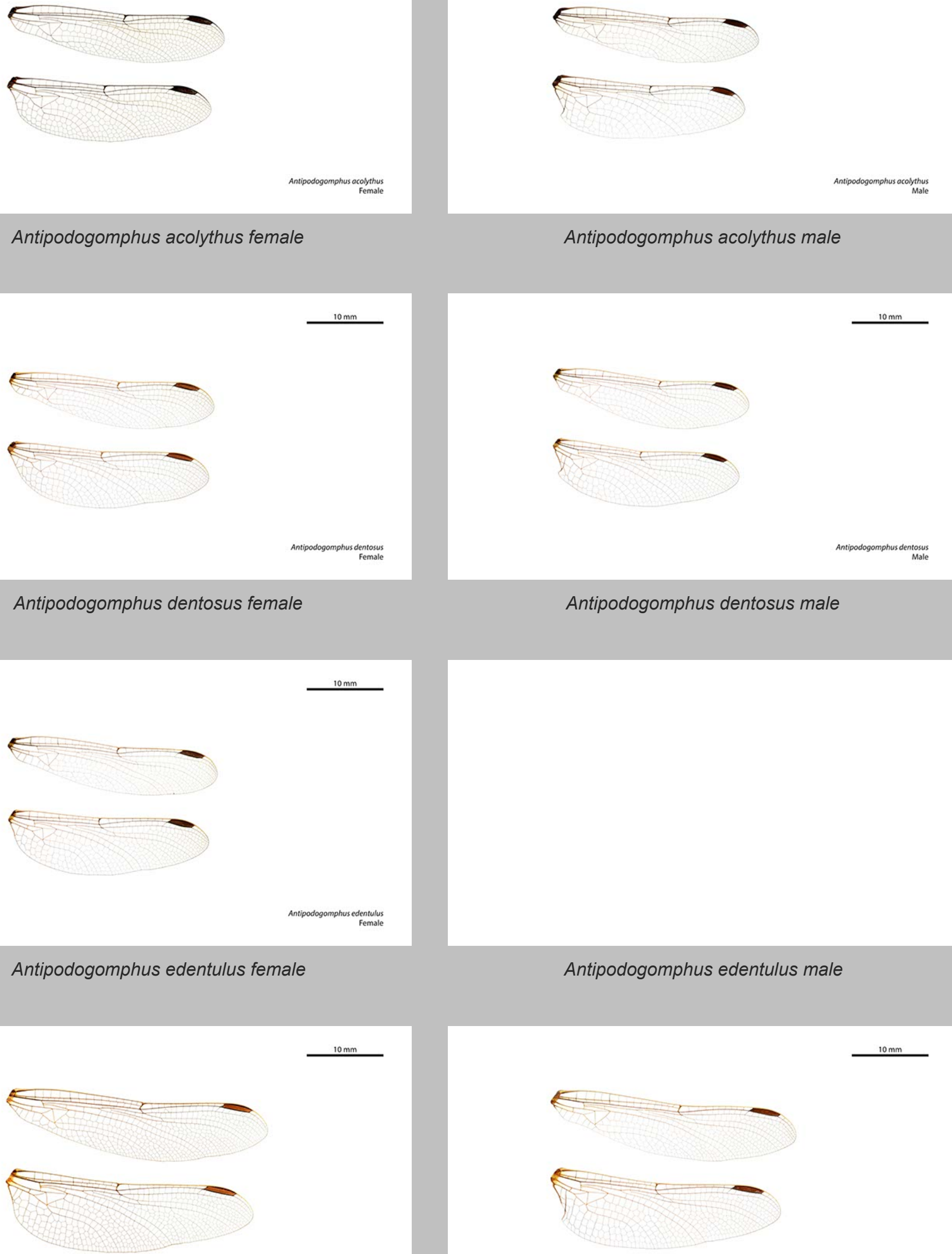

Antipodogomphus dentosus male 

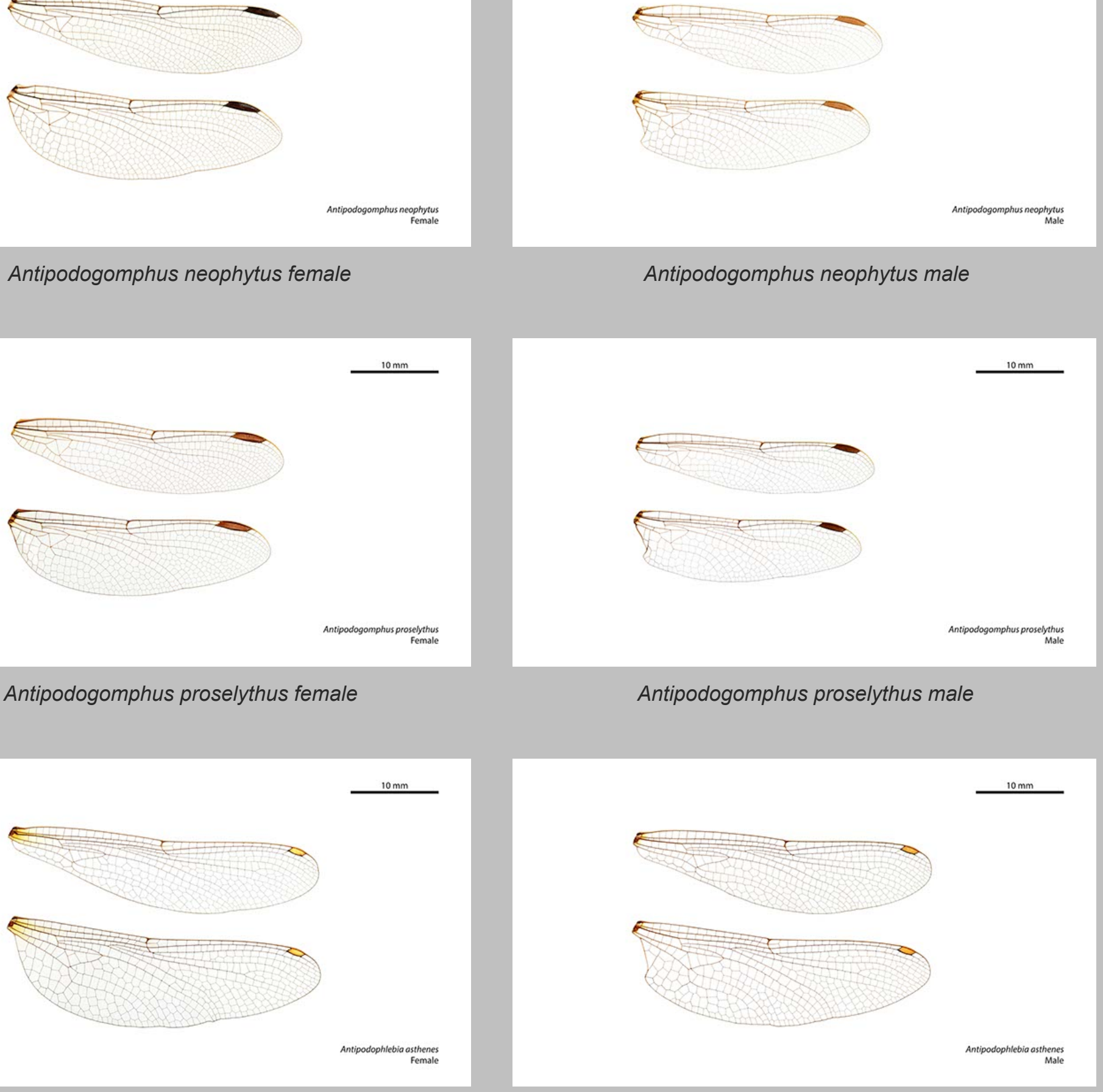

Antipodophlebia asthenes female

Antipodophlebia asthenes male
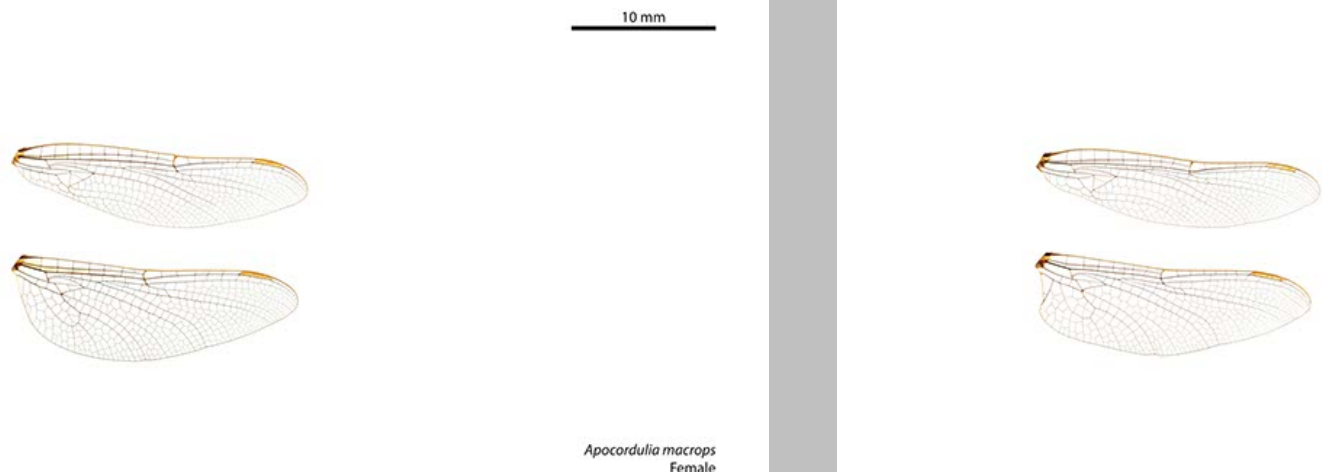


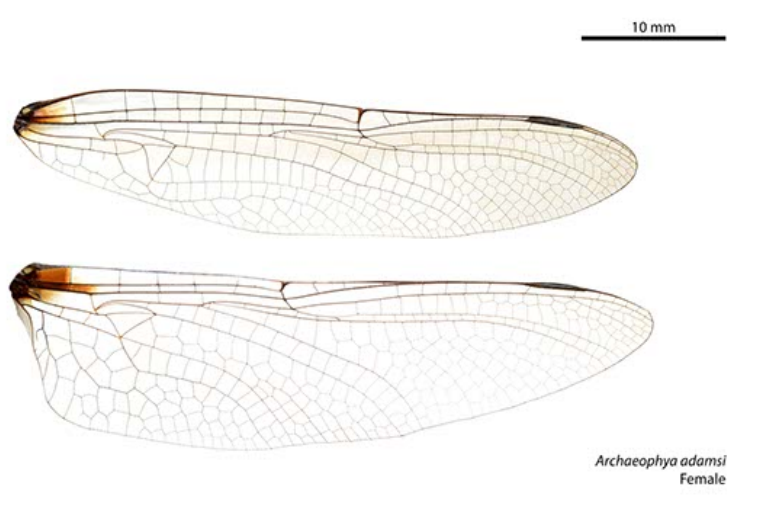

Archaeophya adamsi female

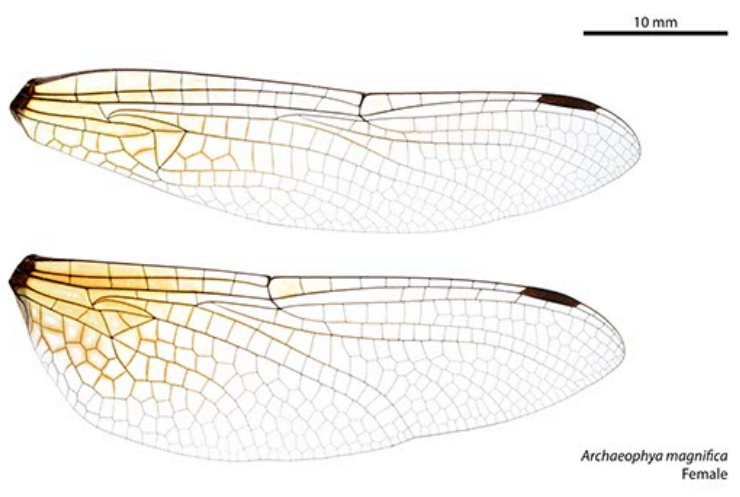

Archaeophya magnifica female

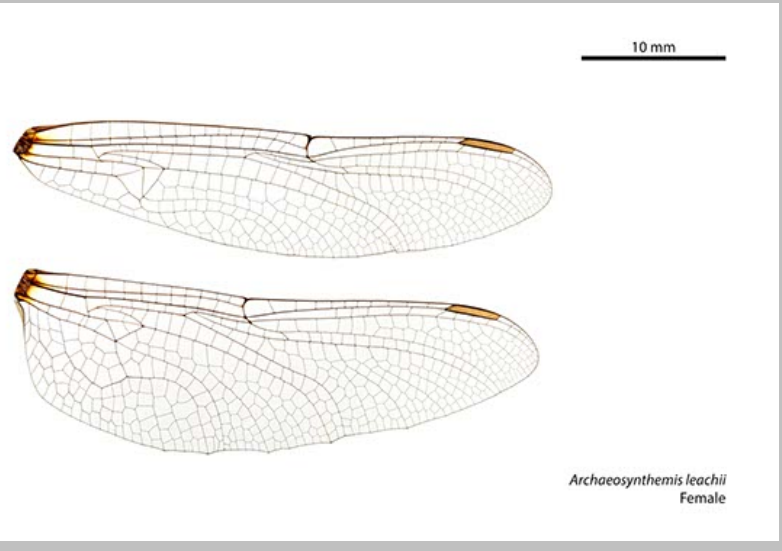

Archaeosynthemis leachii female

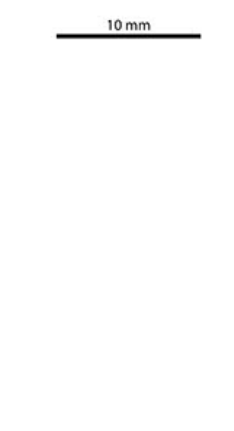

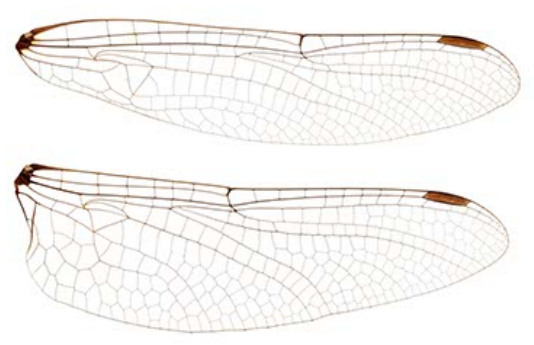

Archaeophya adamsi male
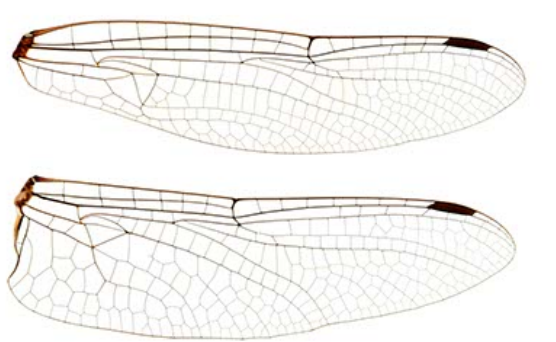

Archaeophya magnifica
Male

Archaeophya magnifica male

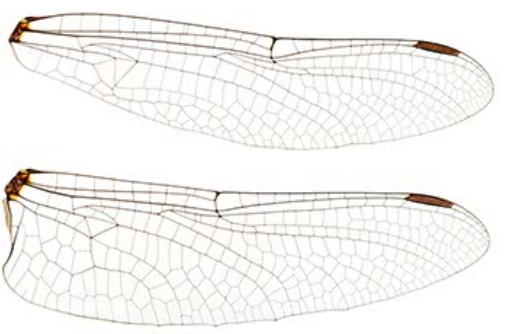

Archoeosynthemis leachii
Male

Archaeosynthemis leachii male

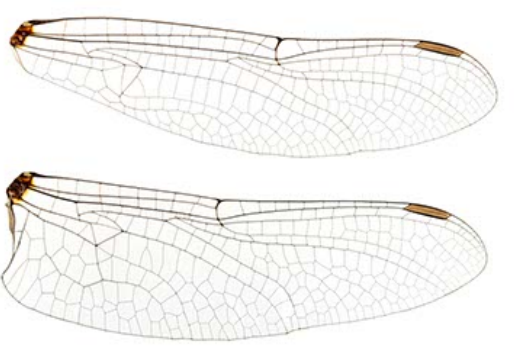

Archaeosynthemis occidentalis 

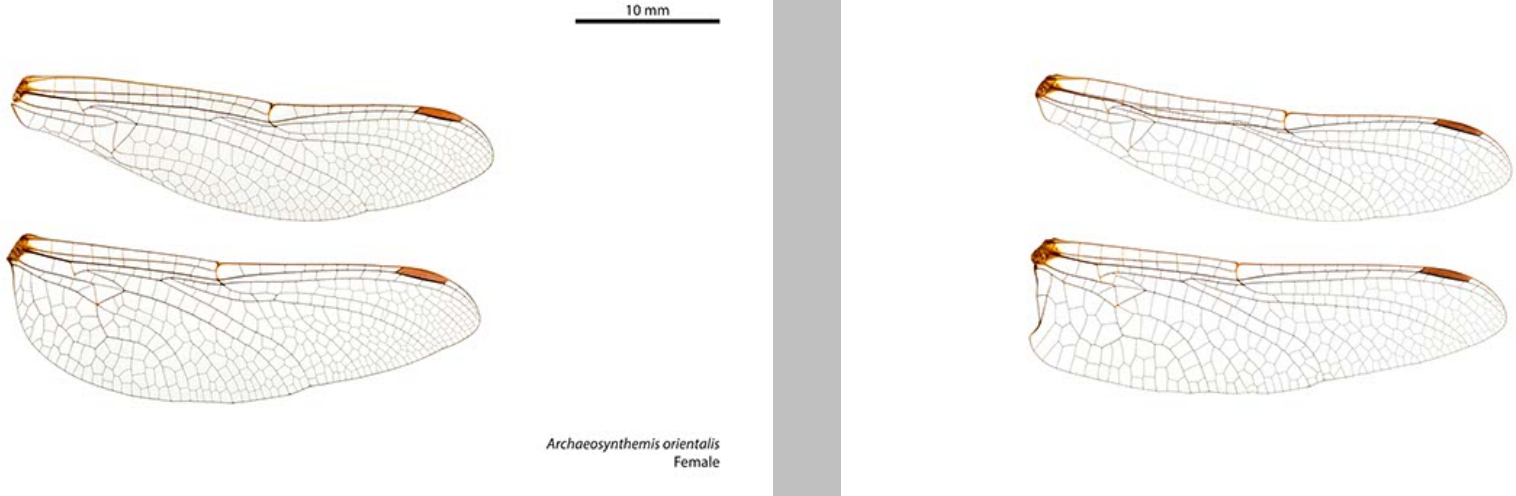

Archaeosynthemis orientalis female

Archaeosynthemis orientalis male
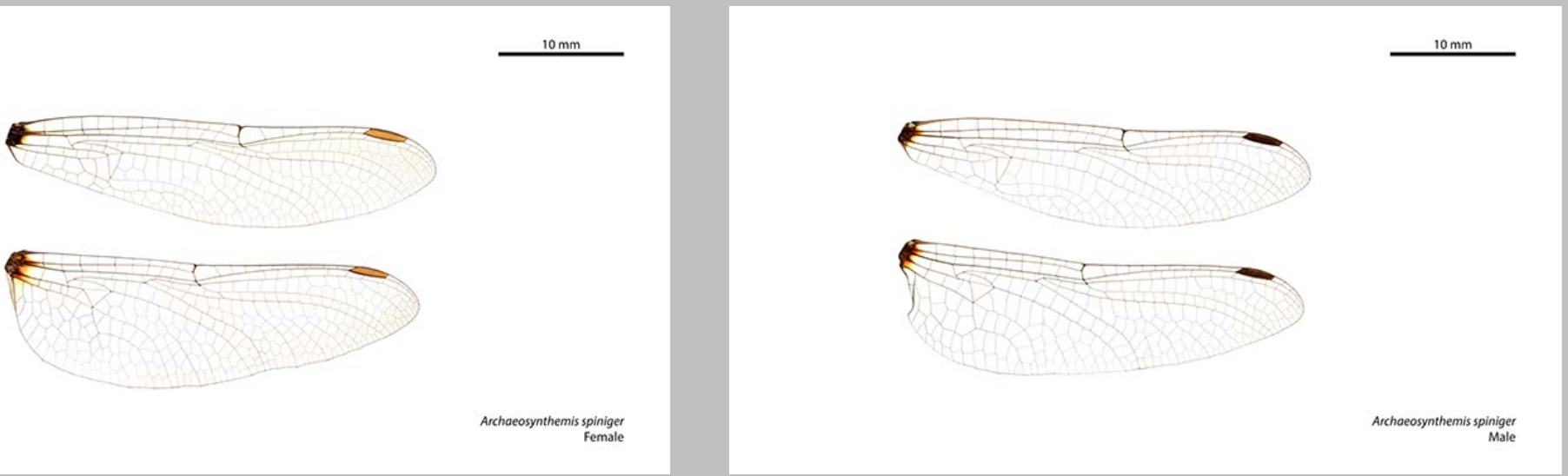

Archaeosynthemis spiniger female

Archaeosynthemis spiniger male

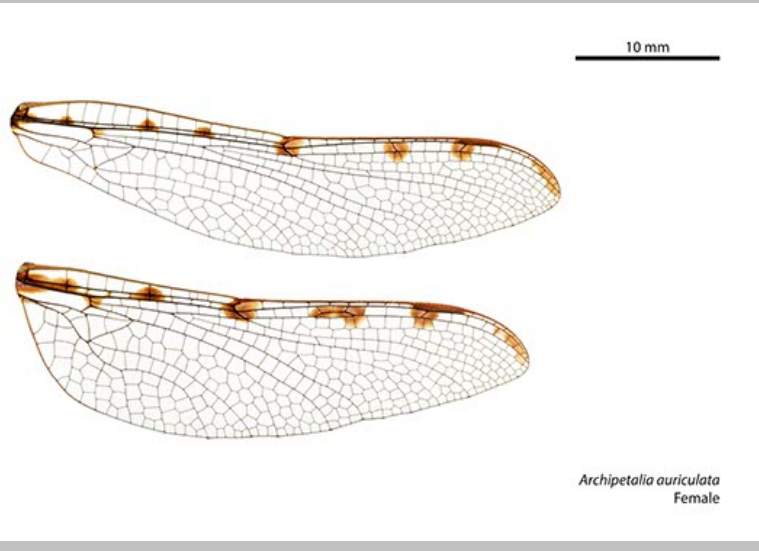

Archipetalia auriculata female
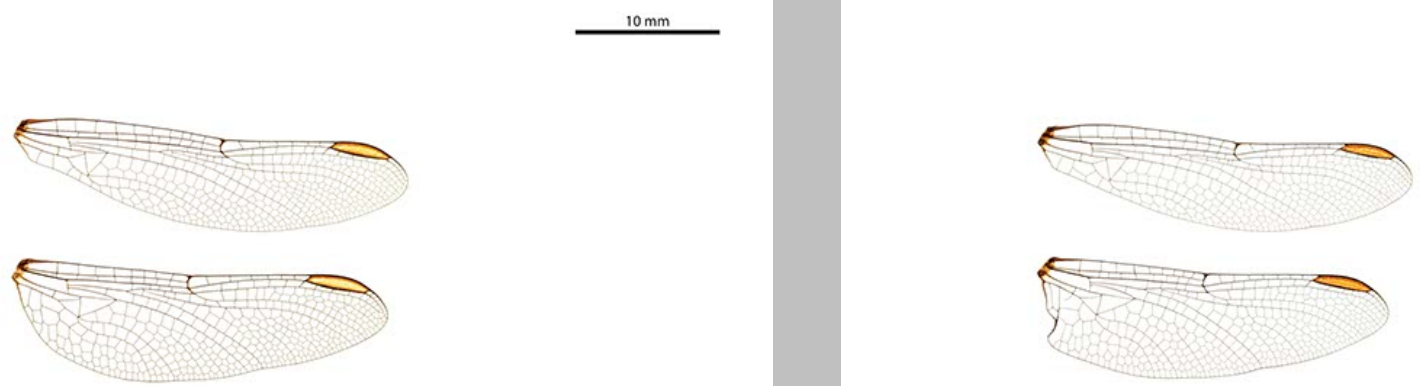

Archipetalia auriculata male 


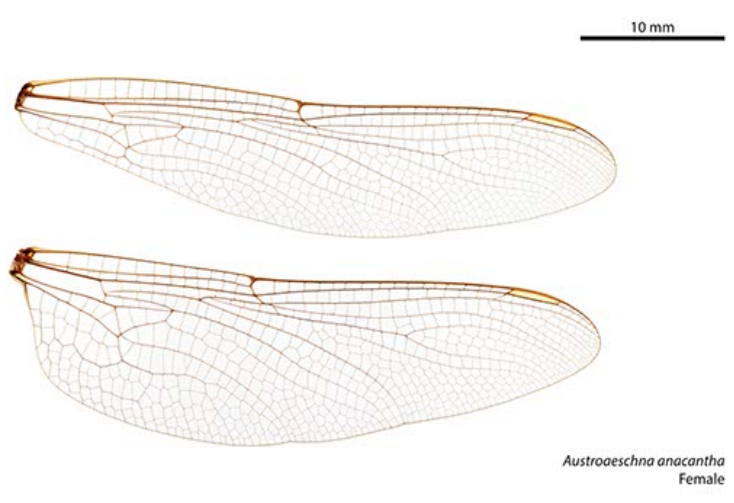

Austroaeschna anacantha female
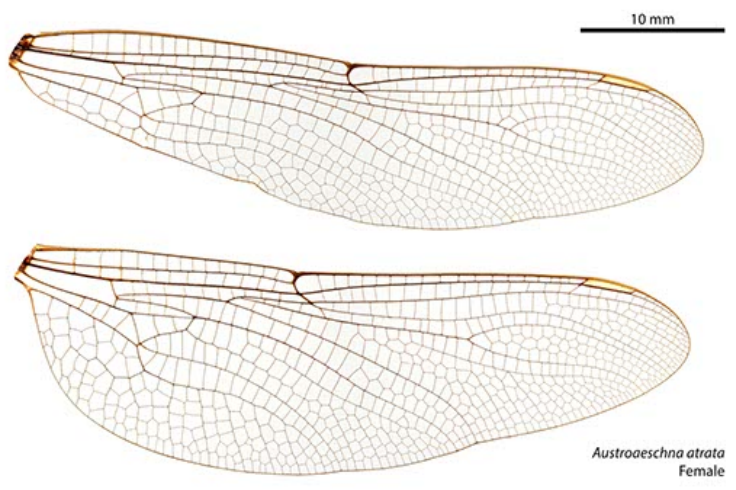

Austroaeschna atrata female

Austroaeschna christine female

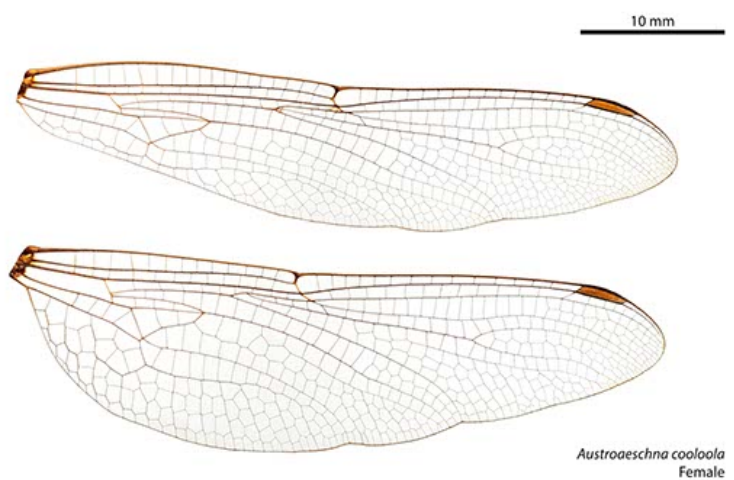

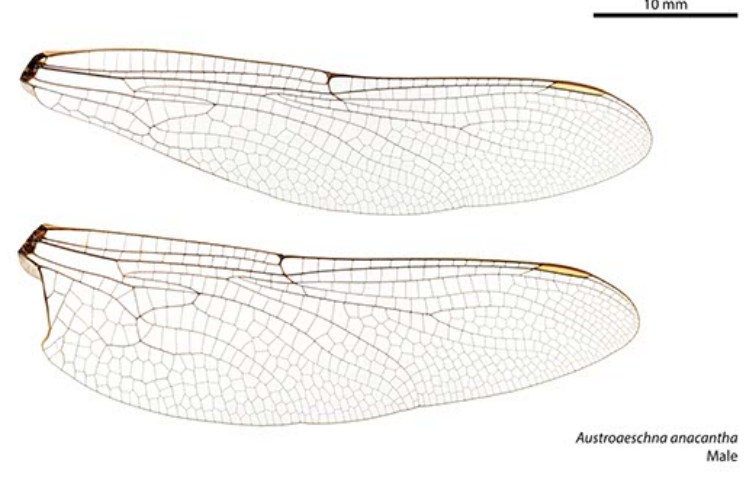

Austroaeschna anacantha male

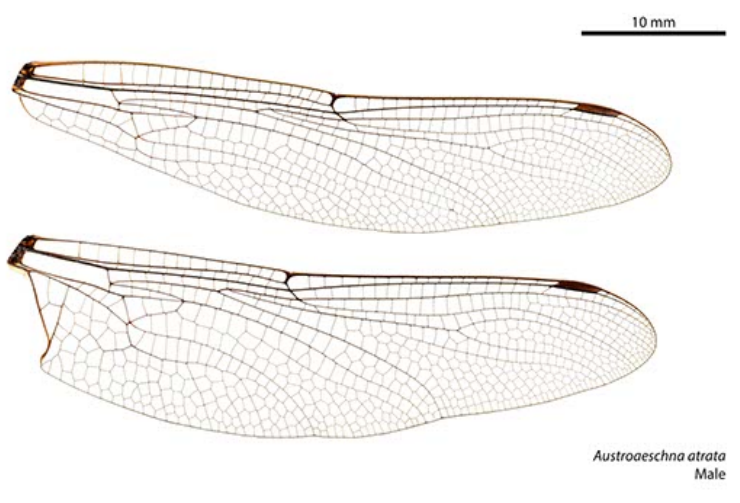

Austroaeschna atrata male

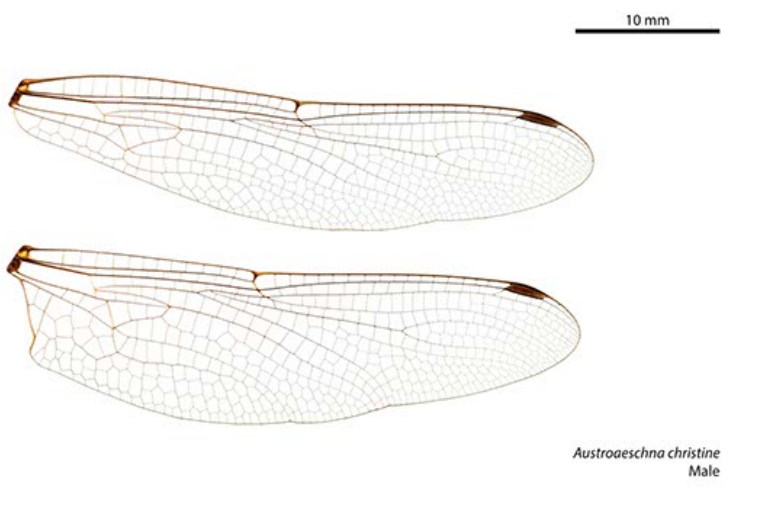

Austroaeschna christine male

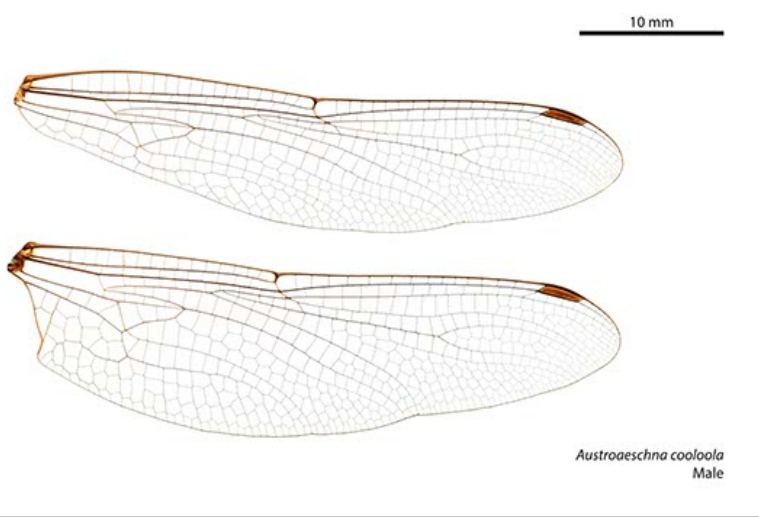

Austroaeschna cooloola male 


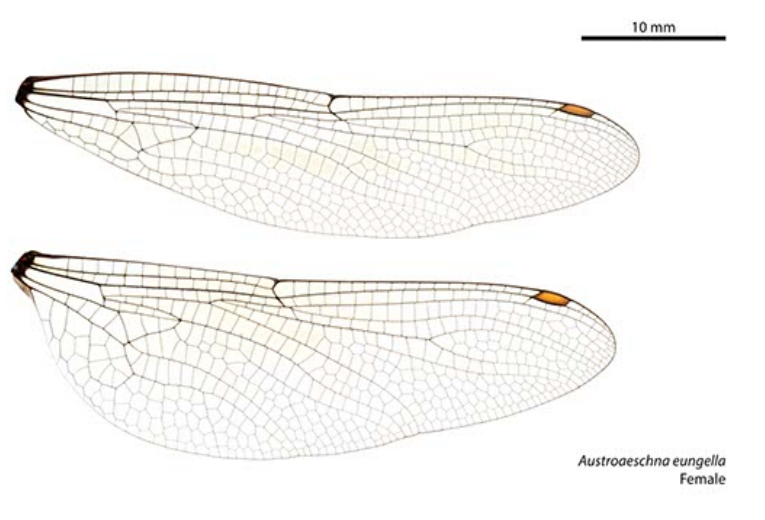

Austroaeschna eungella female

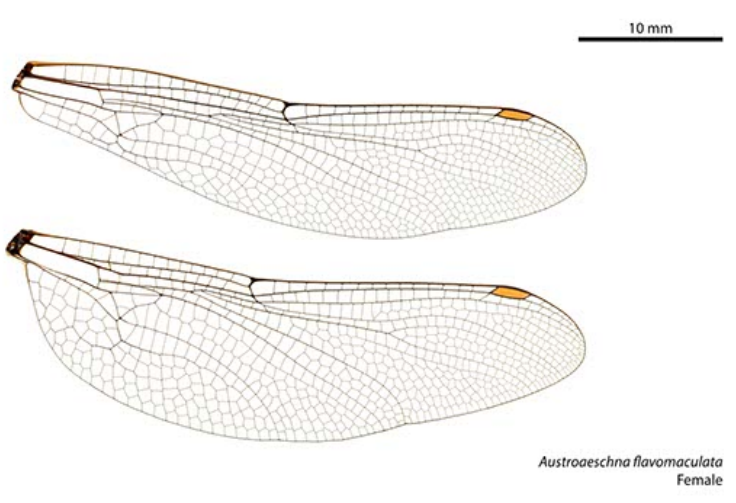

Austroaeschna flavomaculata female

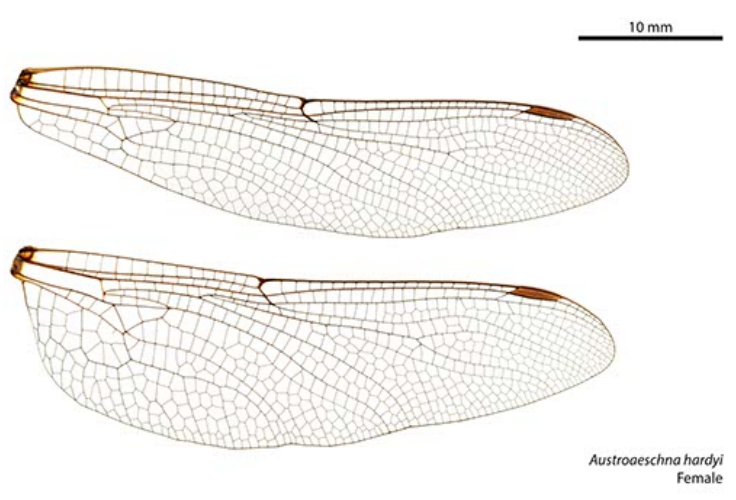

Austroaeschna hardyi female

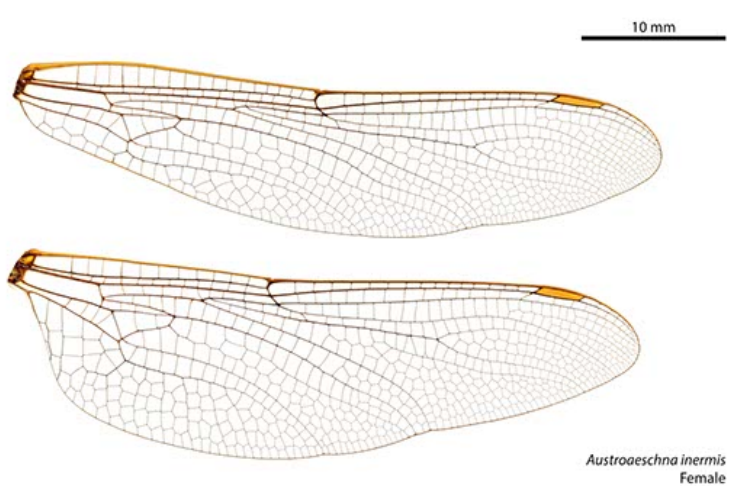

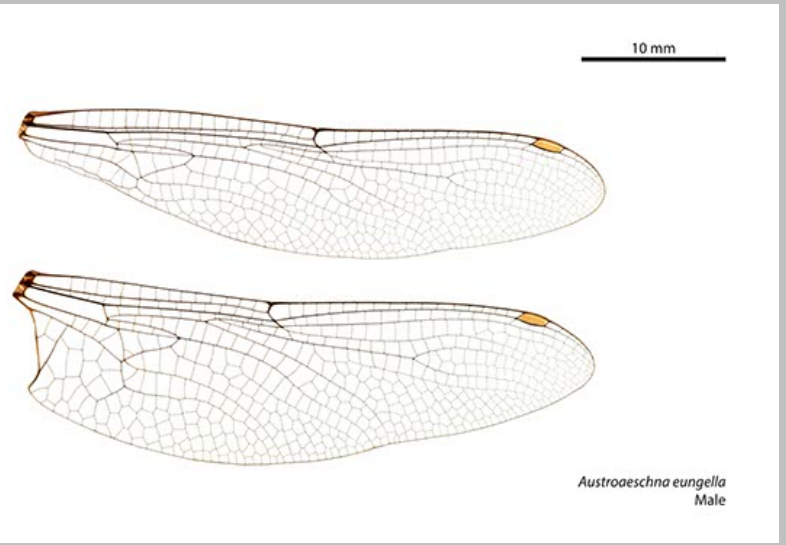

Austroaeschna eungella male

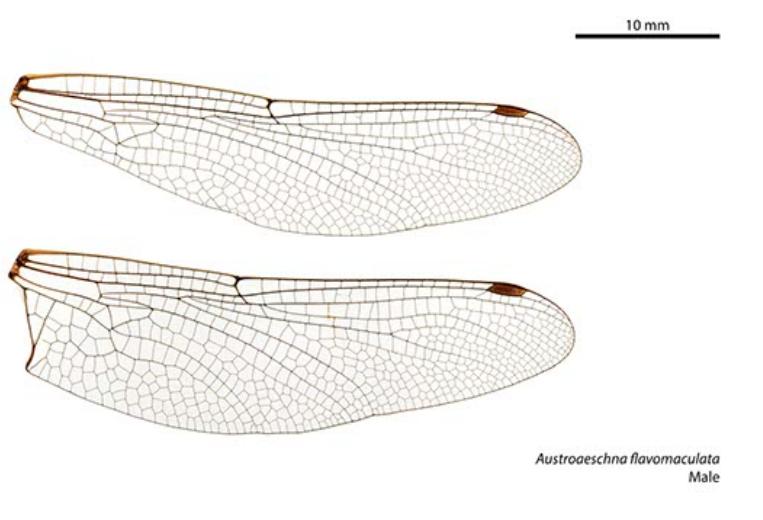

Austroaeschna flavomaculata male

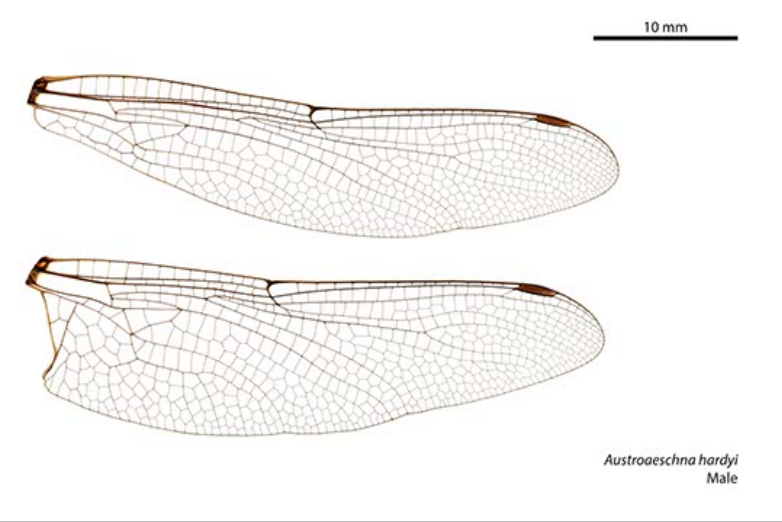

Austroaeschna hardyi male

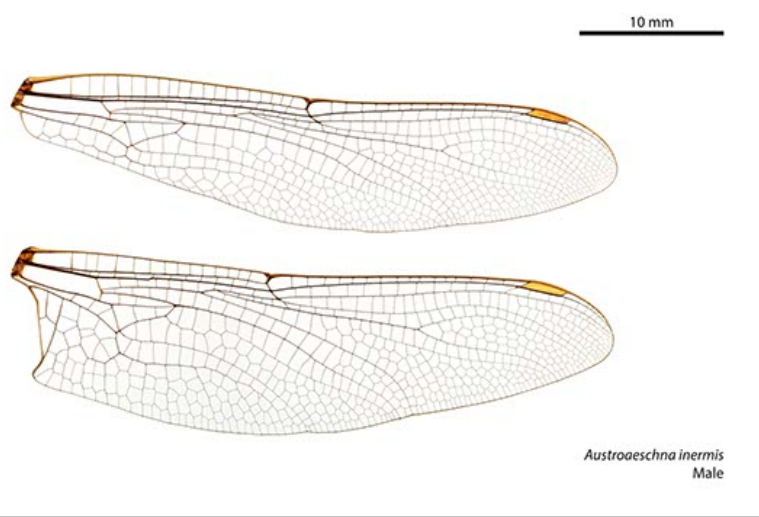

Austroaeschna inermis male 


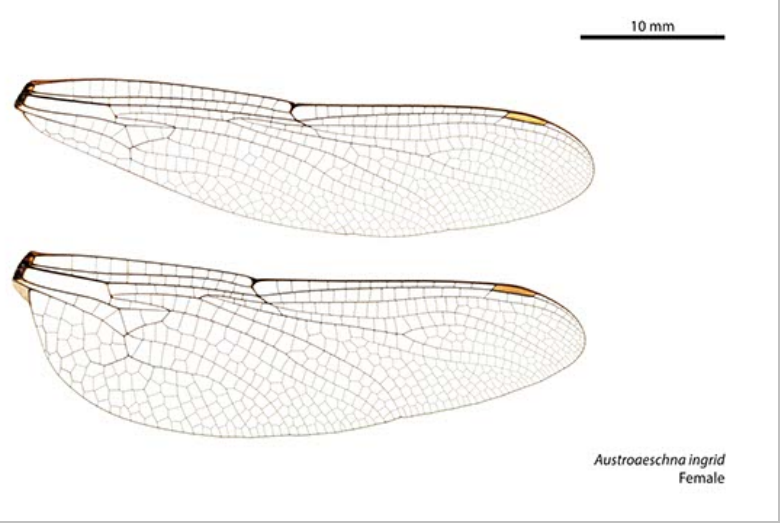

Austroaeschna ingrid female

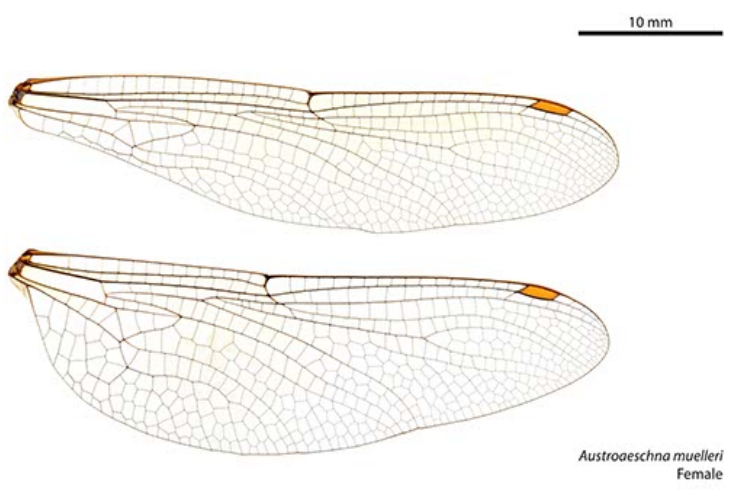

Austroaeschna muelleri female

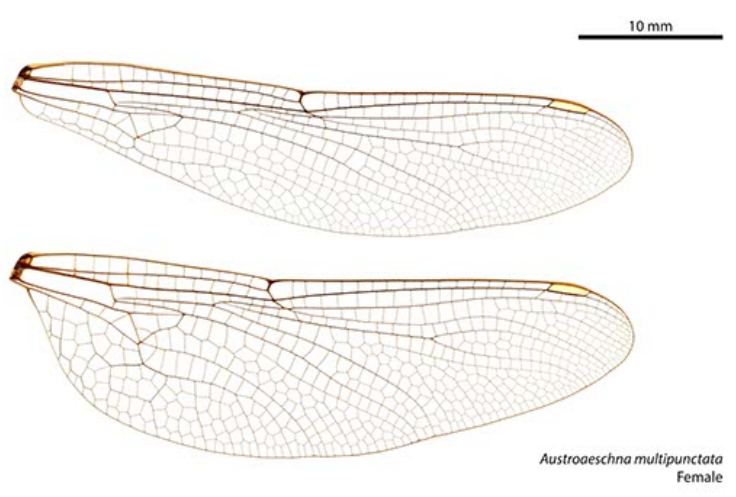

Austroaeschna multipunctata female

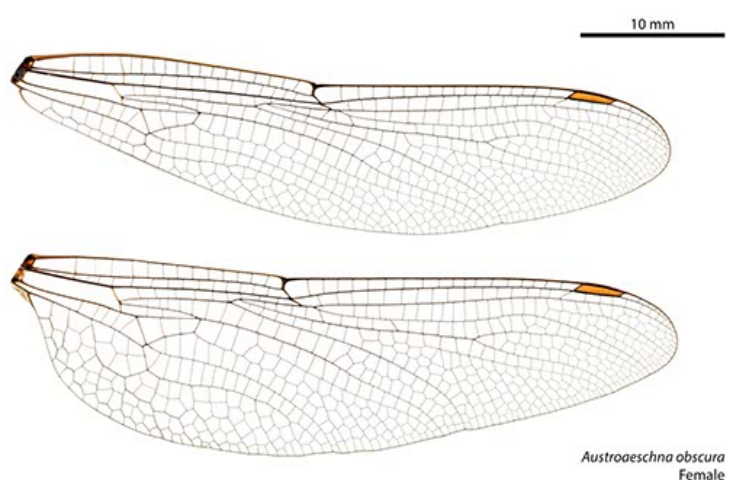

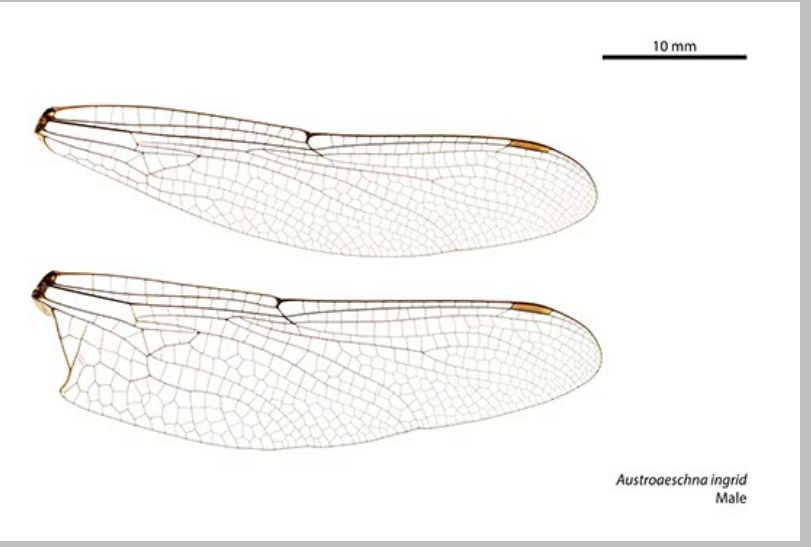

Austroaeschna ingrid male

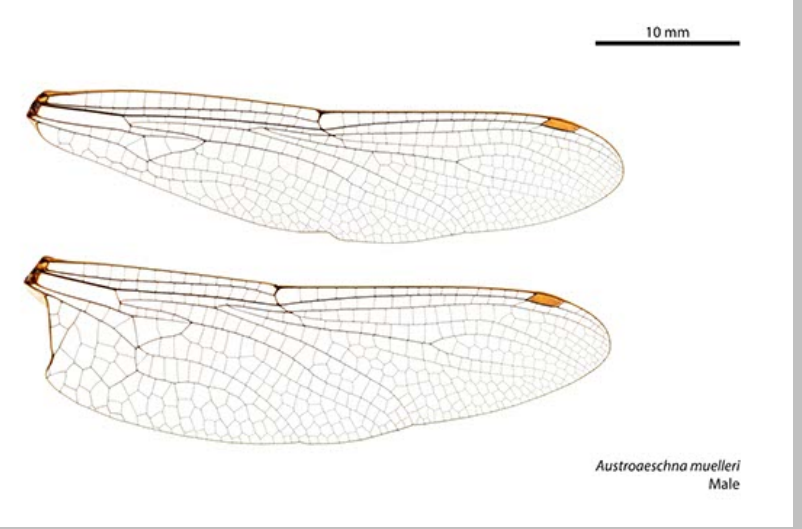

Austroaeschna muelleri male

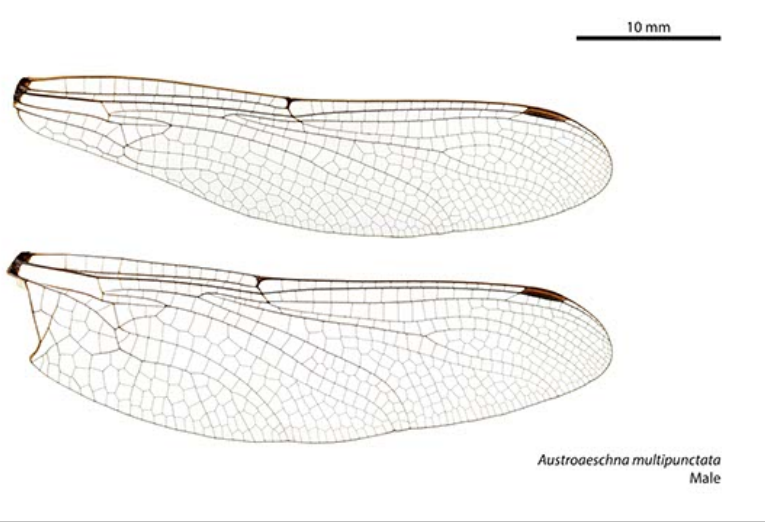

Austroaeschna multipunctata male

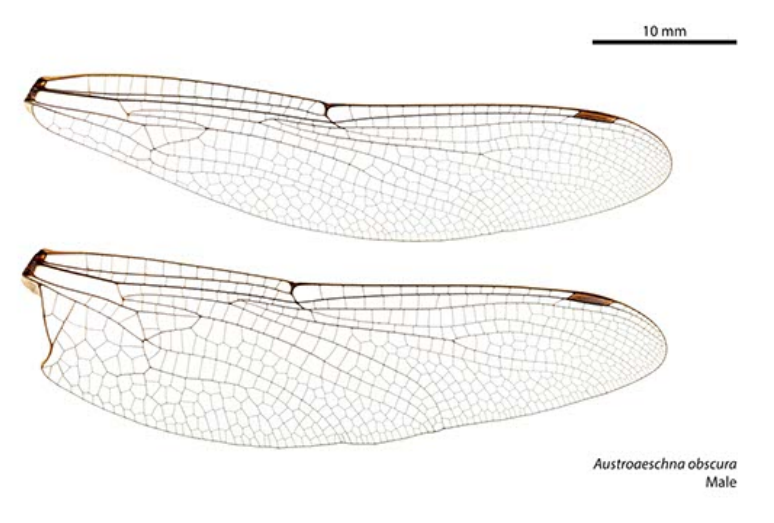

Austroaeschna obscura male 


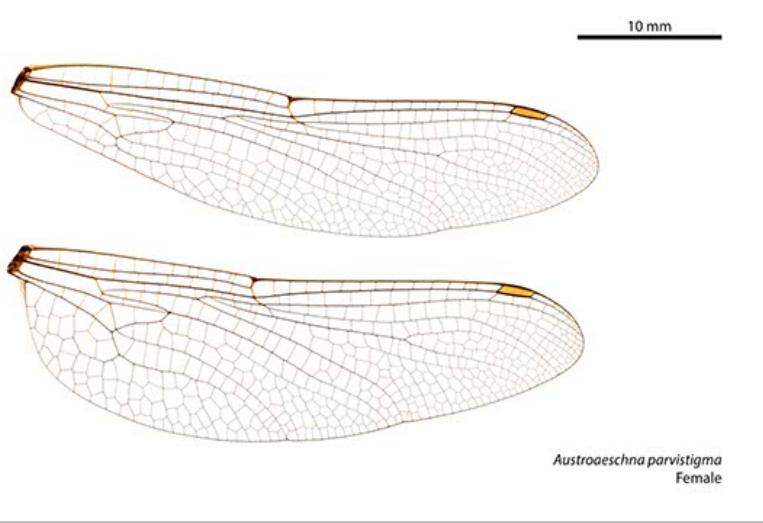

Austroaeschna parvistigma female

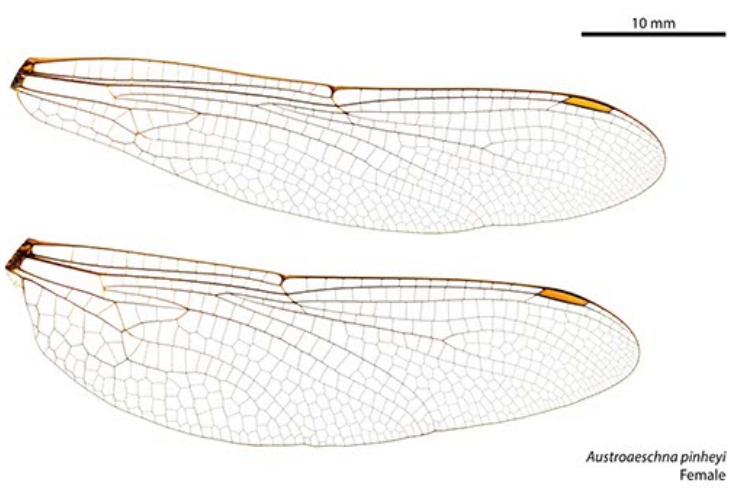

Austroaeschna pinheyi female

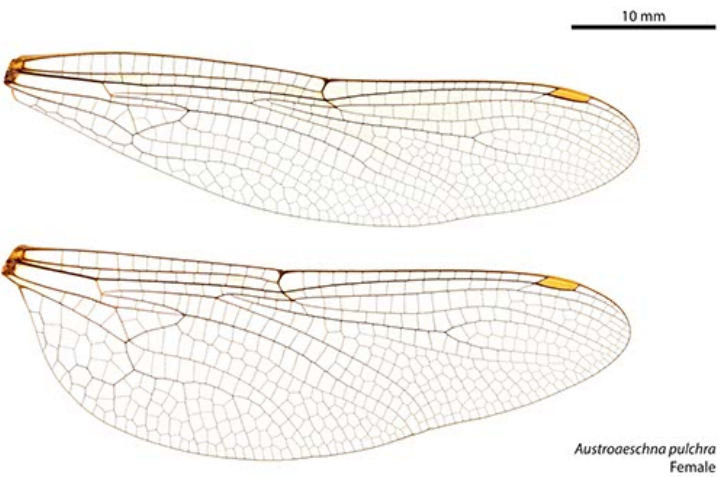

Austroaeschna pulchra female

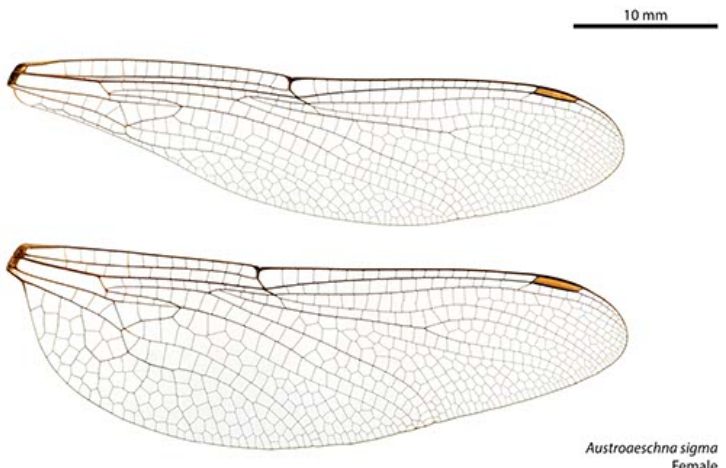

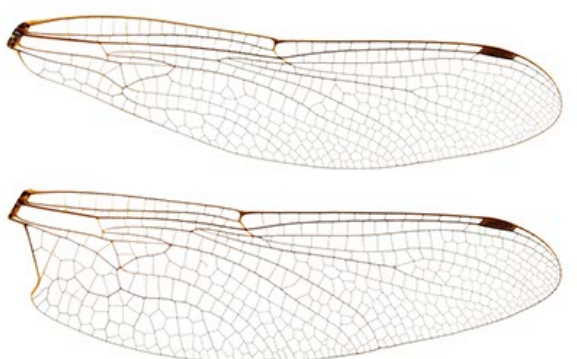

Austroaeschna parvistigma male

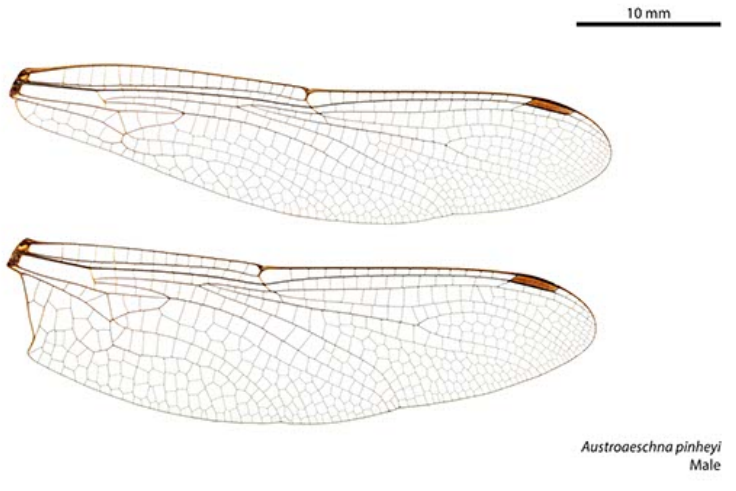

Austroaeschna pinheyi male

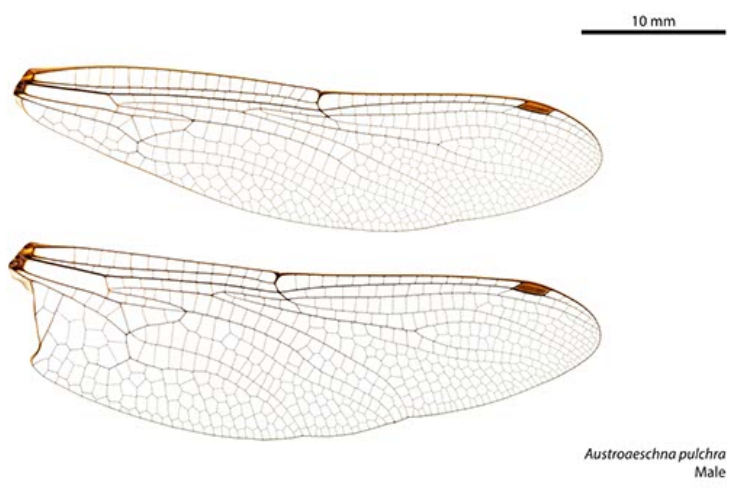

Austroaeschna pulchra male

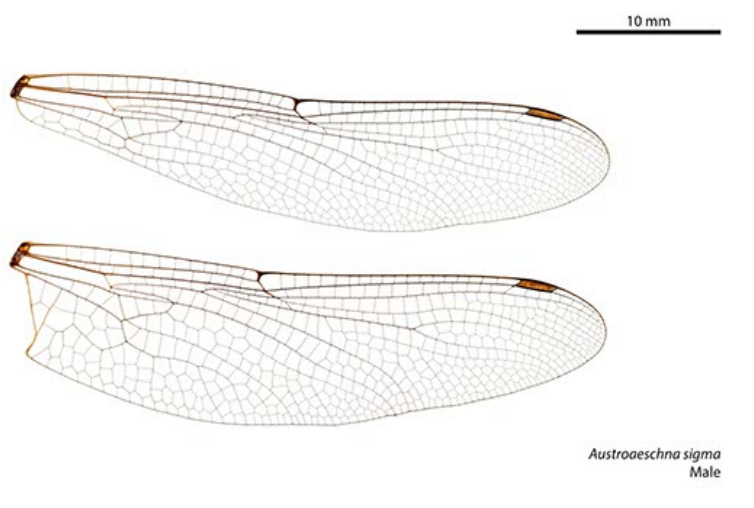

Austroaeschna sigma male 


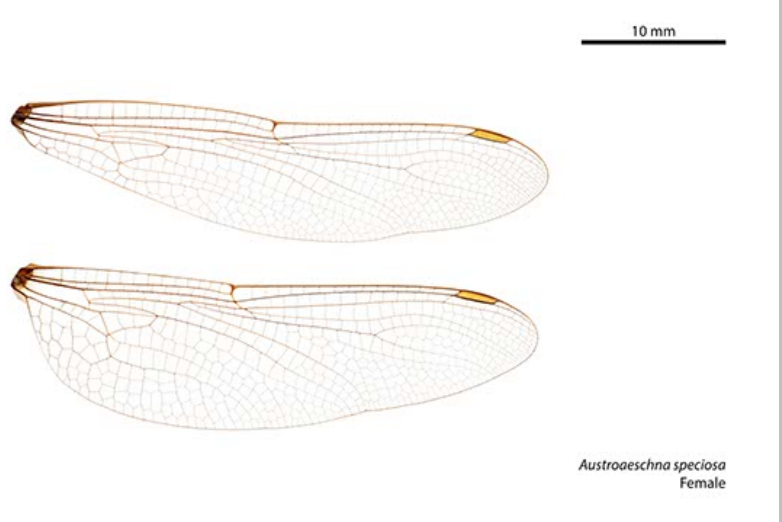

Austroaeschna speciosa female

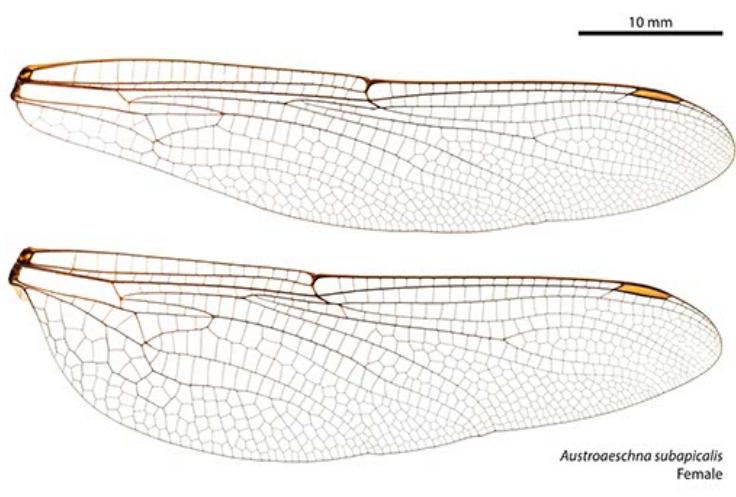

Austroaeschna subapicalis female

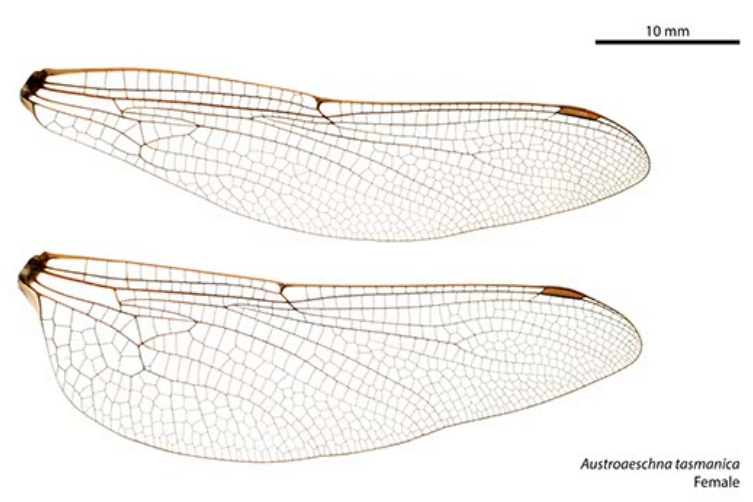

Austroaeschna tasmanica female
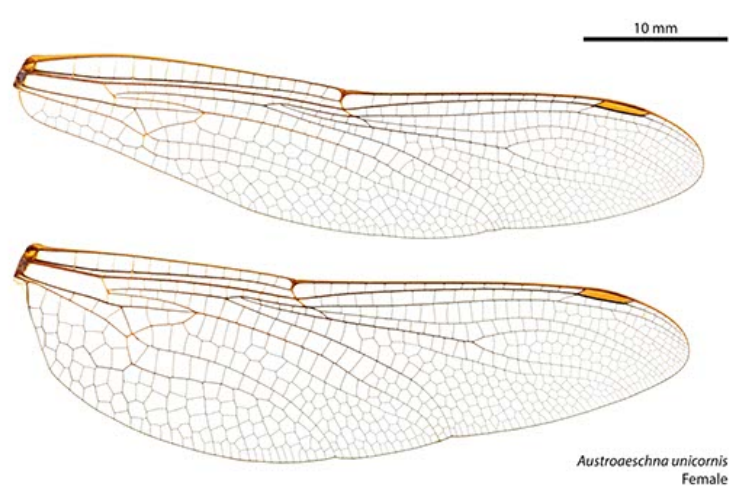

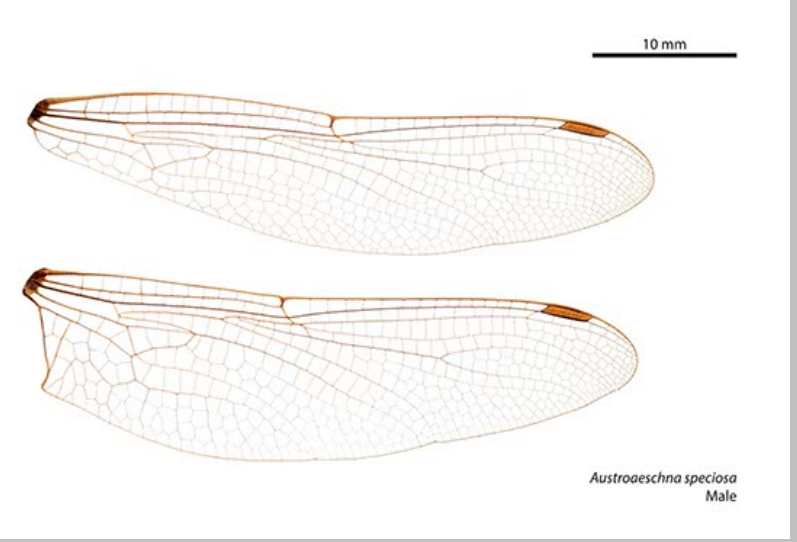

Austroaeschna speciosa male

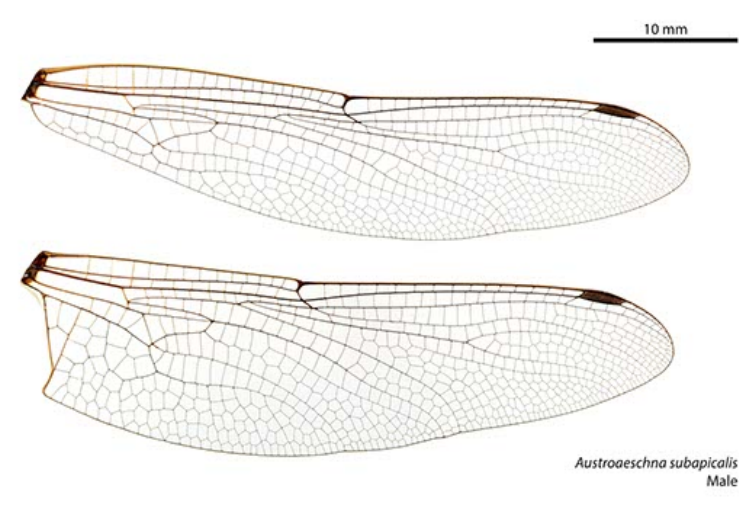

Austroaeschna subapicalis male

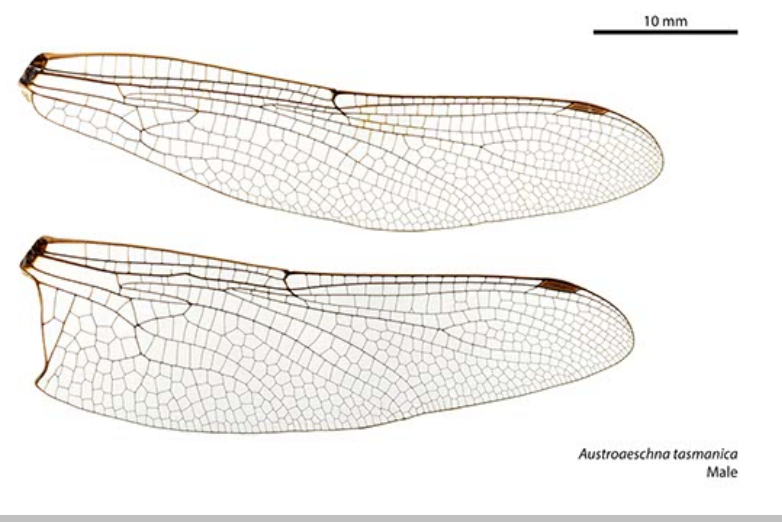

Austroaeschna tasmanica male

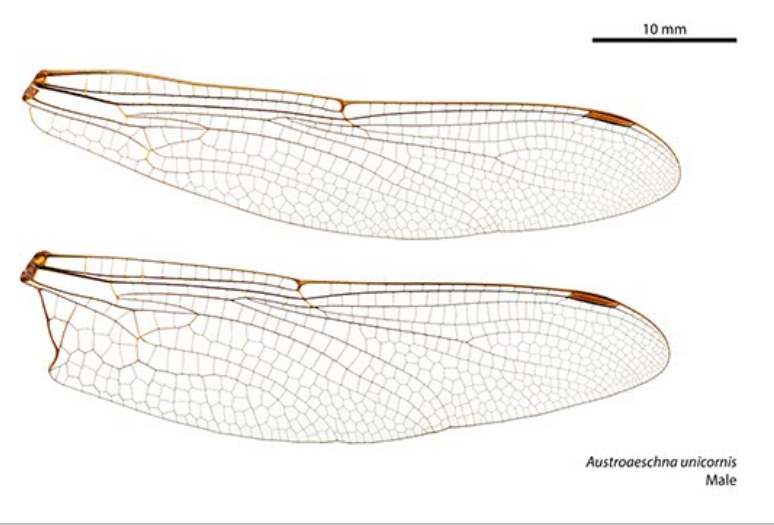

Austroaeschna unicornis male 

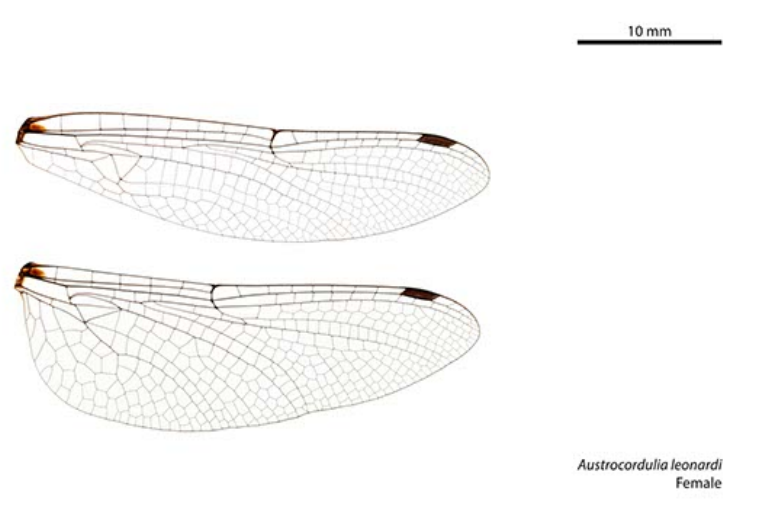

\section{Austrocordulia leonardi female}

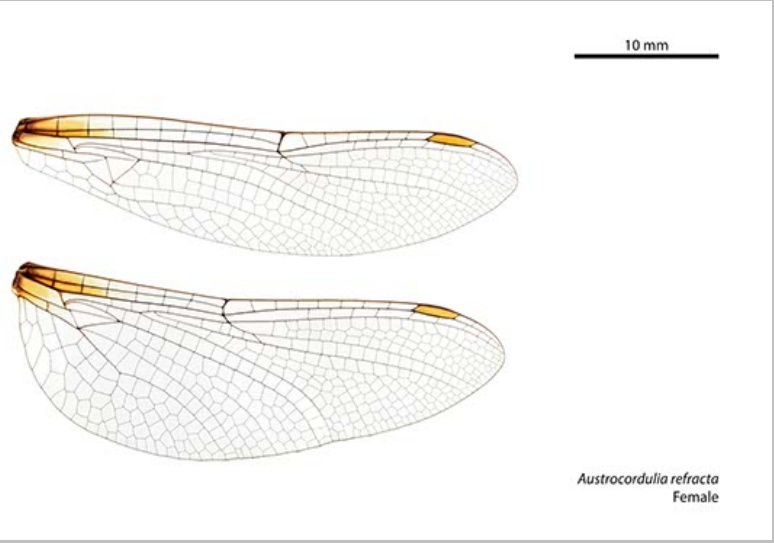

Austrocordulia refracta female
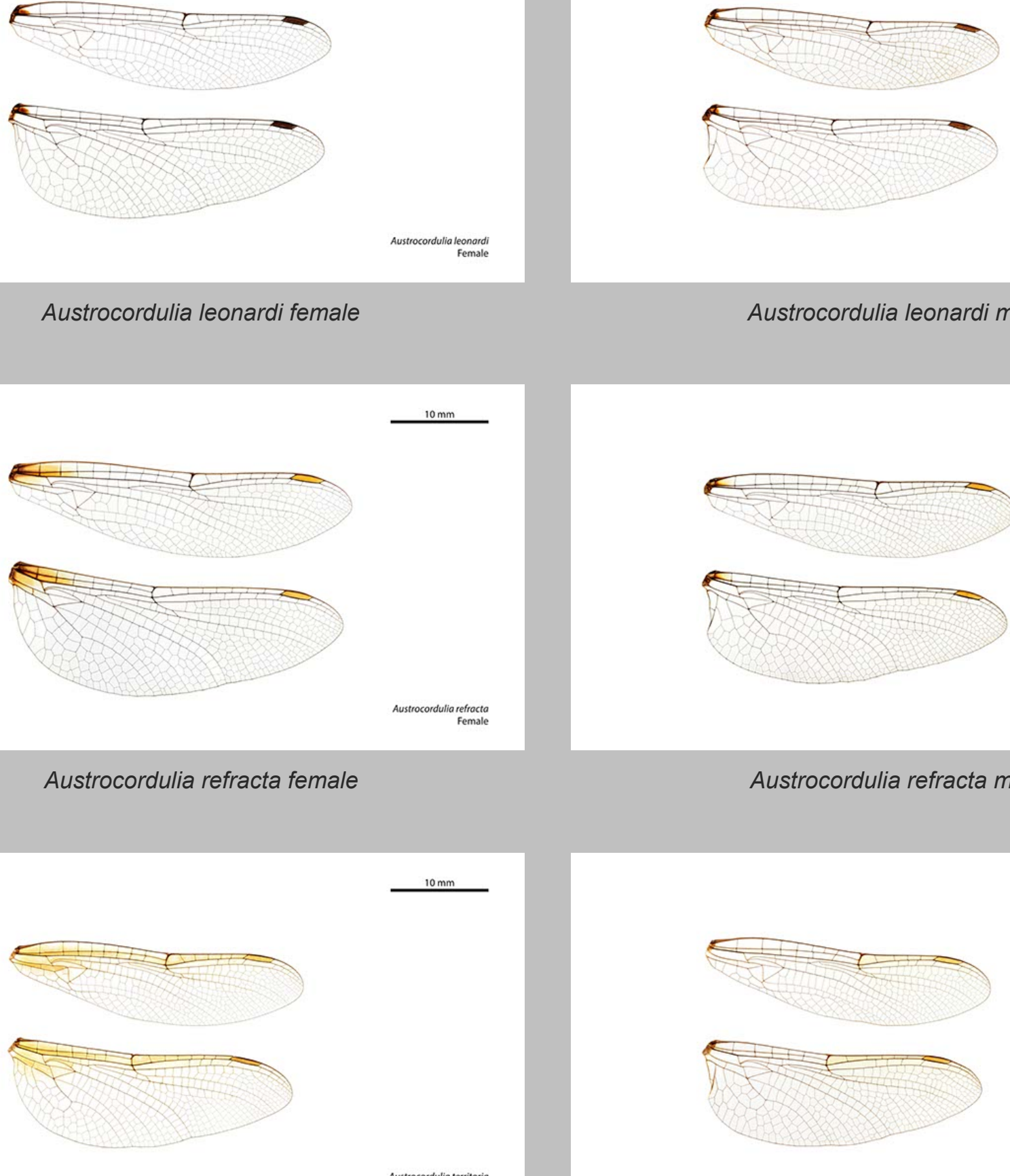

Austrocordulia leonardi male

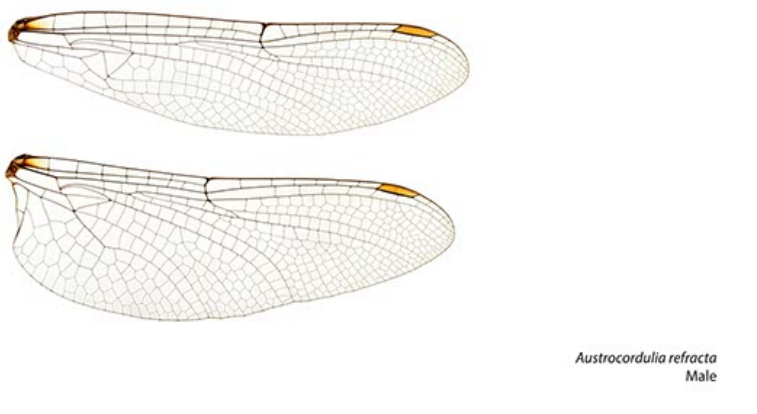

Austrocordulia refracta male
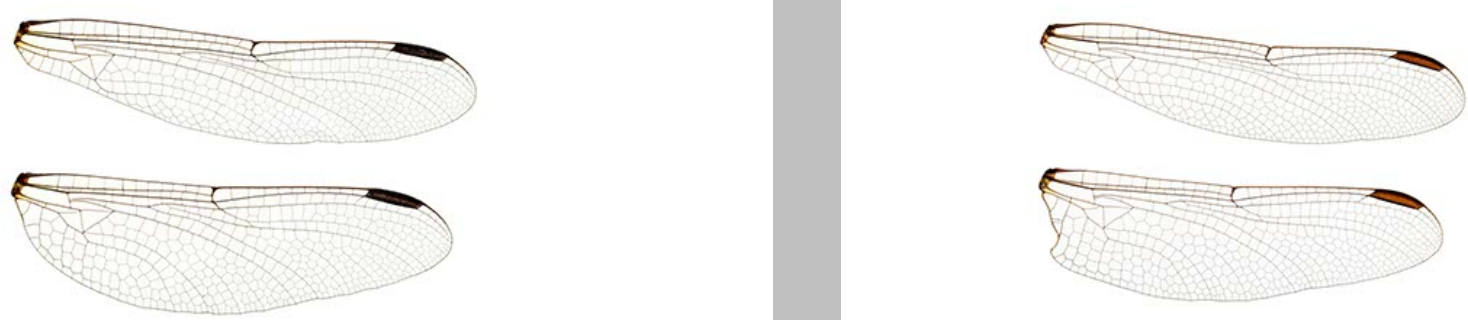

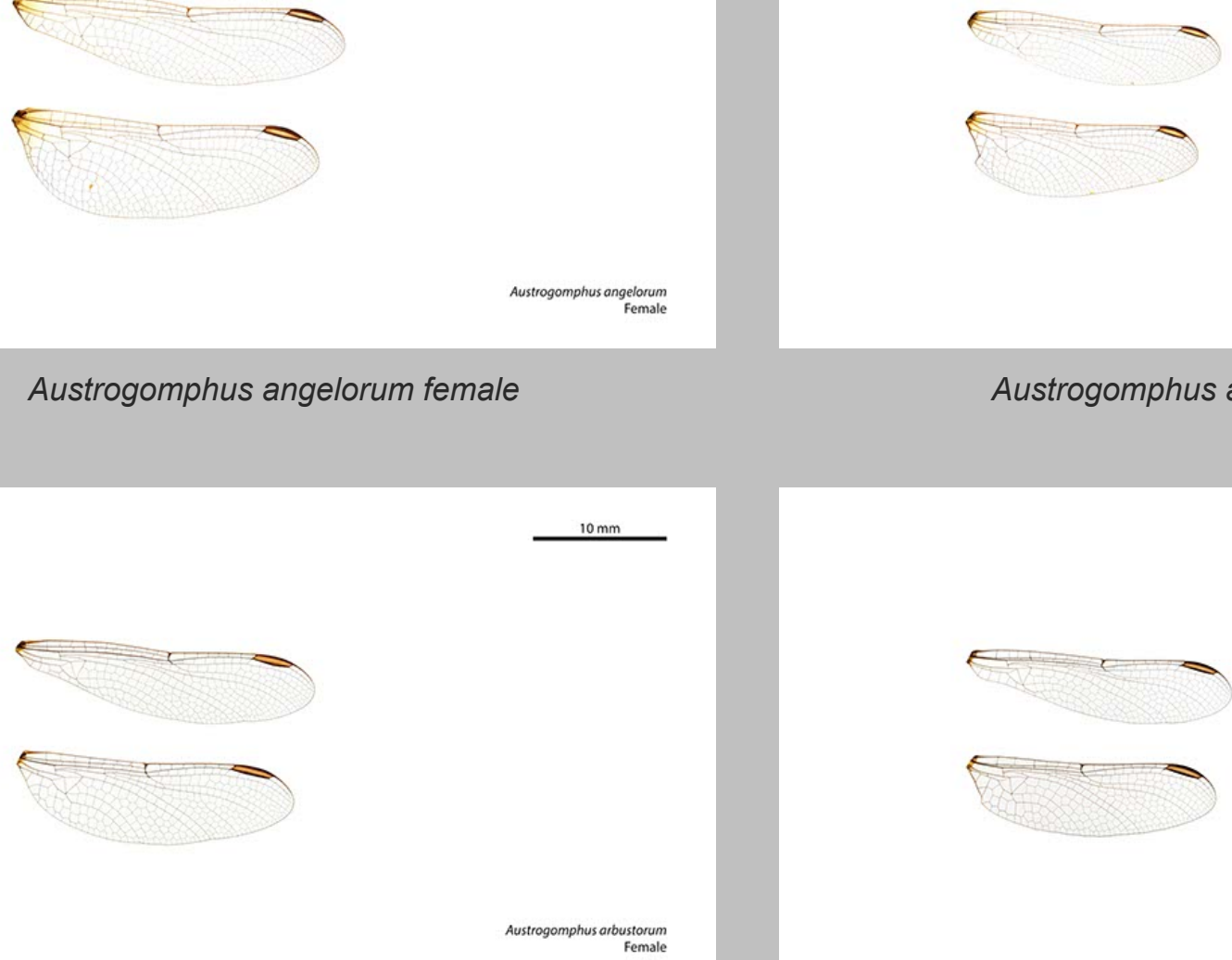

\section{Austrogomphus arbustorum male}
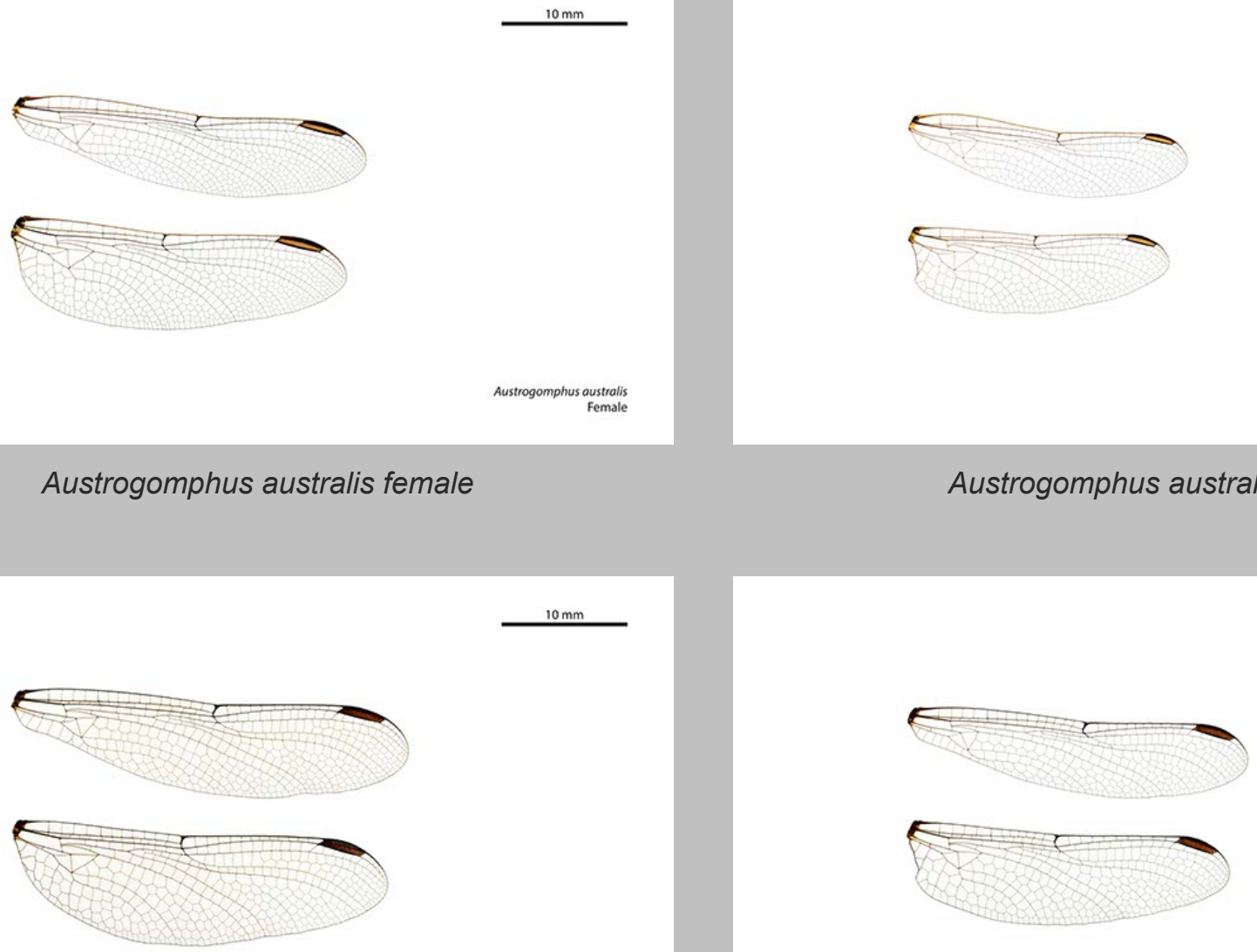

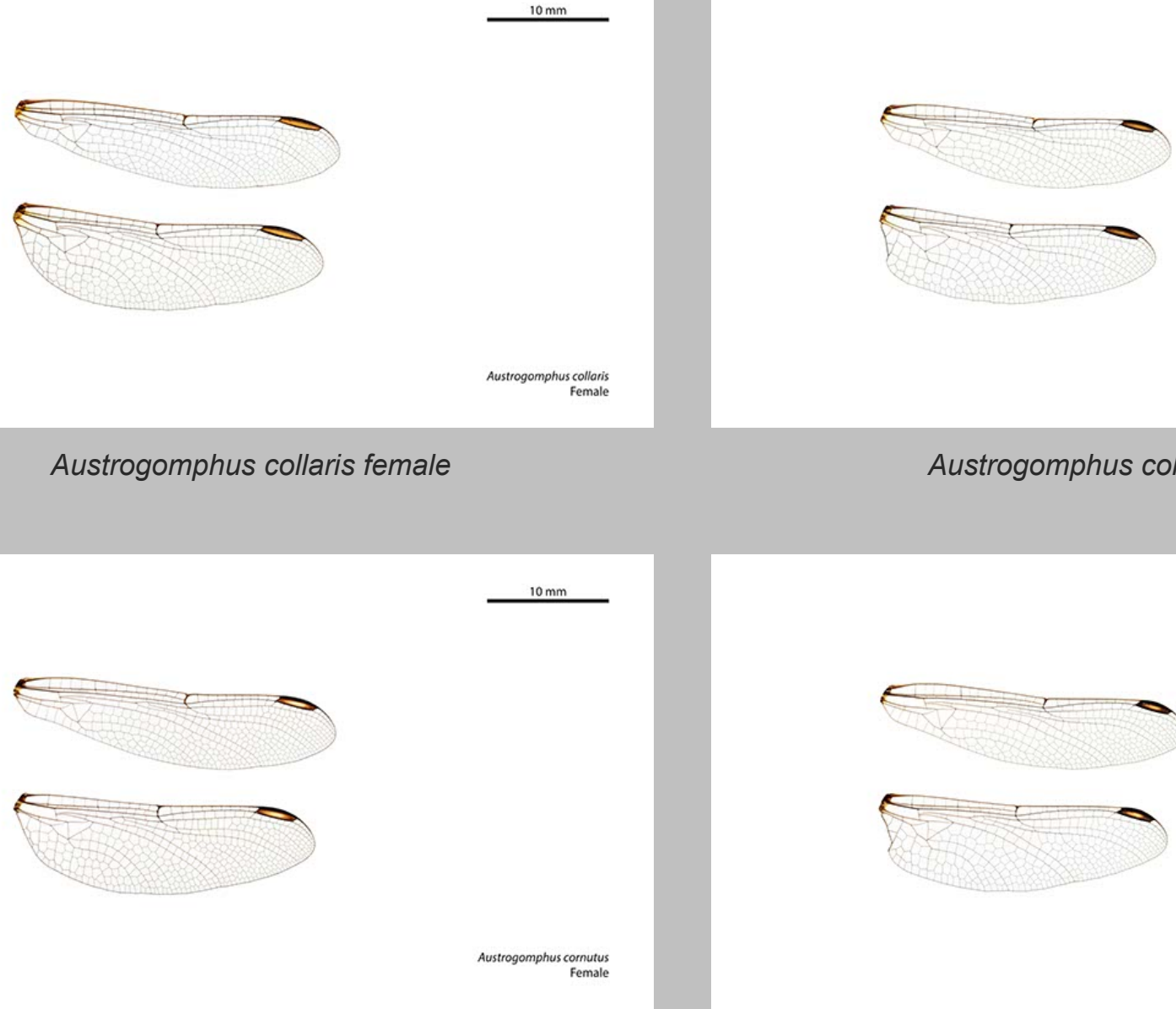

Austrogomphus cornutus female
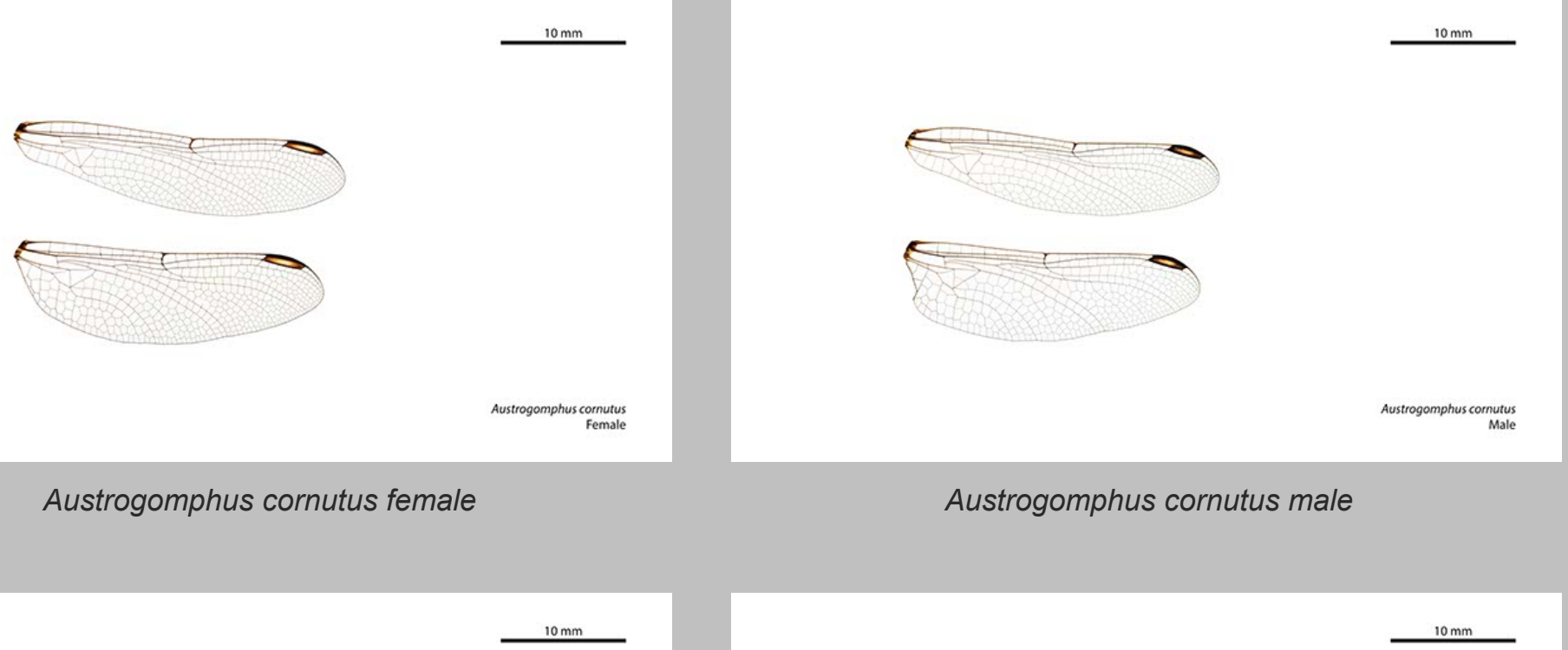

Austrogomphus collaris male

Austrogomphus cornutus male
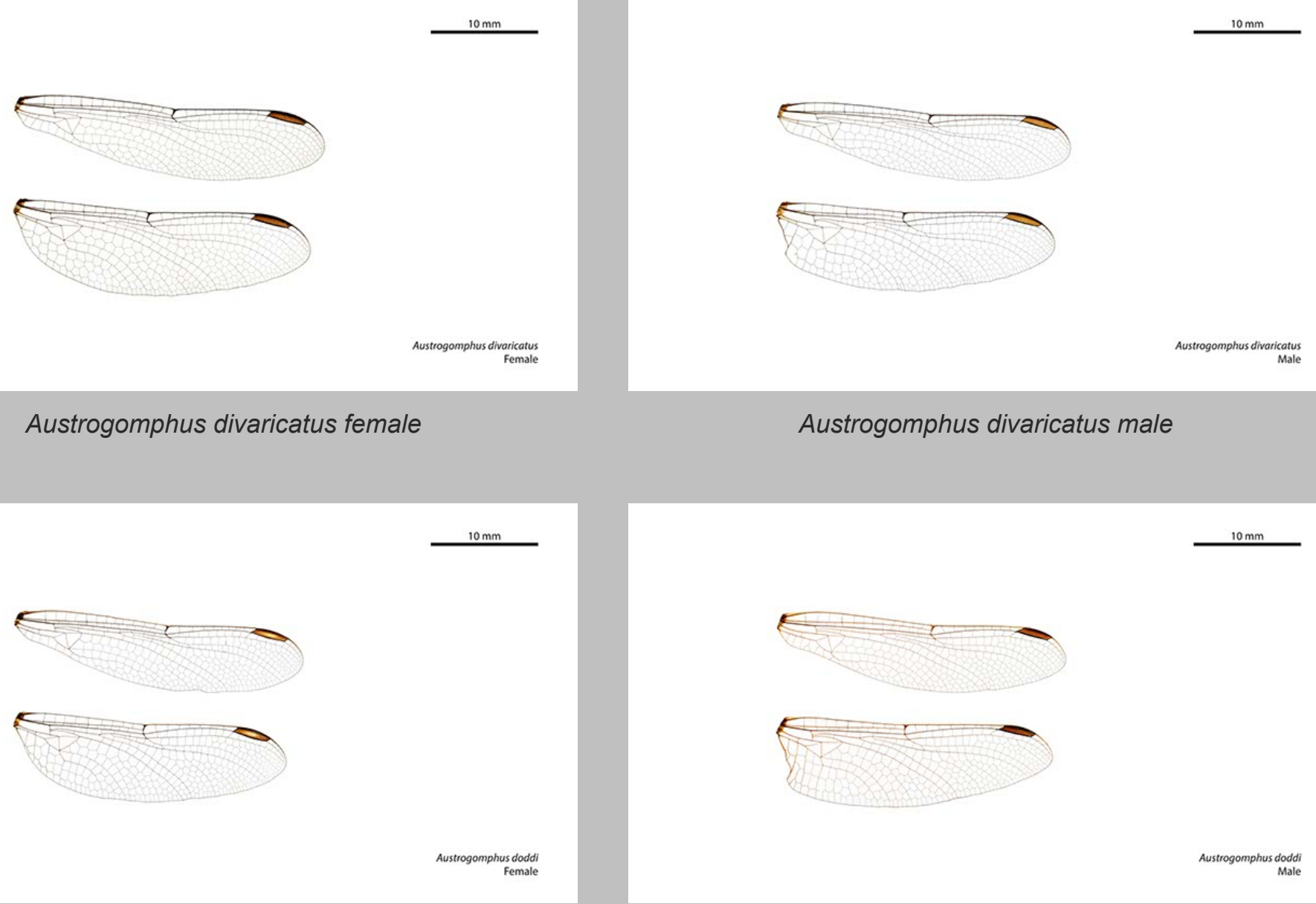

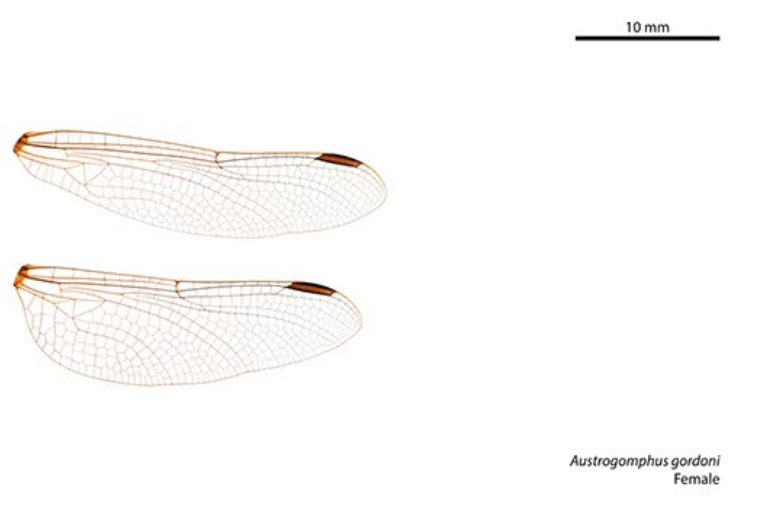

Austrogomphus gordoni female

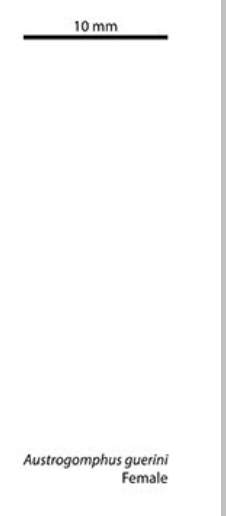

Austrogomphus guerini female
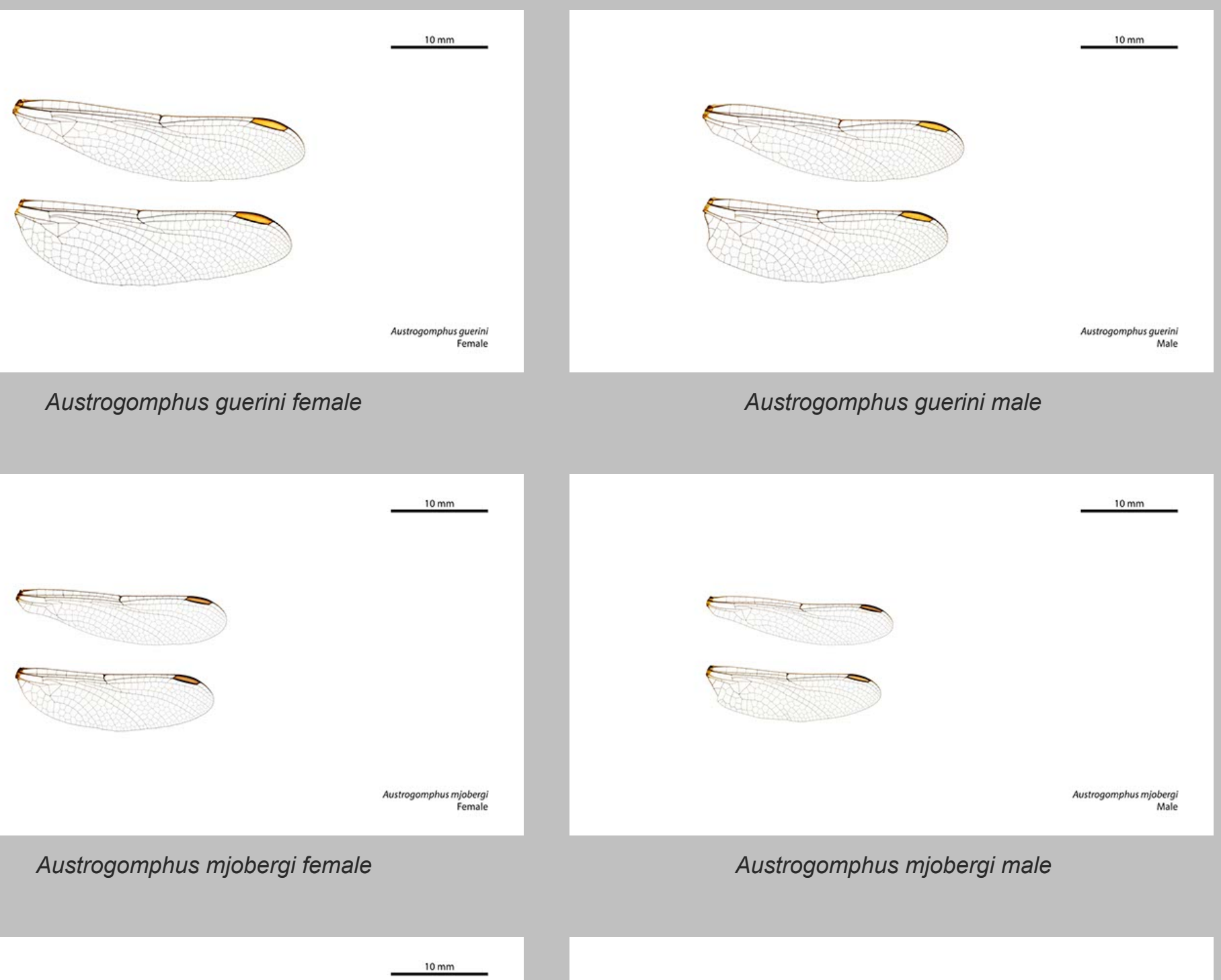

Austrogomphus gordoni male

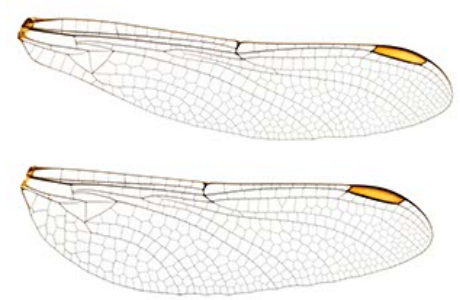

Austrogomphus guerini male

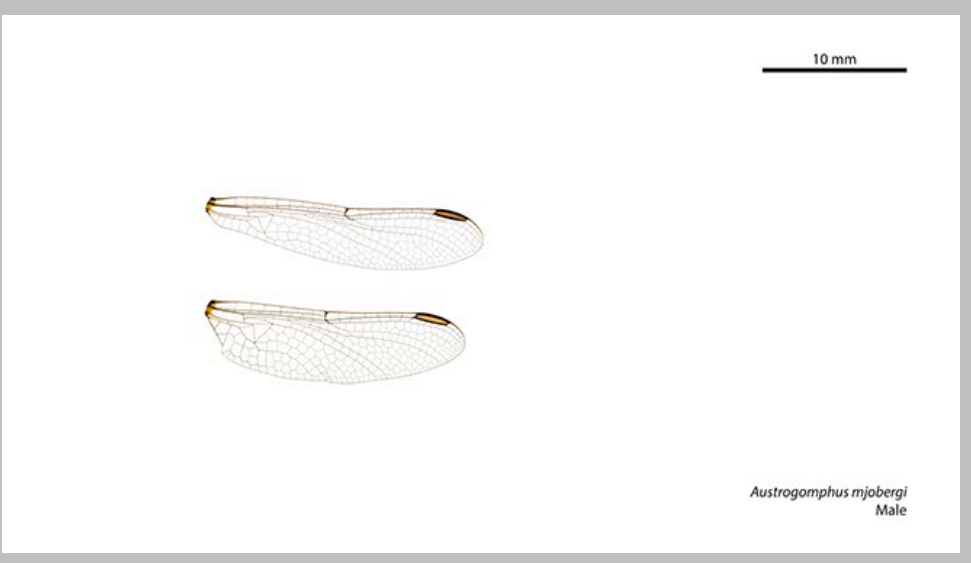

Austrogomphus mjobergi male 

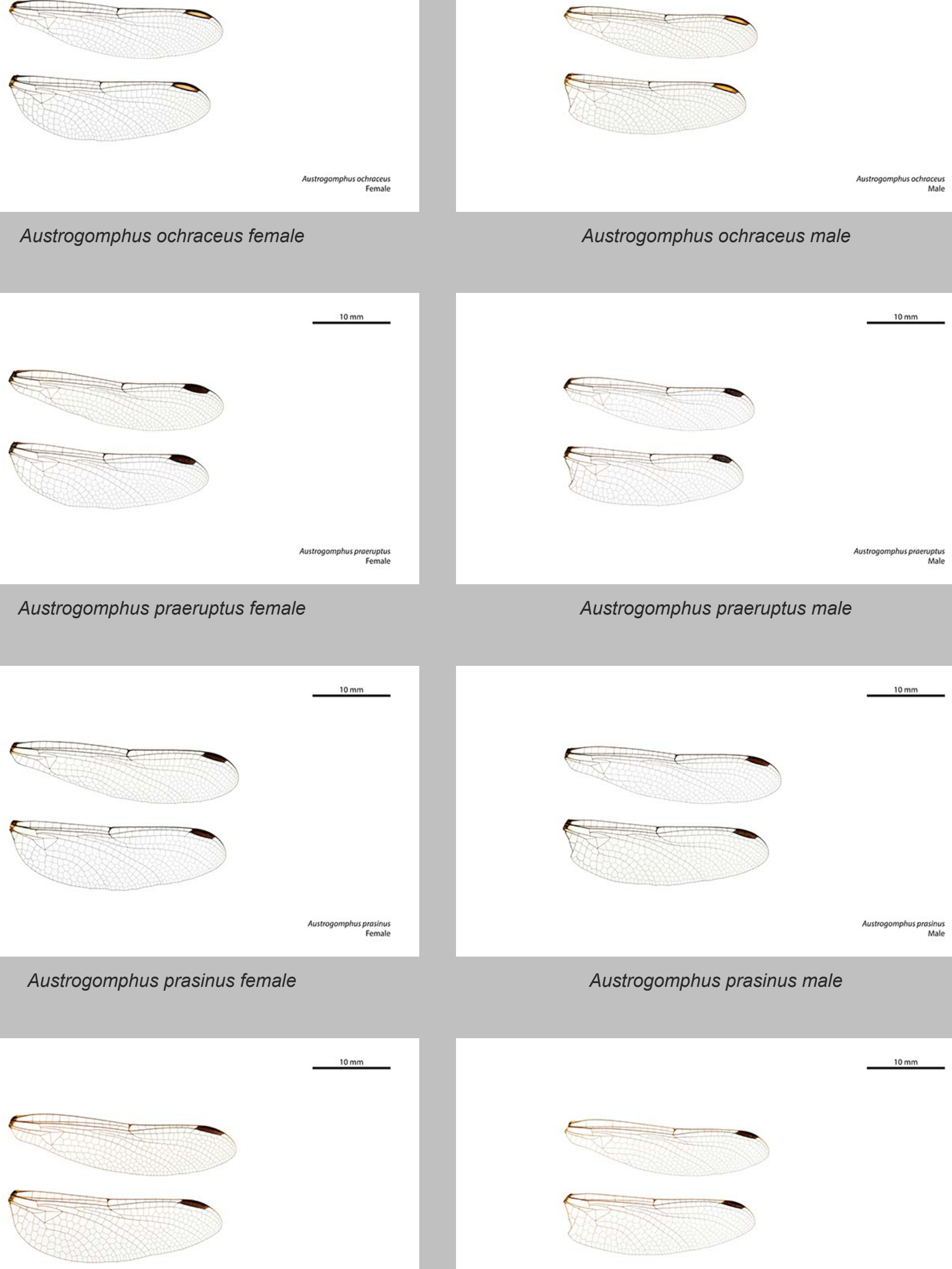

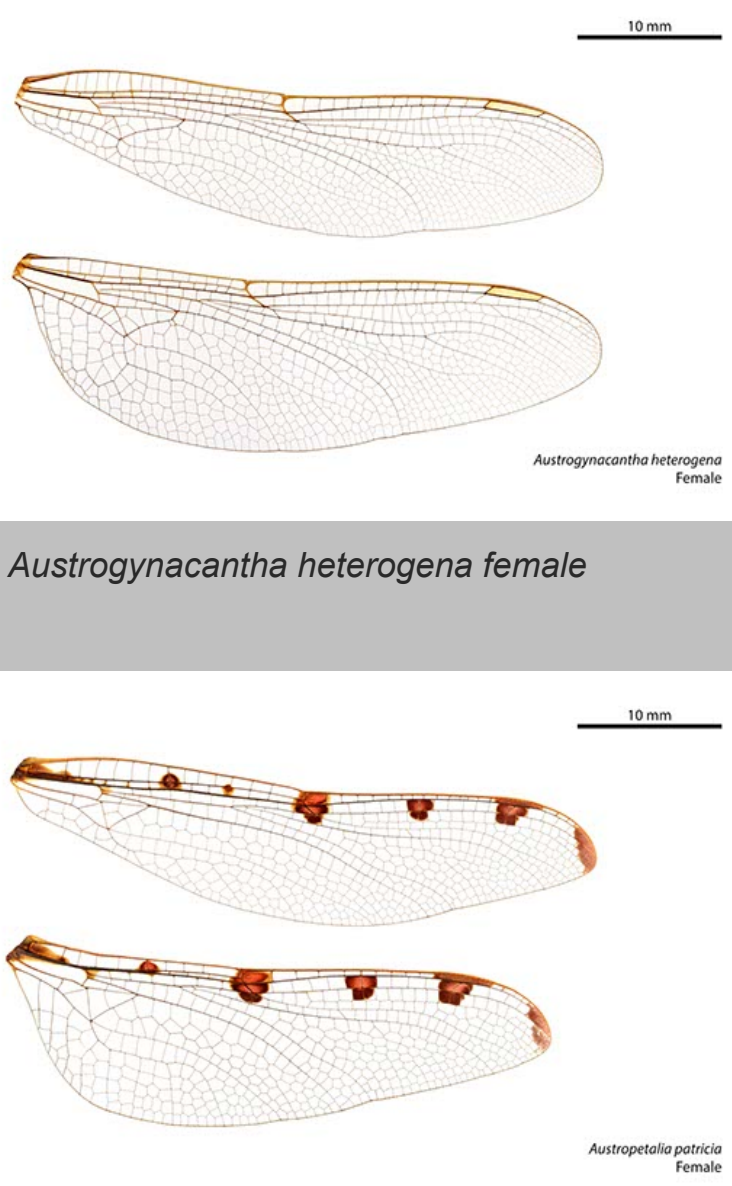

Austropetalia patricia female

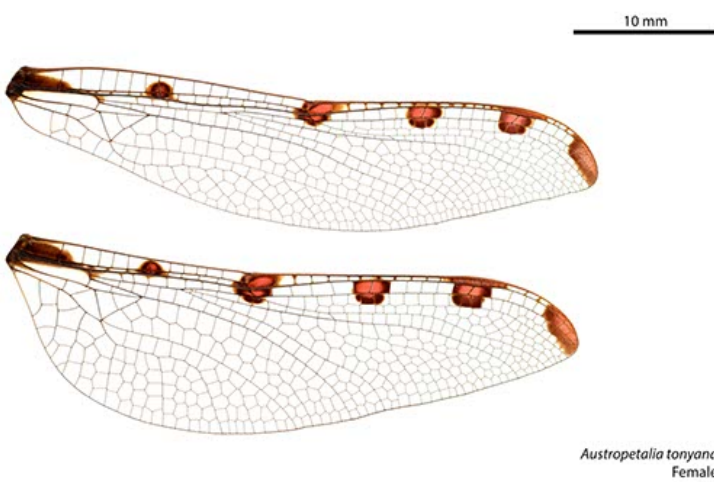

Austropetalia tonyana female

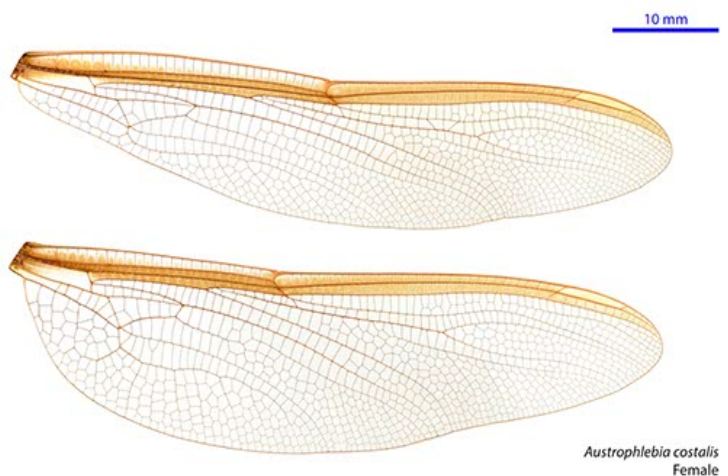

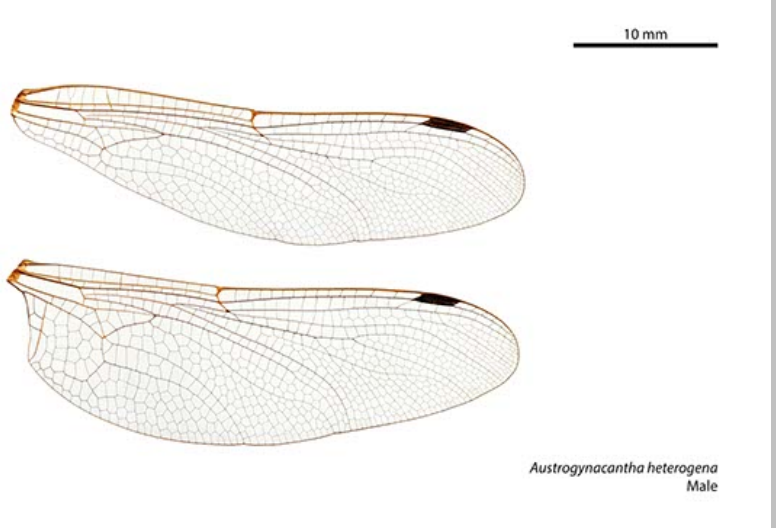

Austrogynacantha heterogena male

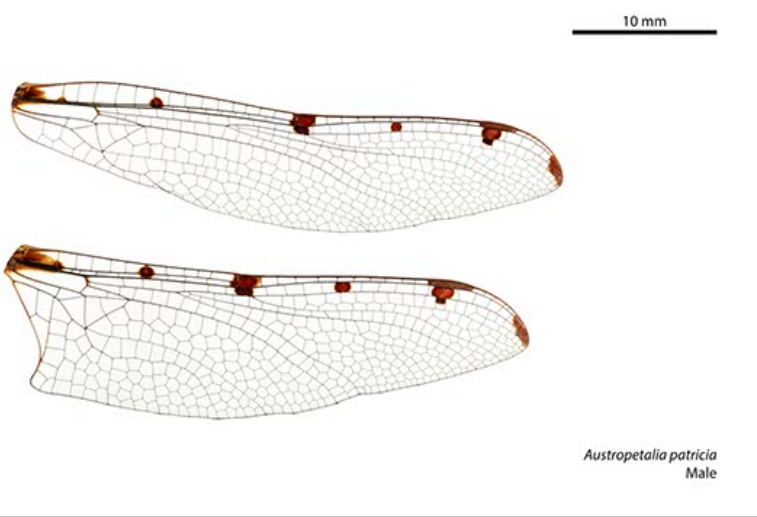

Austropetalia patricia male

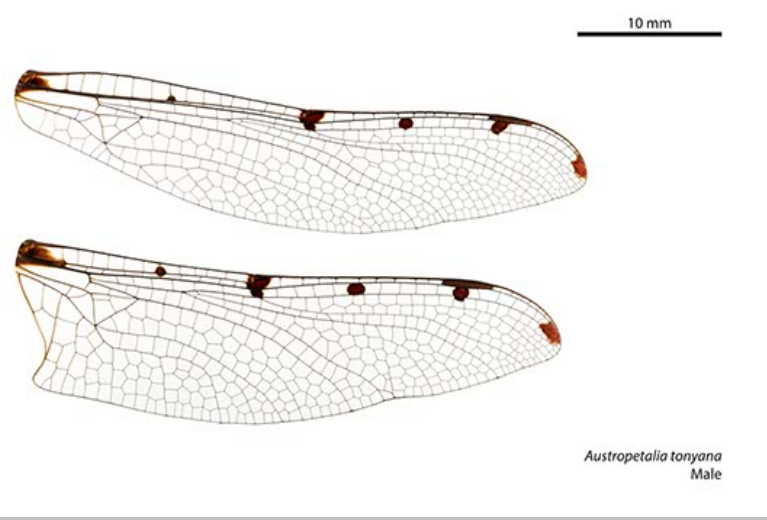

Austropetalia tonyana male

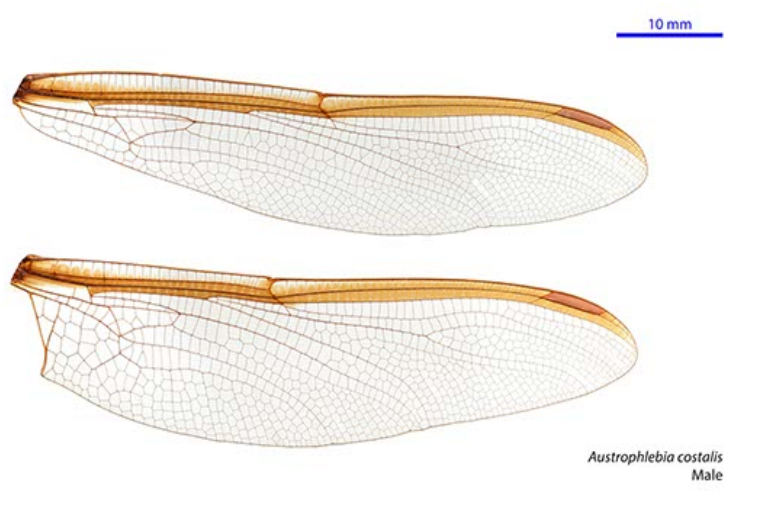

Austrophlebia costalis male 


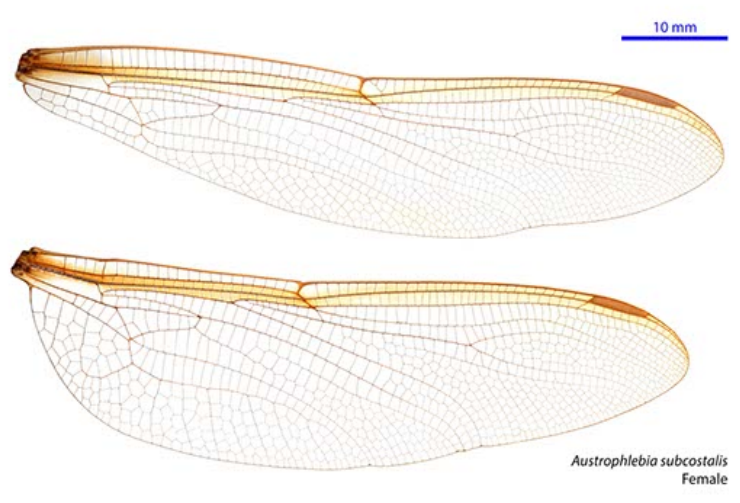

Austrophlebia subcostalis female

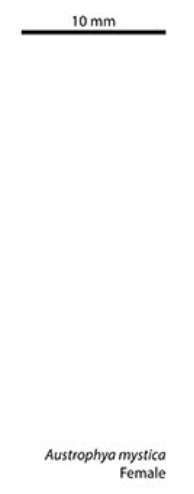

Austrophya mystica female

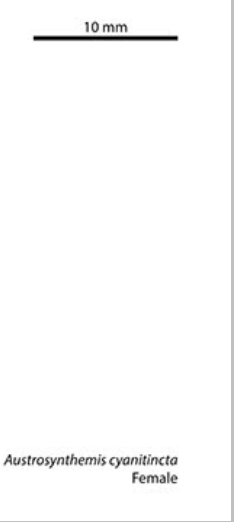

Austrosynthemis cyanitincta female

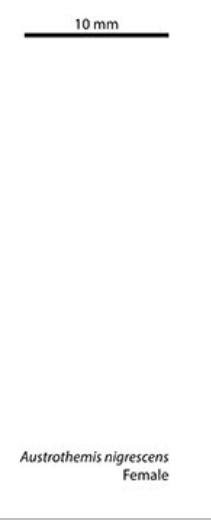




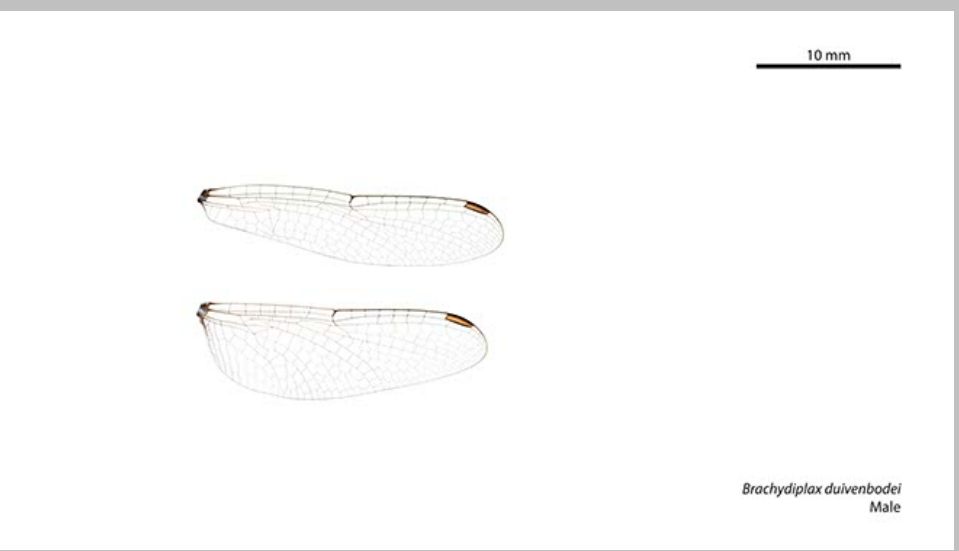

Brachydiplax duivenbodei female

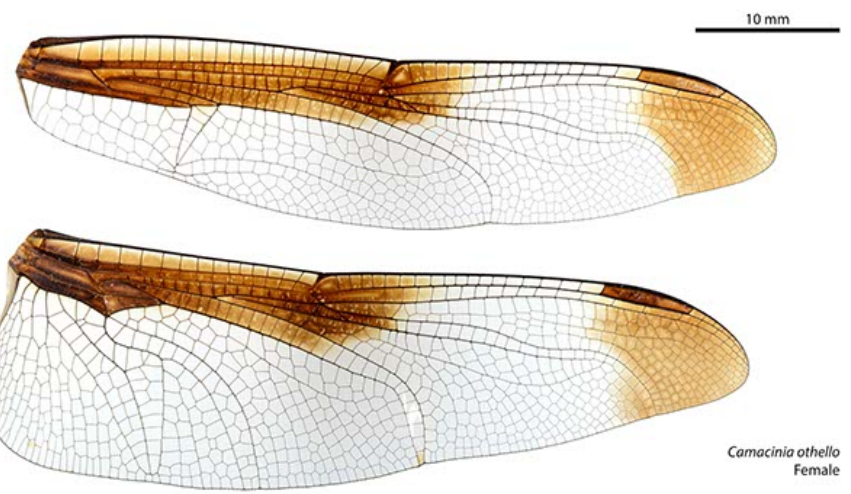

Camacinia othello female

\section{Brachydiplax duivenbodei male}

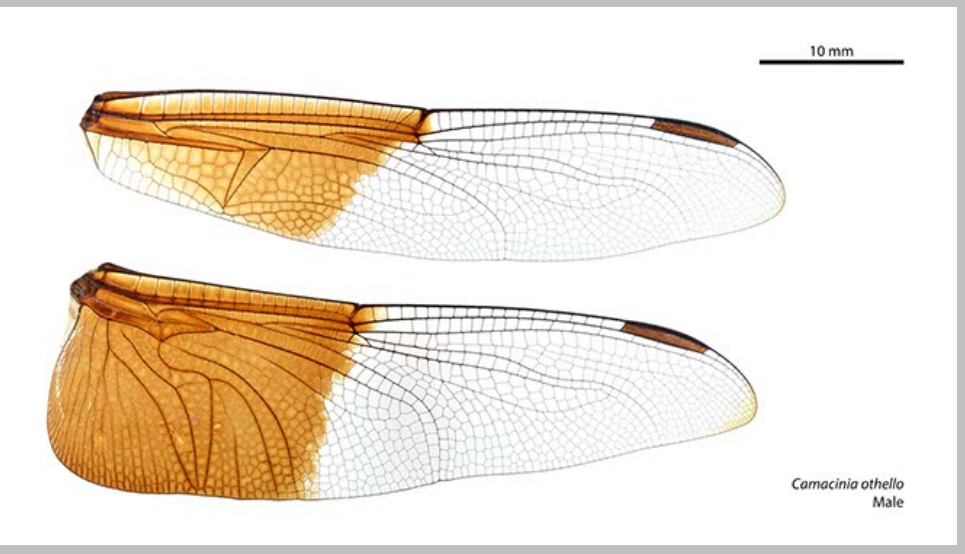

Camacinia othello male
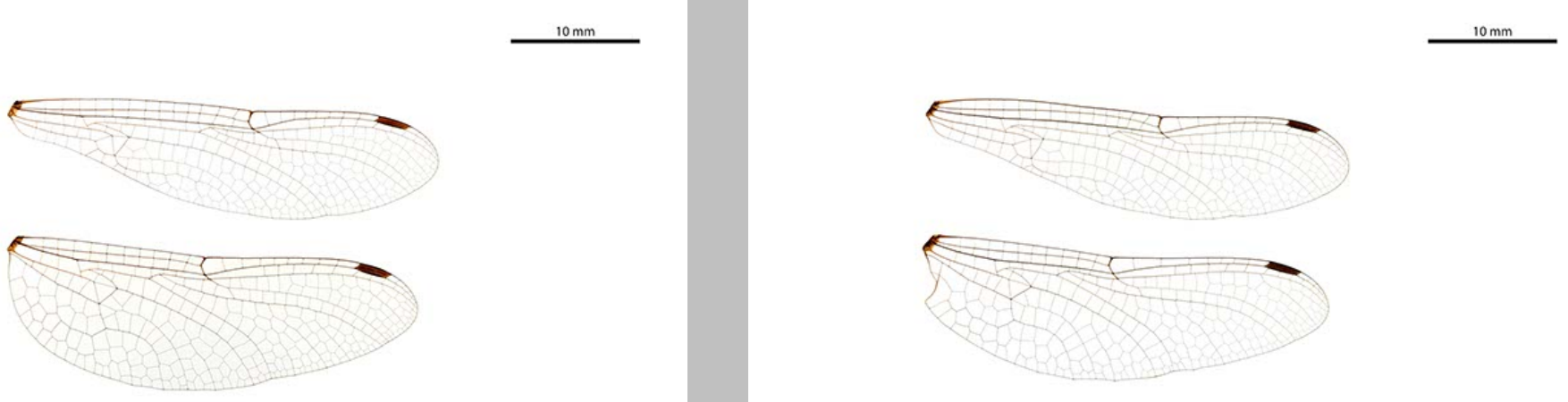


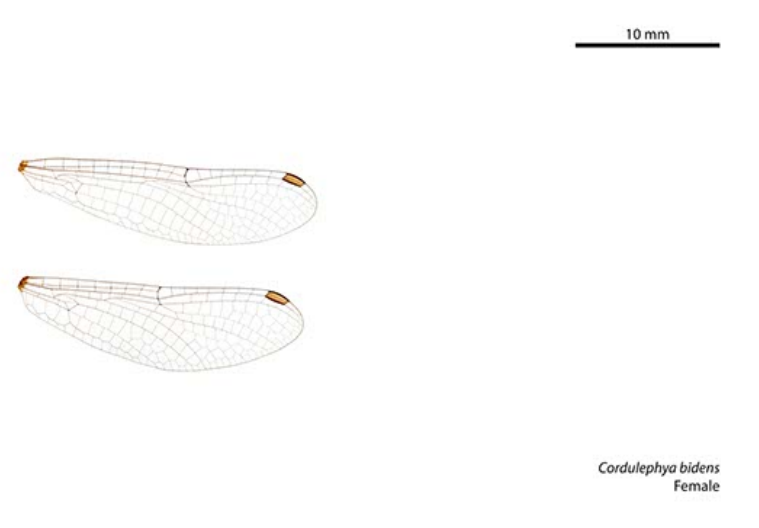

Cordulephya bidens female

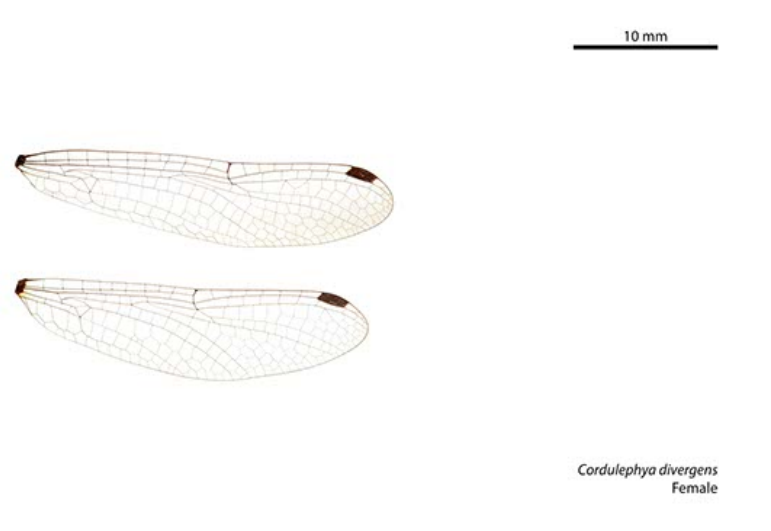

Cordulephya divergens female

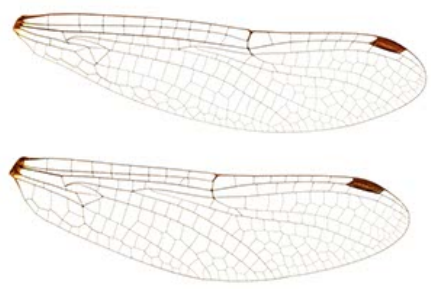

Cordulephya montana
Female

Cordulephya montana female

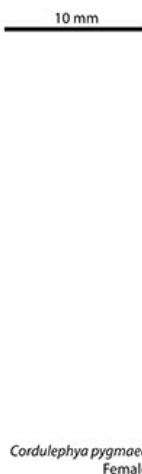

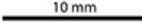

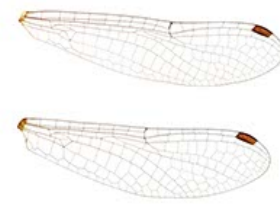

Cordulephya bidens male

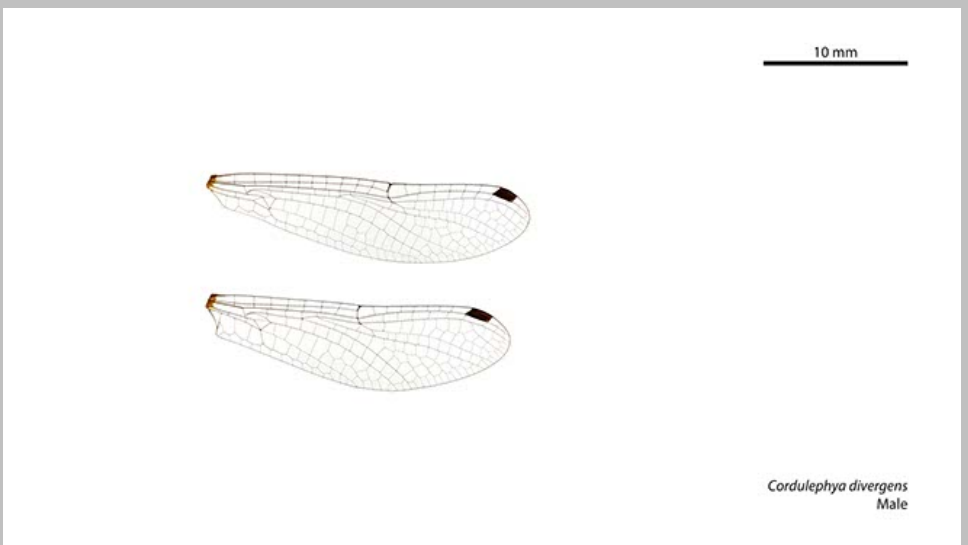

Cordulephya divergens male

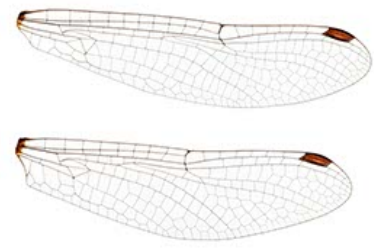

Cordulephya montana male

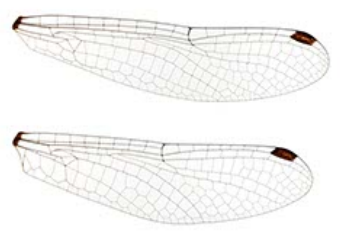




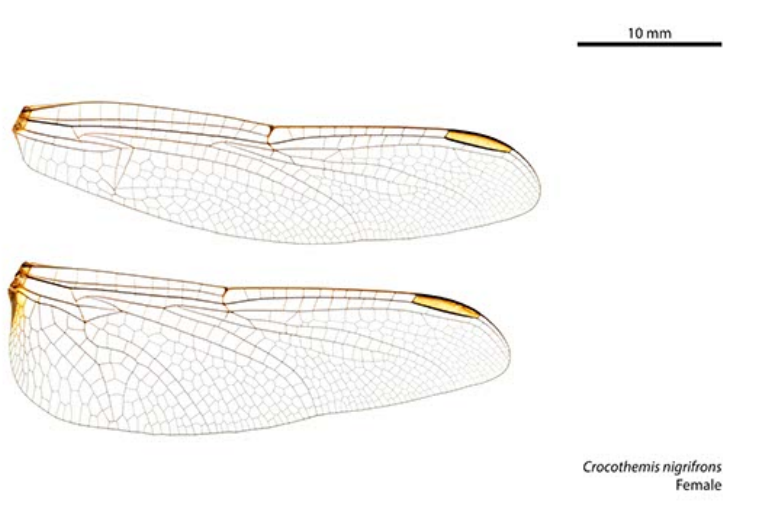

Crocothemis nigrifrons female

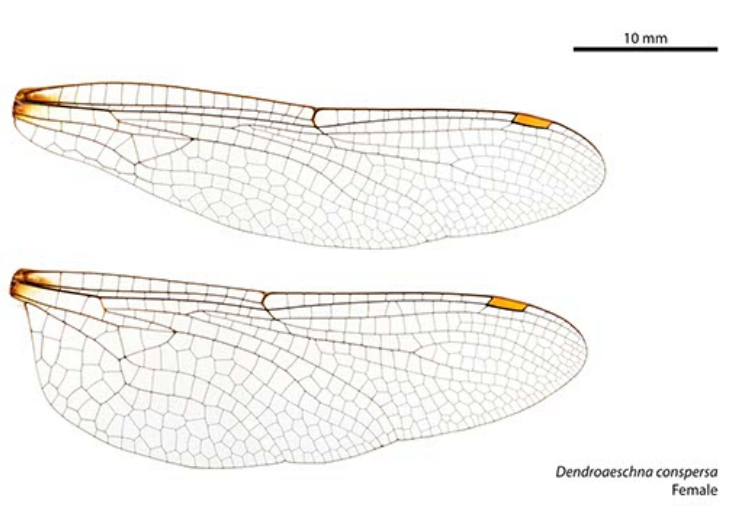

Dendroaeschna conspersa female

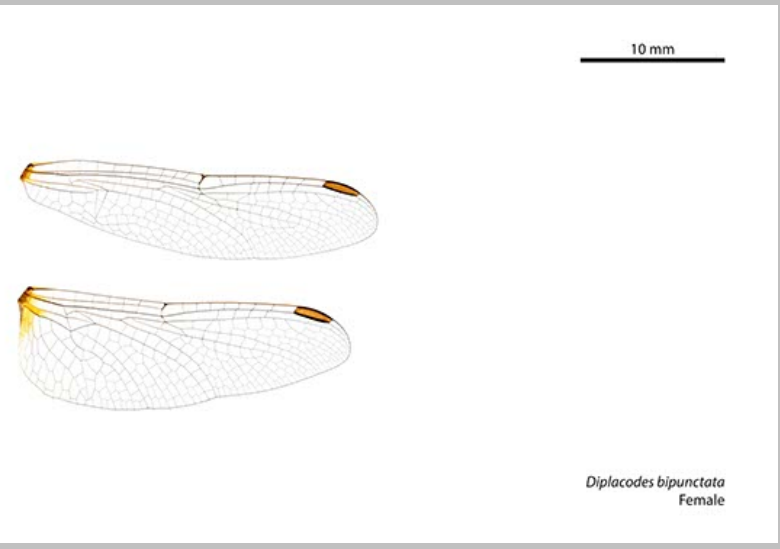

Diplacodes bipunctata female
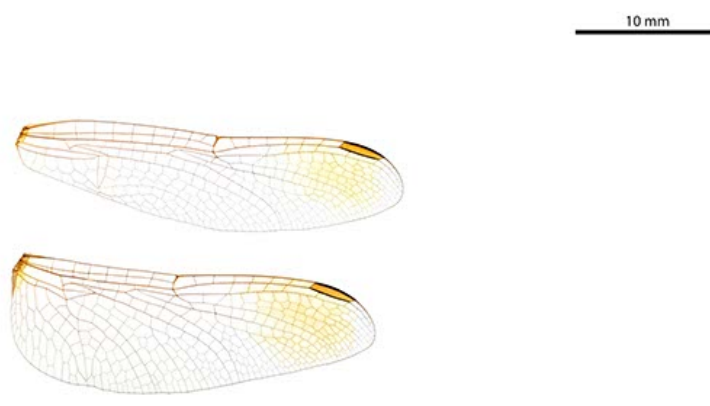

Diplacodes haematodes

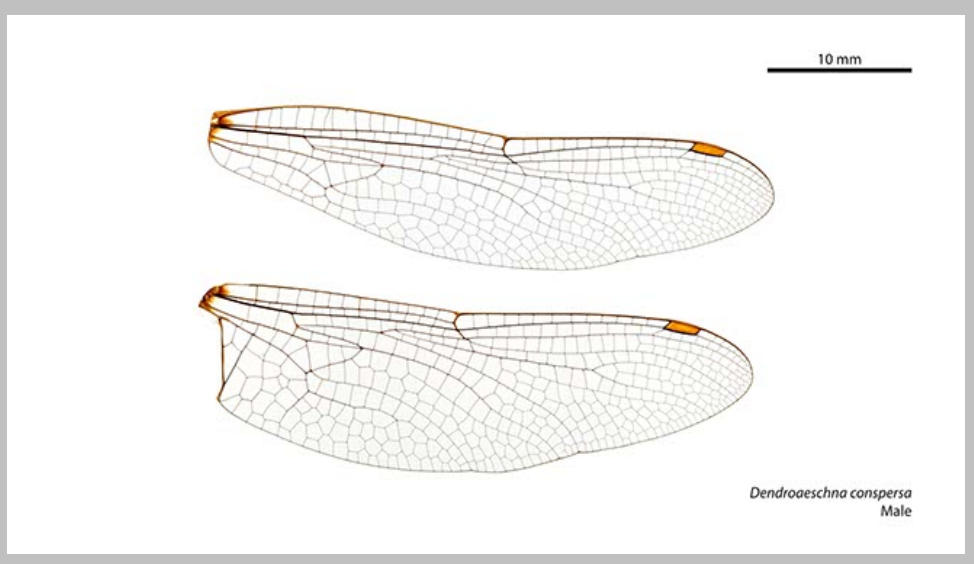

Dendroaeschna conspersa male

Crocothemis nigrifrons male

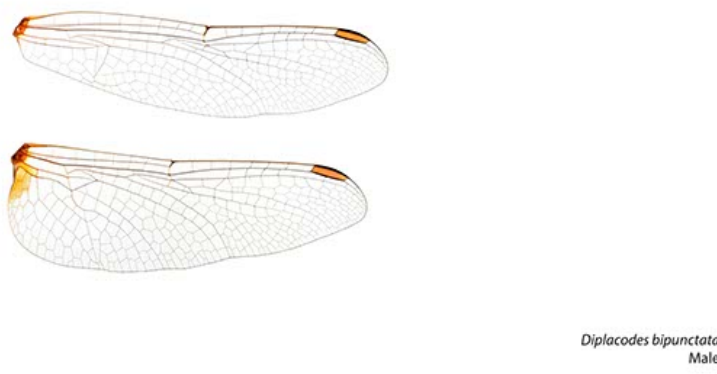

Diplacodes bipunctata male

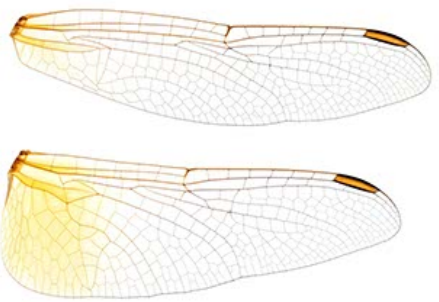

Diplacodes haematodes 

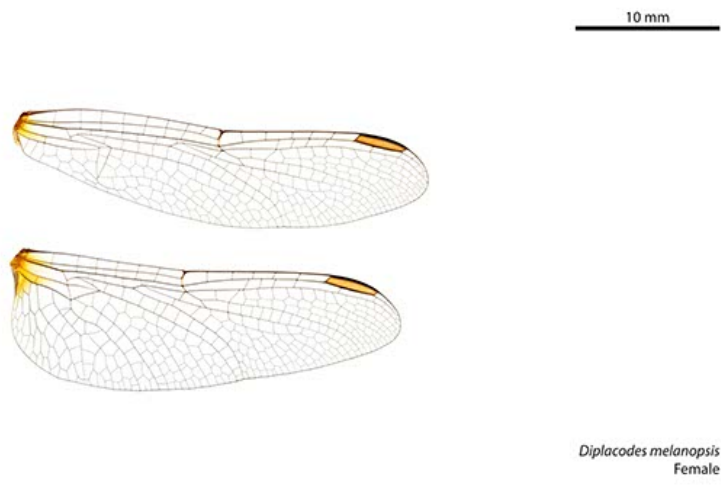

Diplacodes melanopsis female

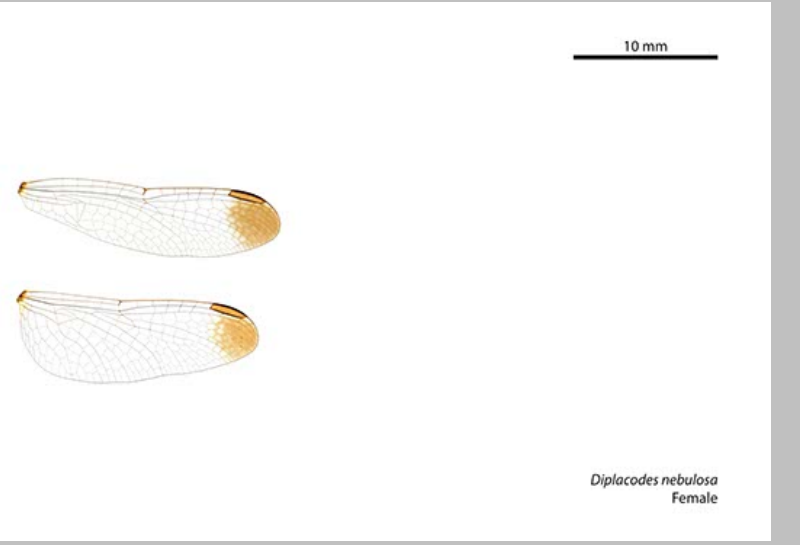

Diplacodes nebulosa female
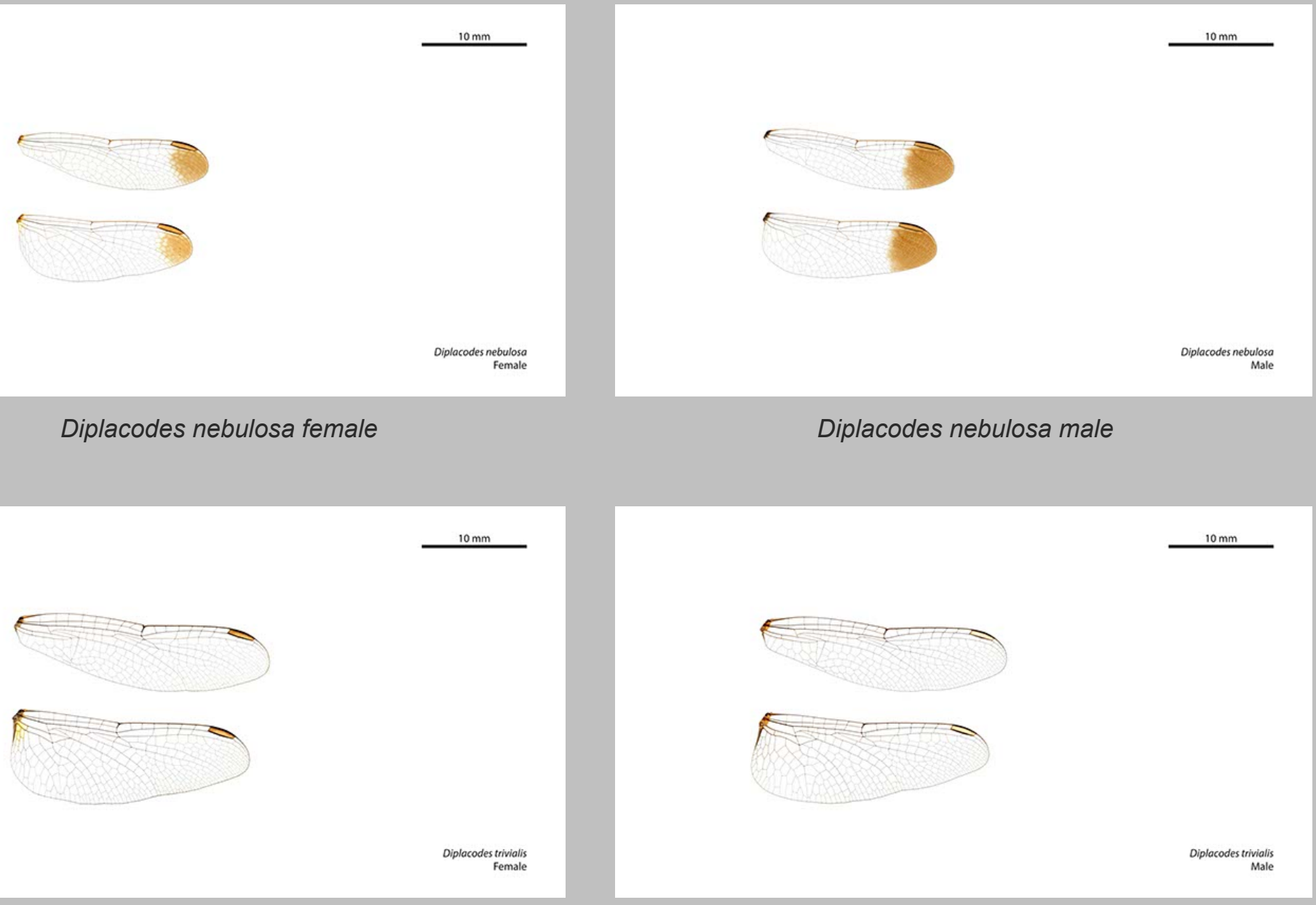

Diplacodes melanopsis male

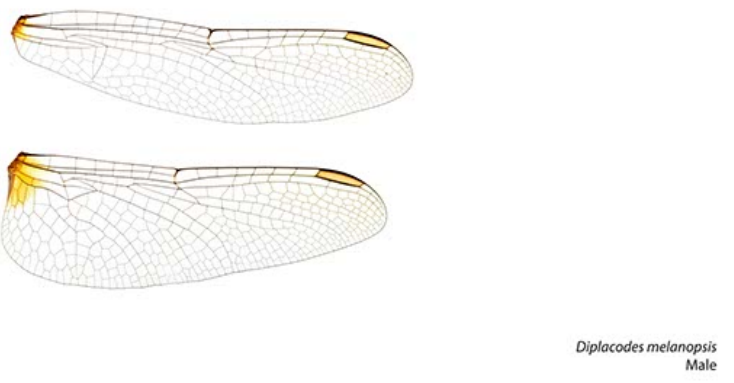

Diplacodes nebulosa male

Diplacodes trivialis female

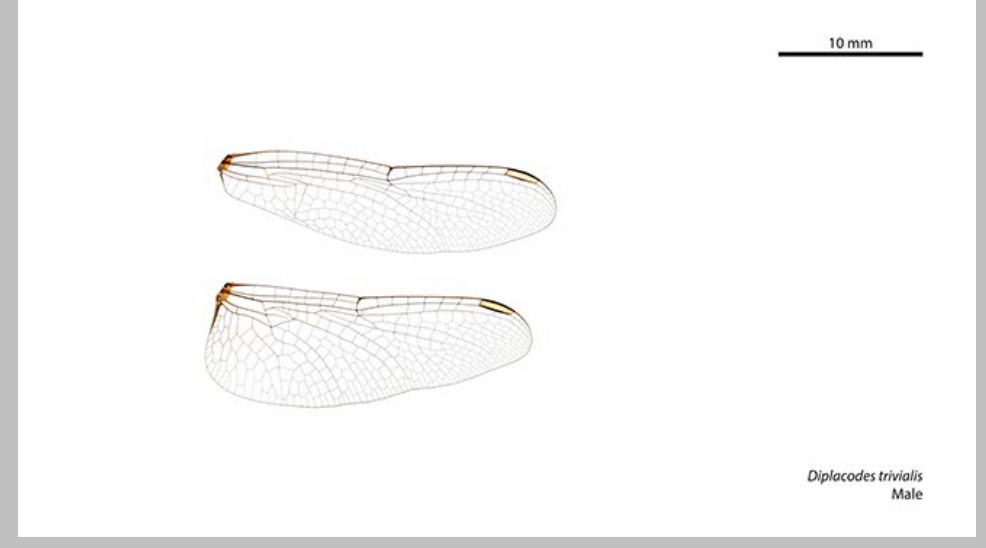

Diplacodes trivialis male
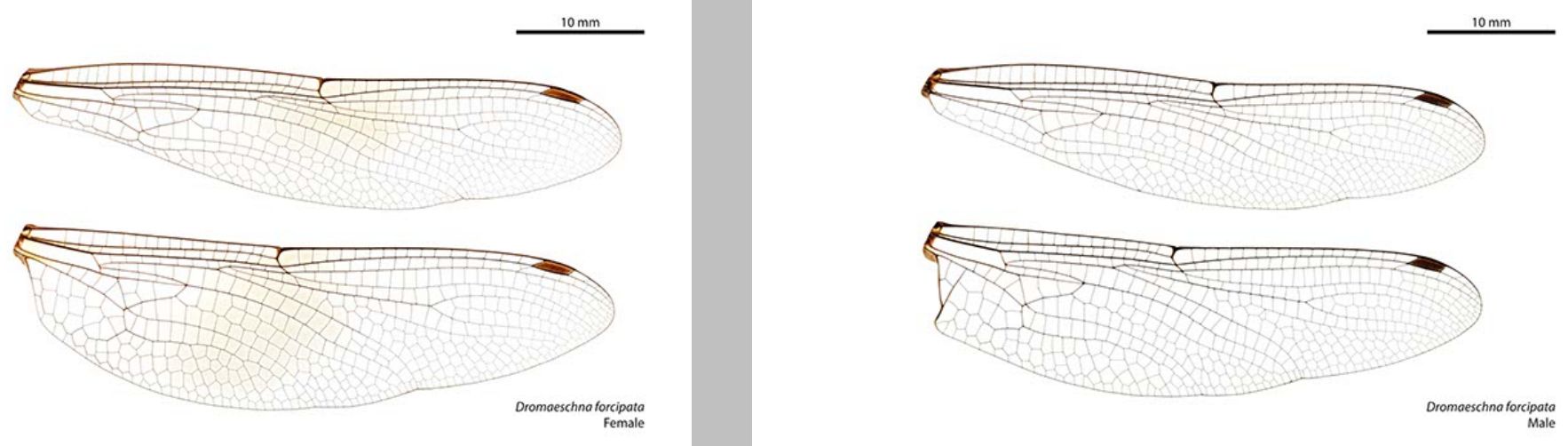


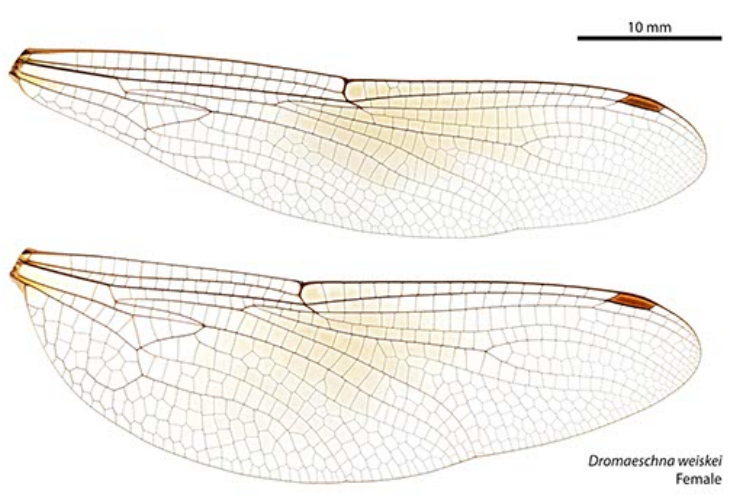

Dromaeschna weiskei female

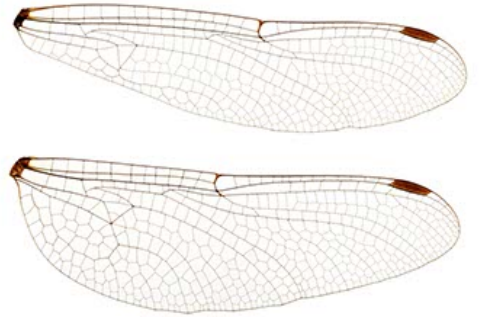

Eusynthemis ourolineato
Female

Eusynthemis aurolineata female

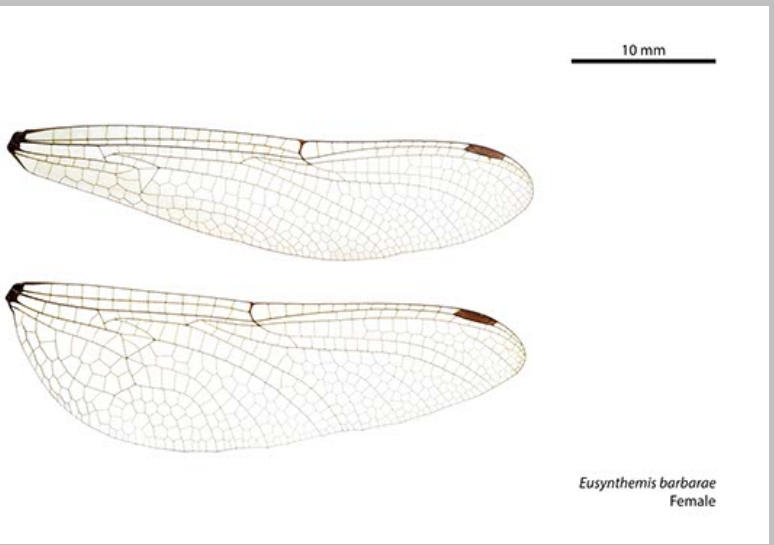

Eusynthemis barbarae female

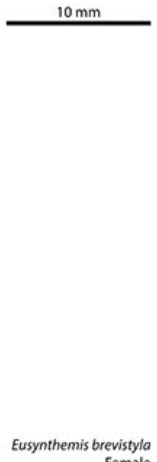

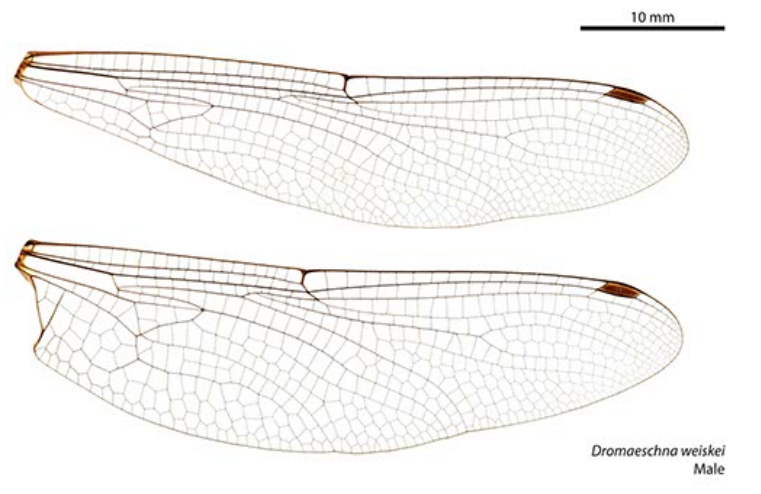

Dromaeschna weiskei male

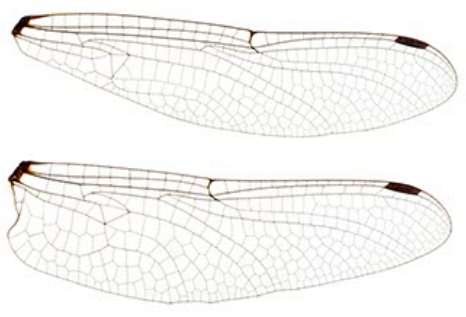

Eusynthemis aurolineato
Male

Eusynthemis aurolineata male

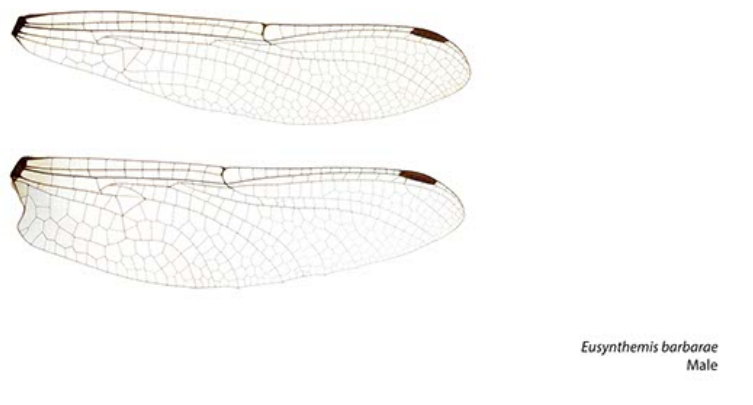

Eusynthemis barbarae male

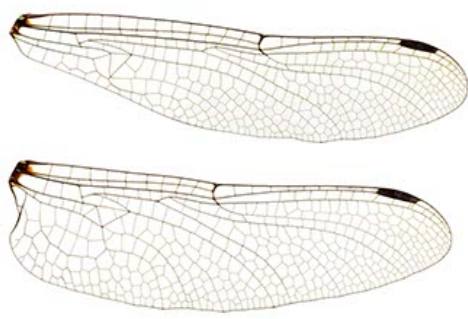

Eusynthemis brevistyla 

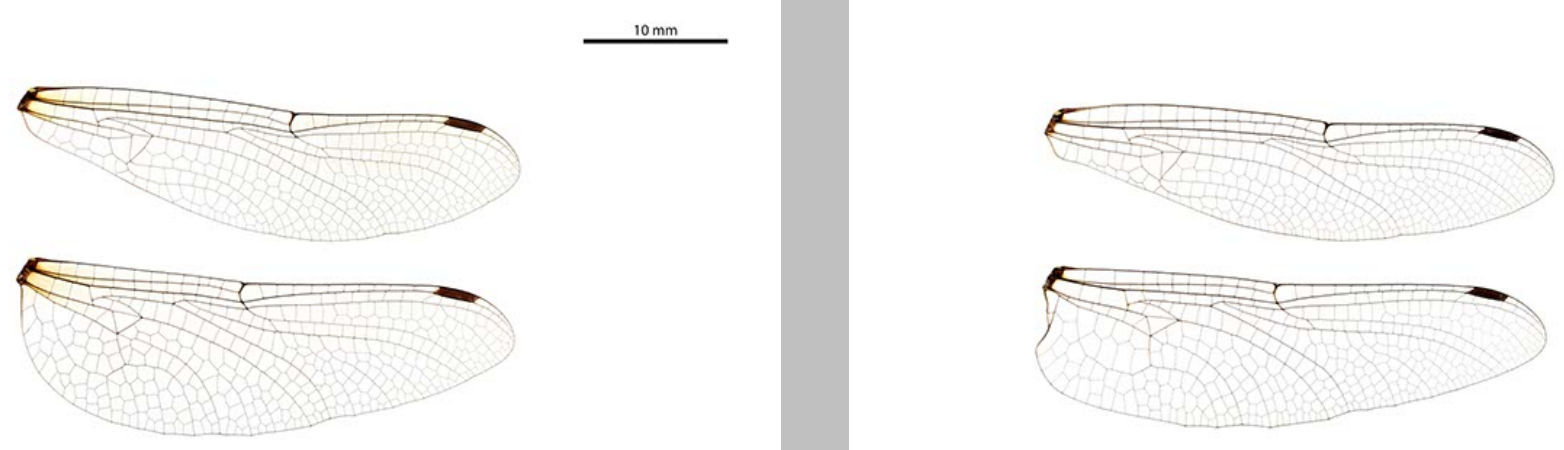

\section{Eusynthemis deniseae male}
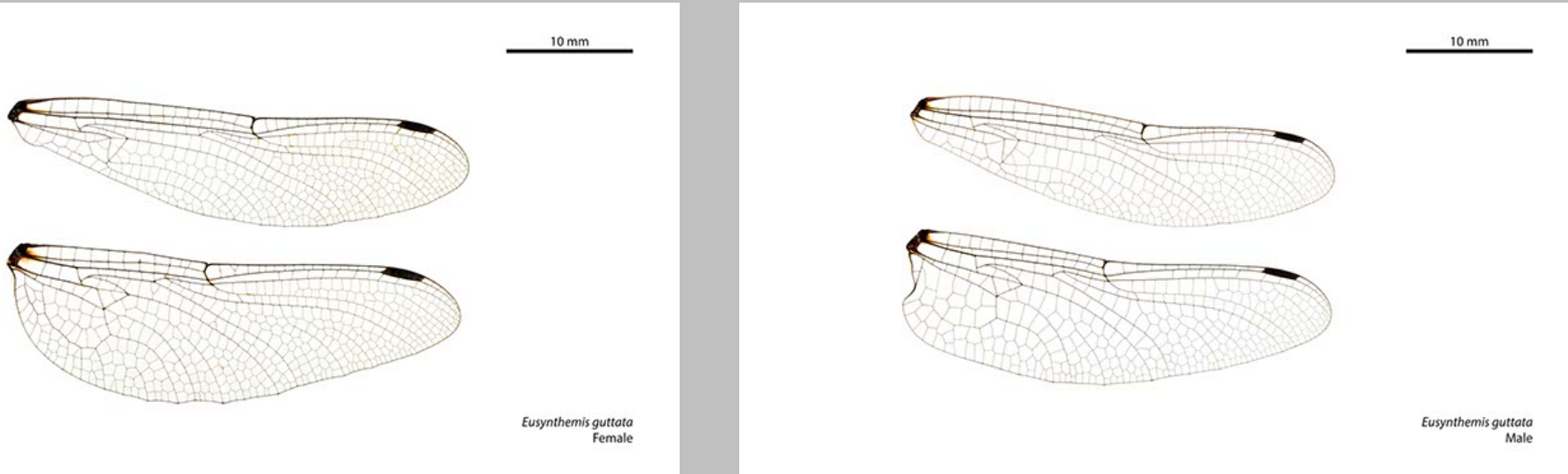

Eusynthemis guttata male

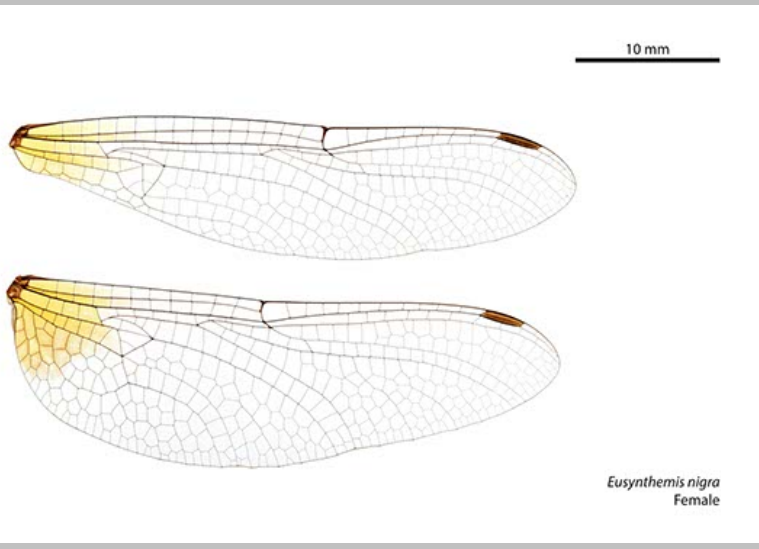

Eusynthemis nigra female

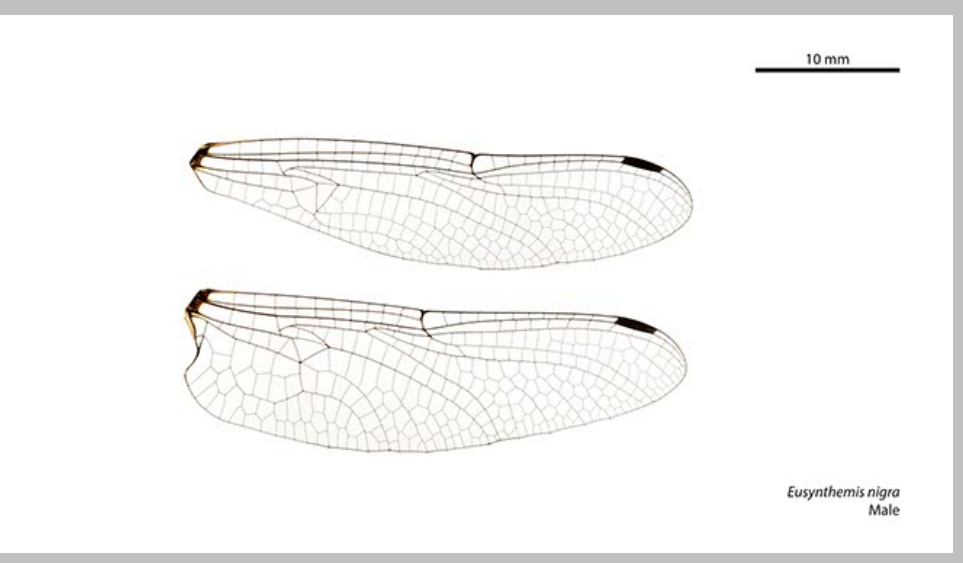

Eusynthemis nigra male

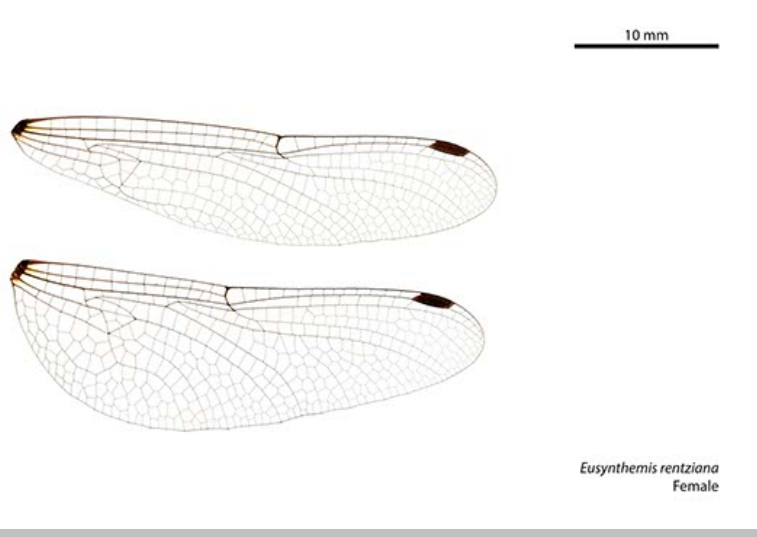


Eusynthemis tenera female

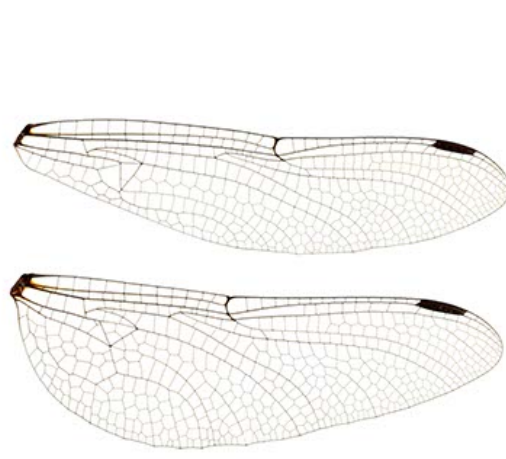

Eusynthemis tillyardi
Female
Eusynthemis tenera male

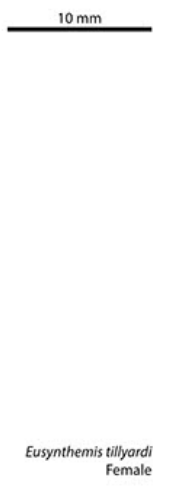

Eusynthemis tillyardi female

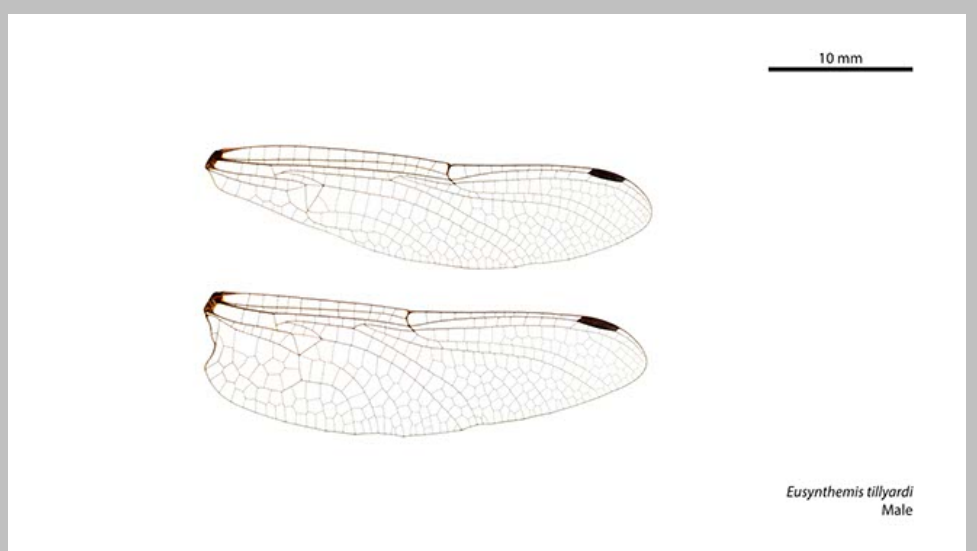

Eusynthemis tillyardi male

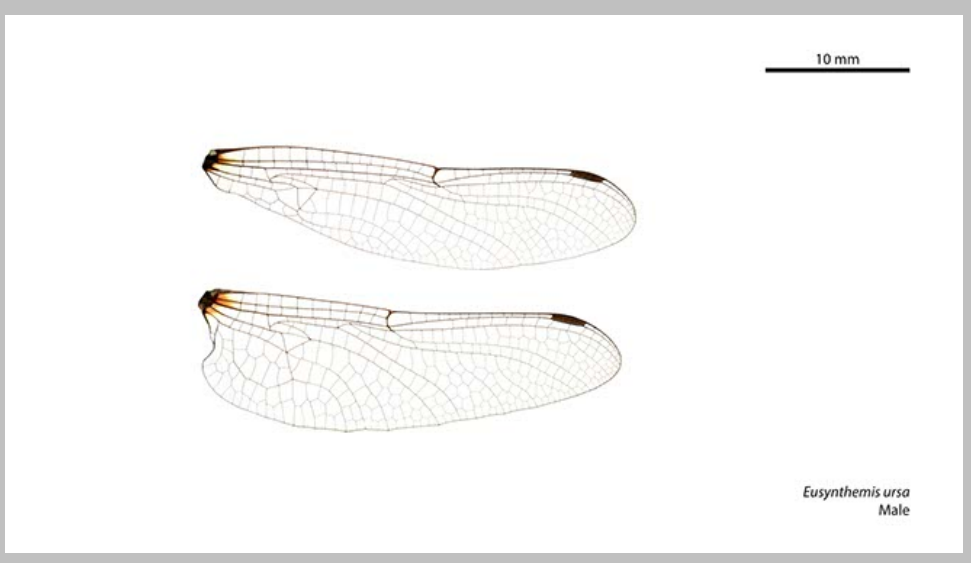

Eusynthemis ursa male

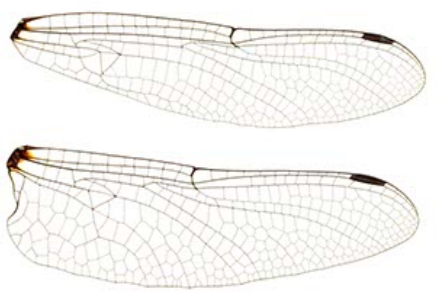



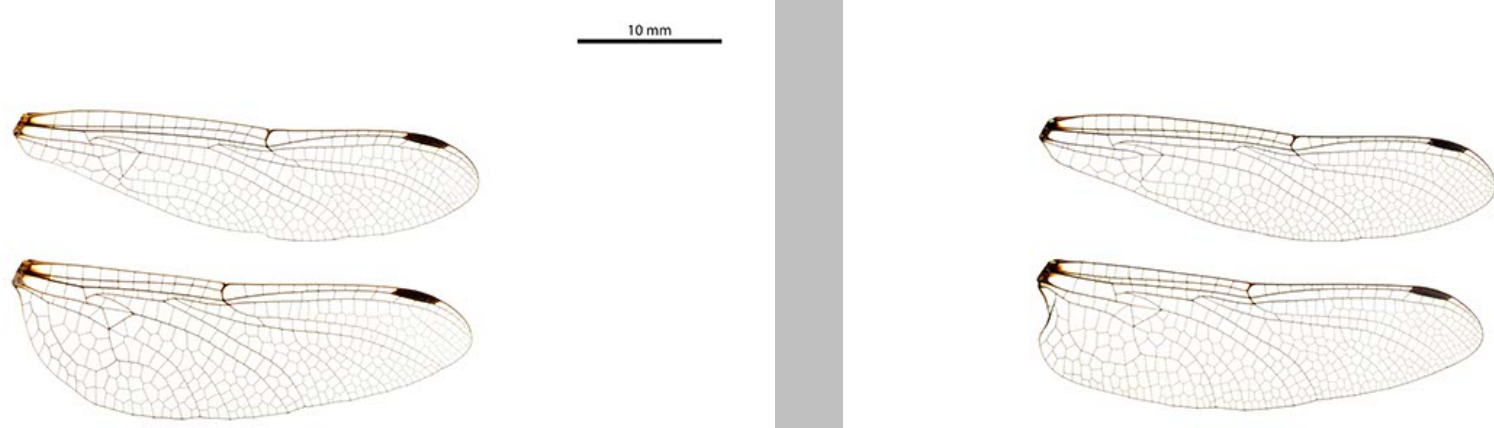

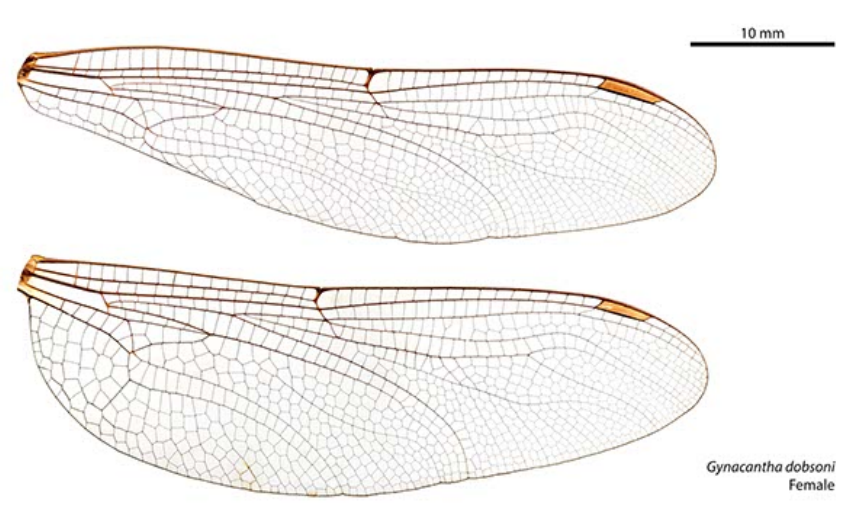

Gynacantha dobsoni female

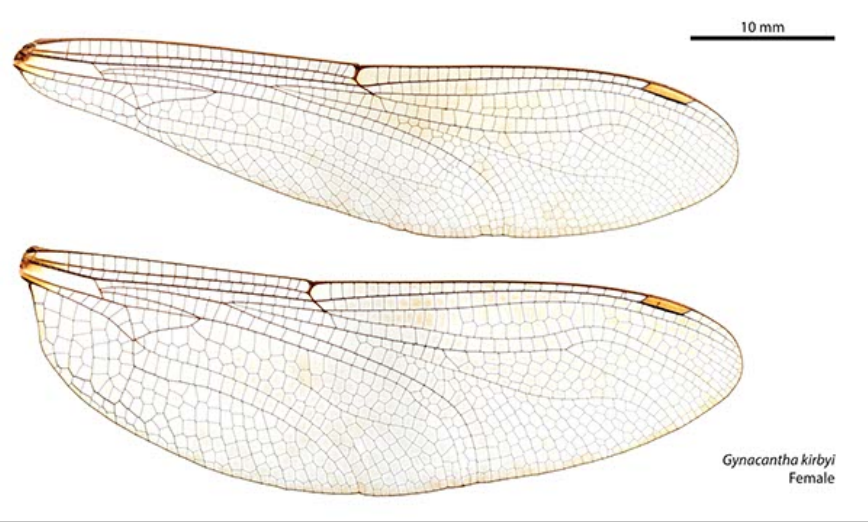

Gynacantha kirbyi female

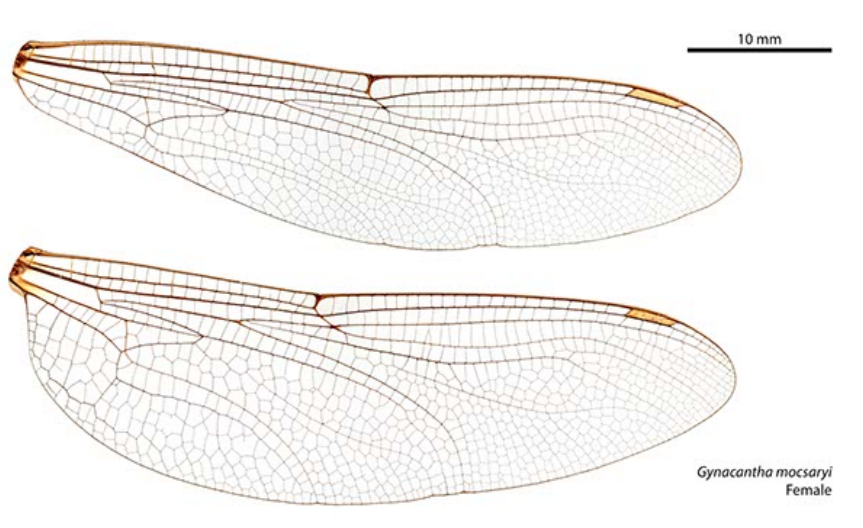

Gynacantha mocsaryi female

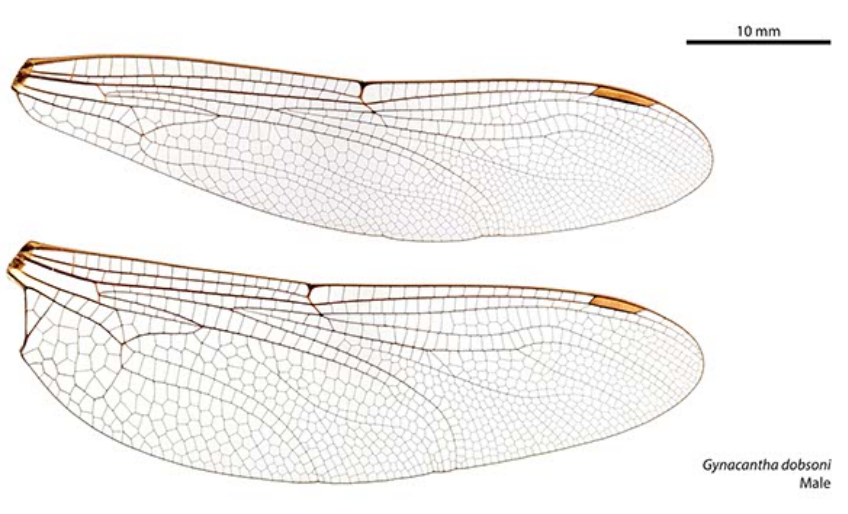

Gynacantha dobsoni male

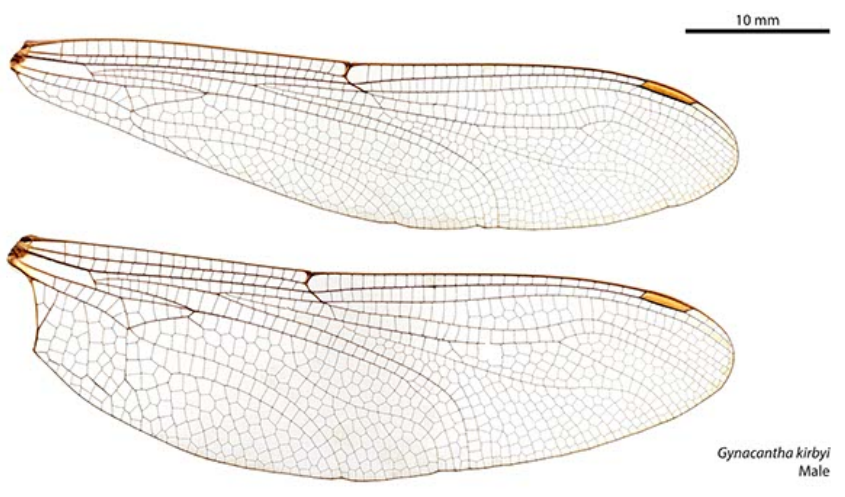

Gynacantha kirbyi male

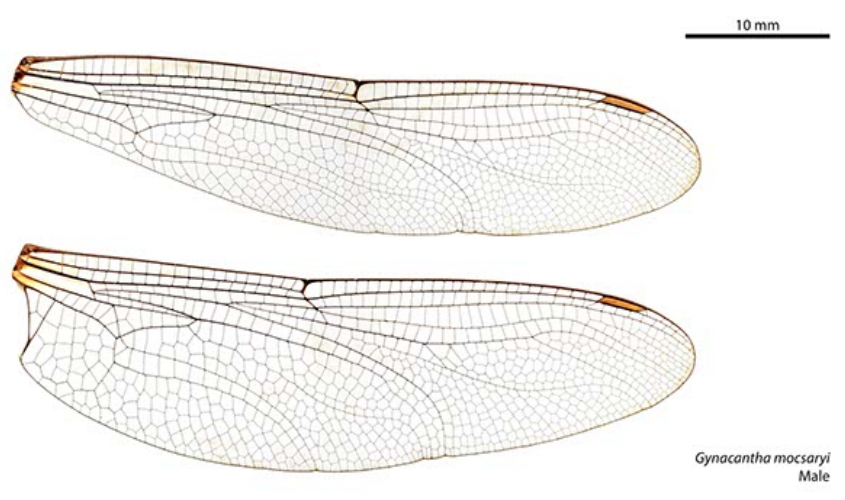

Gynacantha mocsaryi male 


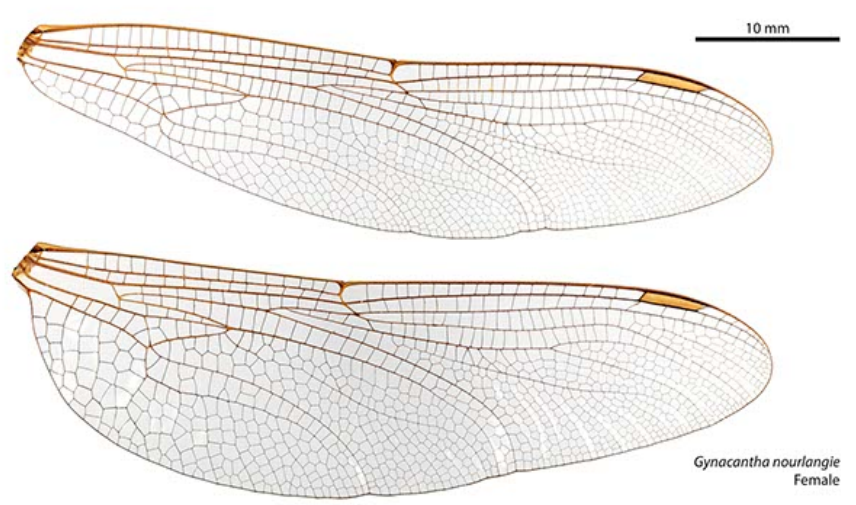

Gynacantha nourlangie female

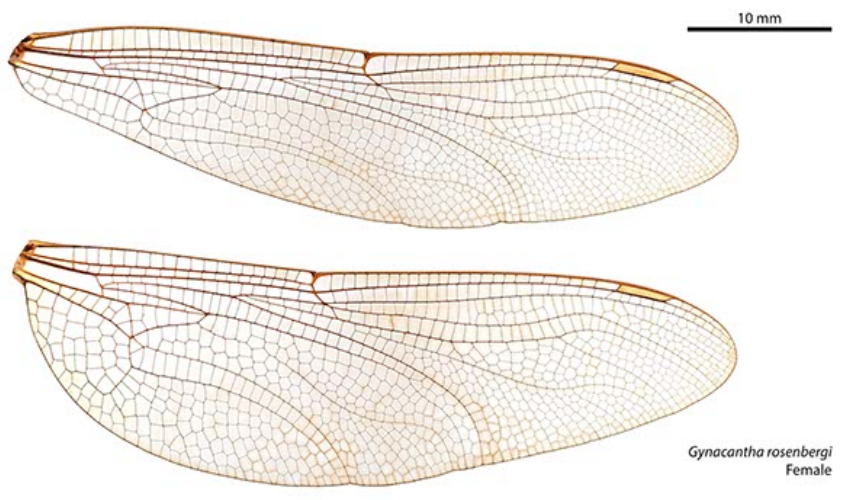

Gynacantha rosenbergi female

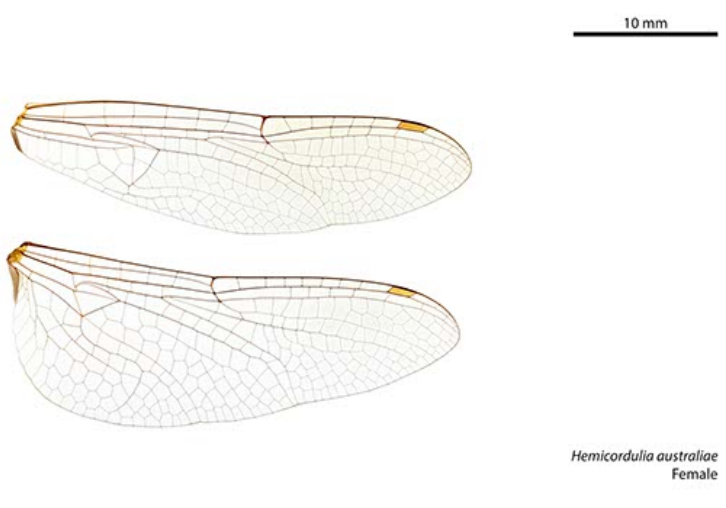

Hemicordulia australiae female
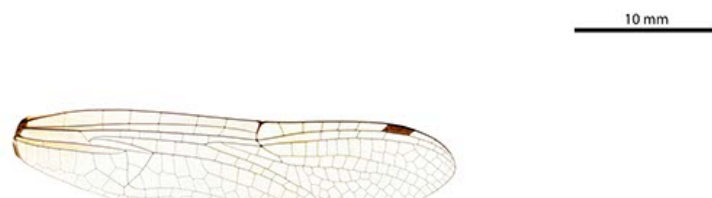

5

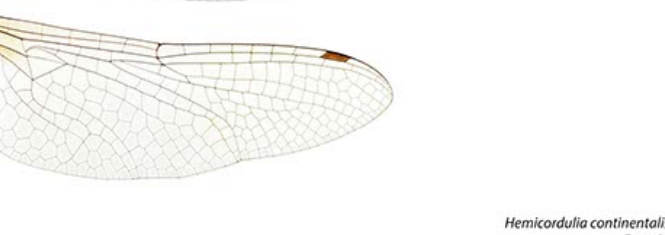

Gynacantha rosenbergi male
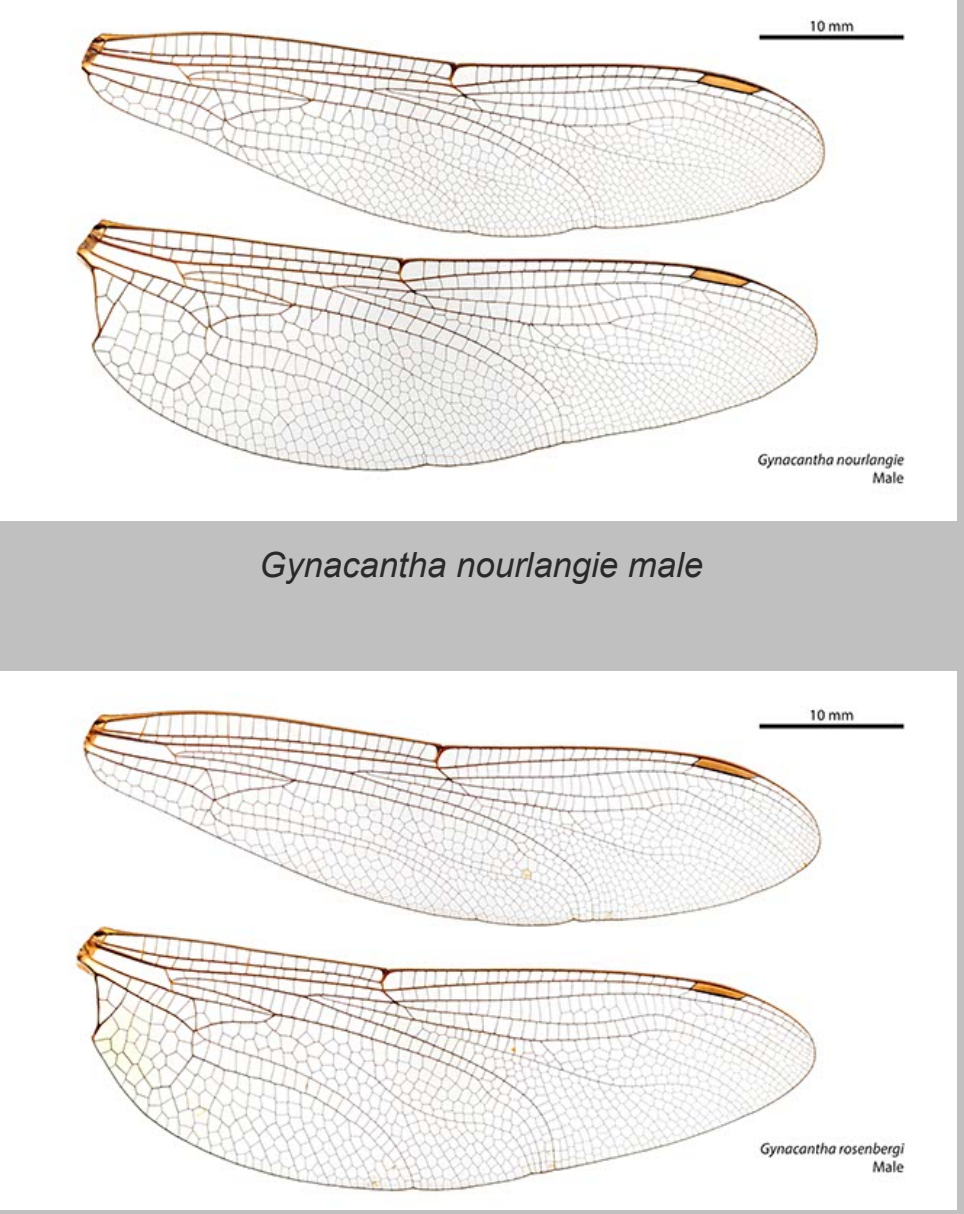

Gynacantha nourlangie male 


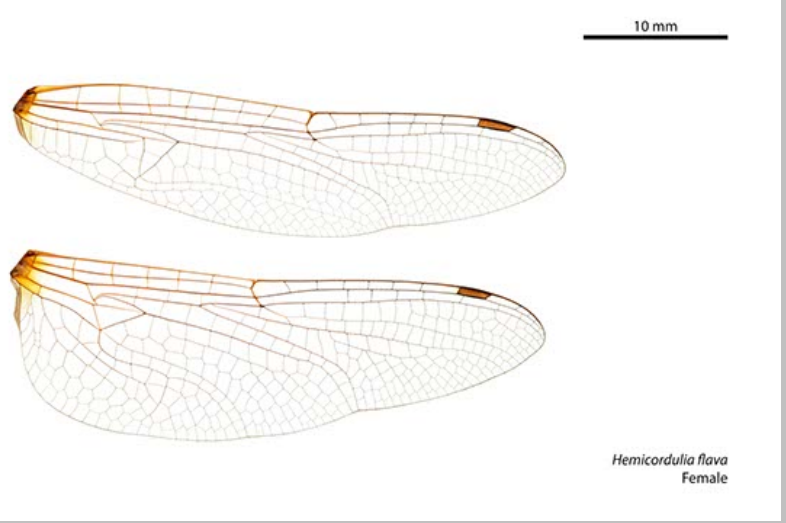

Hemicordulia flava female

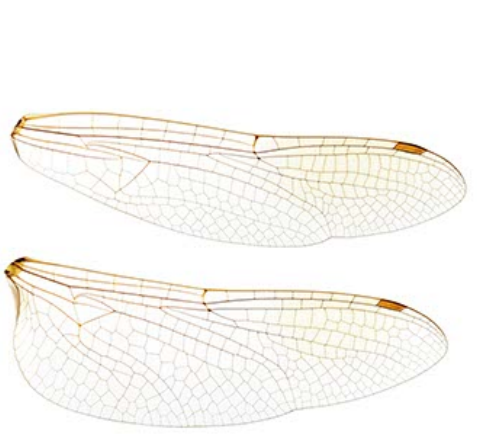

Hemicordulia intermedia female

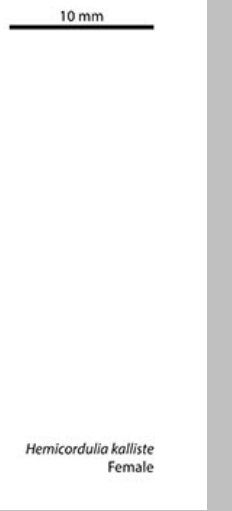

Hemicordulia kalliste female

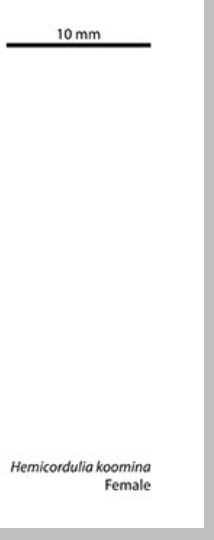

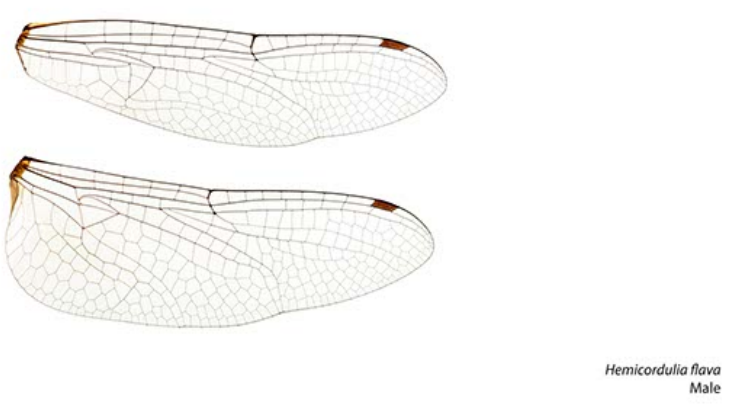

Hemicordulia flava male

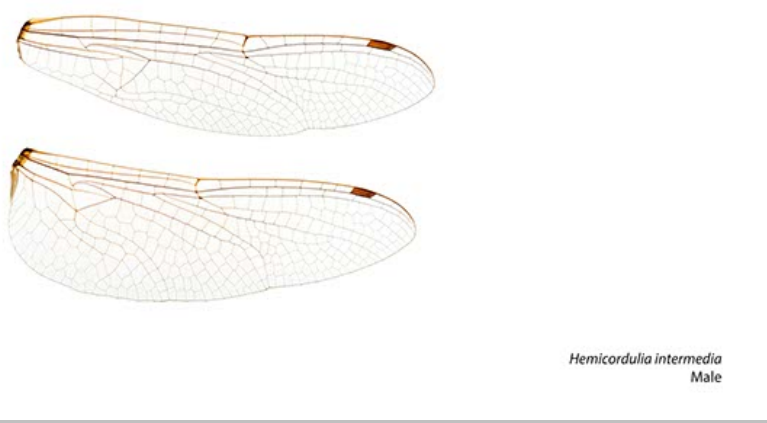

Hemicordulia intermedia male

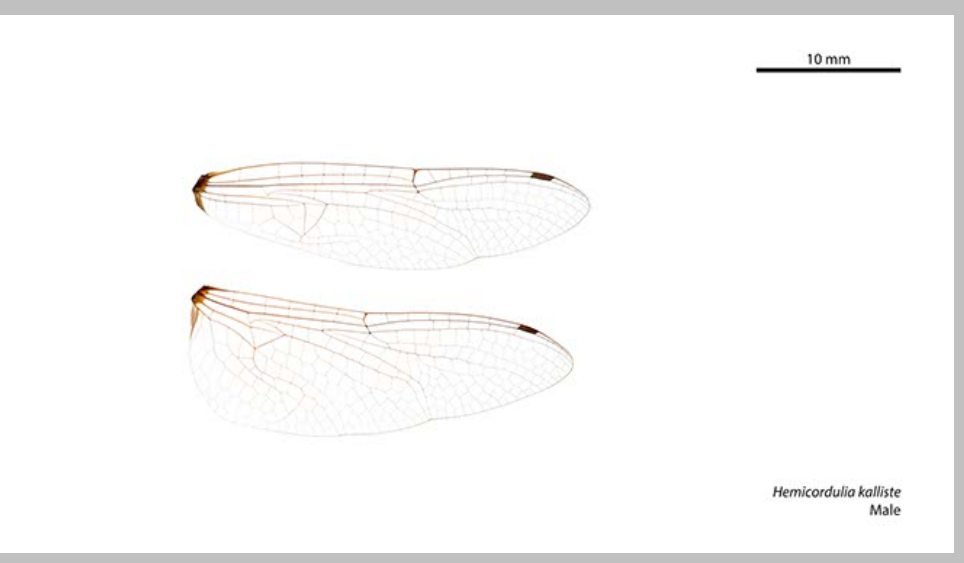

Hemicordulia kalliste male

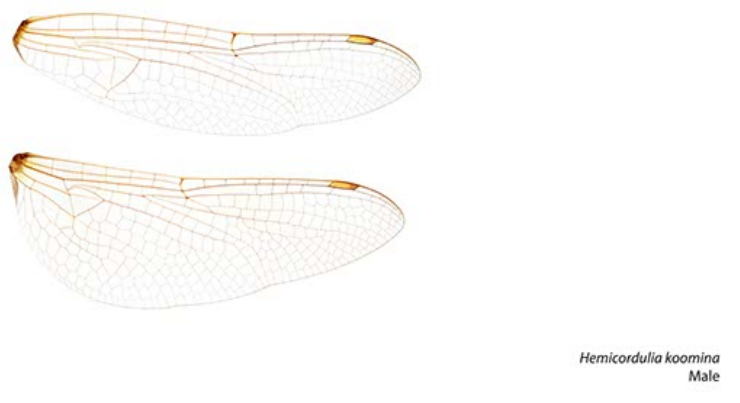

Hemicordulia koomina male 

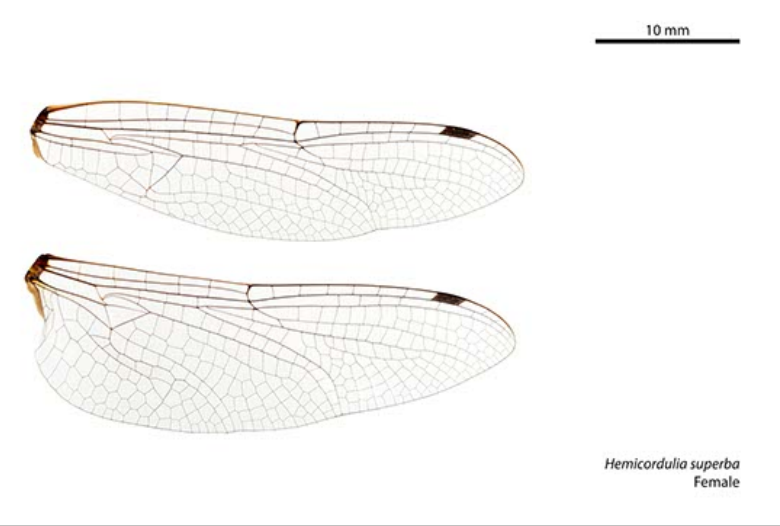

Hemicordulia superba female

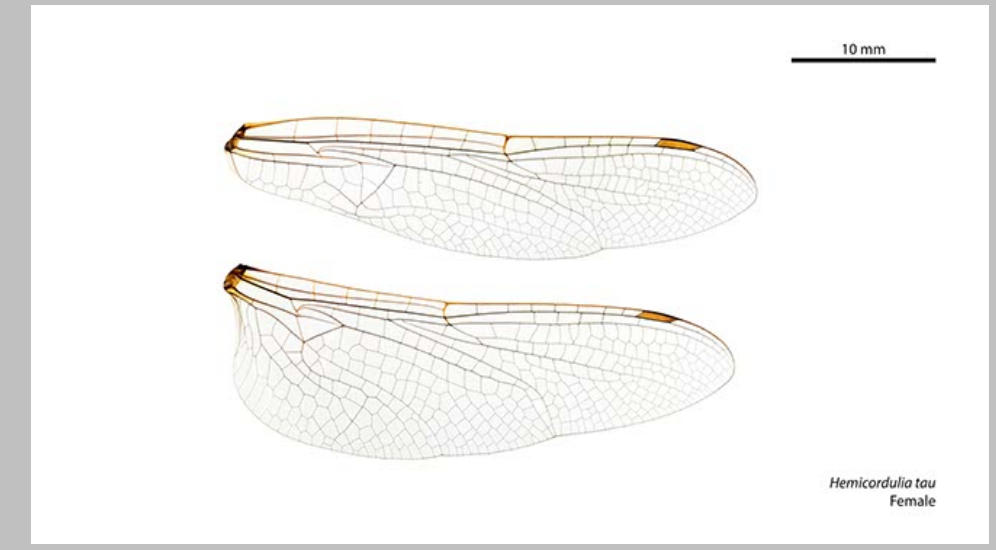

Hemicordulia tau female

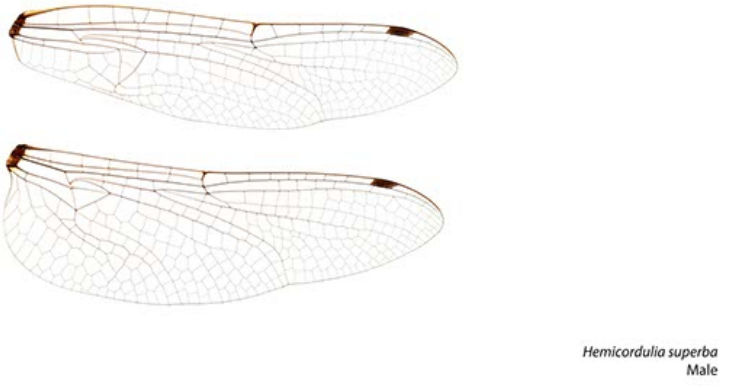

Hemicordulia superba male

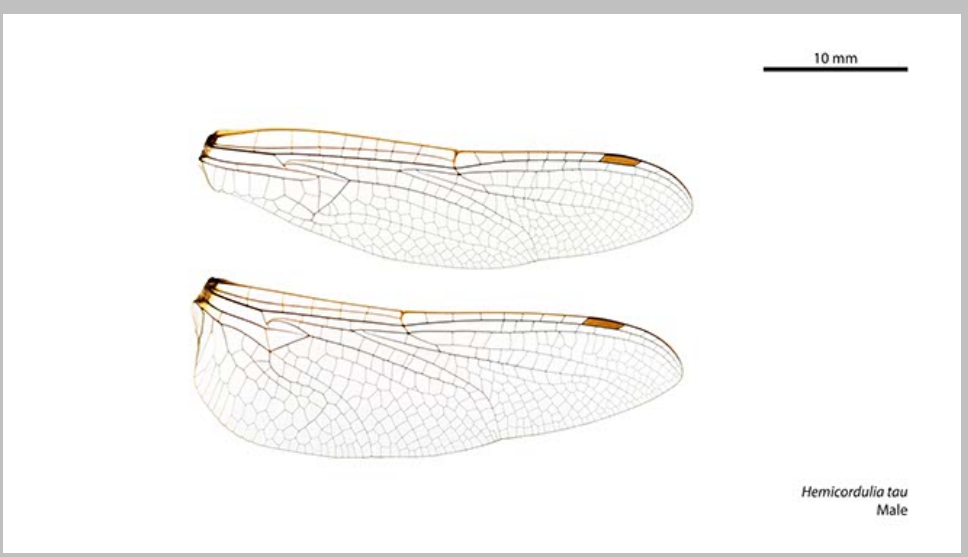

Hemicordulia tau male
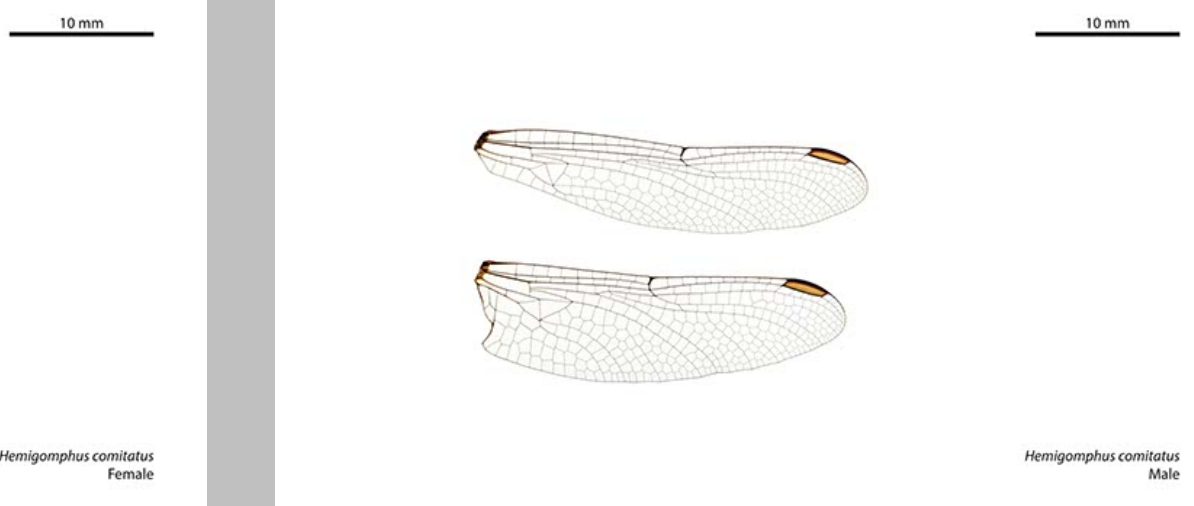


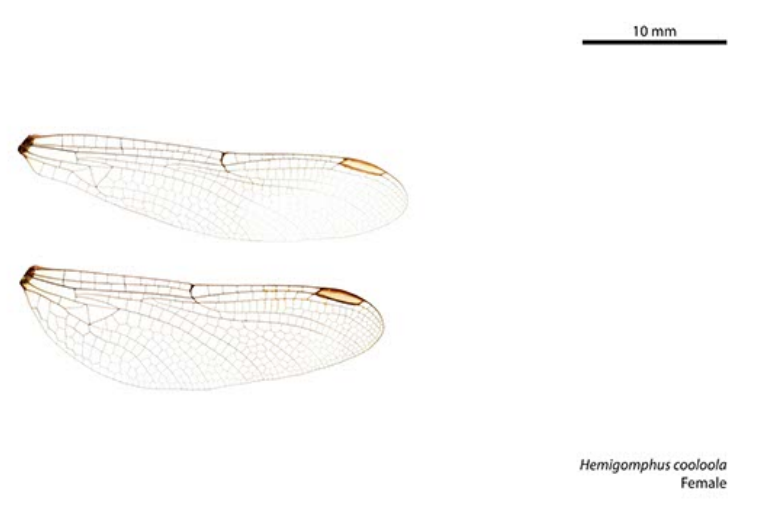

Hemigomphus cooloola female

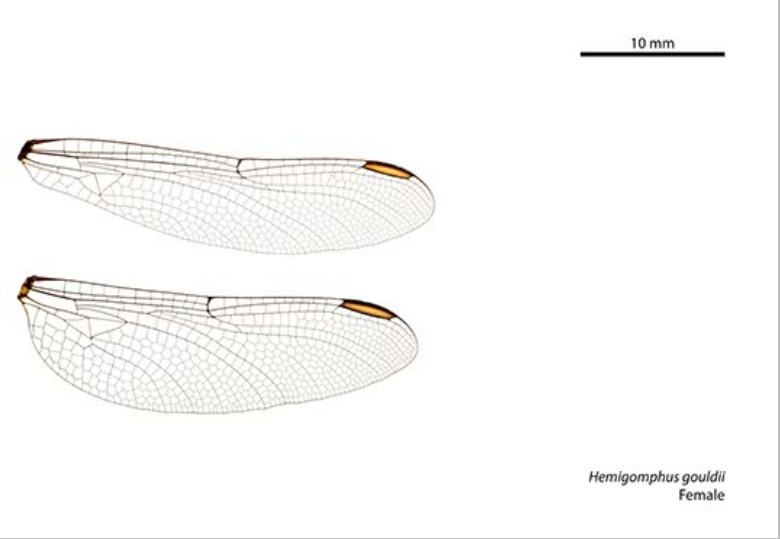

Hemigomphus gouldii female

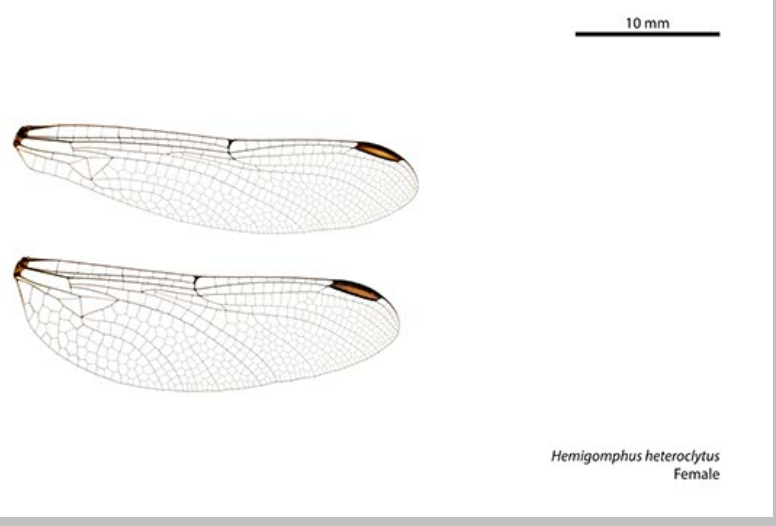

Hemigomphus heteroclytus female

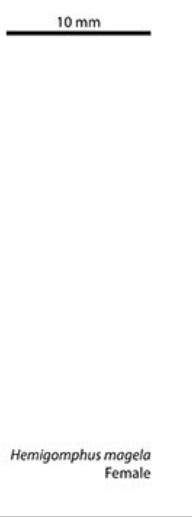



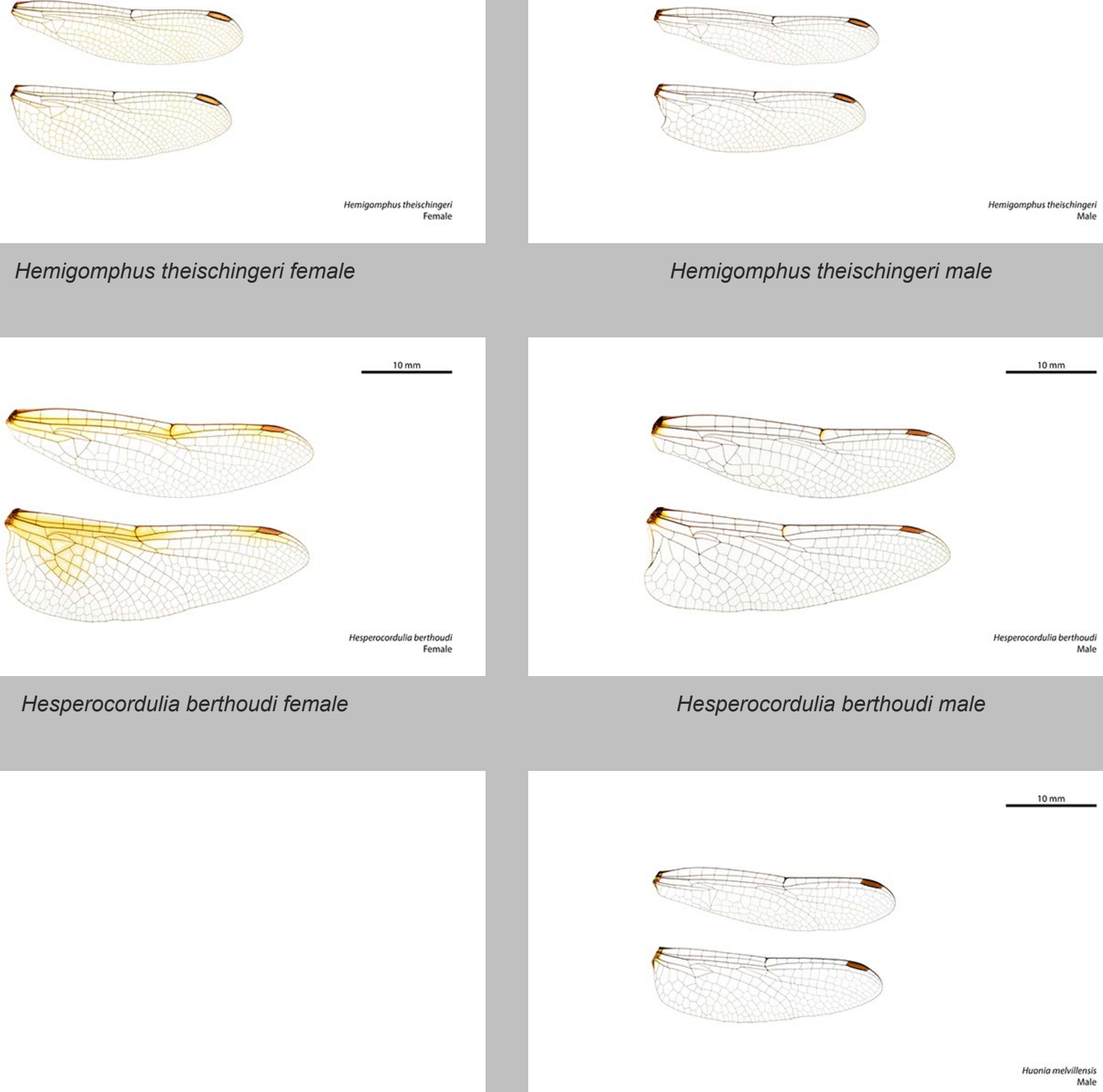

Huonia melvillensis female

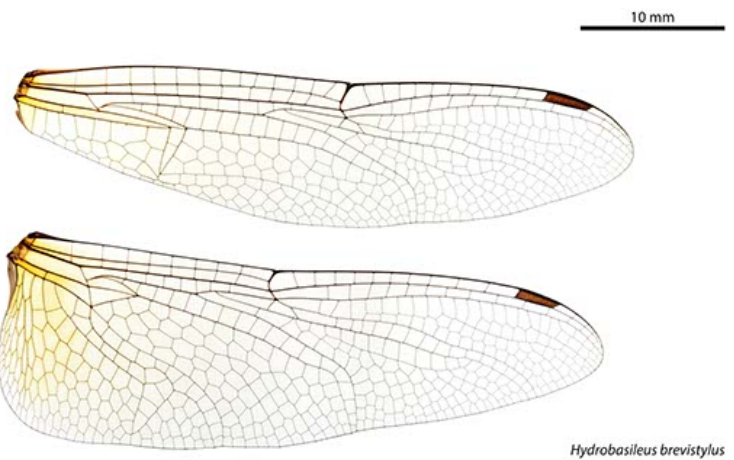

Hydrobasileus brevistylus
Huonia melvillensis male

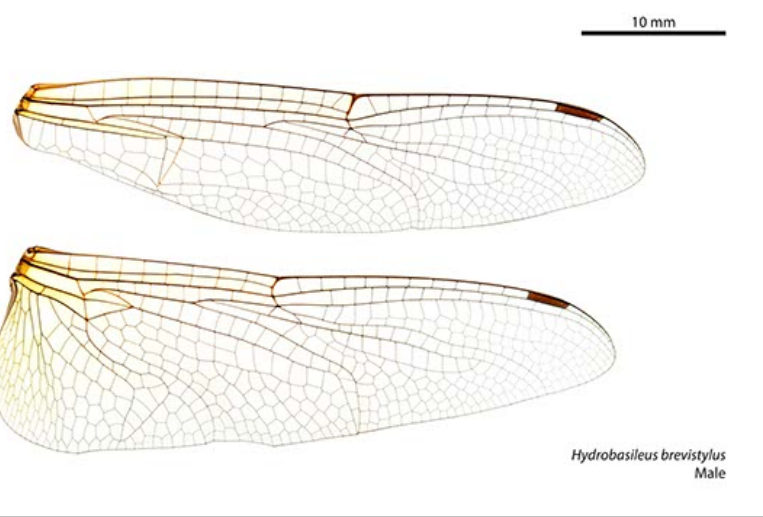

Hydrobasileus brevistylus male 


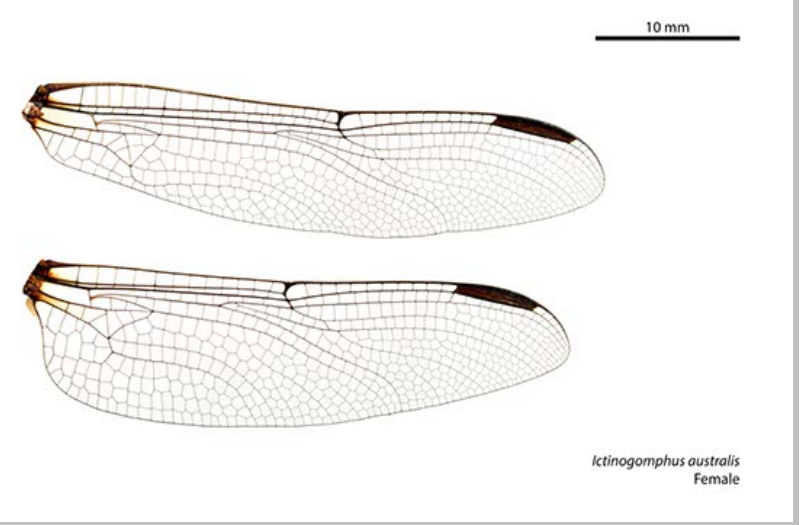

Ictinogomphus australis female

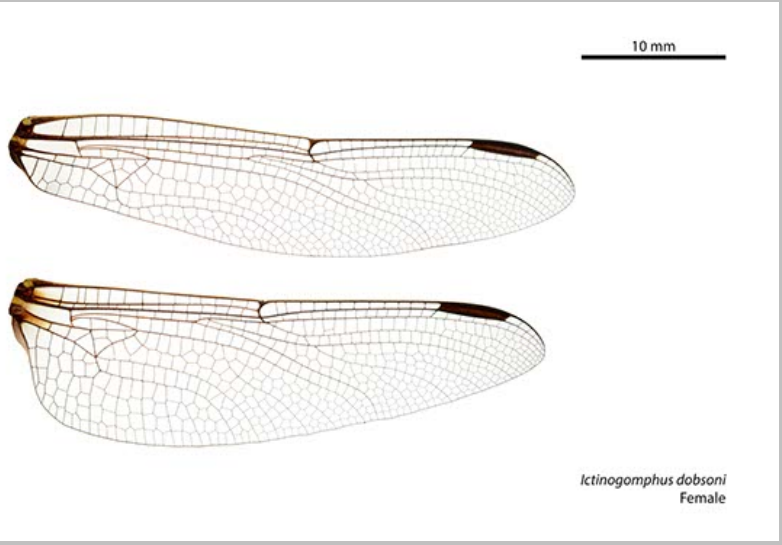

Ictinogomphus dobsoni female

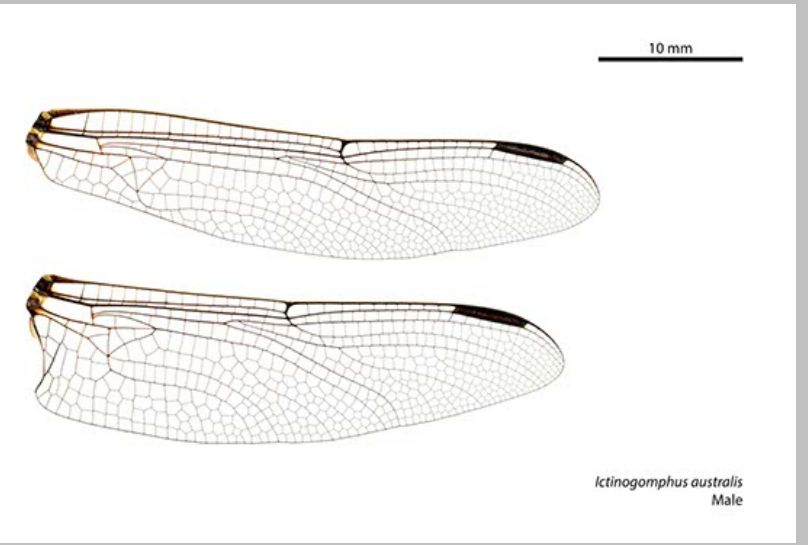

Ictinogomphus australis male

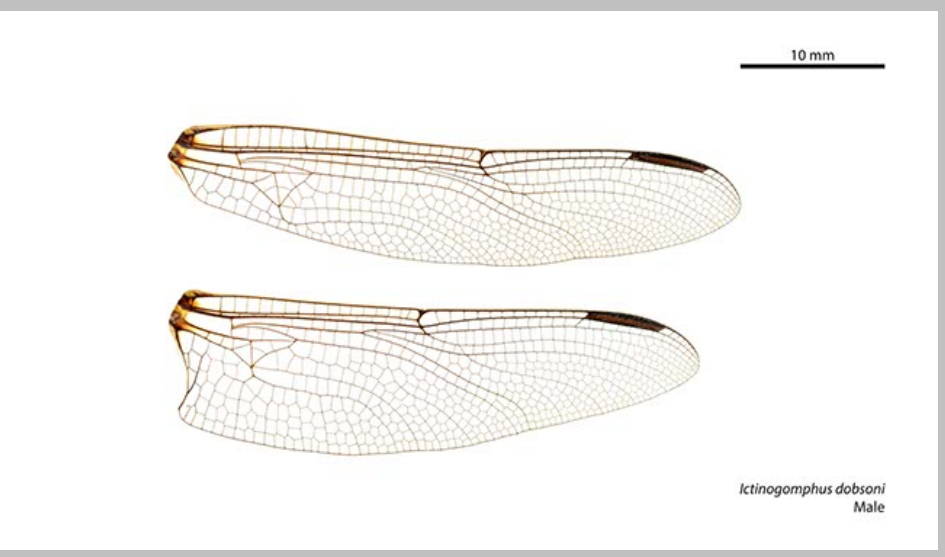

Ictinogomphus dobsoni male

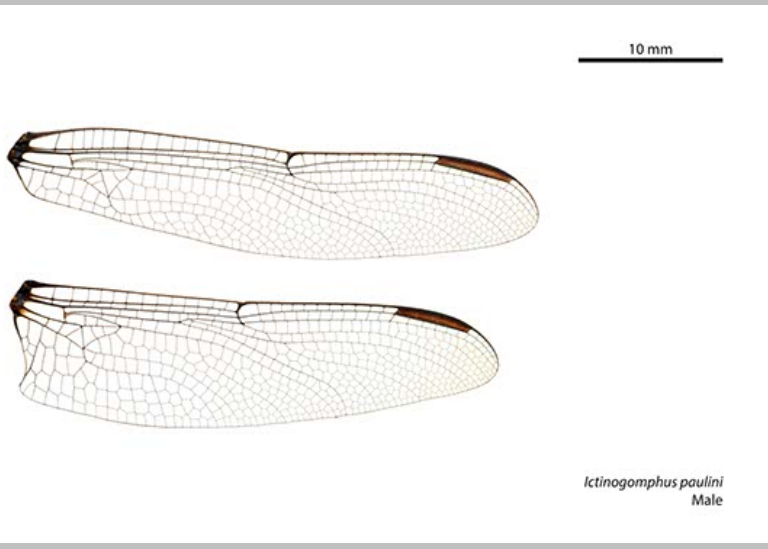

Ictinogomphus paulini male
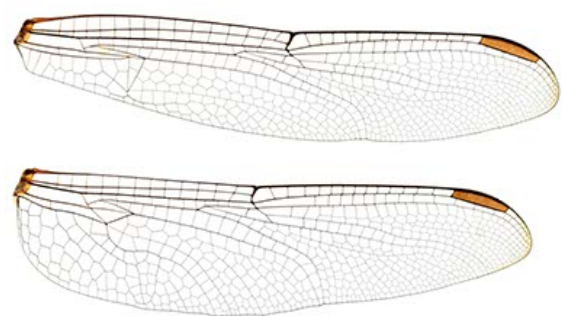

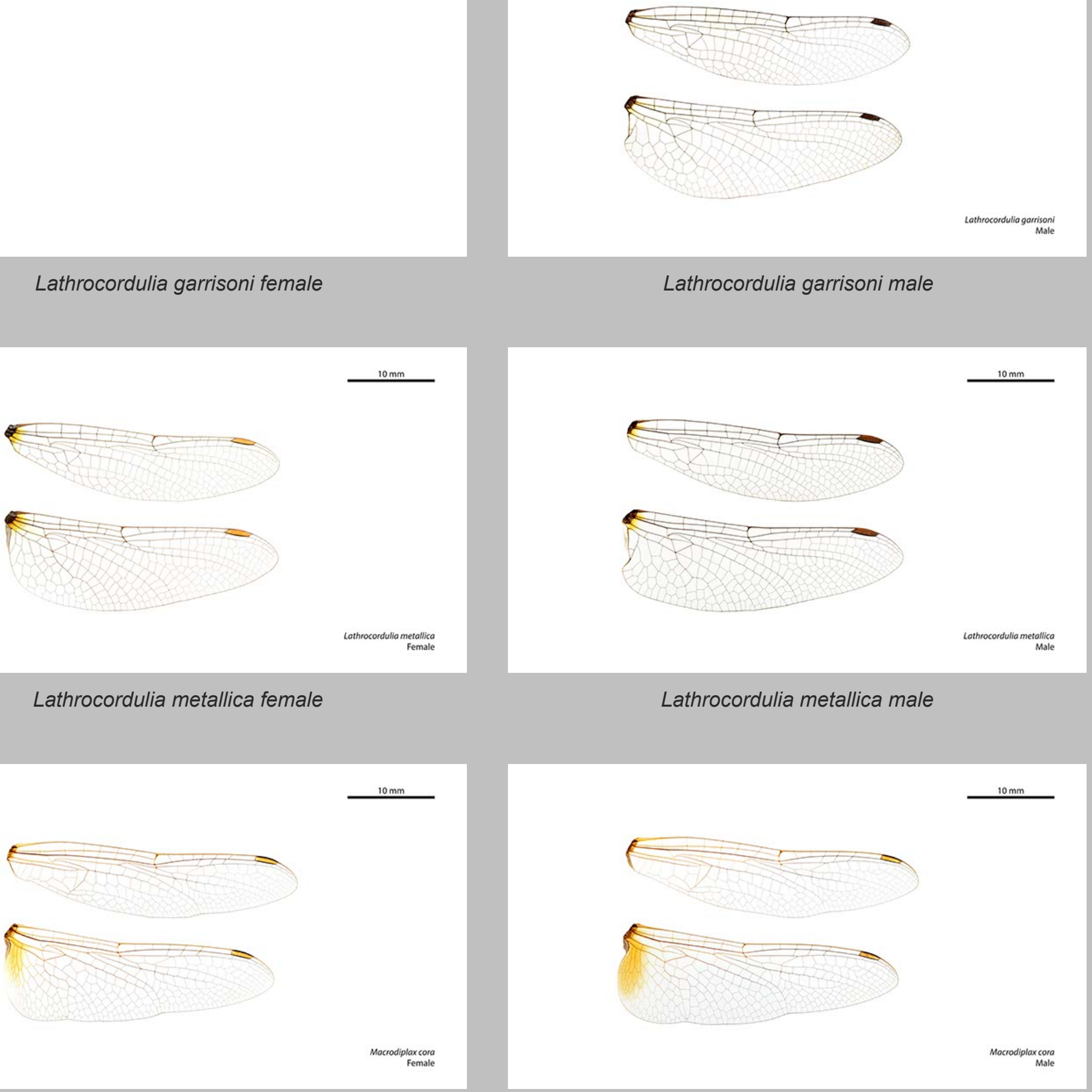

Macrodiplax cora female

Macrodiplax cora male
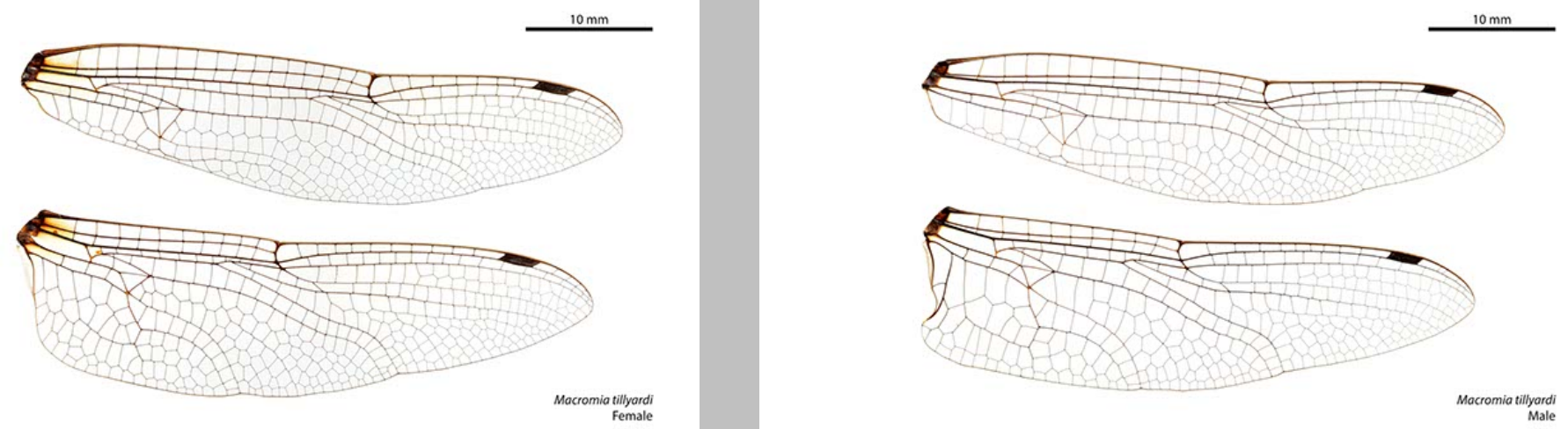


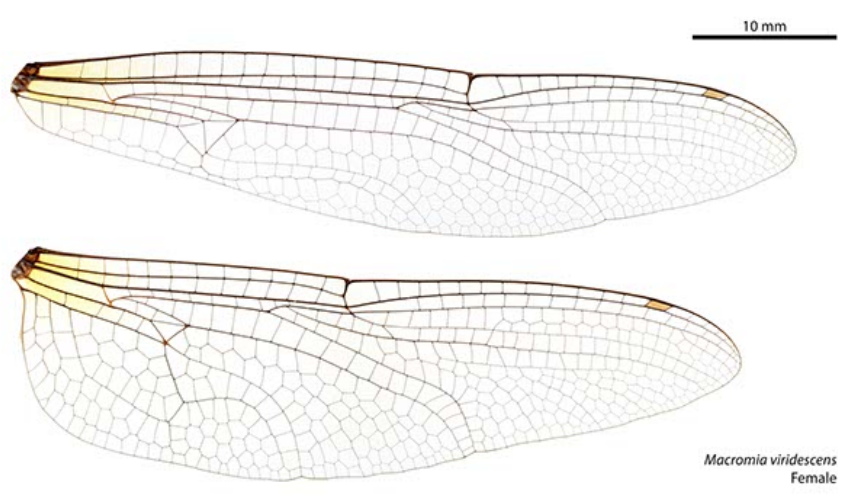

Macromia viridescens female

Metaphya tillyardi female
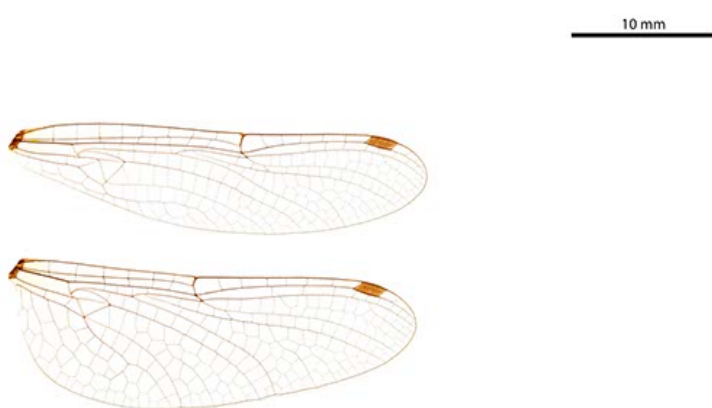

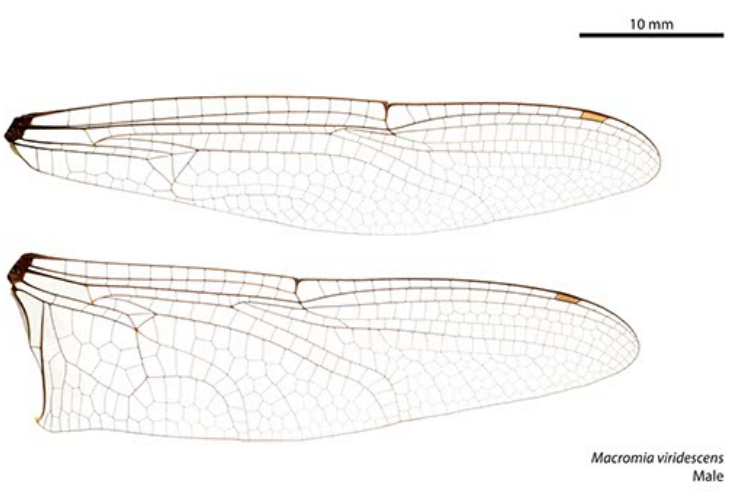

Macromia viridescens male
Metaphya tillyardi male

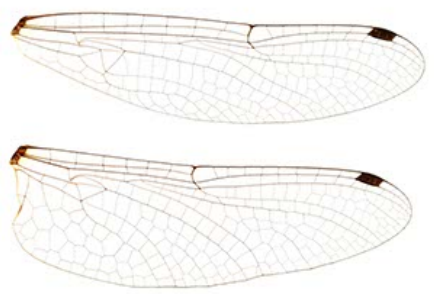

Micromidia atrifrons female

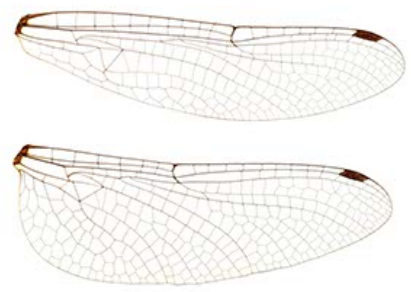

Micromidia convergens
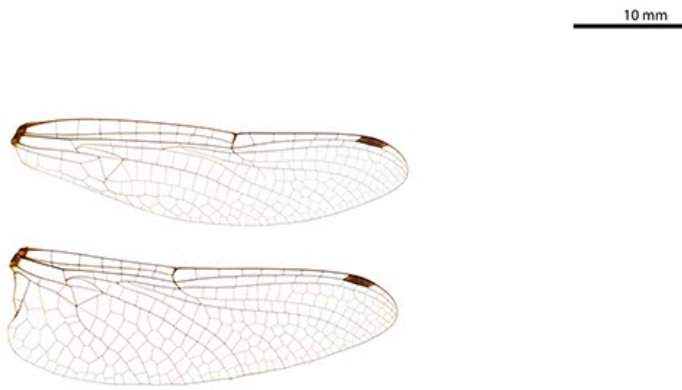

Micromidia convergens 


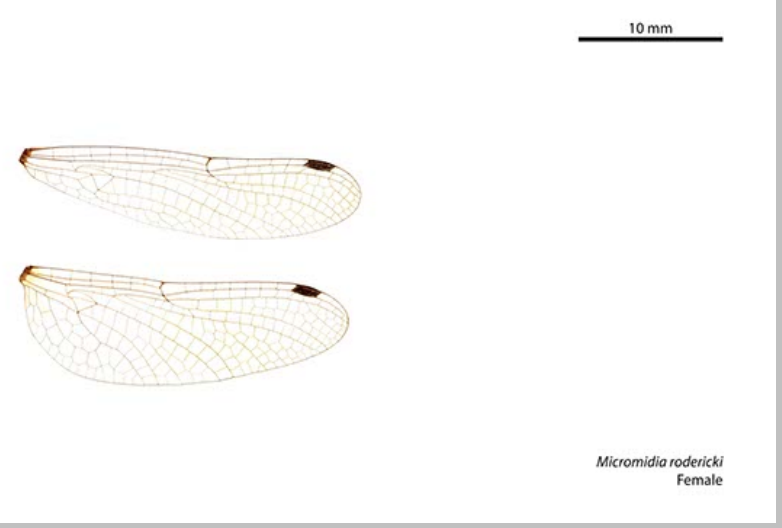

Micromidia rodericki female

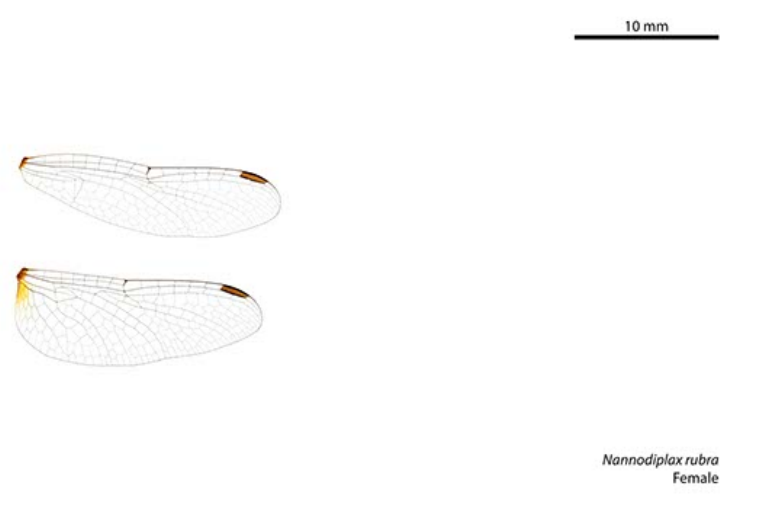

Nannodiplax rubra female

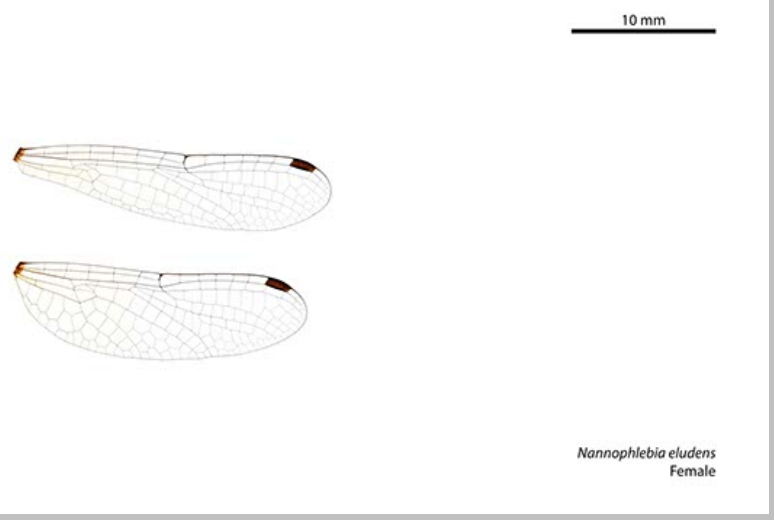

Nannophlebia eludens female

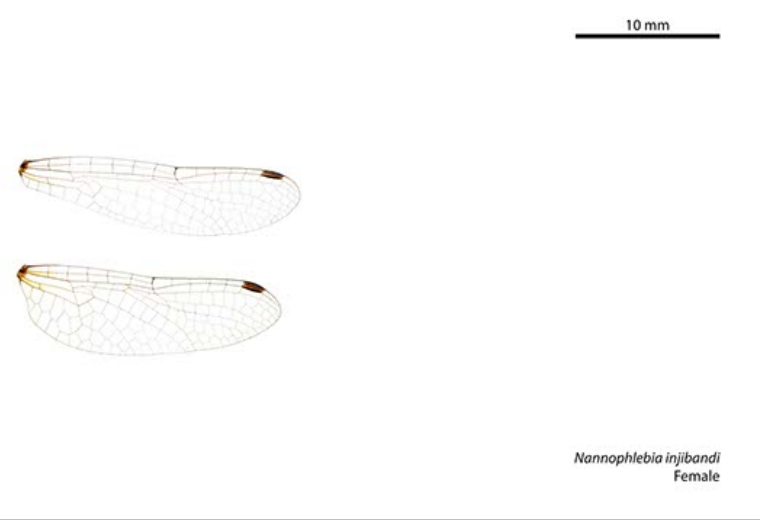

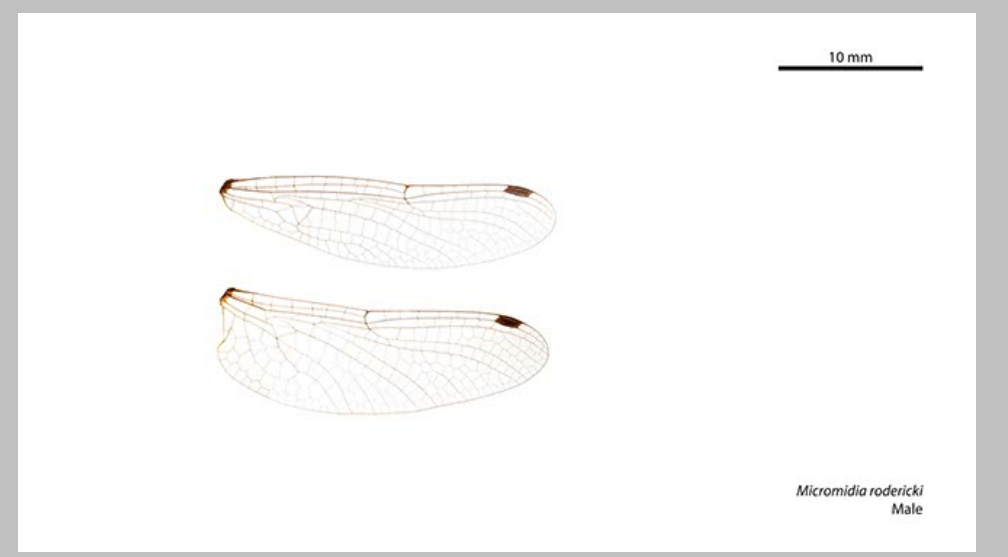

Micromidia rodericki male

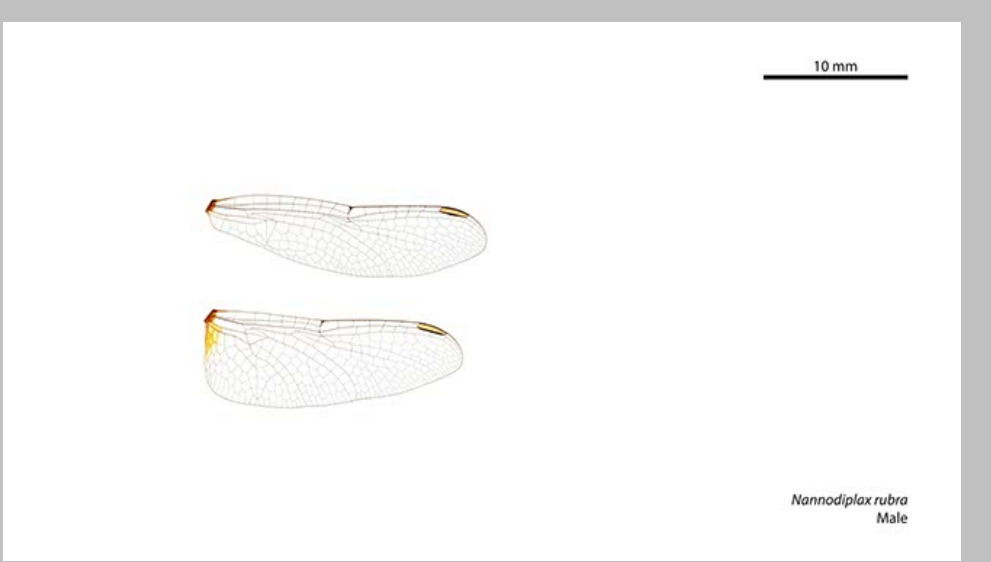

Nannodiplax rubra male

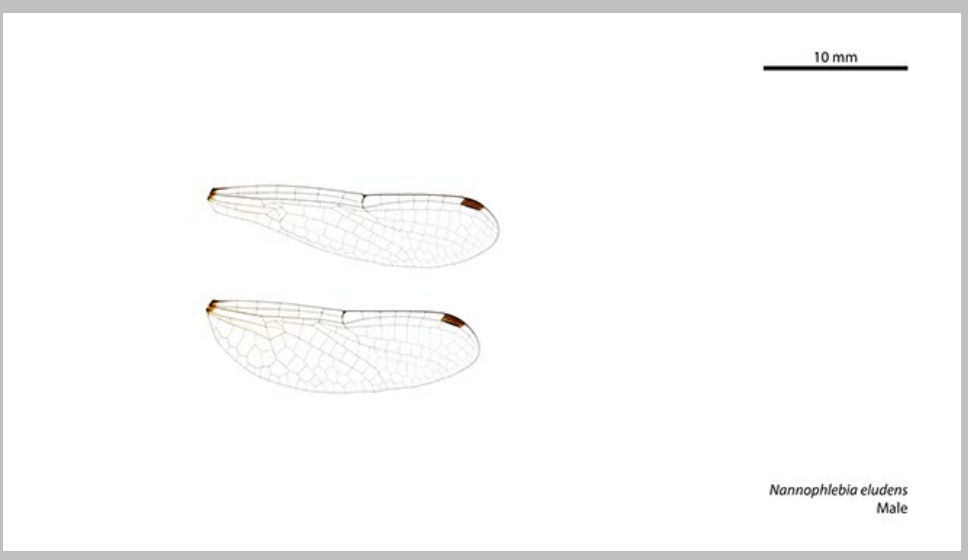

Nannophlebia eludens male

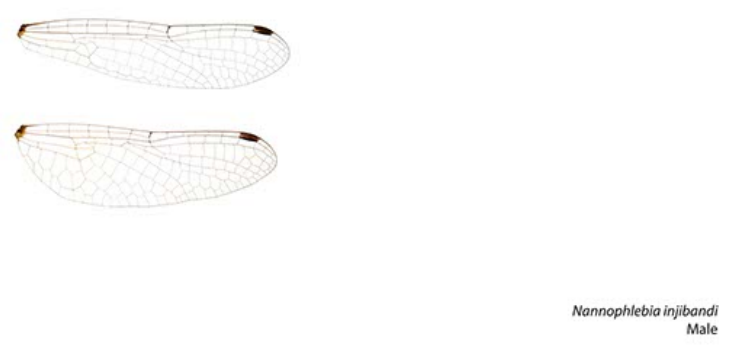

Nannophlebia injibandi male 


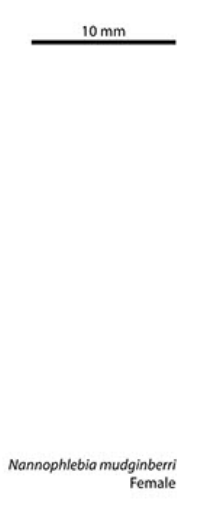

Nannophlebia mudginberri female

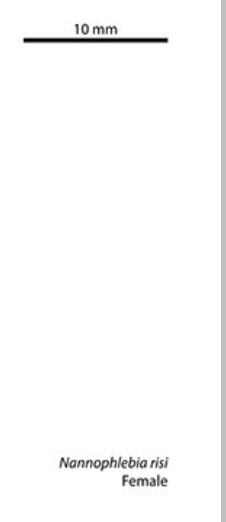

Nannophlebia risi female

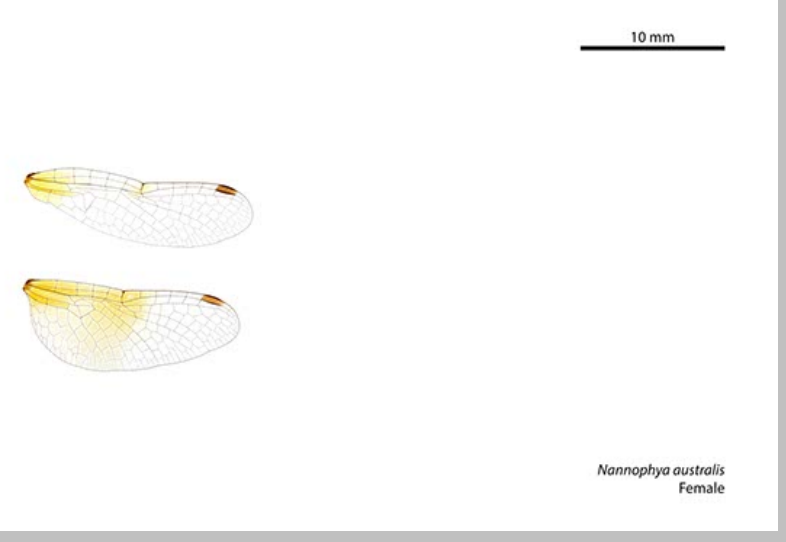

Nannophya australis female

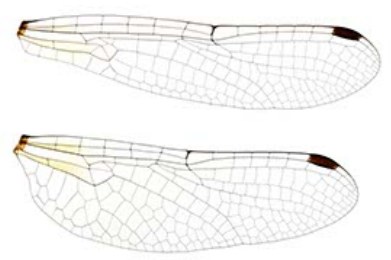

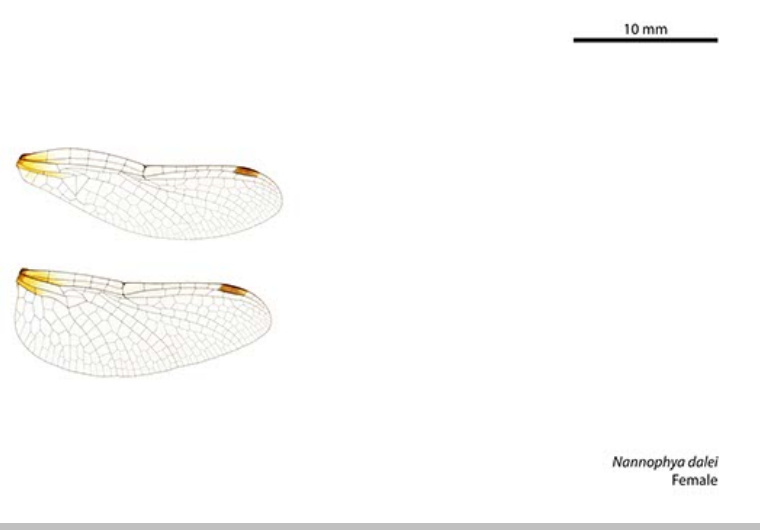
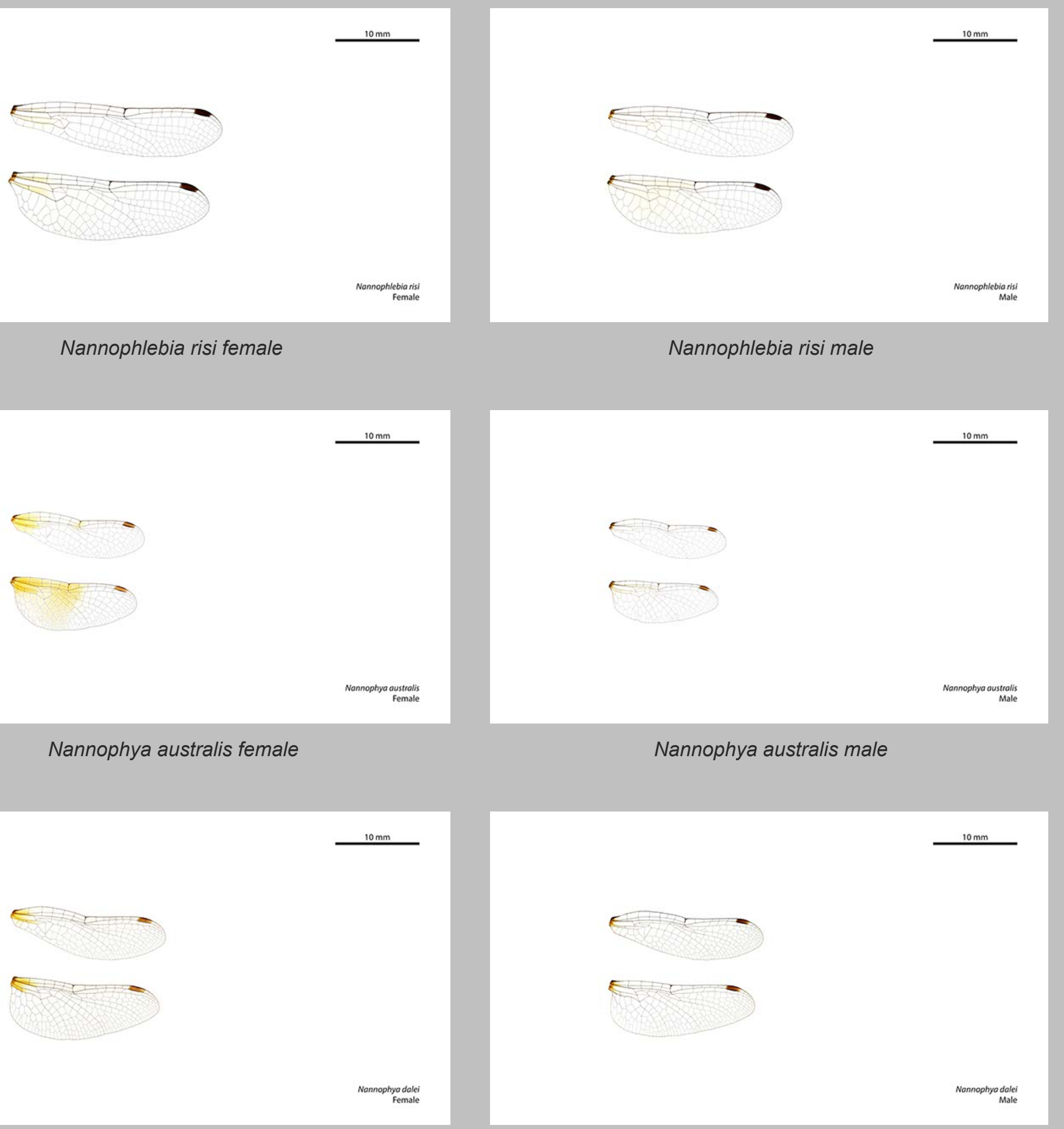

Nannophlebia risi male

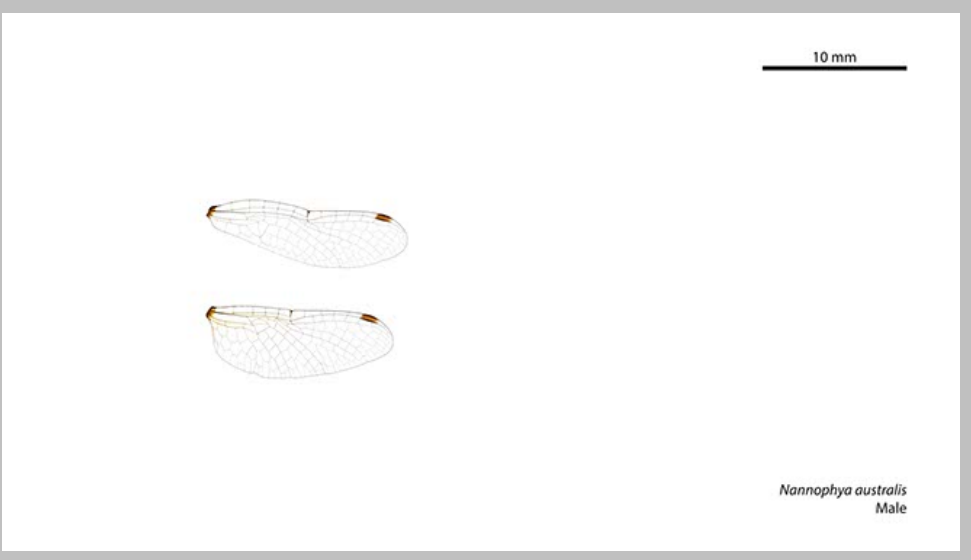

Nannophya australis male 


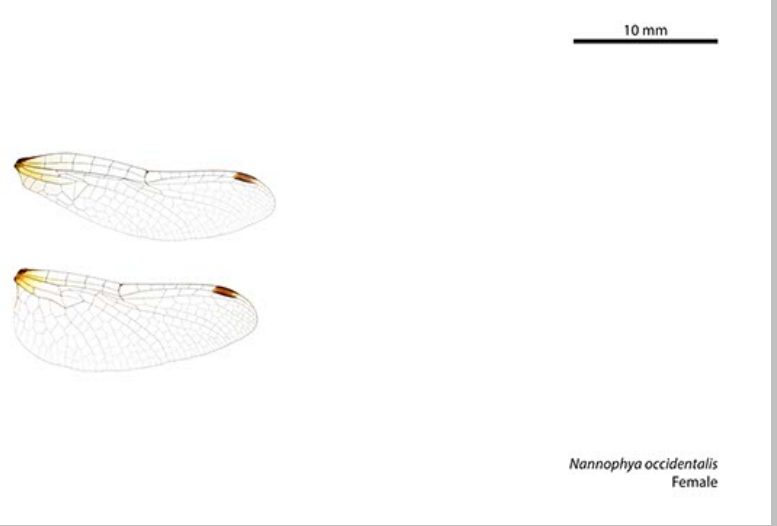

Nannophya occidentalis female

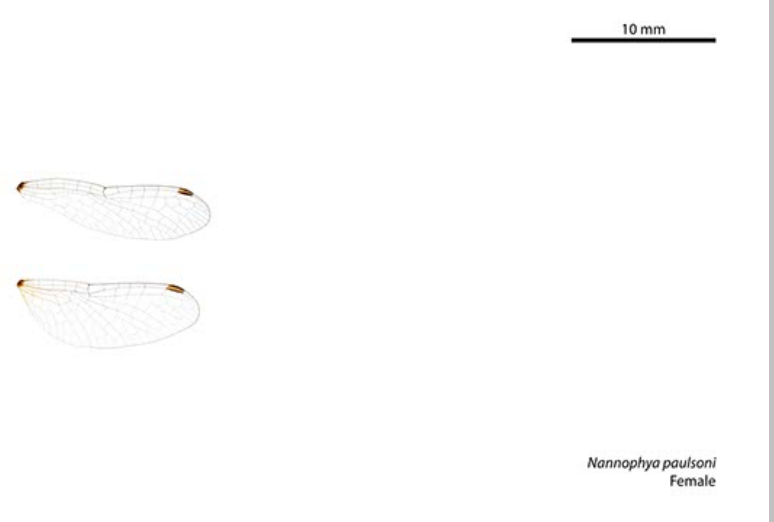

Nannophya paulsoni female
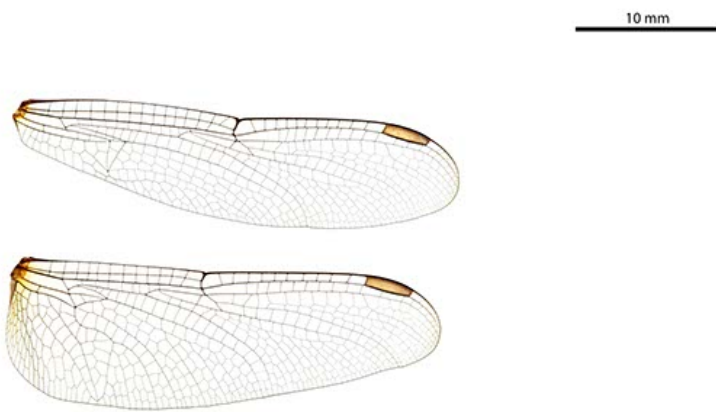

Neurothemis olligoneura

Neurothemis oligoneura female

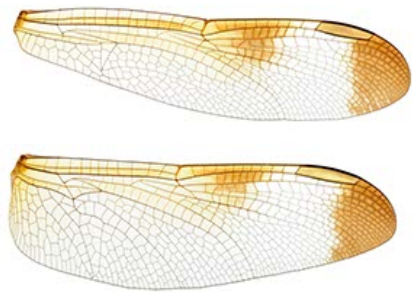

Neurothemis stigmatizans
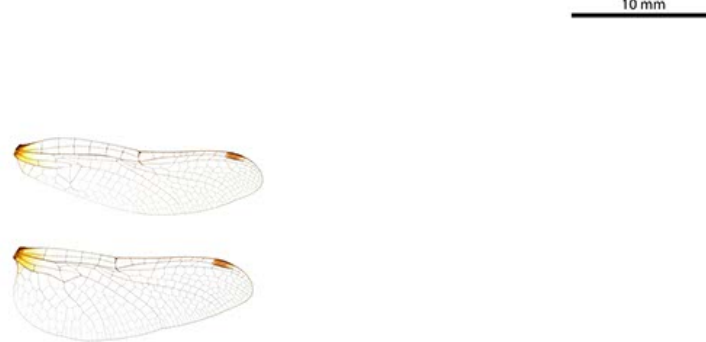

Nannophya occidentalis male

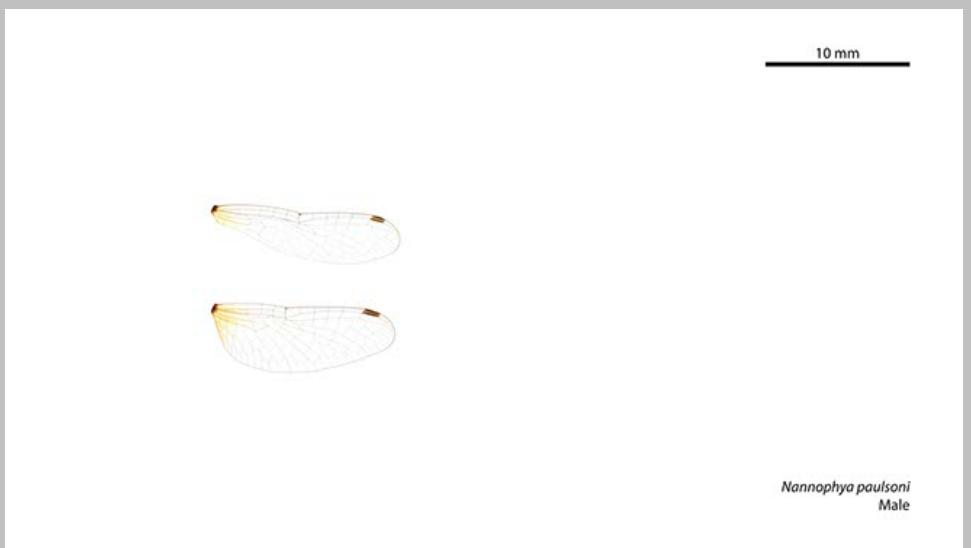

Nannophya paulsoni male

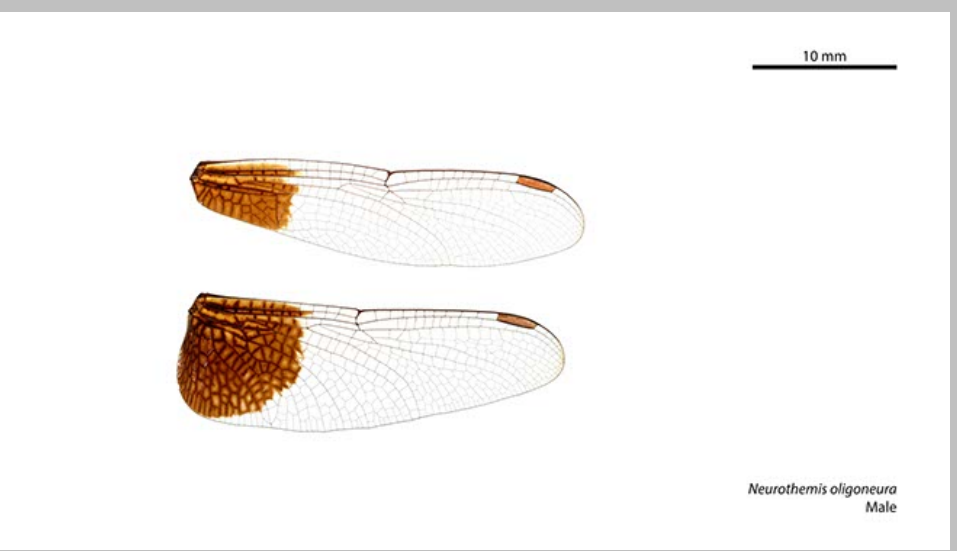

Neurothemis oligoneura male

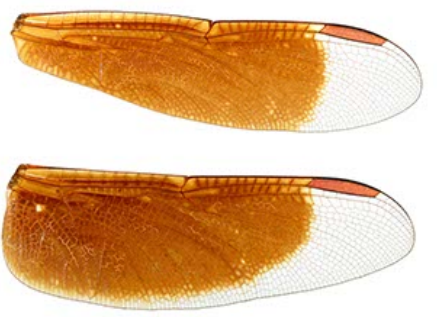

Neurothemis stigmatizan

Neurothemis stigmatizans male 


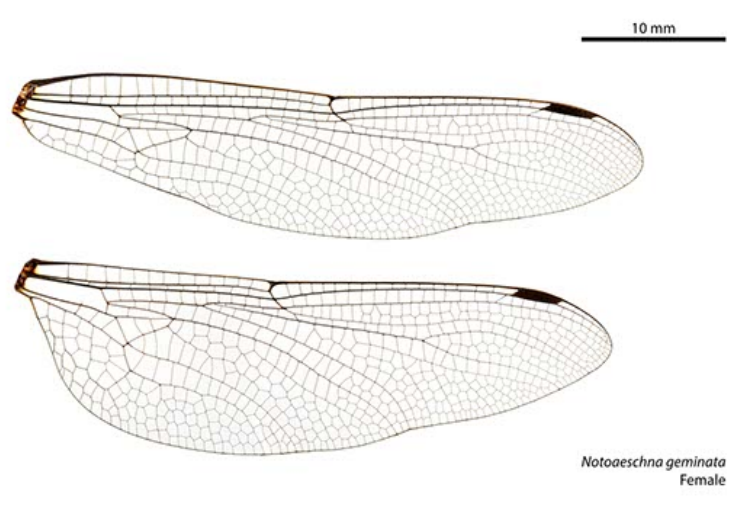

Notoaeschna geminata female

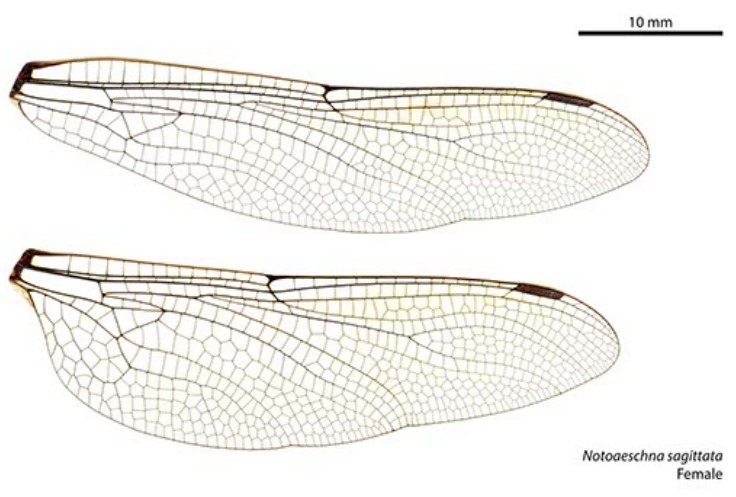

Notoaeschna sagittata female

Notolibellula bicolor female
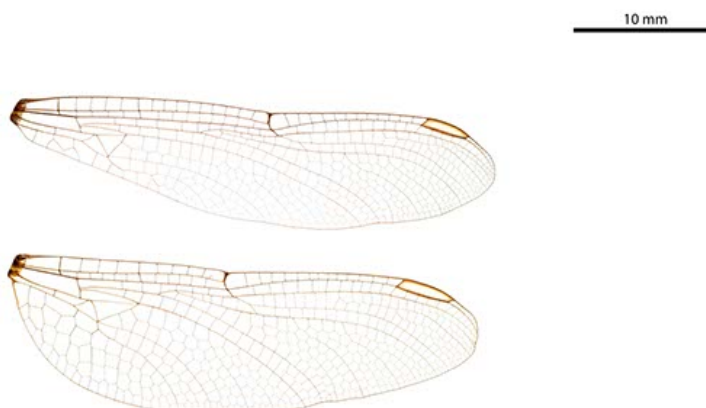

Odontogomphus donnellyi

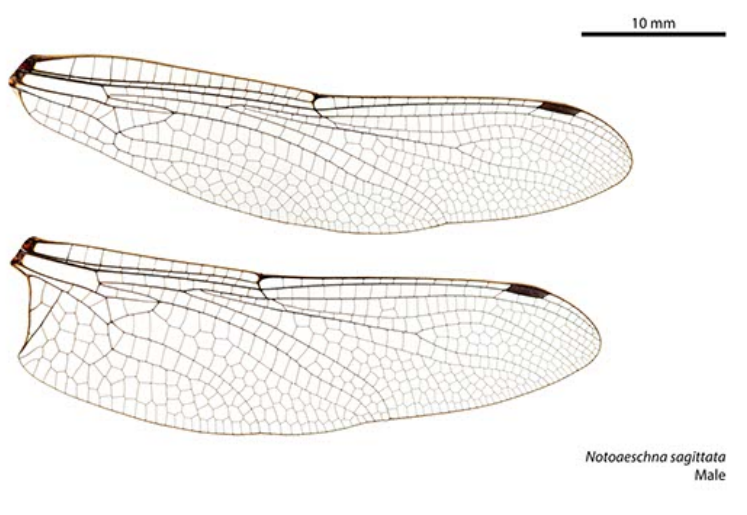

Notoaeschna sagittata male

Notoaeschna geminata male

Notolibellula bicolor male

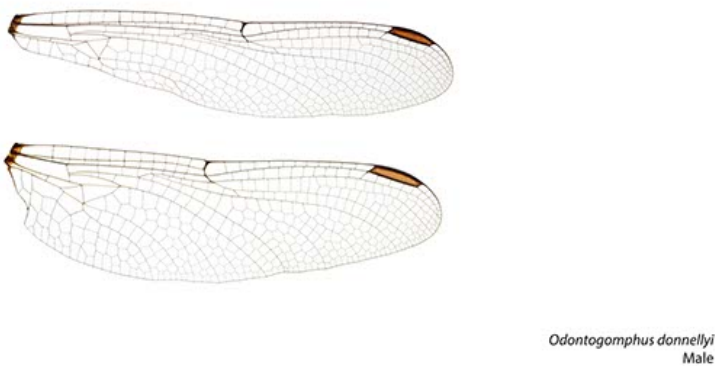




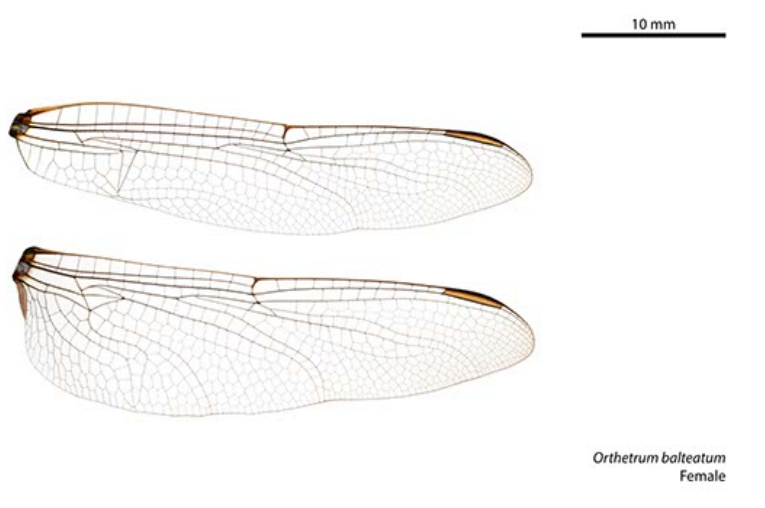

Orthetrum balteatum female
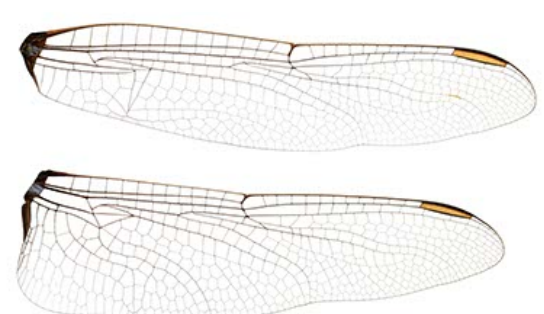

Orthetrum balteatum male

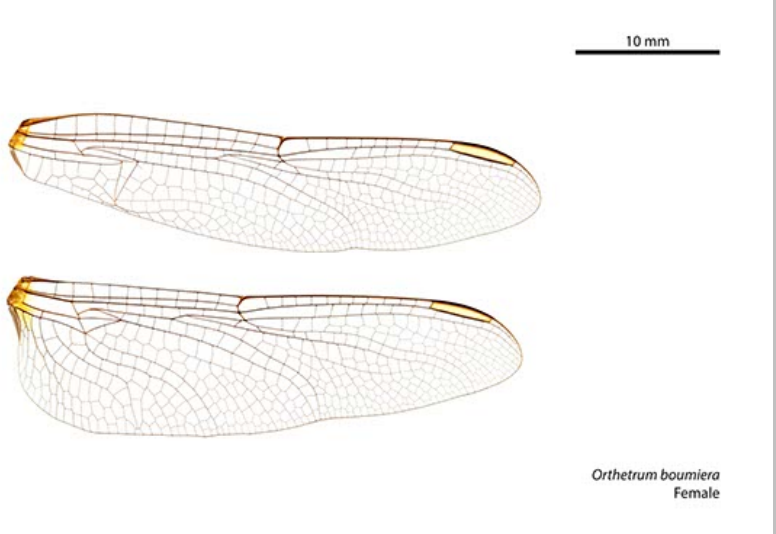

Orthetrum boumiera female

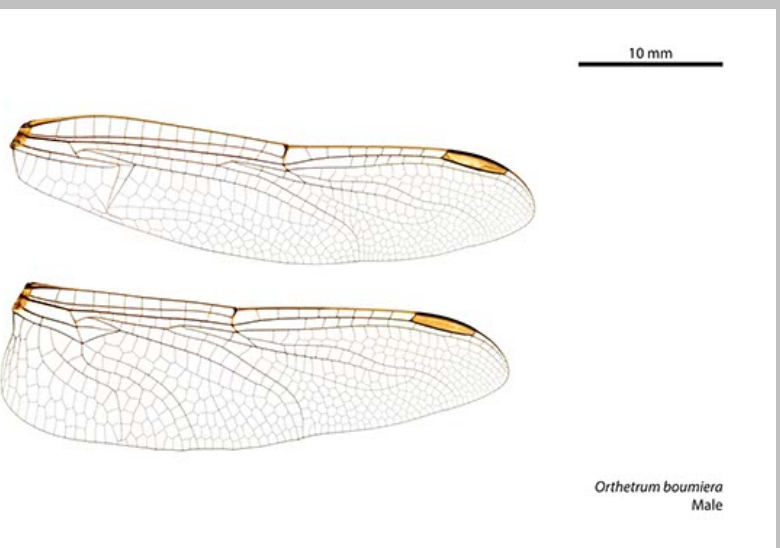

Orthetrum boumiera male

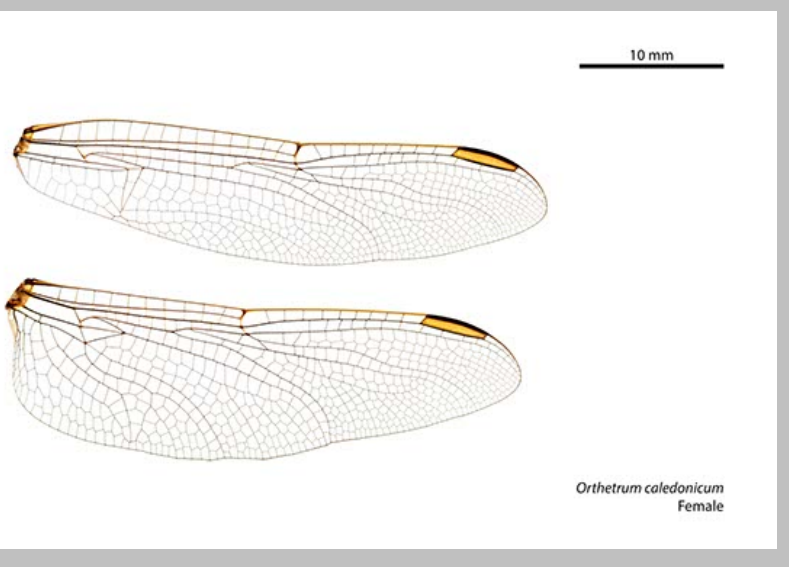

Orthetrum caledonicum female

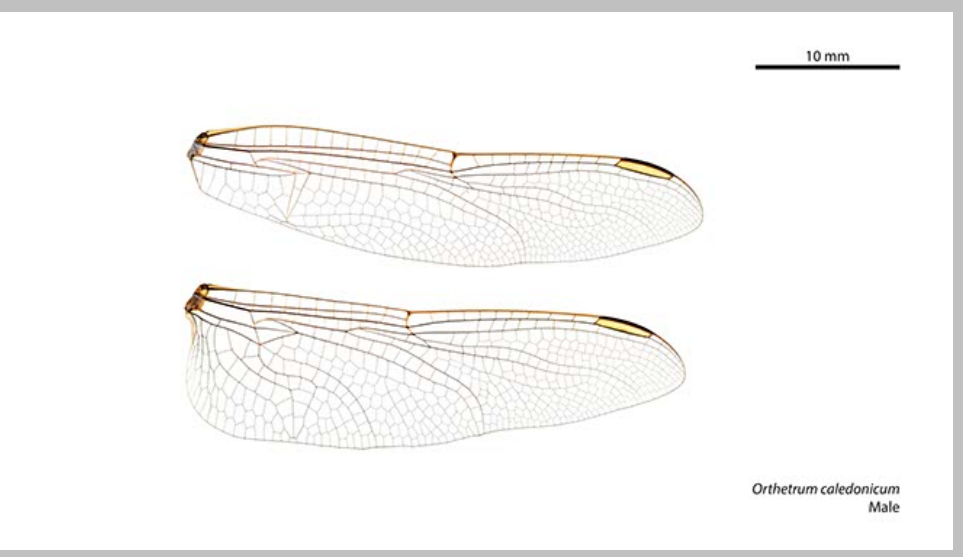

Orthetrum caledonicum male

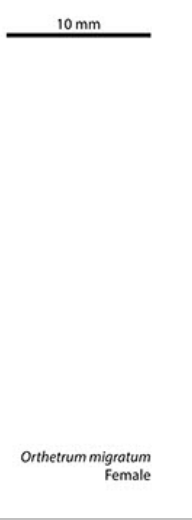



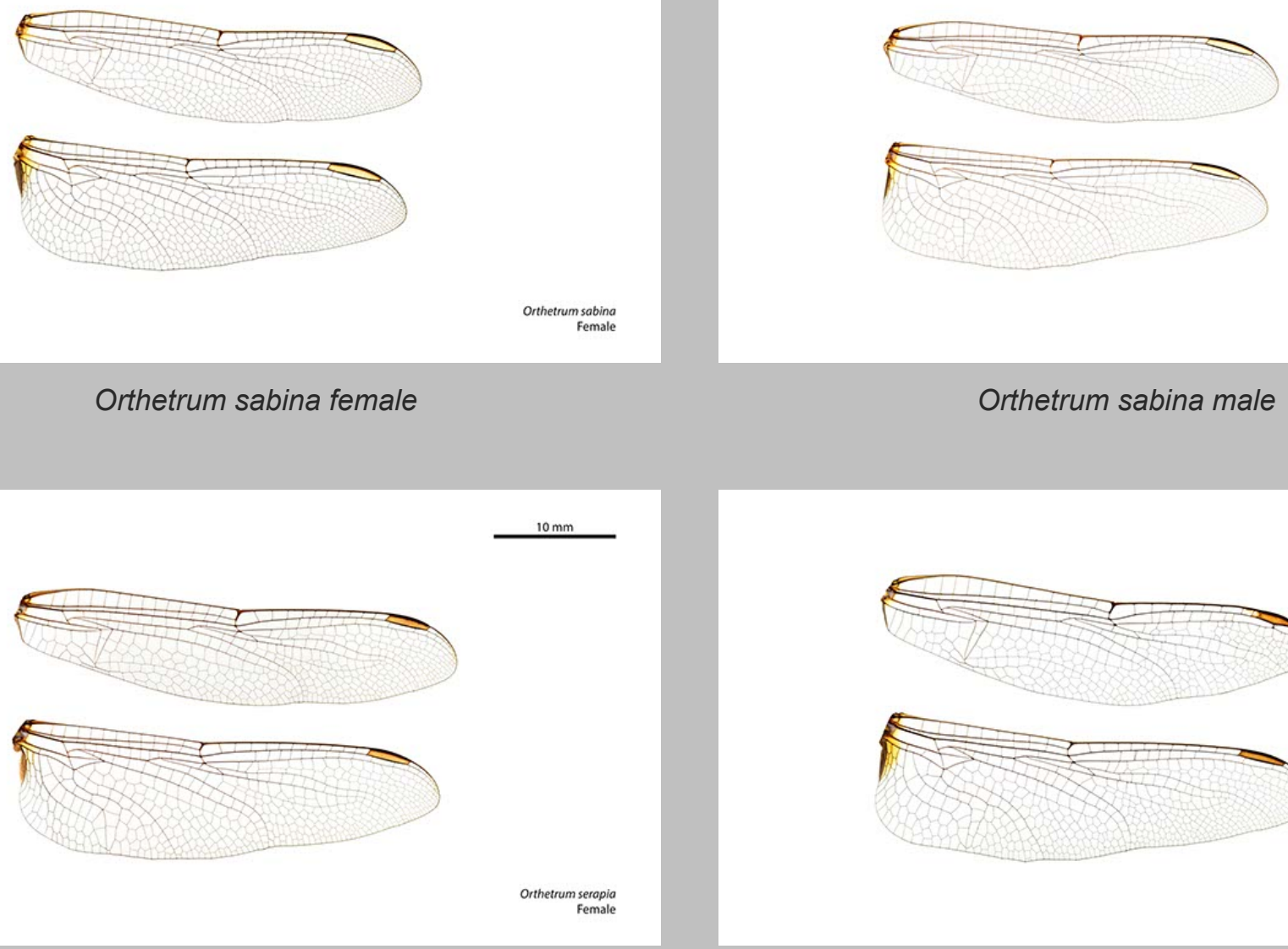

Orthetrum serapia female
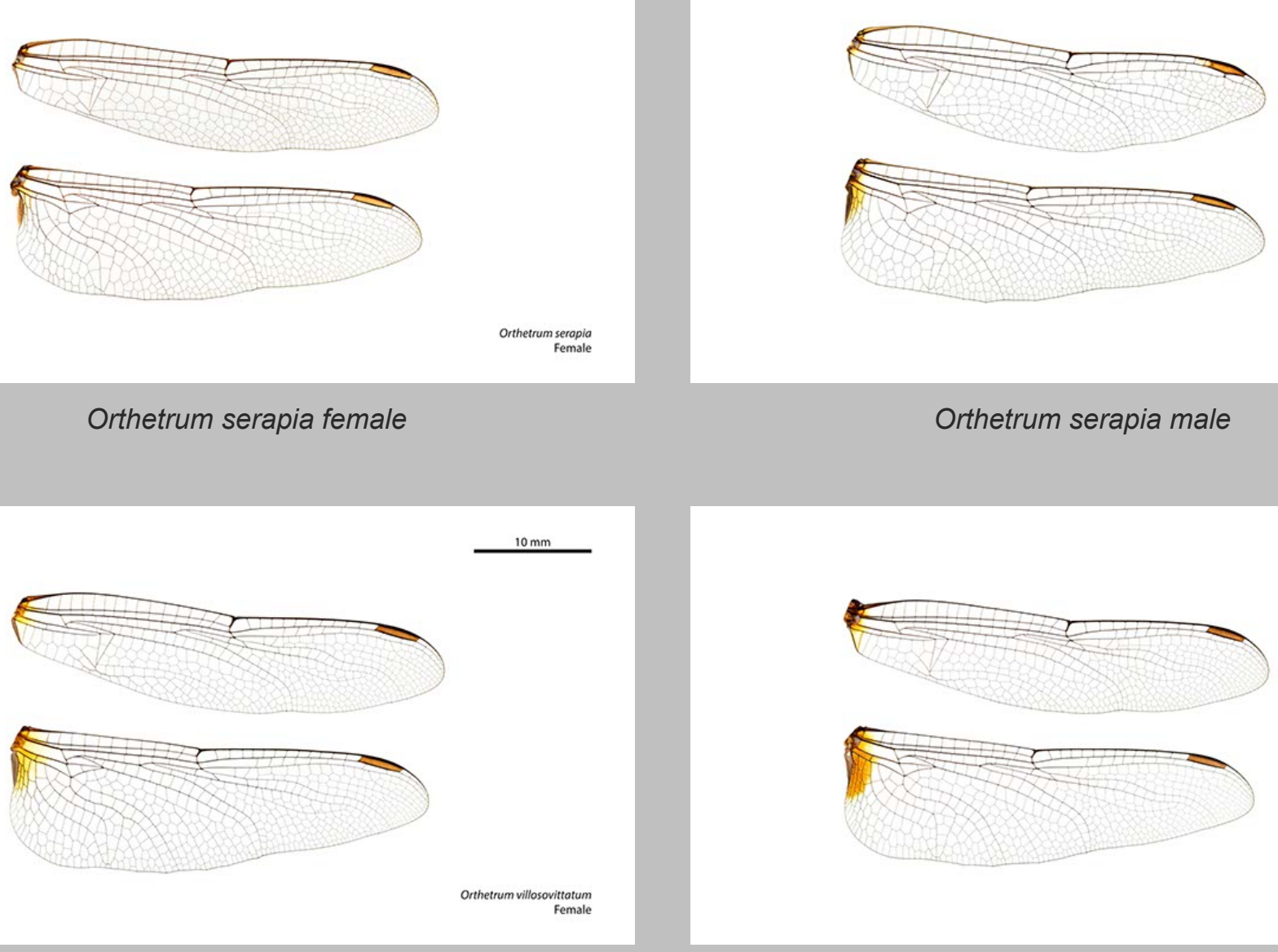

Orthetrum villosovittatum female

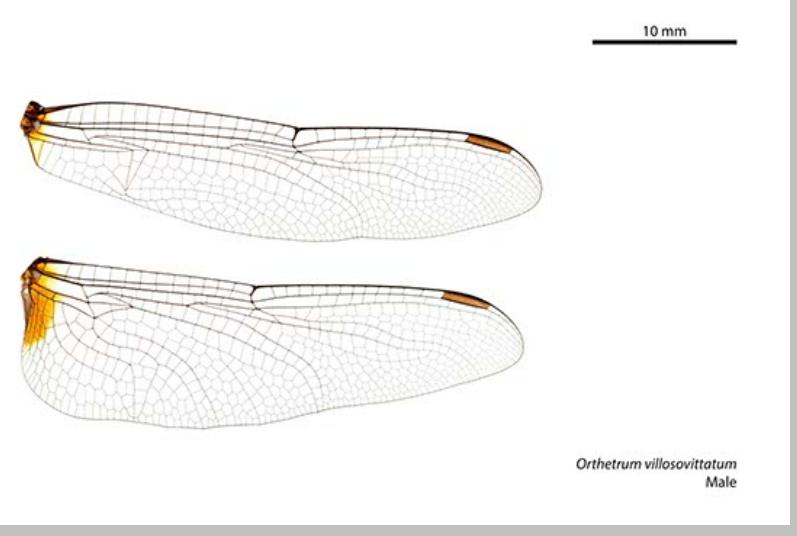

Orthetrum villosovittatum male
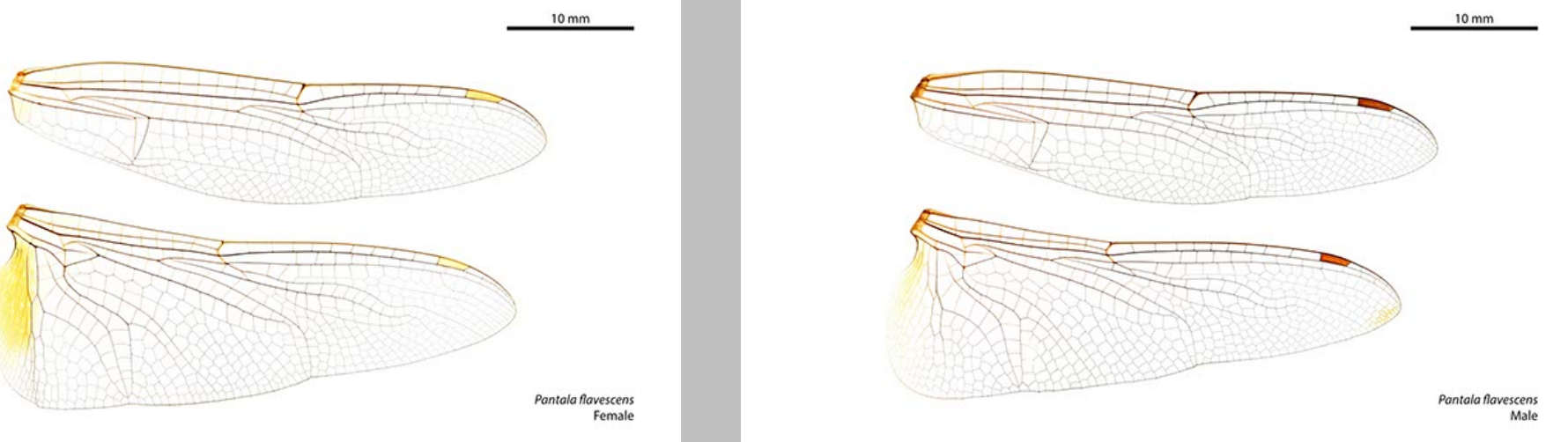


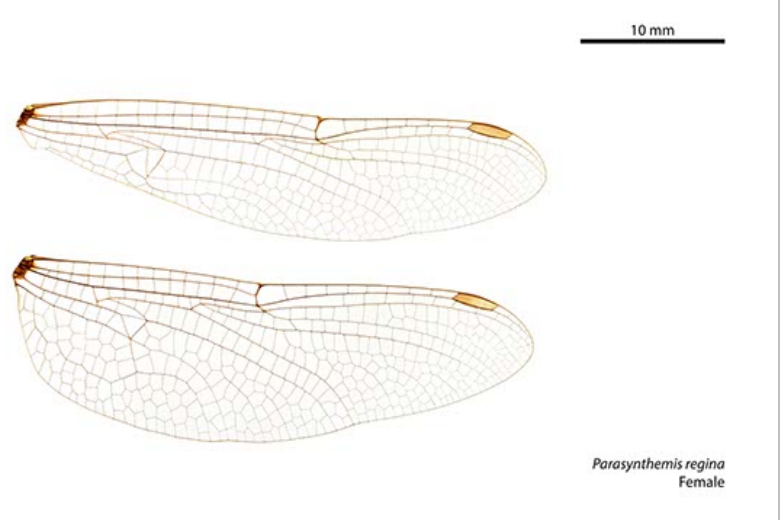

Parasynthemis regina female

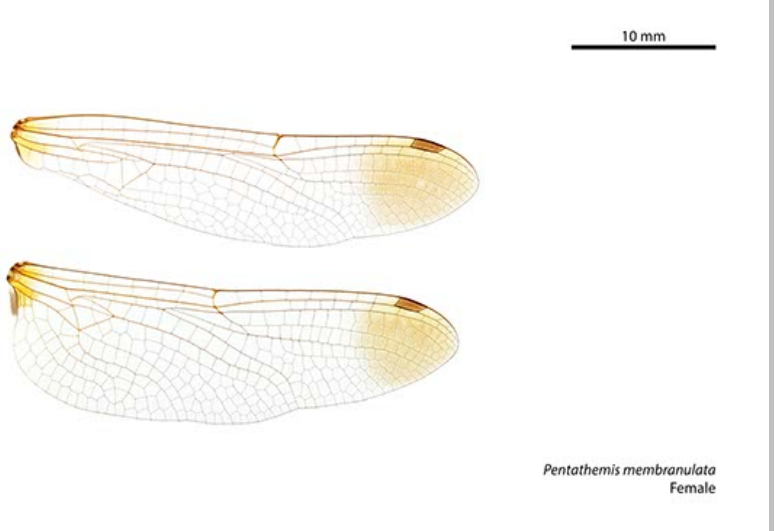

Pentathemis membranulata female

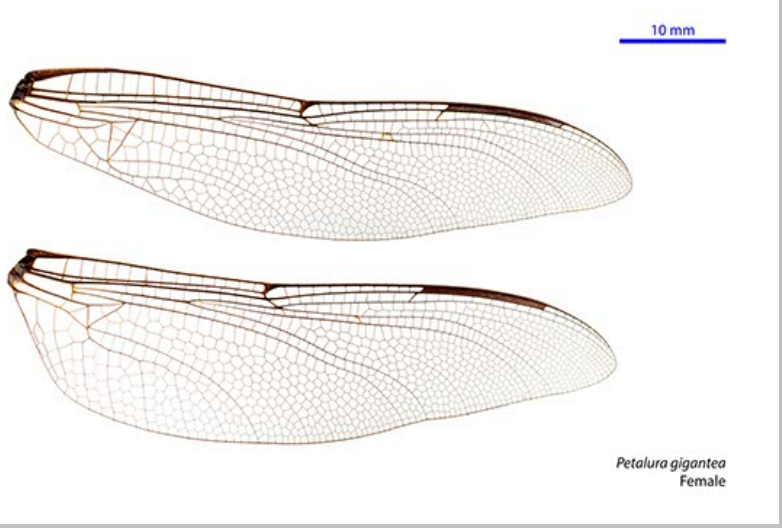

Petalura gigantea female

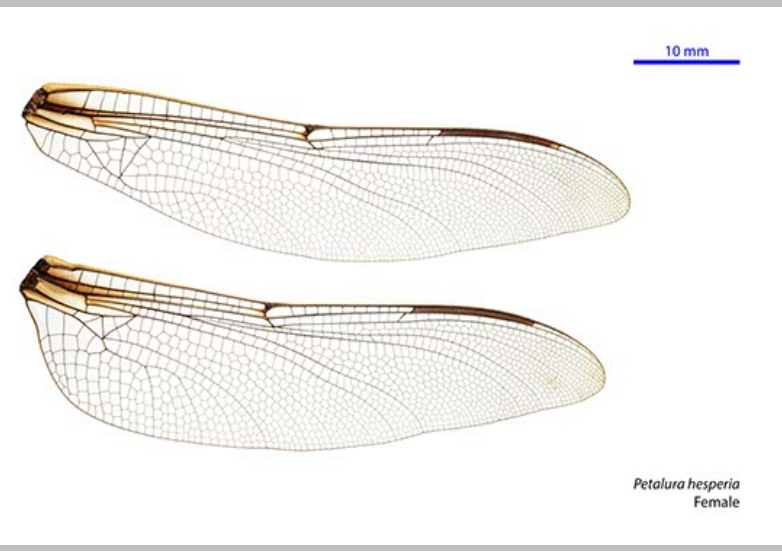

Petalura hesperia female

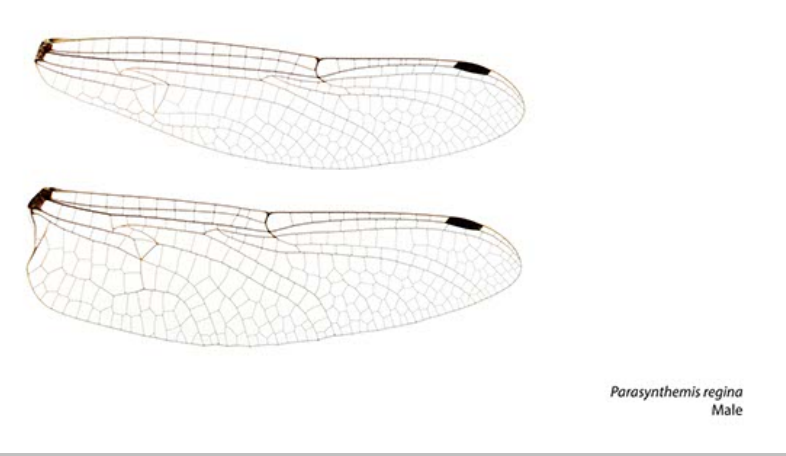

Parasynthemis regina male

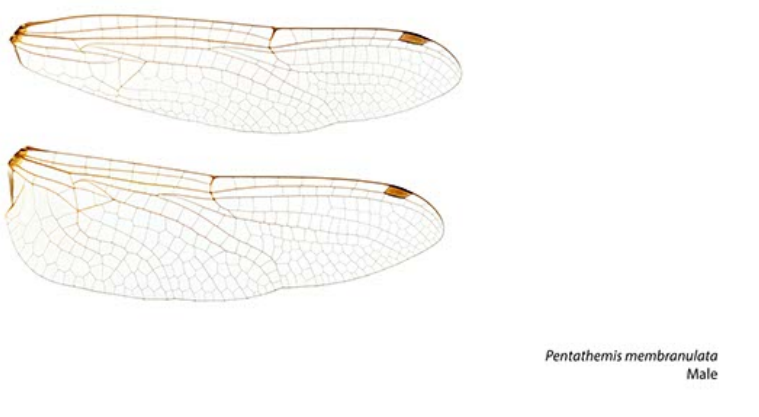

Pentathemis membranulata male

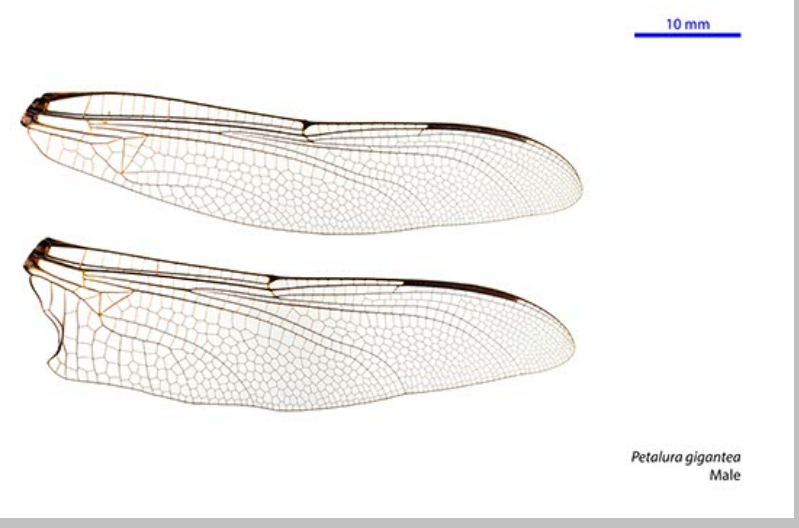

Petalura gigantea male

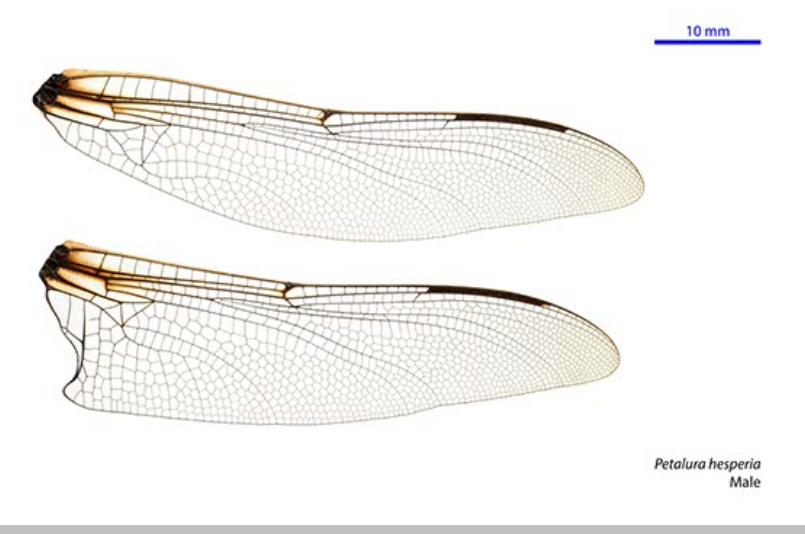

Petalura hesperia male 


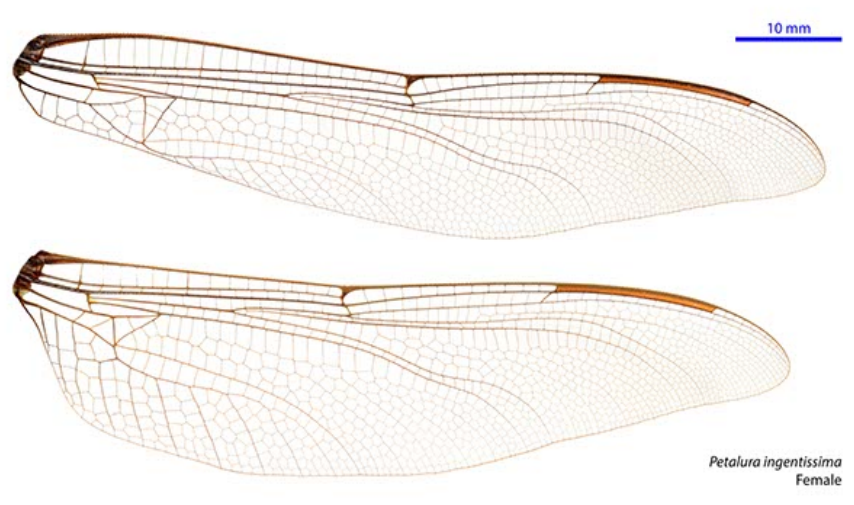

Petalura ingentissima female

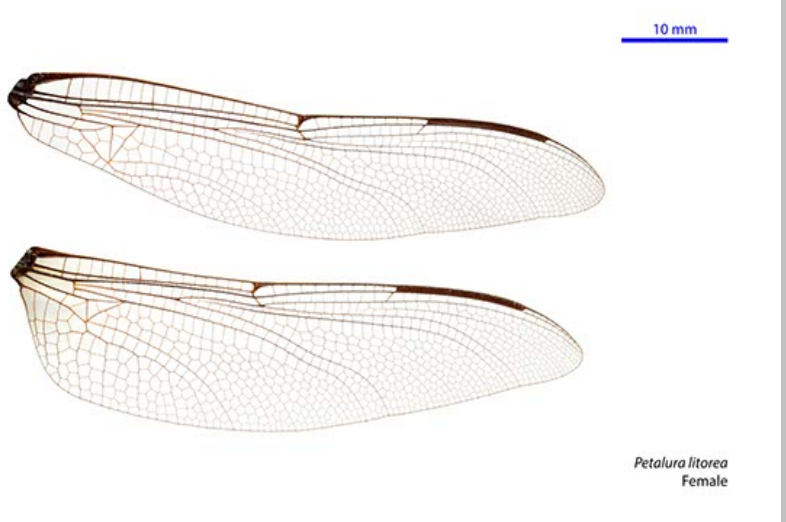

Petalura litorea female

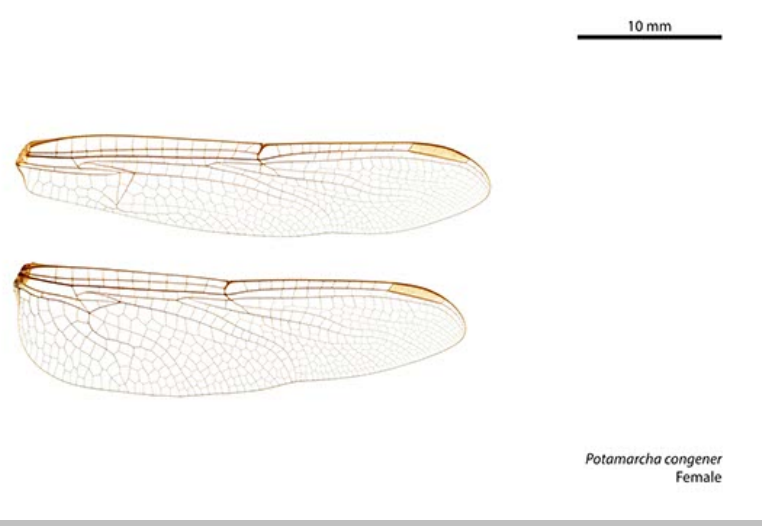

Potamarcha congener female
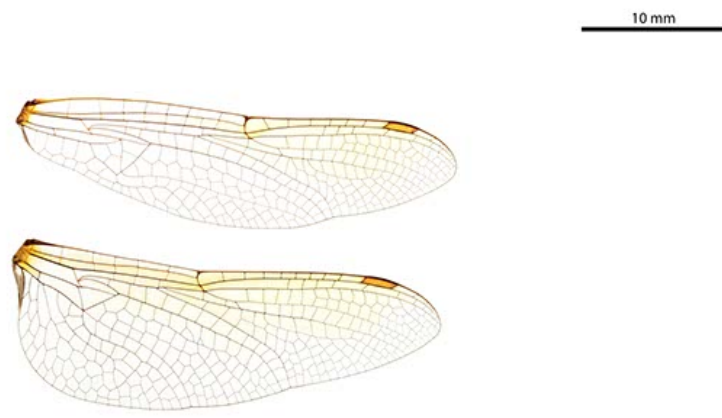

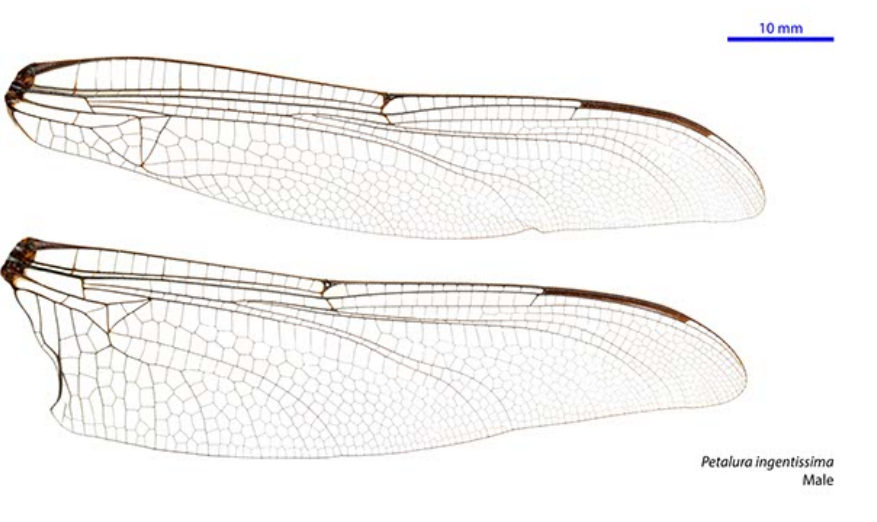

Petalura ingentissima male

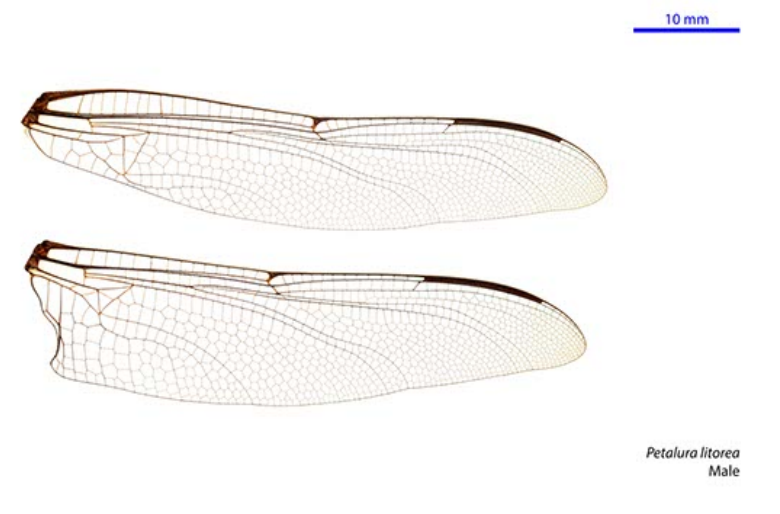

Petalura litorea male

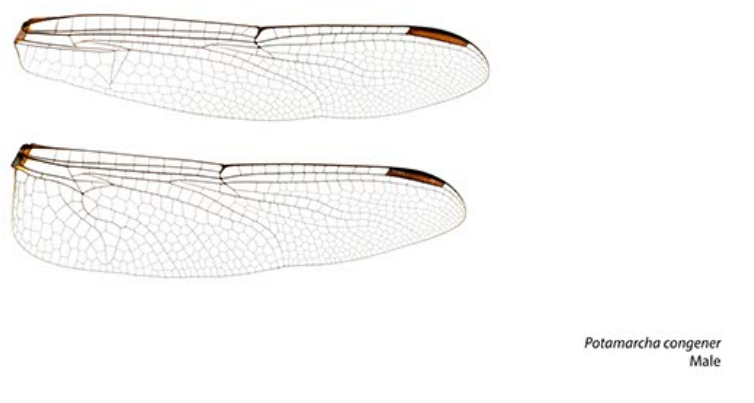

Potamarcha congener male

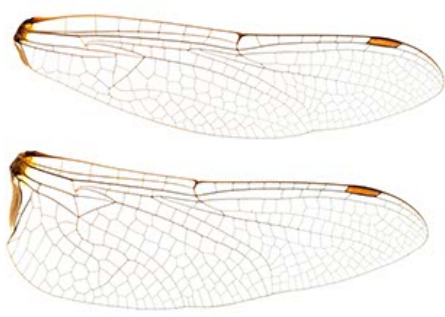



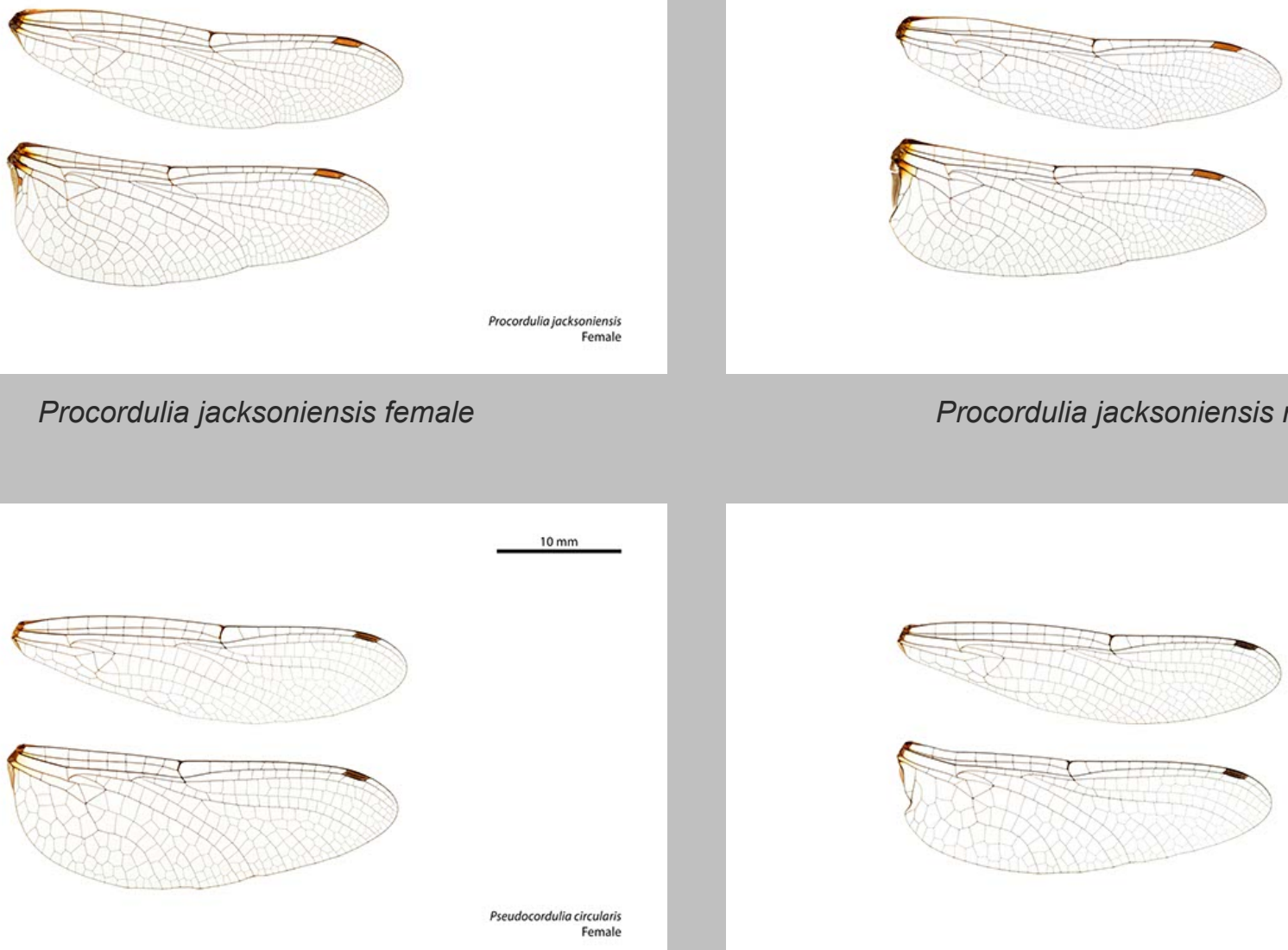

Pseudocordulia circularis female

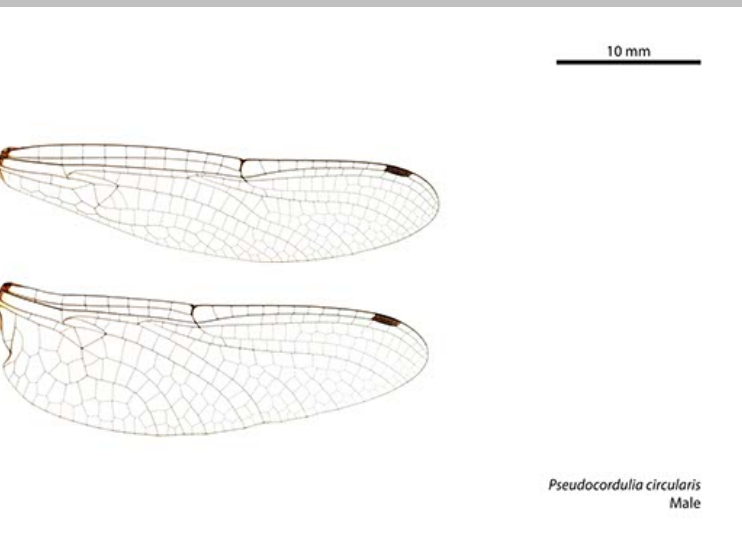

\section{Pseudocordulia circularis male}

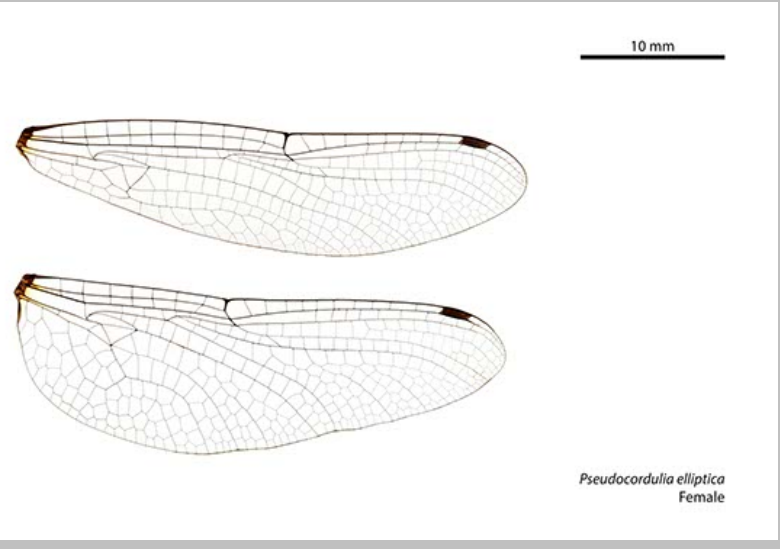

Pseudocordulia elliptica female

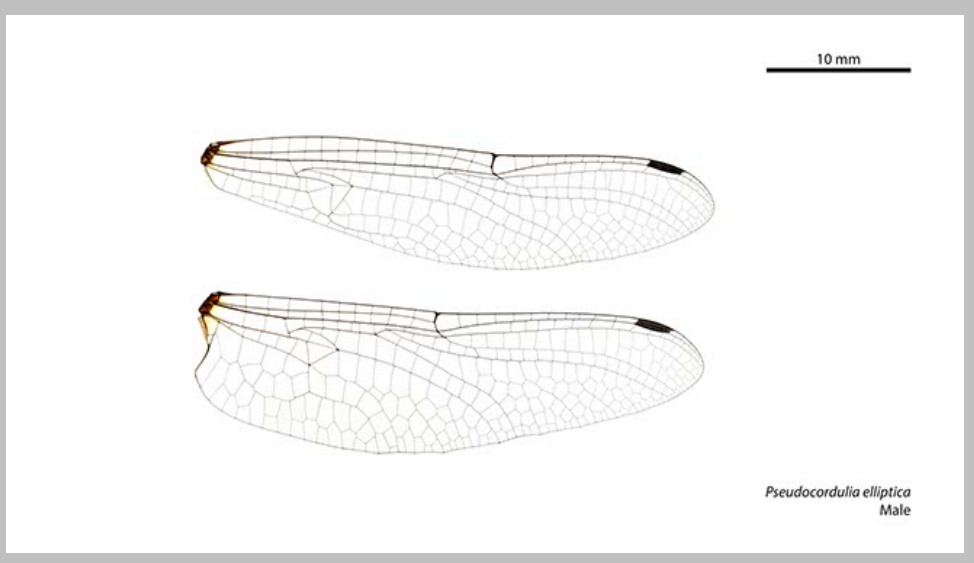

Pseudocordulia elliptica male

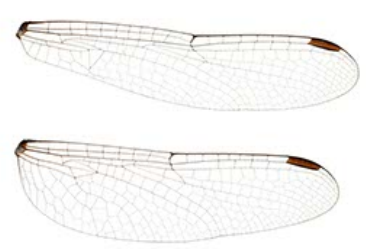




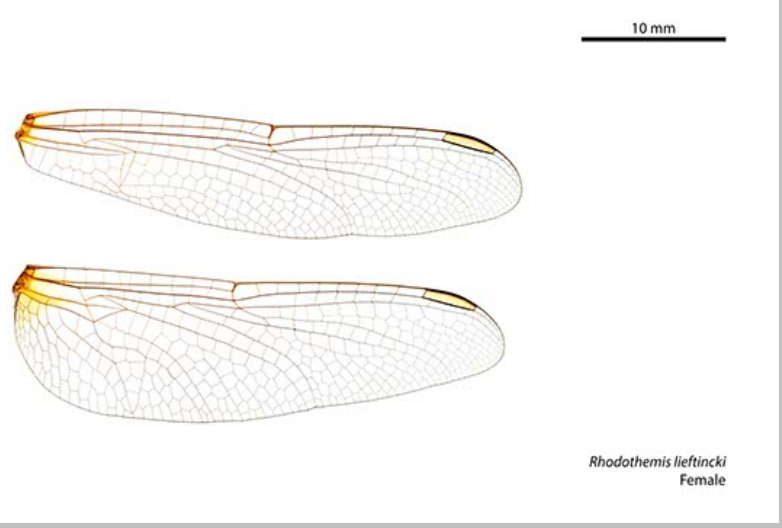

Rhodothemis lieftincki female
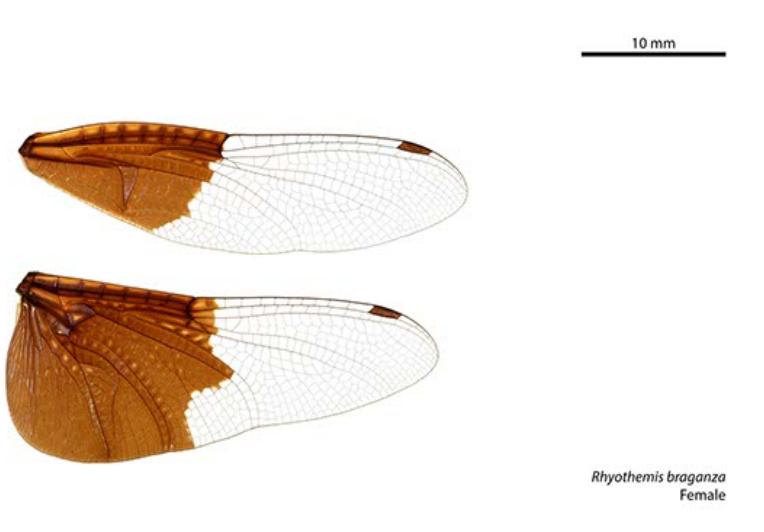

Rhyothemis braganza female

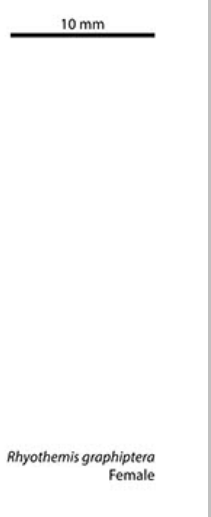

Rhyothemis graphiptera female
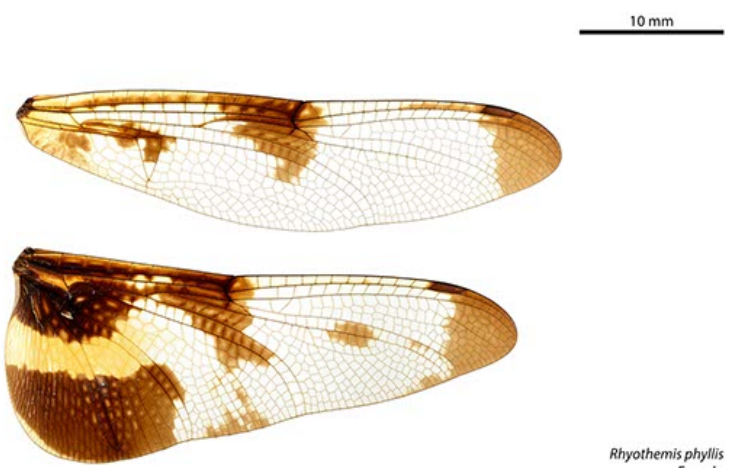

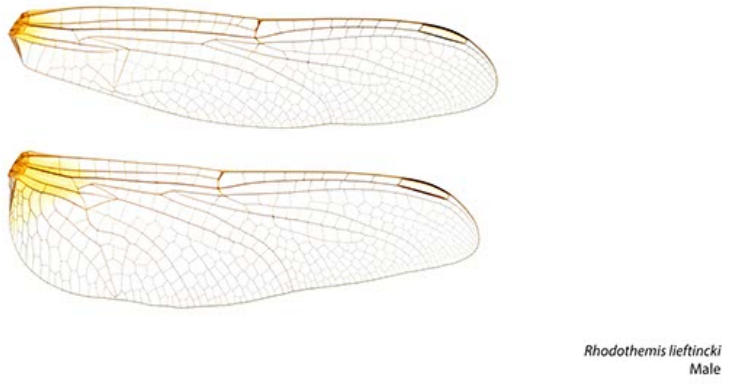

Rhodothemis lieftincki male

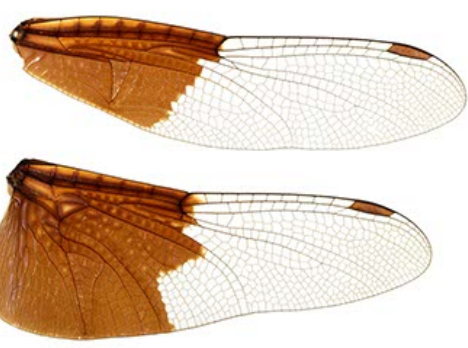

Rhyothemis braganza
Male

Rhyothemis braganza male
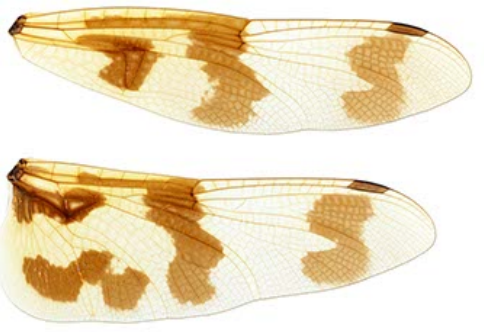

Rhyothemis graphiptera
Male

Rhyothemis graphiptera male

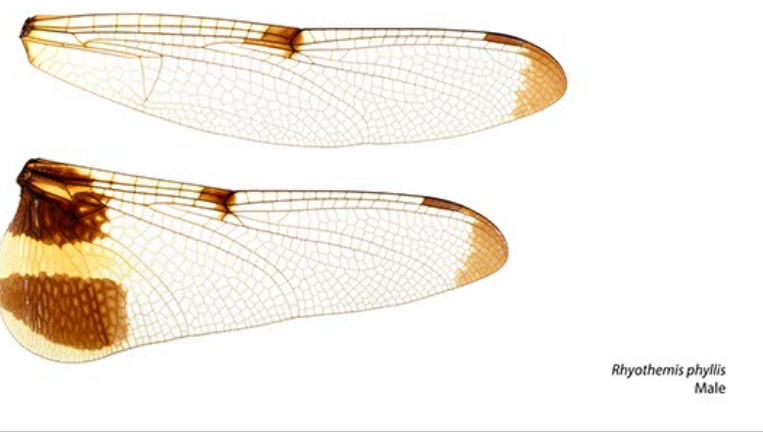

Rhyothemis phyllis male 

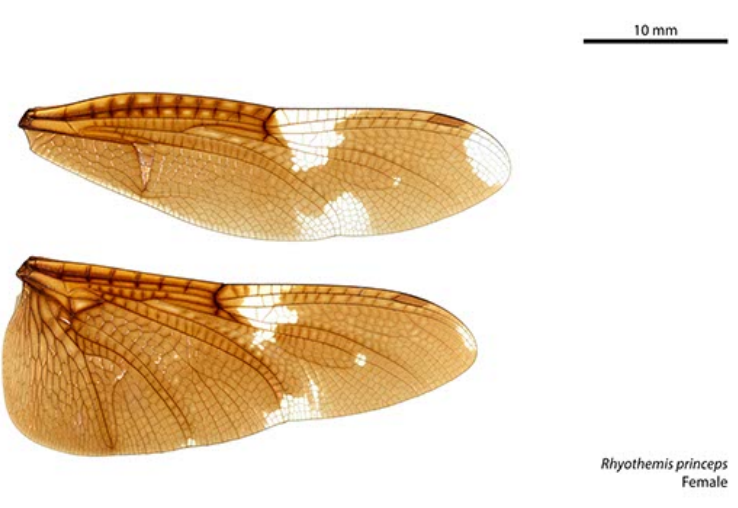

Rhyothemis princeps female

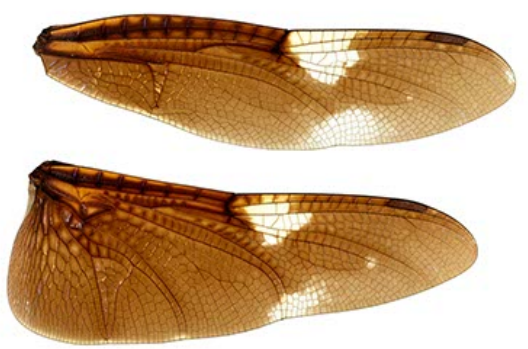

Rhyothemis princeps male

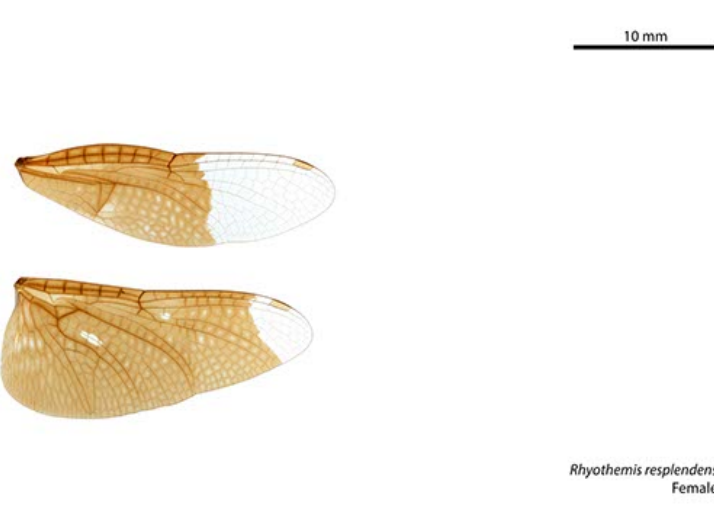

Rhyothemis resplendens female

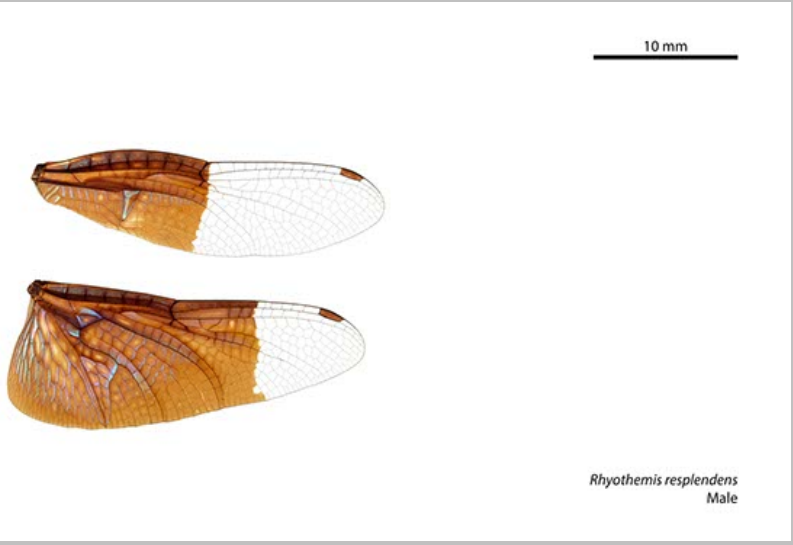

Rhyothemis resplendens male
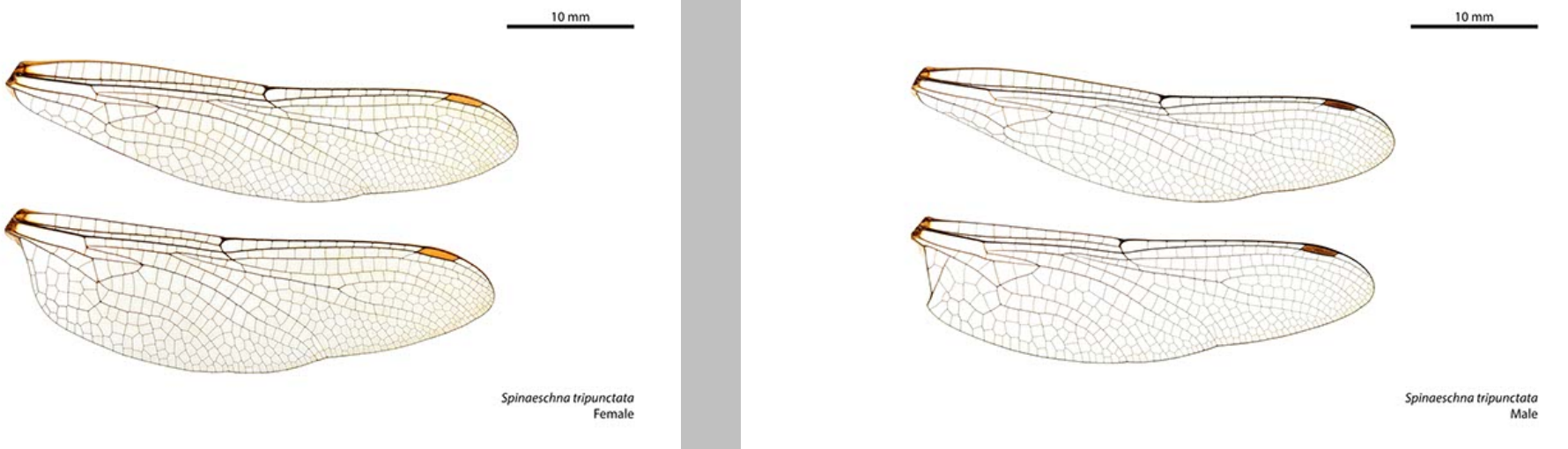

Spinaeschna tripunctata female

\section{Spinaeschna tripunctata male}
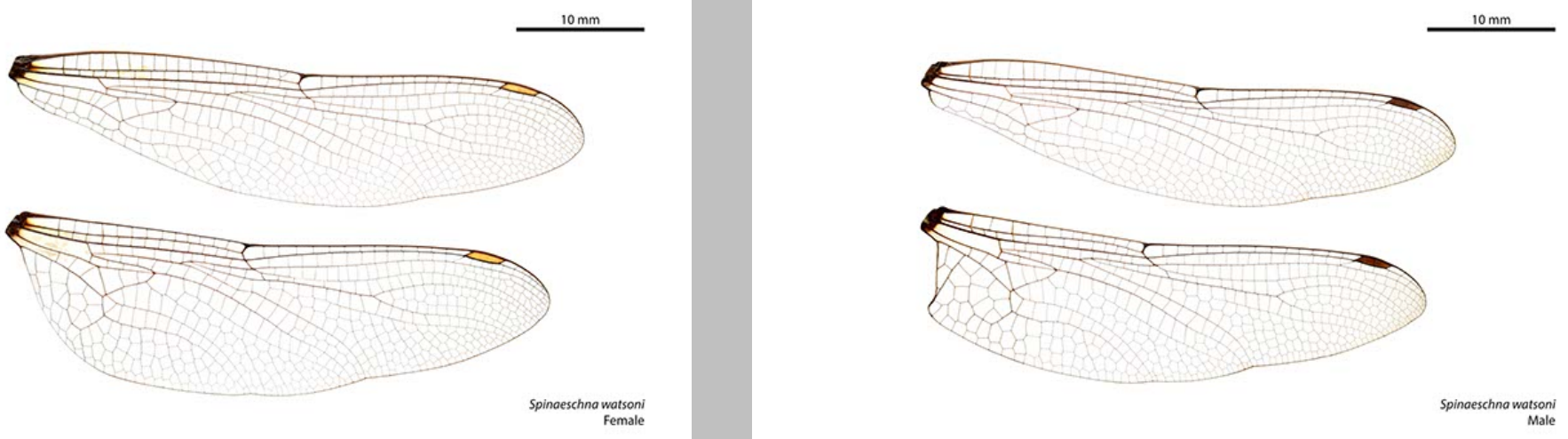

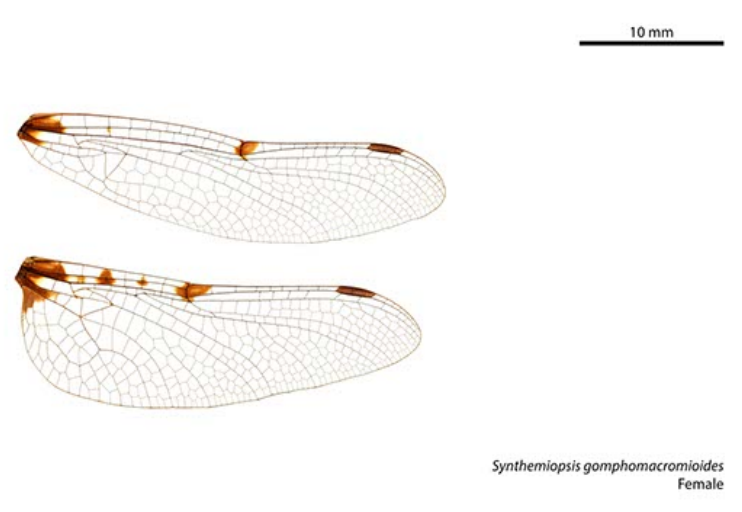

Synthemiopsis gomphomacromioides female

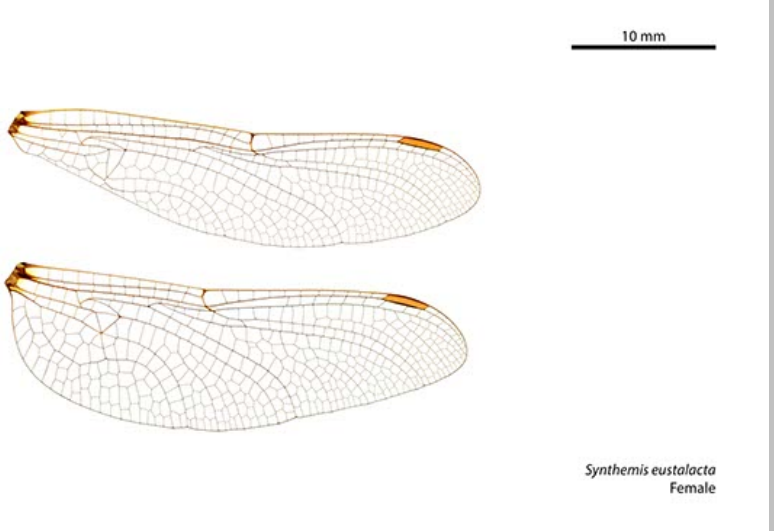

Synthemis eustalacta female

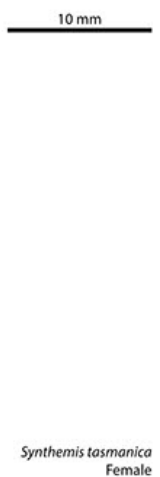

Synthemis tasmanica female

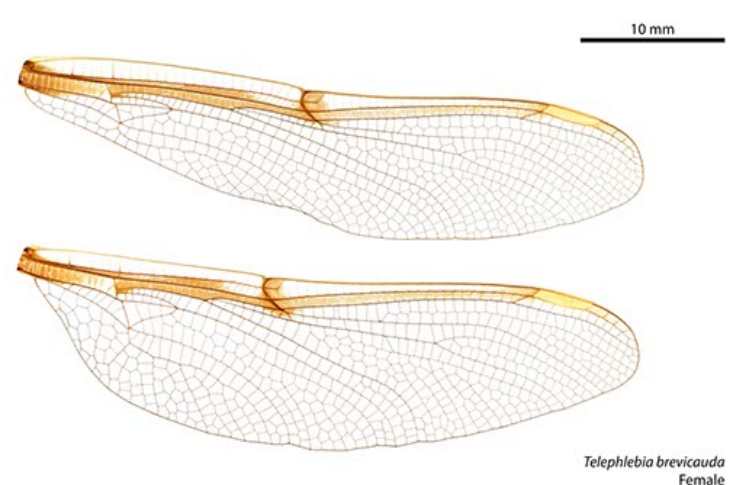

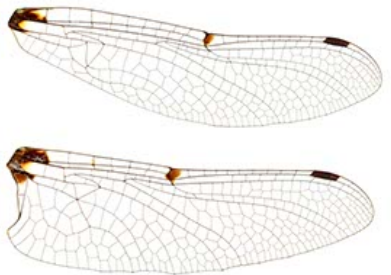

Synthemiopsis gomphomacromioides
Male

Synthemiopsis gomphomacromioides male
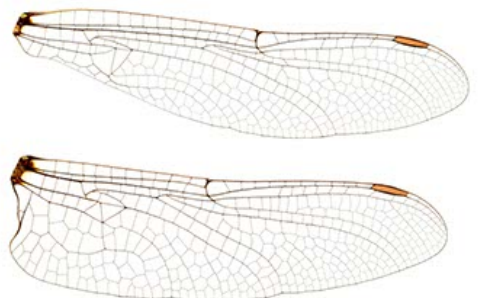

\section{Synthemis eustalacta male}

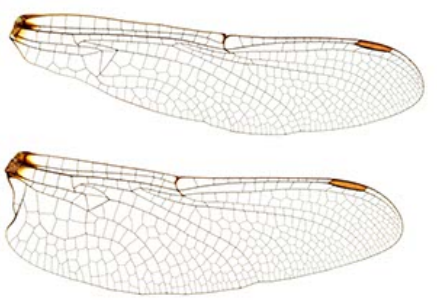

Synthemistasmanic

Synthemis tasmanica male

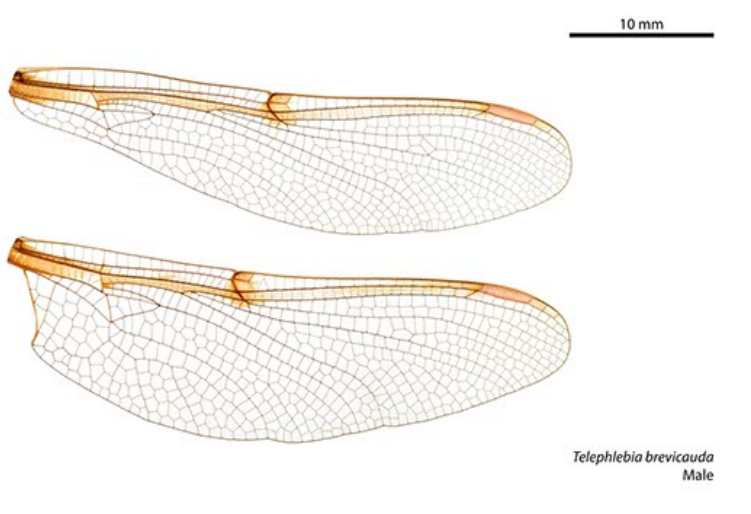

Telephlebia brevicauda male 


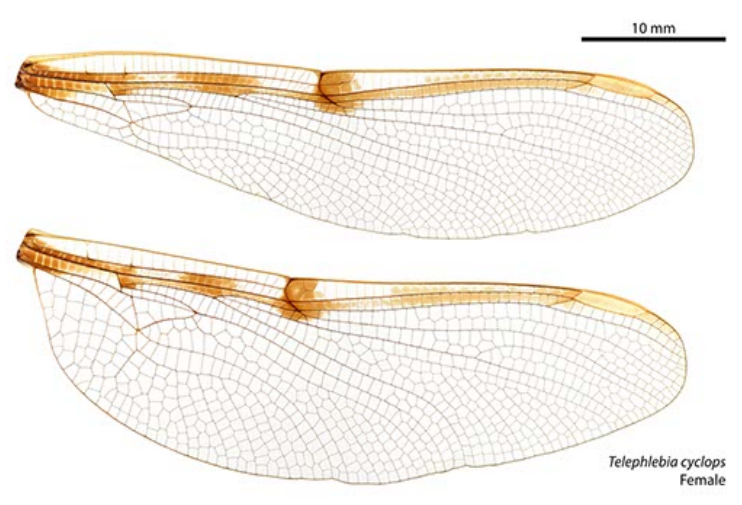

Telephlebia cyclops female

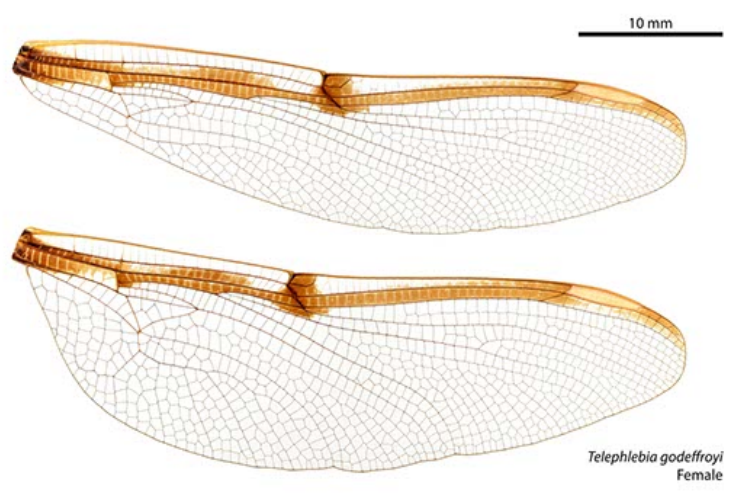

Telephlebia godeffroyi female

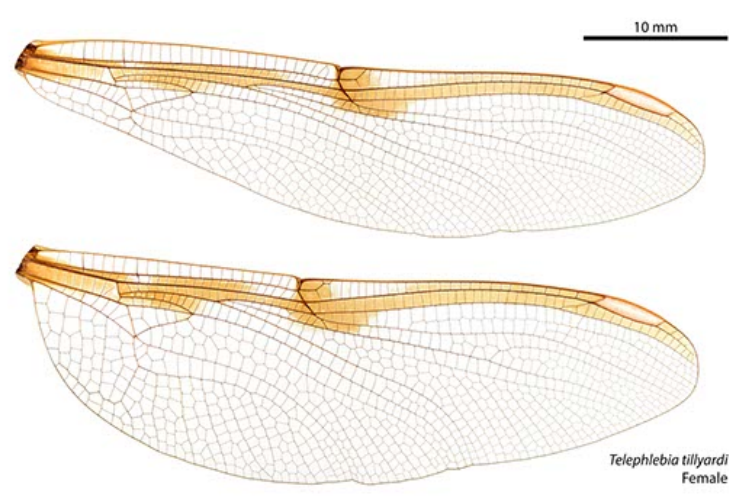

Telephlebia tillyardi female

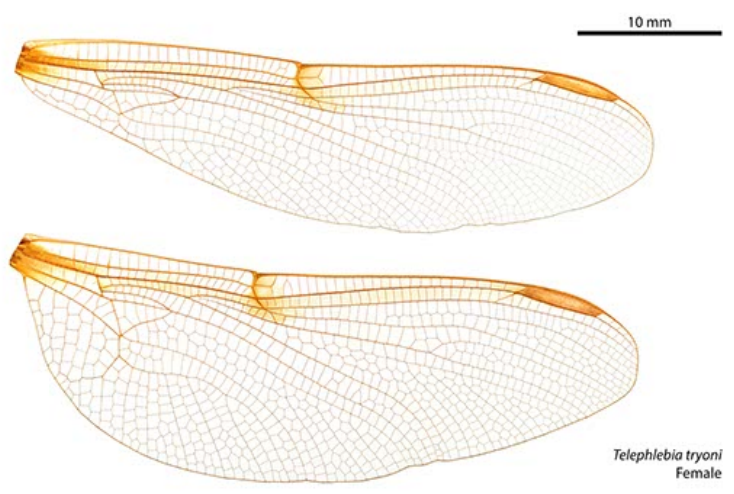

Telephlebia tryoni female

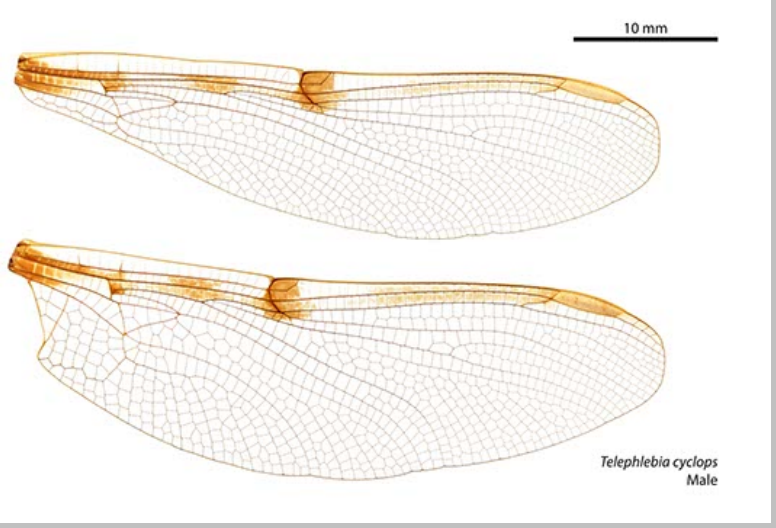

Telephlebia cyclops male

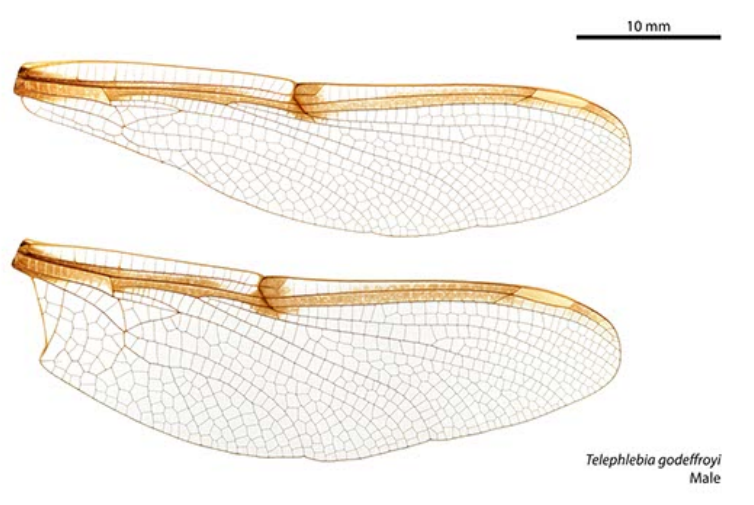

Telephlebia godeffroyi male

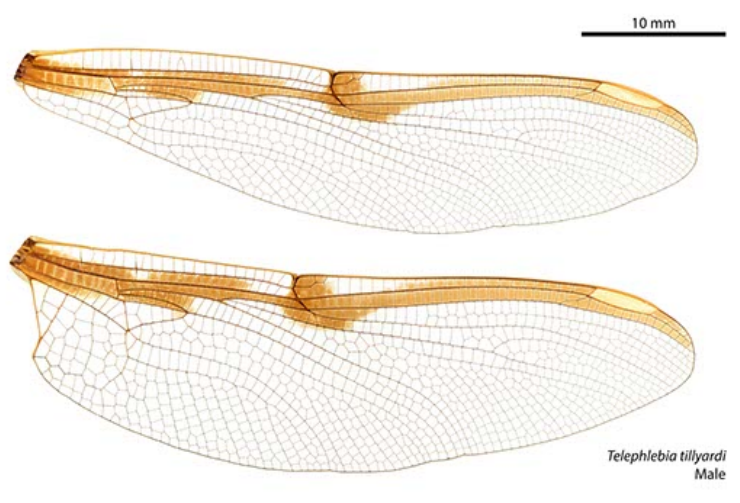

Telephlebia tillyardi male

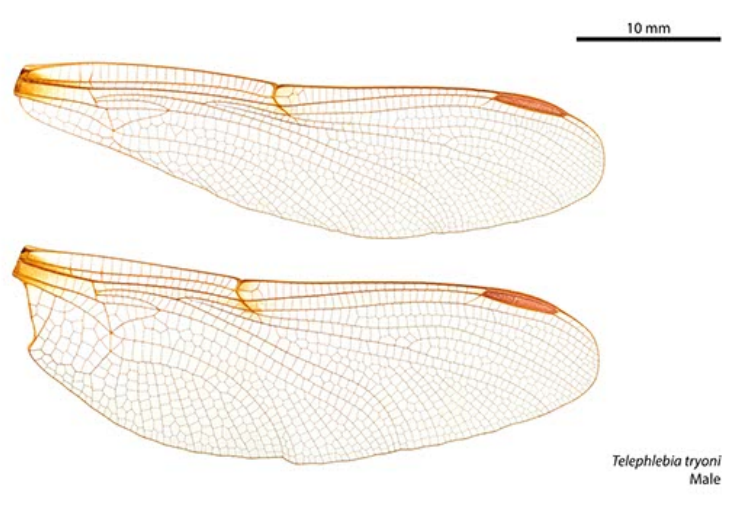

Telephlebia tryoni male 


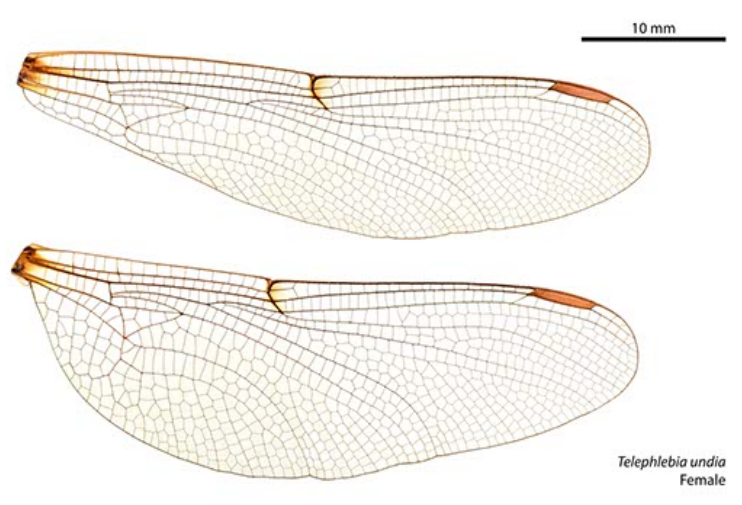

Telephlebia undia female

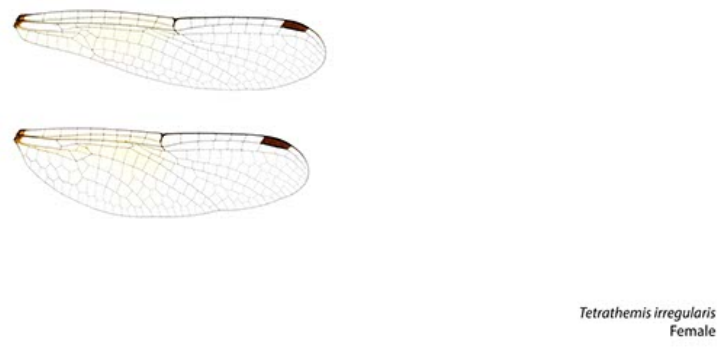

Tetrathemis irregularis female

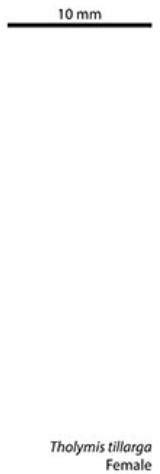

Tholymis tillarga female

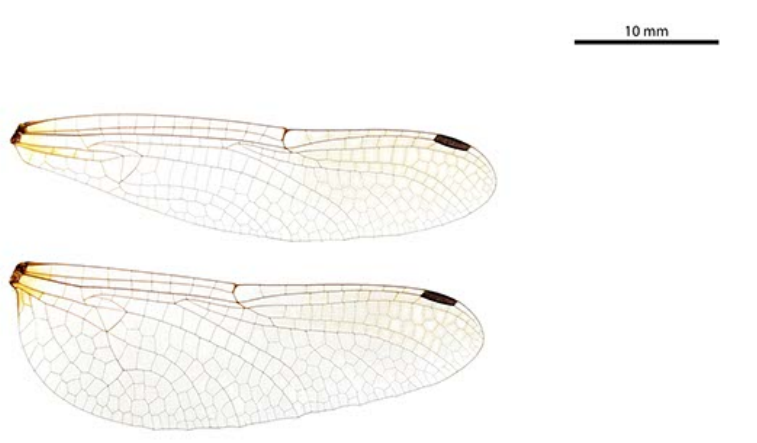

Tonyosynthemis claviculata
Telephlebia undia male

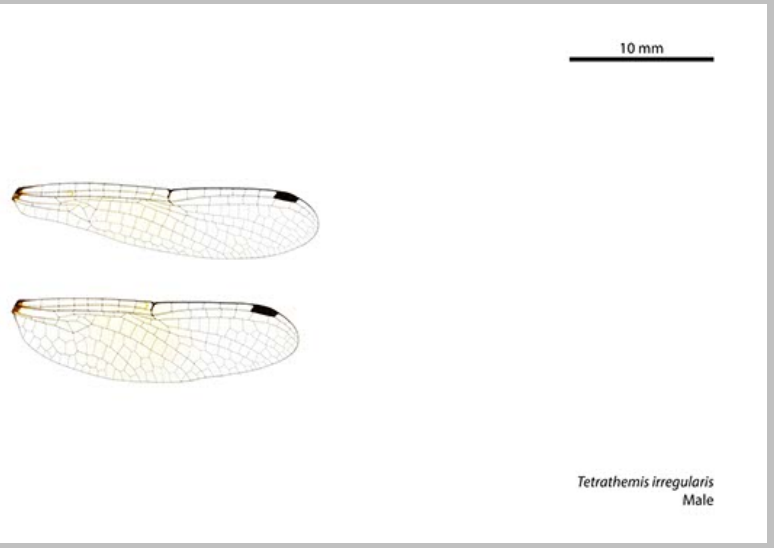

Tetrathemis irregularis male

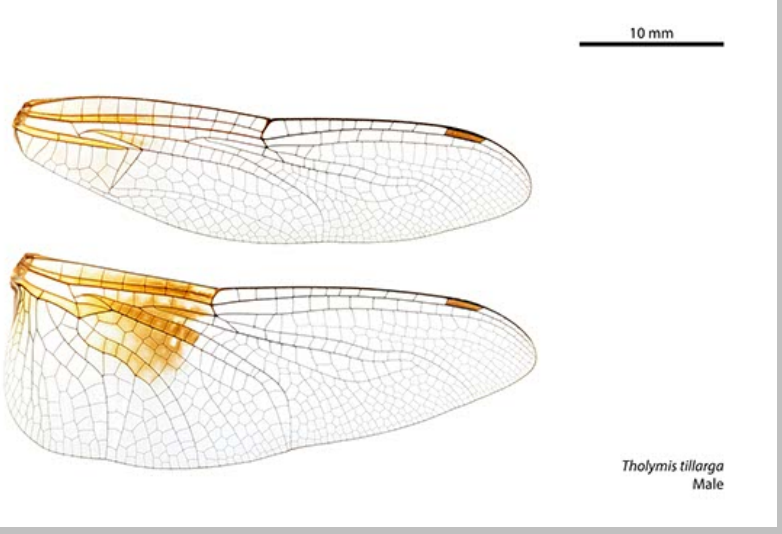

Tholymis tillarga male

Tonyosynthemis claviculata 


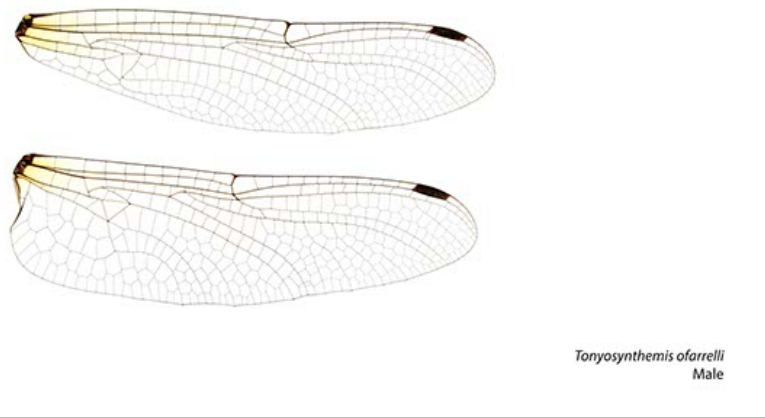

Tonyosynthemis ofarrelli male
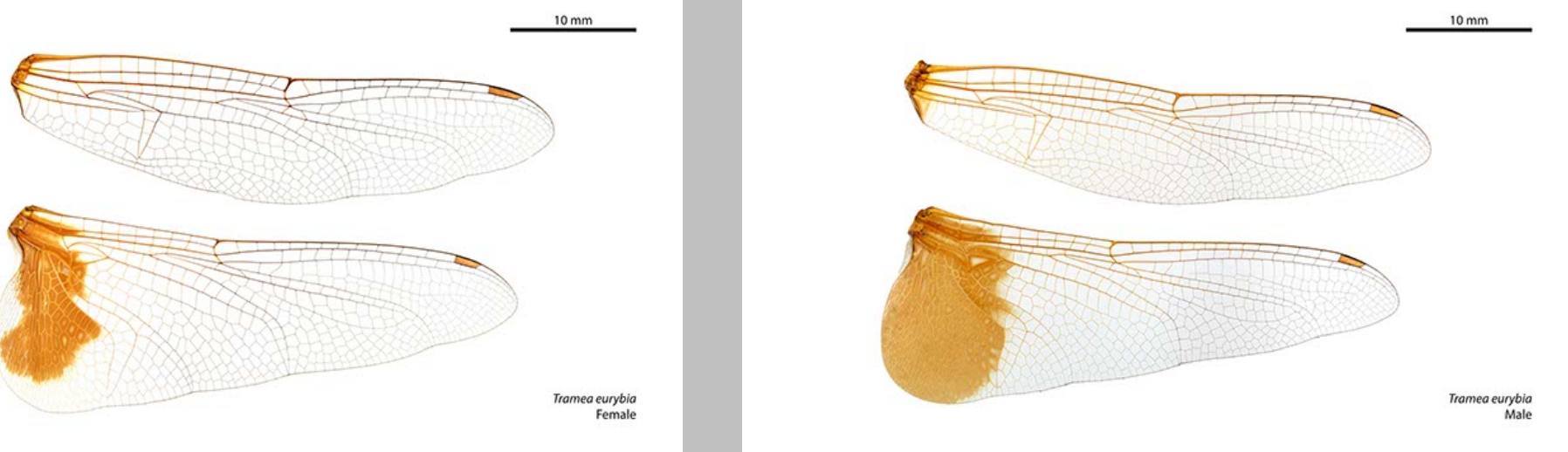

Tramea eurybia female

Tramea eurybia male
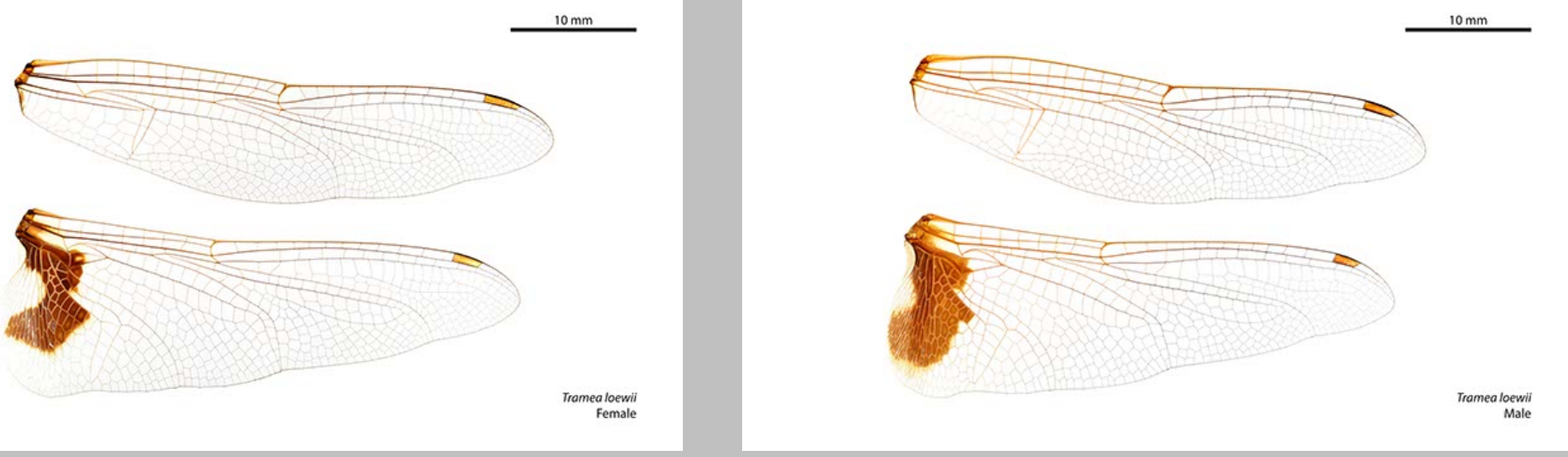

Tramea loewii female

Tramea loewii male
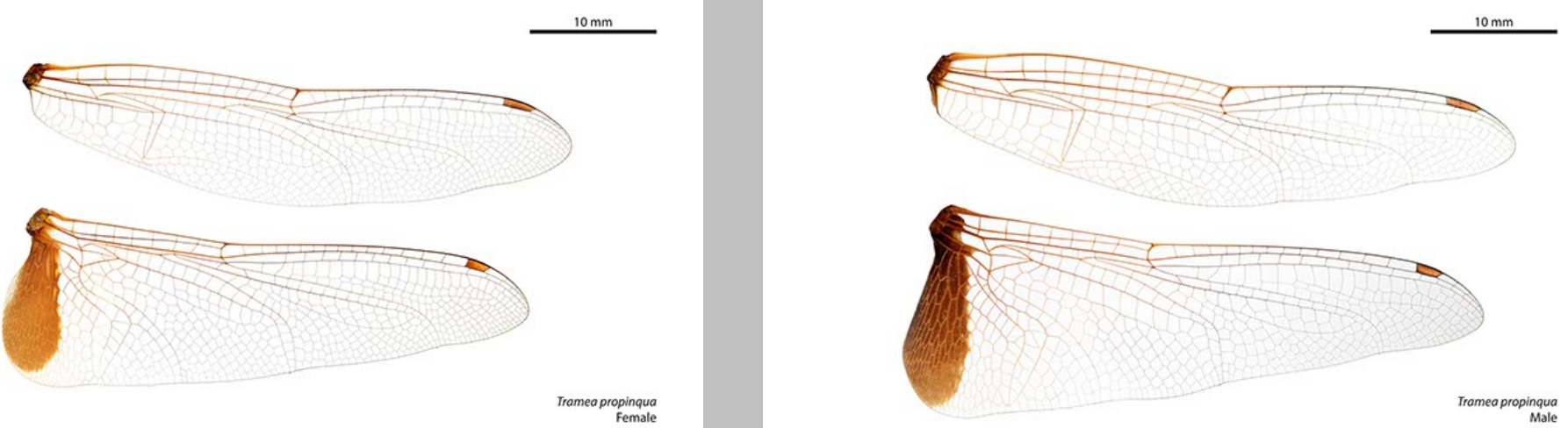


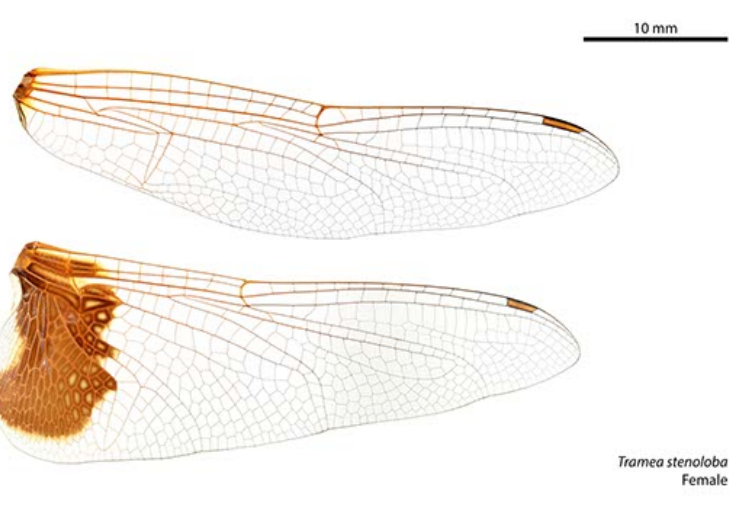

Tramea stenoloba female

Urothemis aliena female

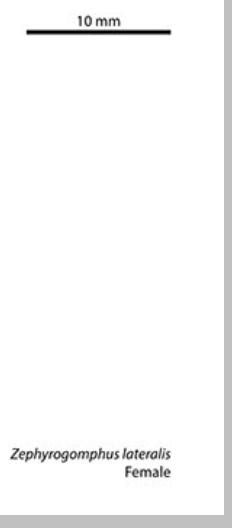

Zephyrogomphus lateralis female

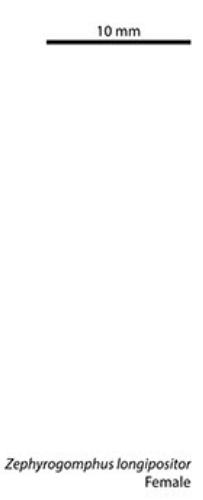

Zephyrogomphus longipositor female

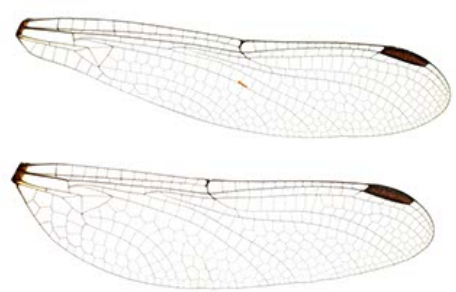

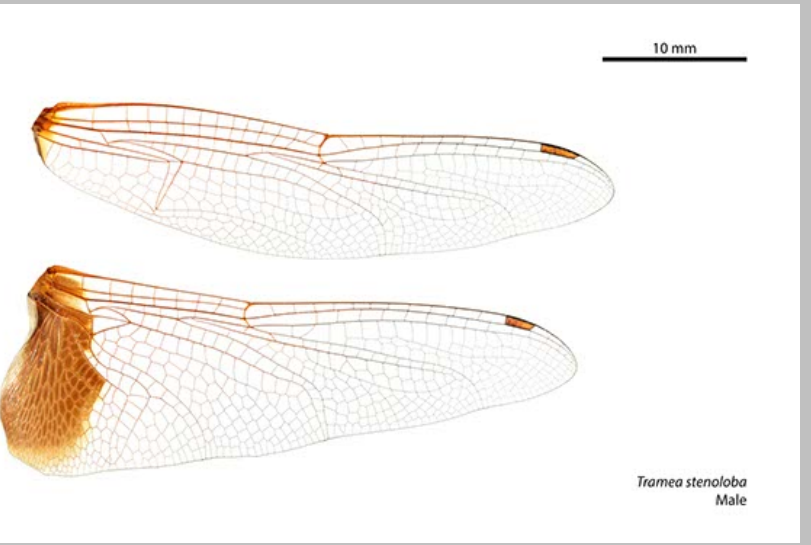

Tramea stenoloba male

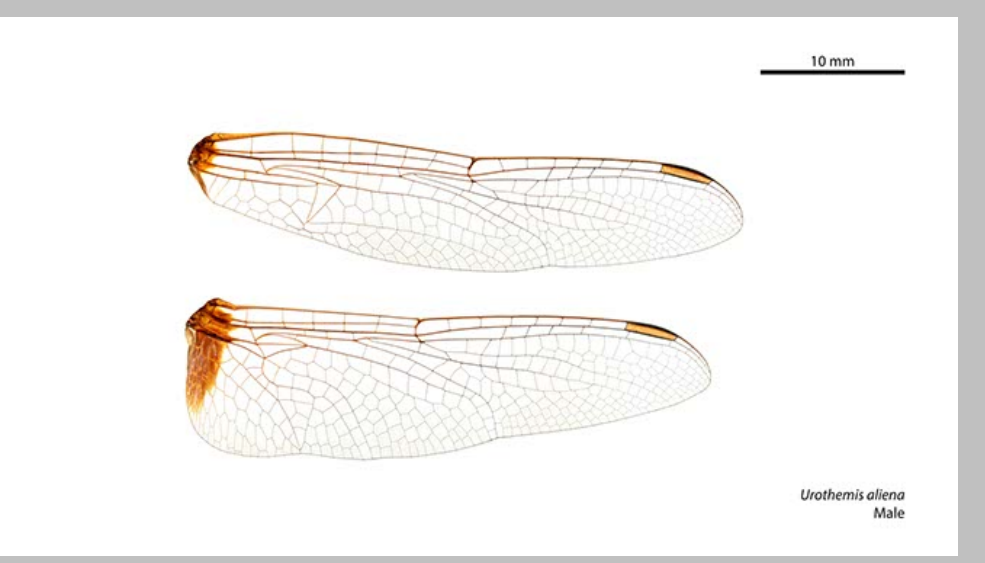

Urothemis aliena male

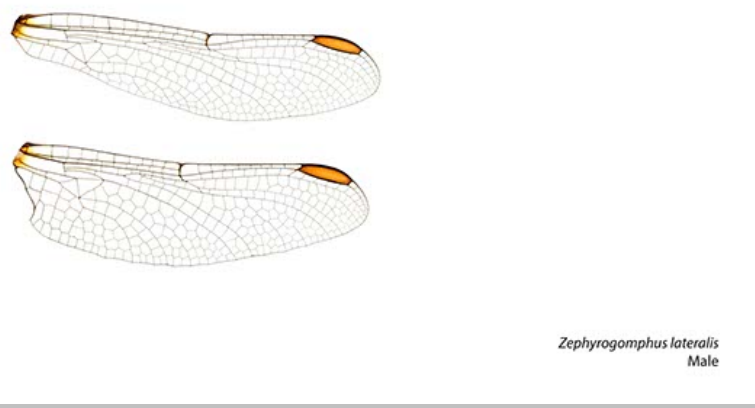

Zephyrogomphus lateralis male 


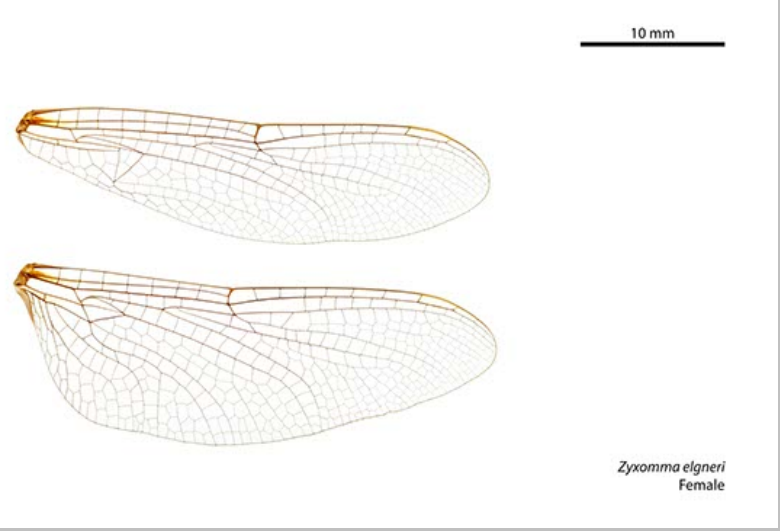

Zyxomma elgneri female

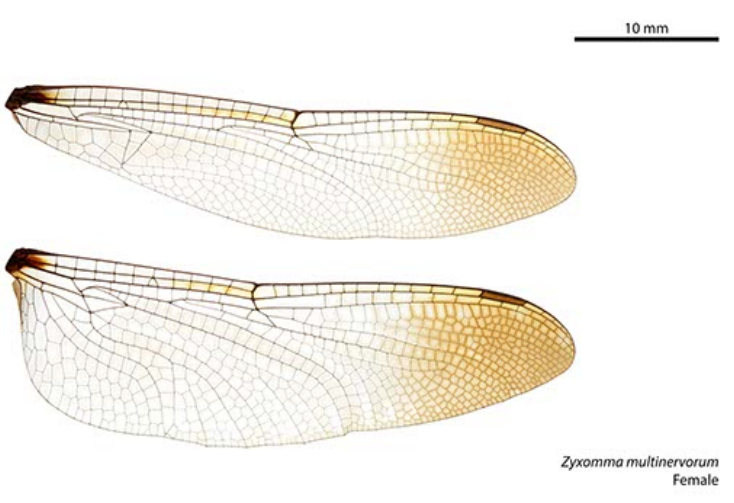

Zyxomma multinervorum female

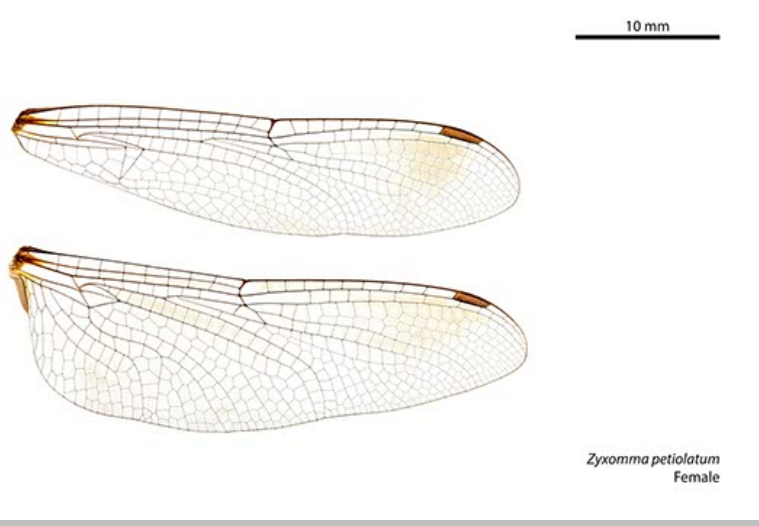

Zyxomma petiolatum female
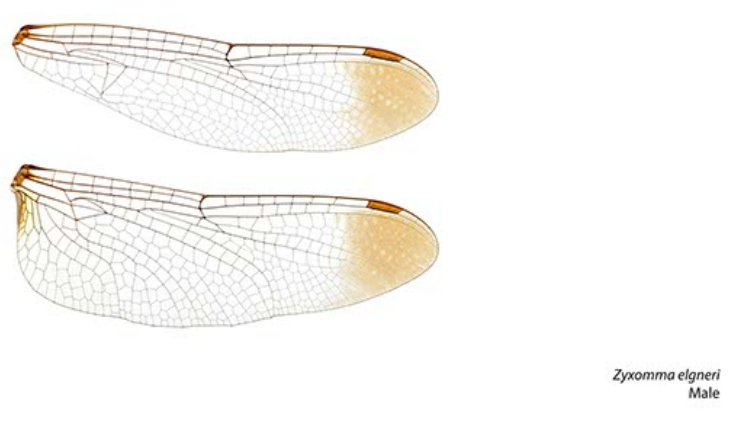

\section{Zyxomma elgneri male}

\section{Zyxomma multinervorum male}

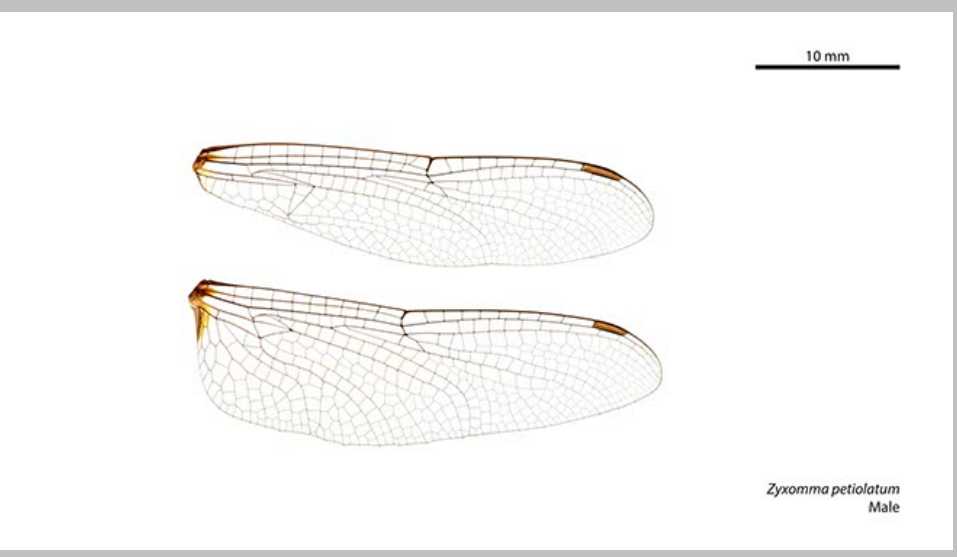

Zyxomma petiolatum male 


\section{Appendix 2-Zygoptera wings}

Photographs of female and male wings of 111 species of Australian damselfly (Zygoptera). Each image shows a pair of right wings, a scale, a taxonomic name and sex. A link is provided in Appendix 3 to a high-resolution photograph of each species.

Images presented below are low resolution "thumbnails" providing a visual ready-reckoner and quick guide to more detailed imagery. The entire photographic library, at highest resolution, is openly accessible to view or download from figshare as either individual images or as a complete set of Australian Odonata wing images (Tann, 2020a-d).

All damselfly images are presented here at a consistent fixed scale for ready comparison between species. 


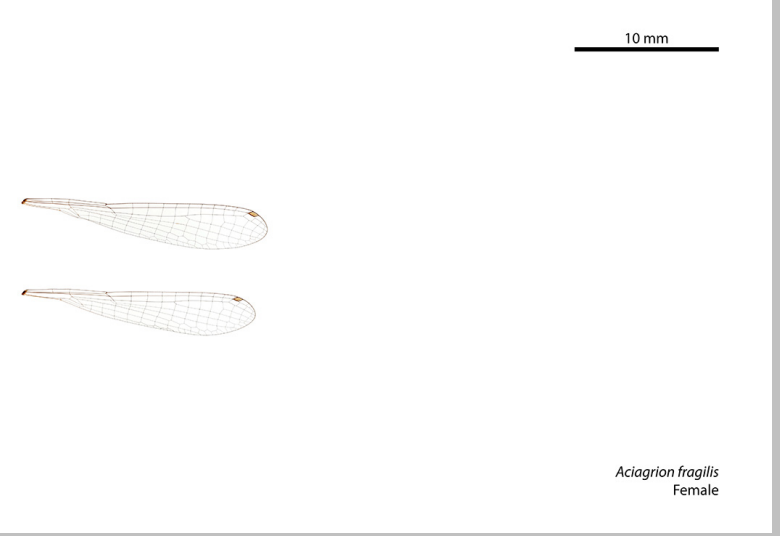

Aciagrion fragilis female

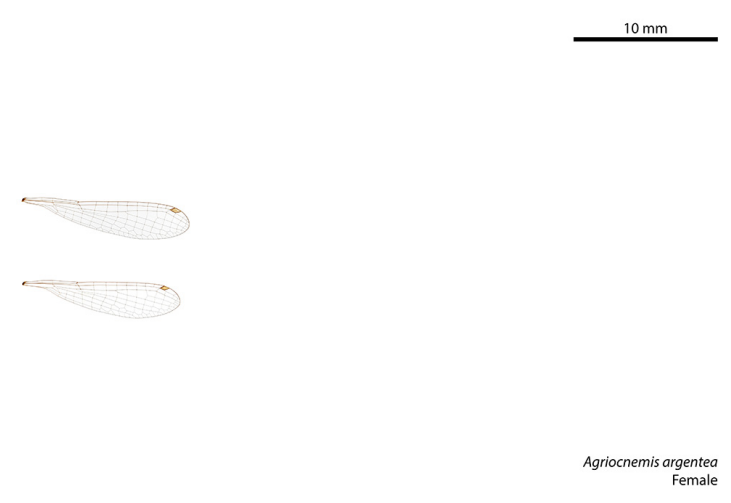

Agriocnemis argentea female

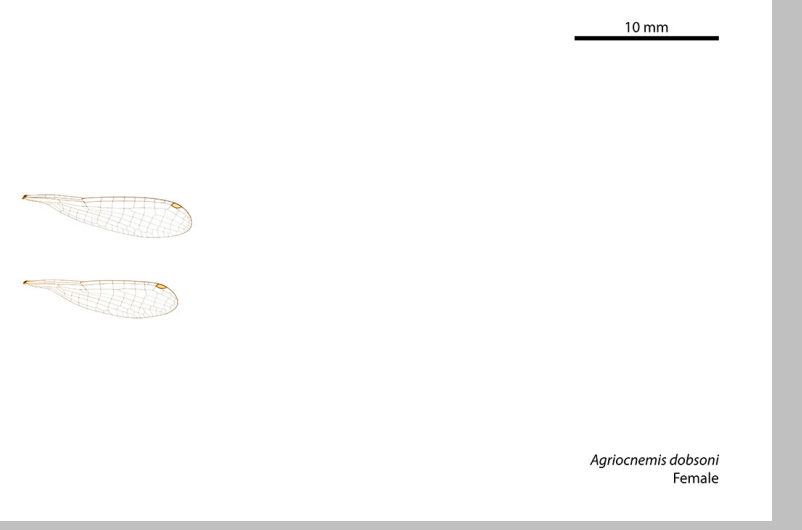

Agriocnemis dobsoni
Female

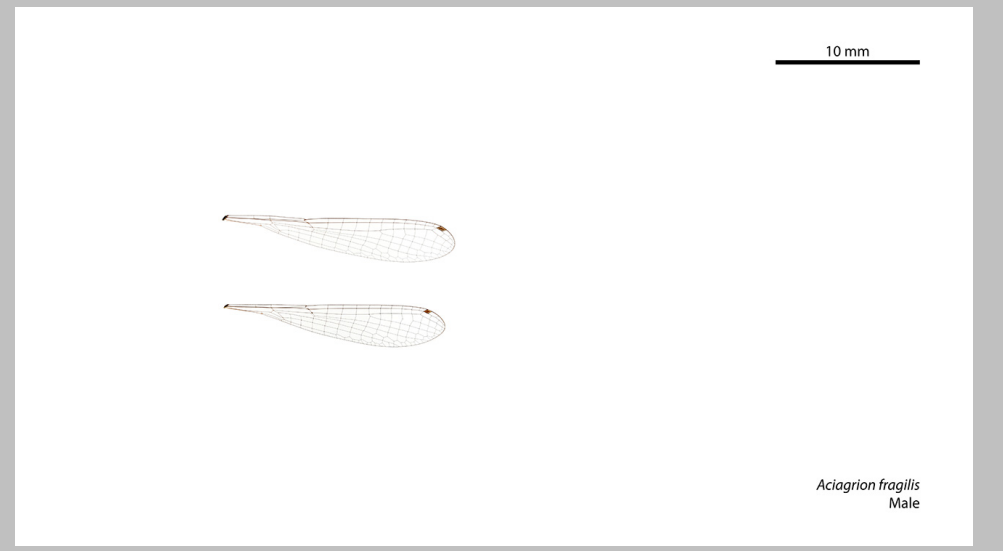

Aciagrion fragilis male
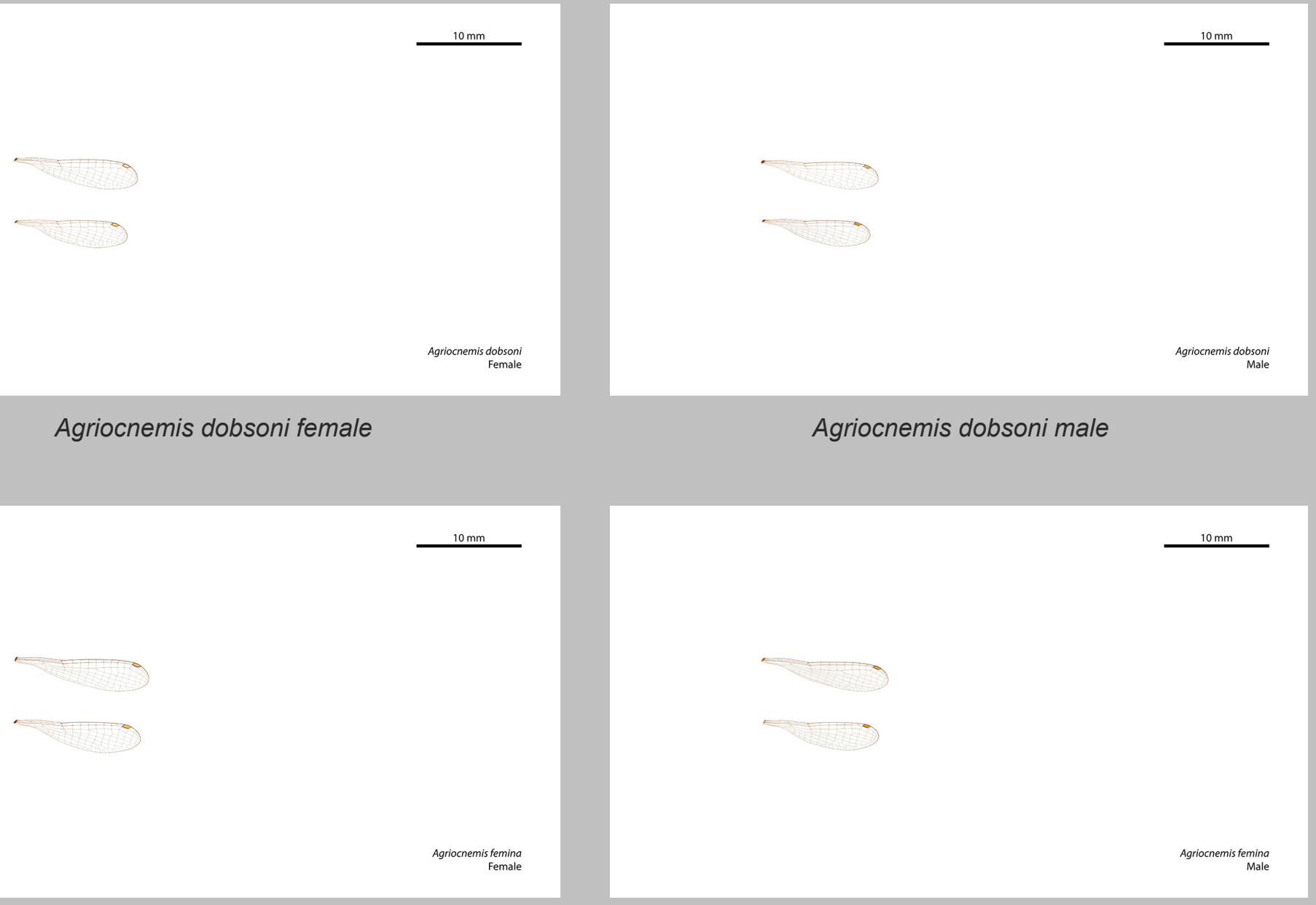


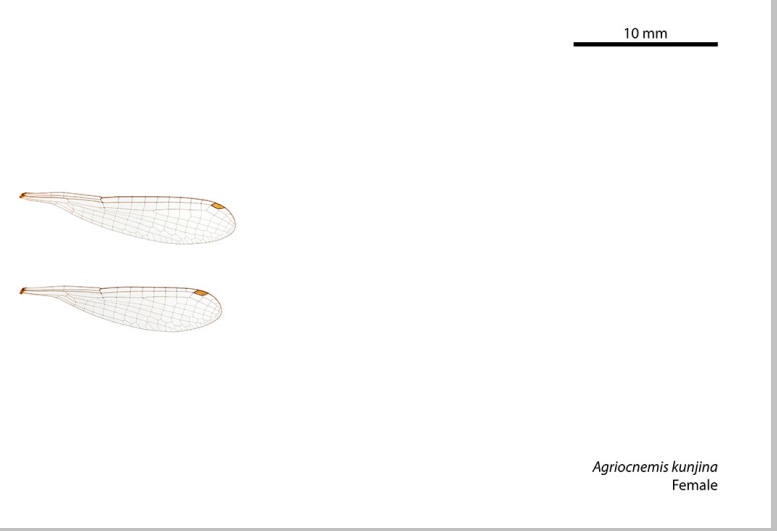

Agriocnemis kunjina female

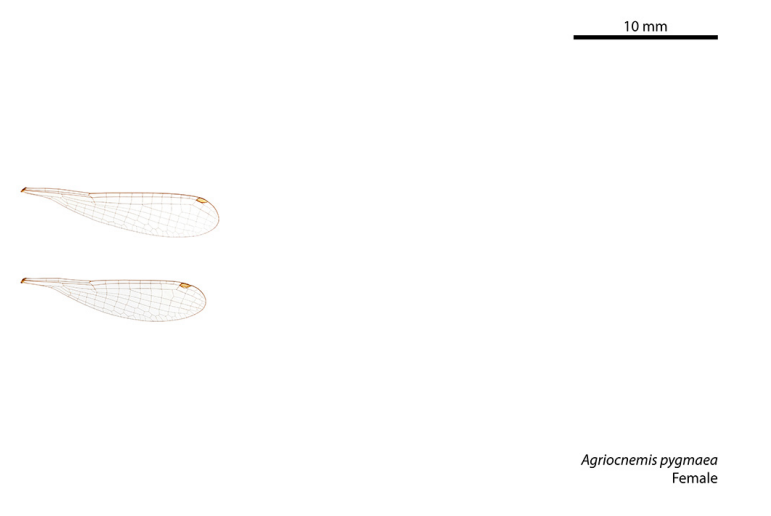

Agriocnemis pygmaea female

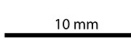

Agriocnemis rubricauda
Female

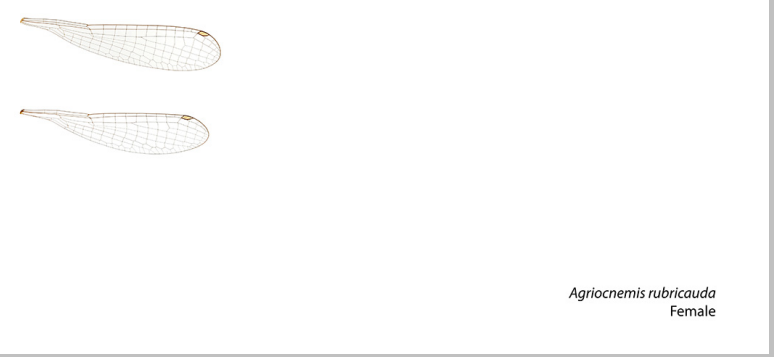

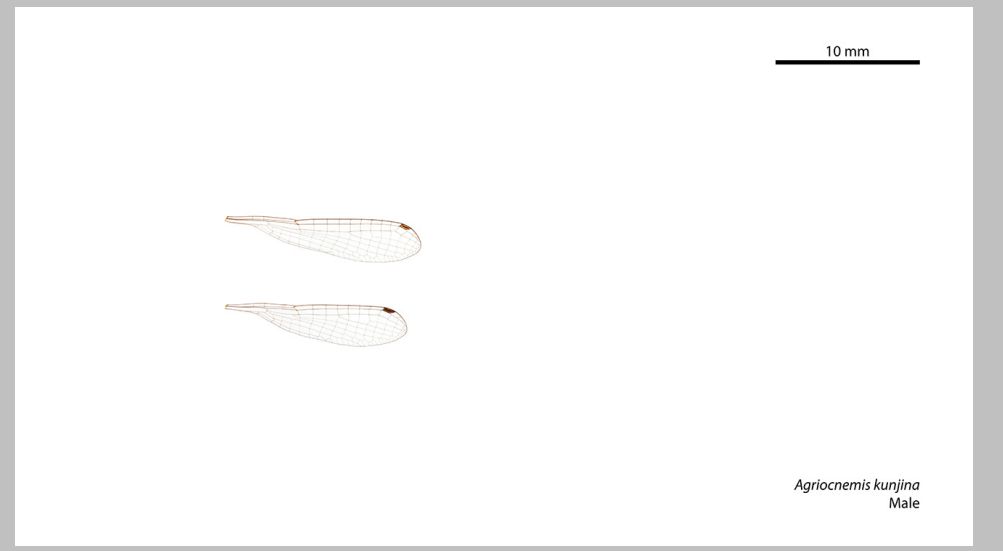

Agriocnemis kunjina male

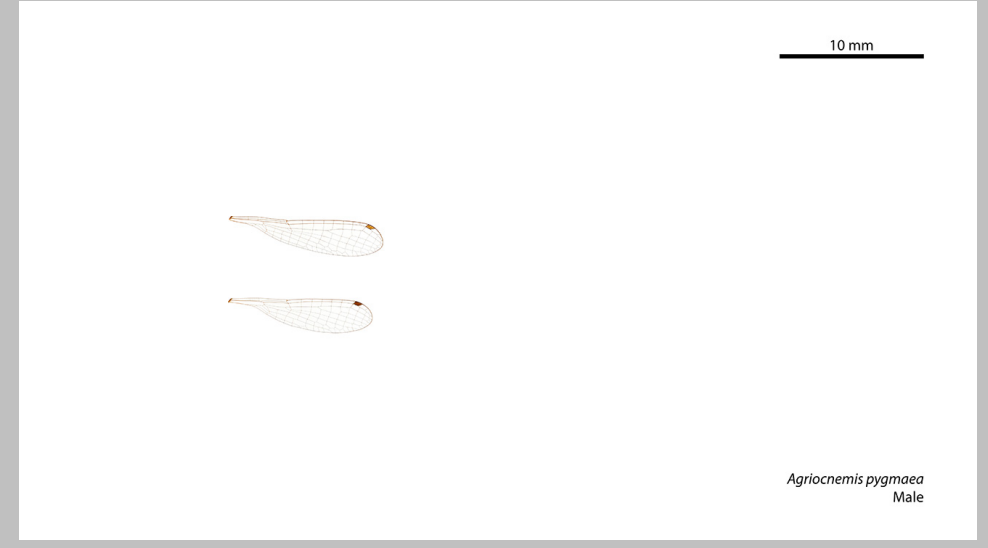

Agriocnemis pygmaea male

Agriocnemis rubricauda female

Agriocnemis rubricauda male
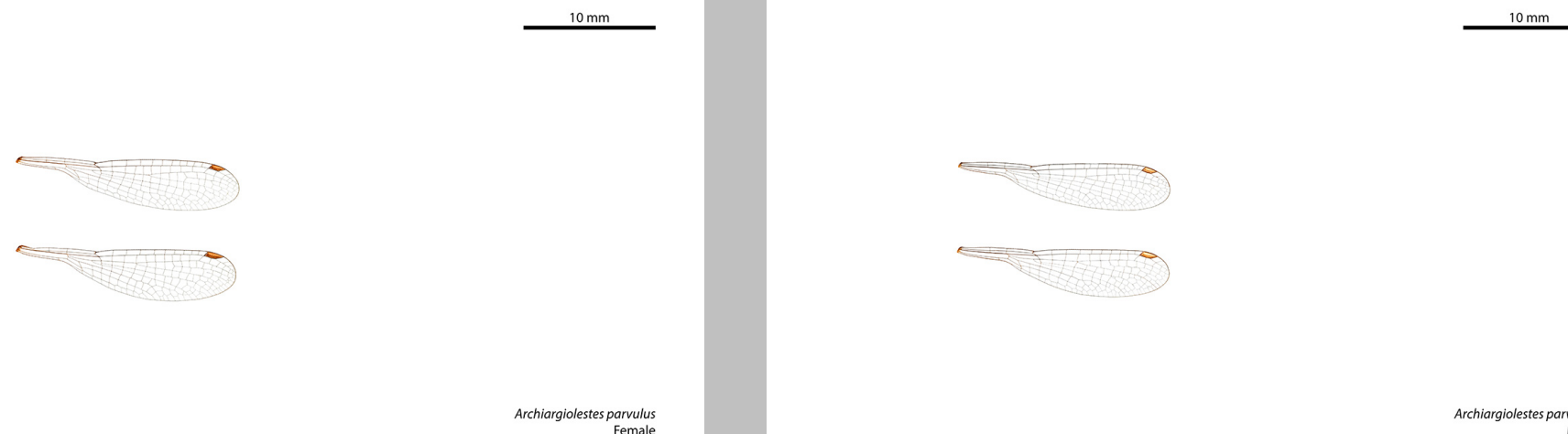


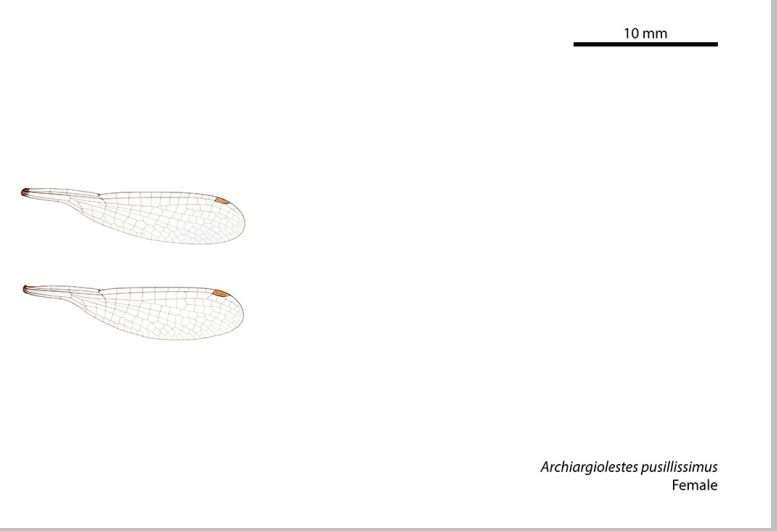

Archiargiolestes pusillissimus female

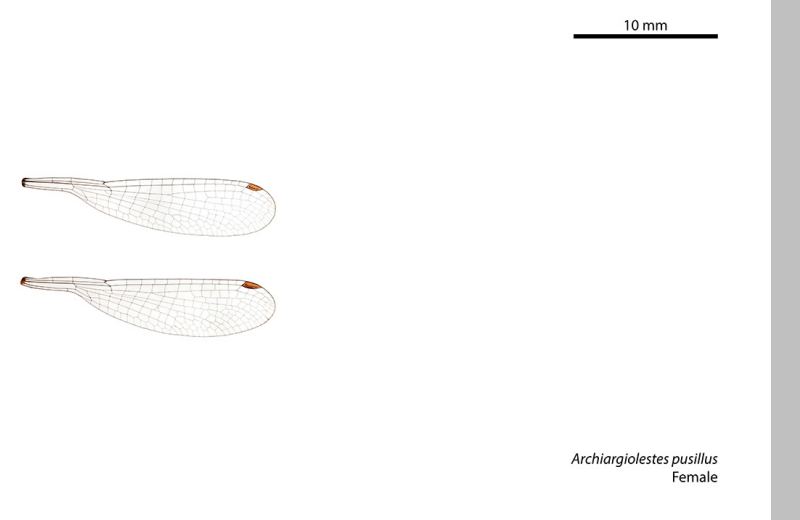

Archiargiolestes pusillus female

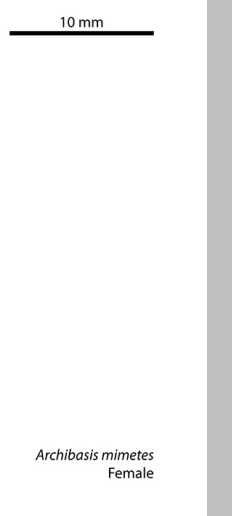

Archibasis mimetes female

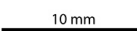




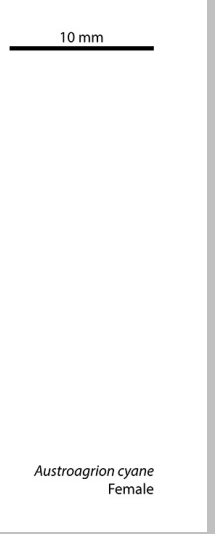

Austroagrion cyane female

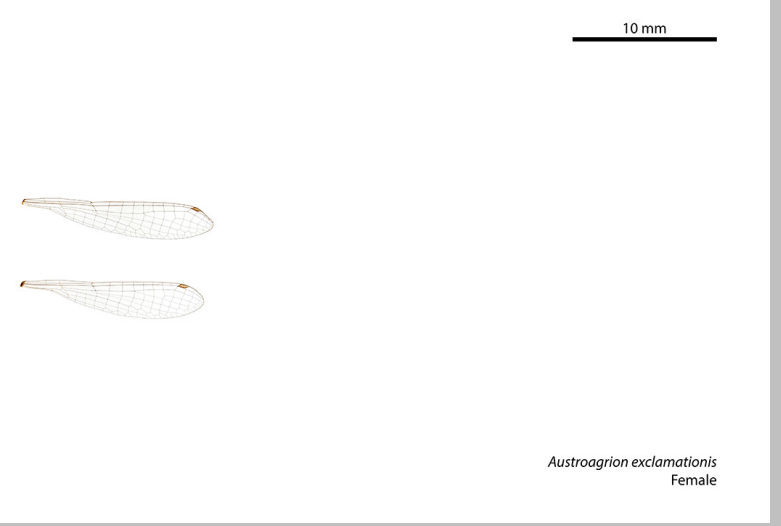

Austroagrion exclamationis female

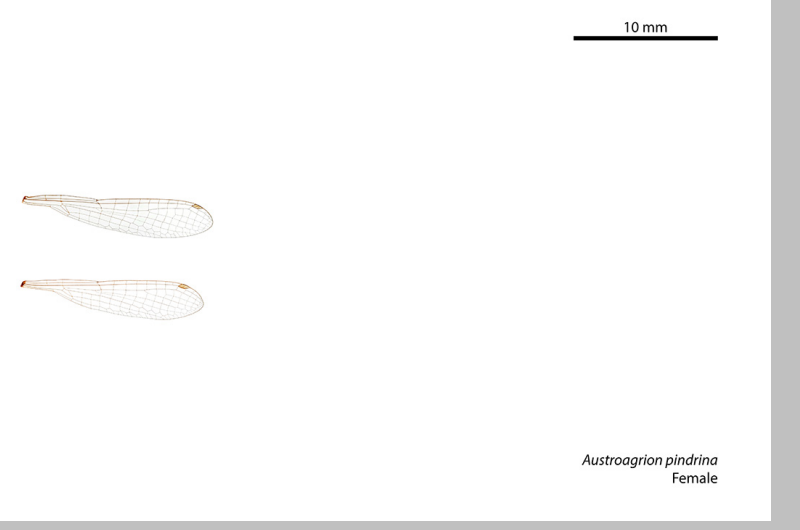

Austroagrion pindrina
Female

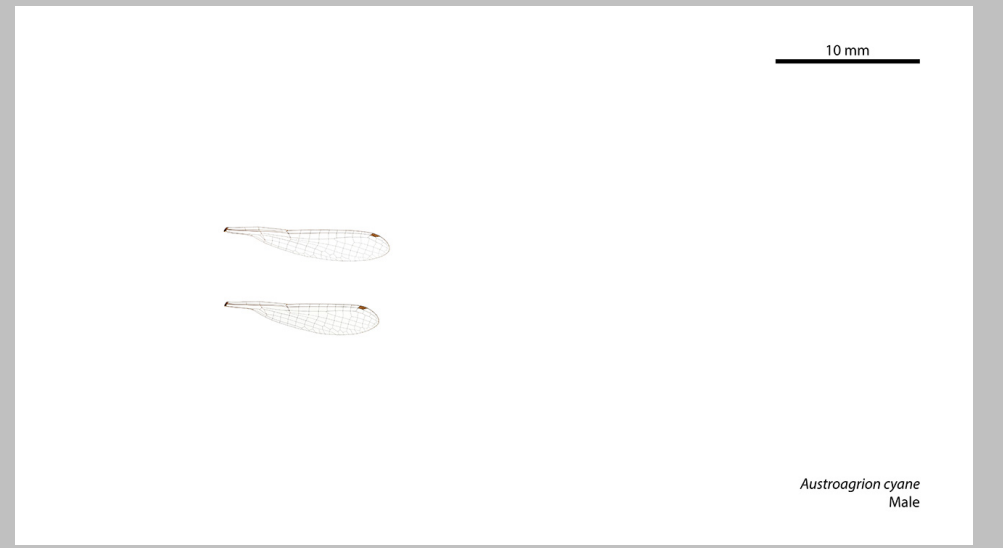

Austroagrion cyane male

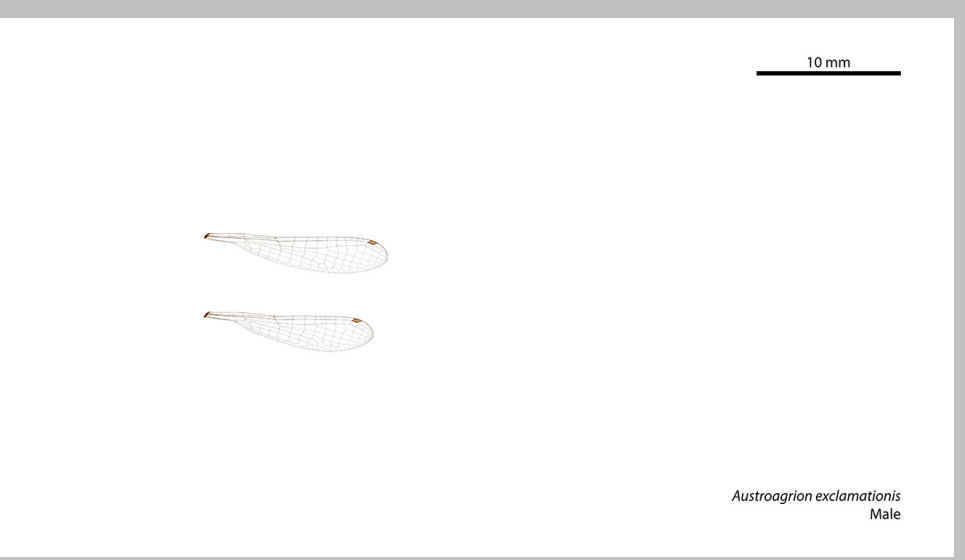

Austroagrion exclamationis male

Austroagrion pindrina female

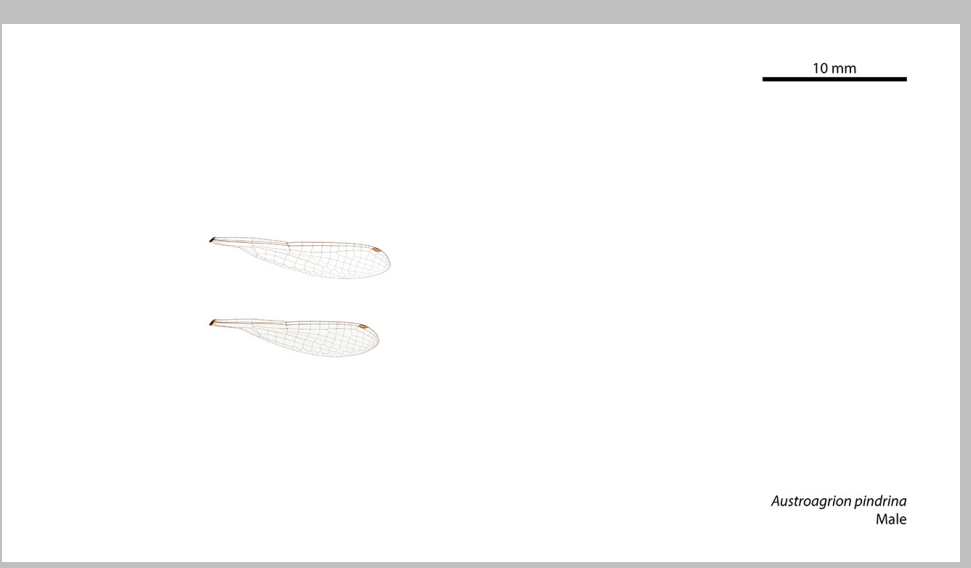

Austroagrion pindrina male
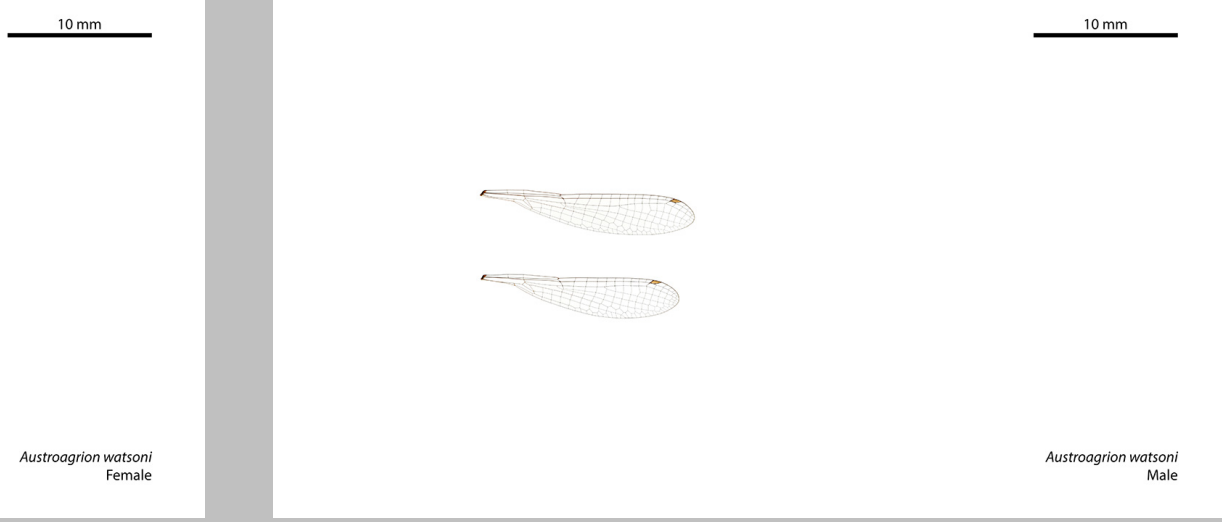

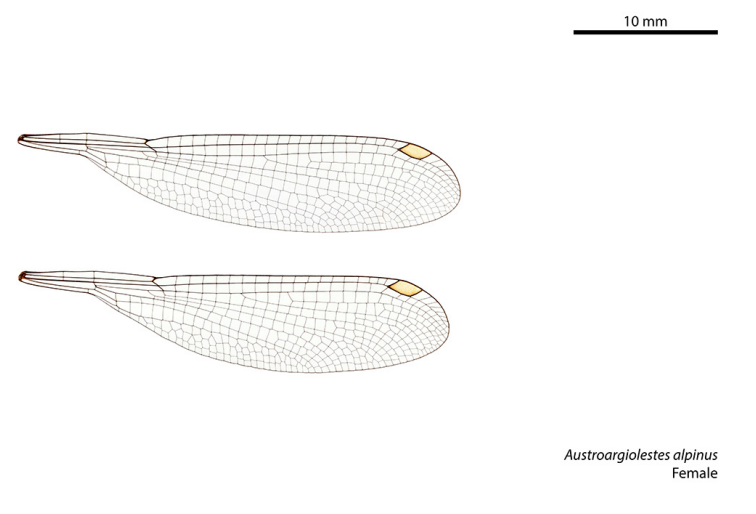

Austroargiolestes alpinus female

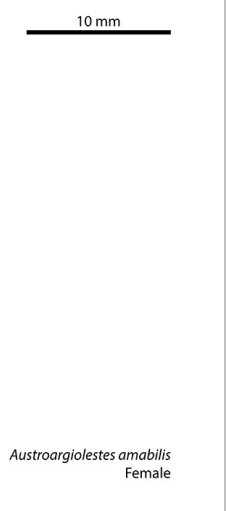

Austroargiolestes amabilis female

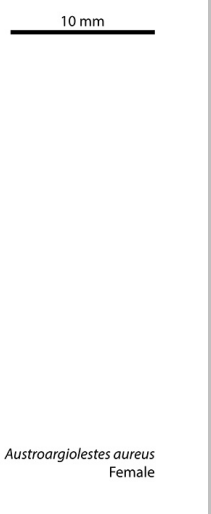

Austroargiolestes aureus female
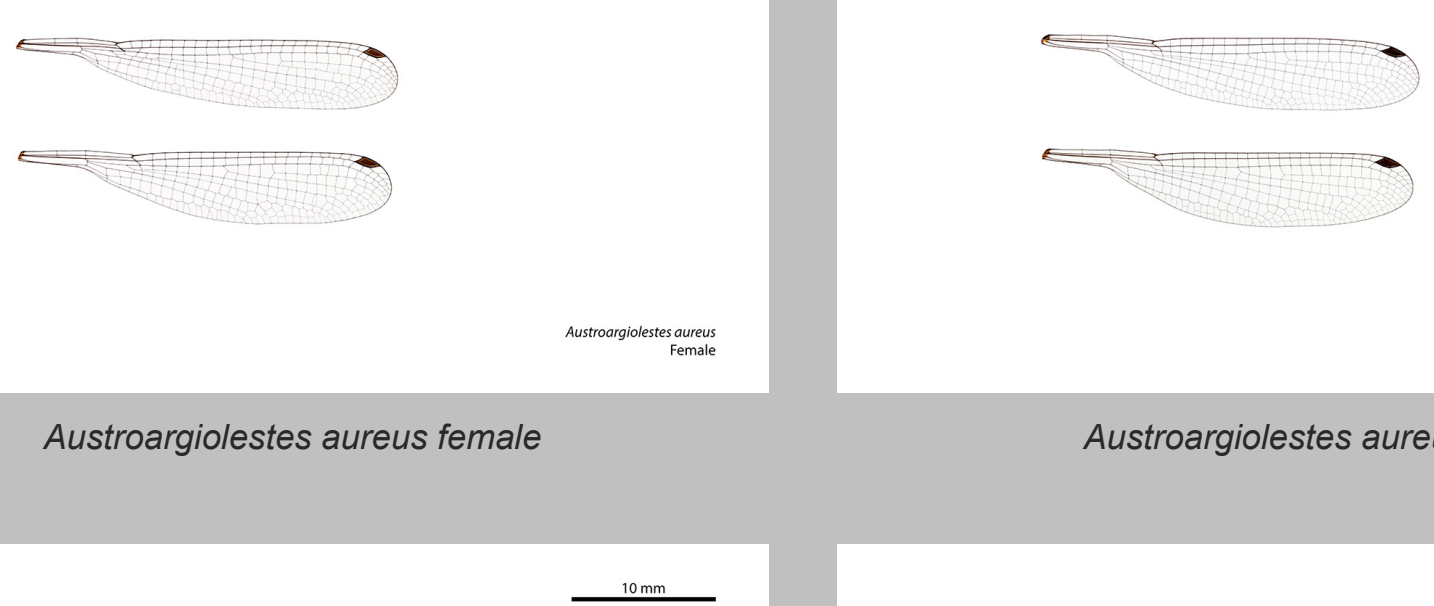

Austroargiolestes alpinus male
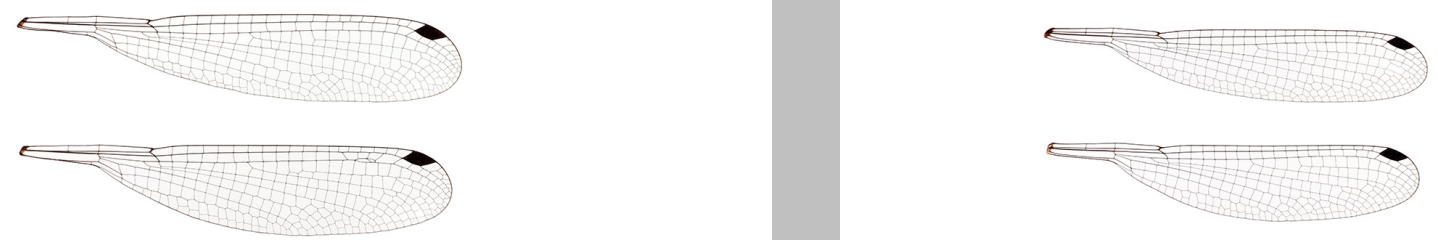

Austroargiolestes amabilis male

Austroargiolestes aureus male
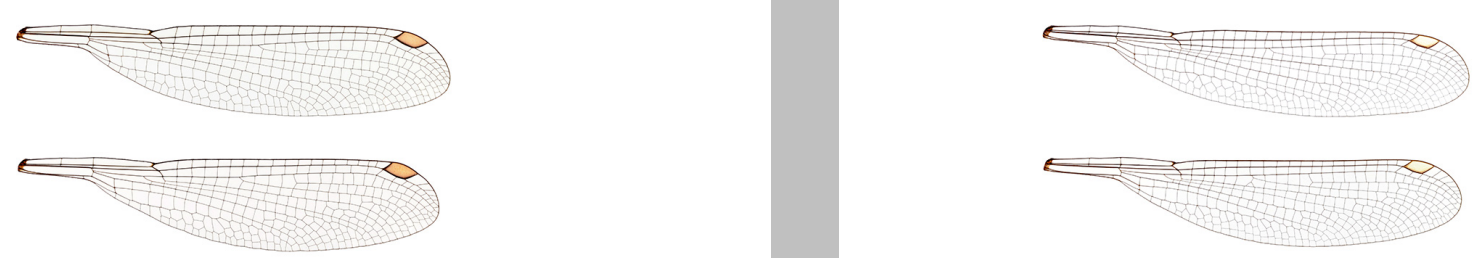


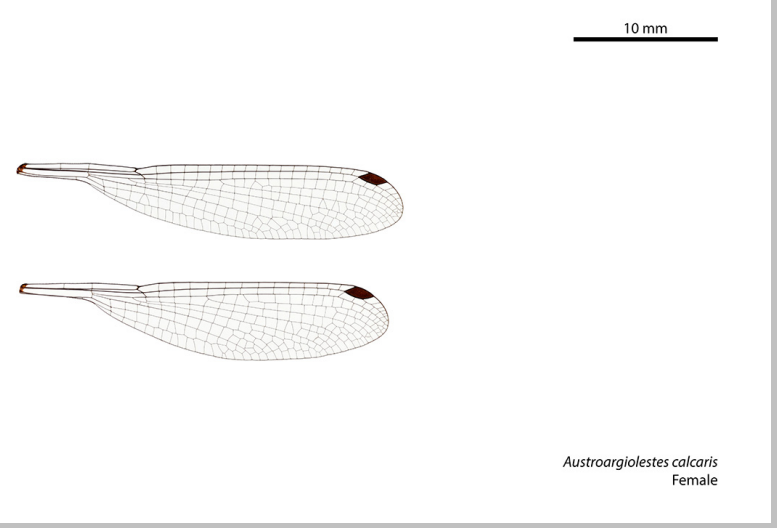

Austroargiolestes calcaris female

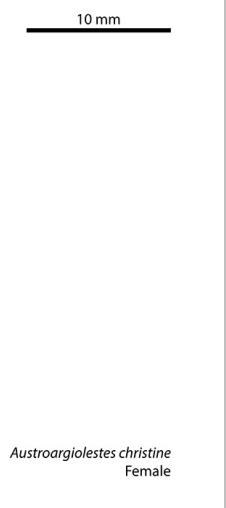

Austroargiolestes christine female
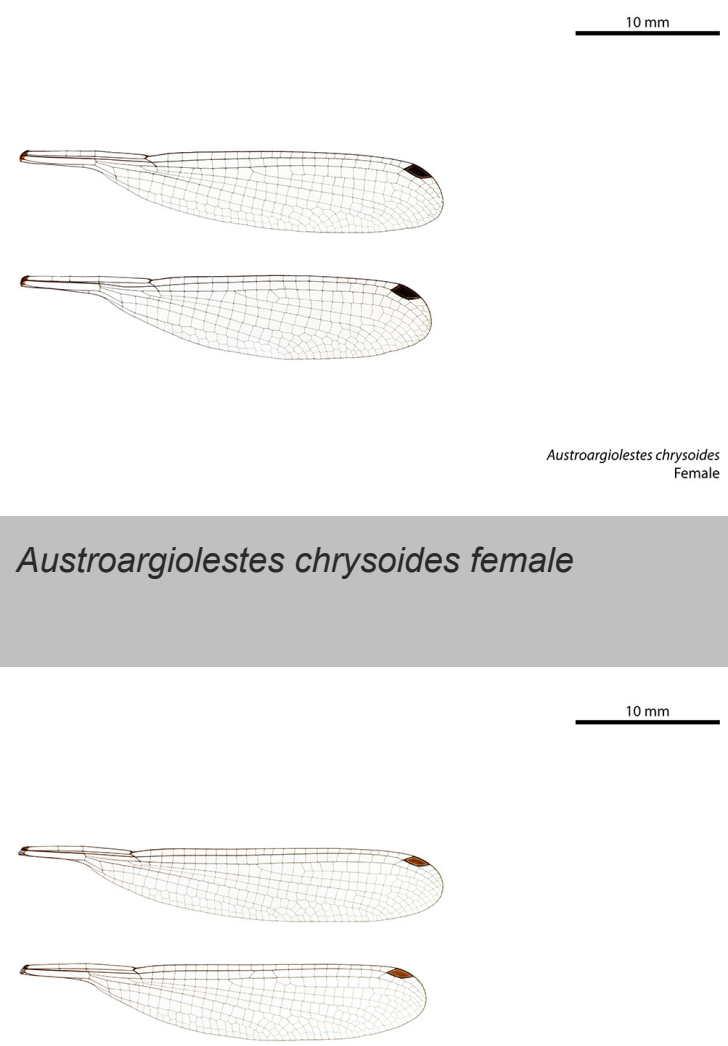
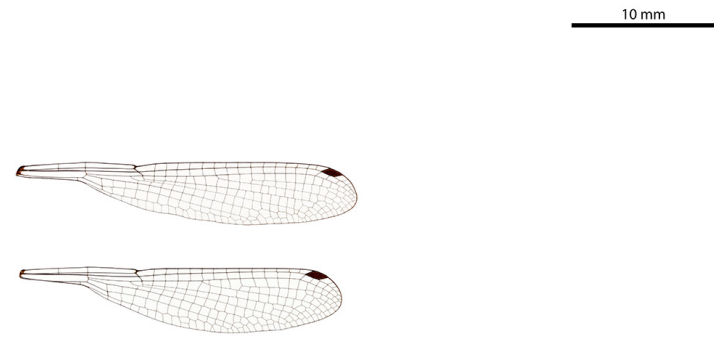

Austroargiolestes calcaris male

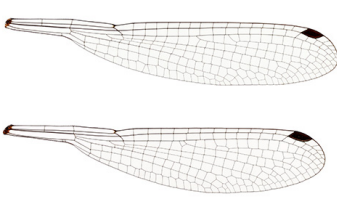

Austroargiolestes christine male
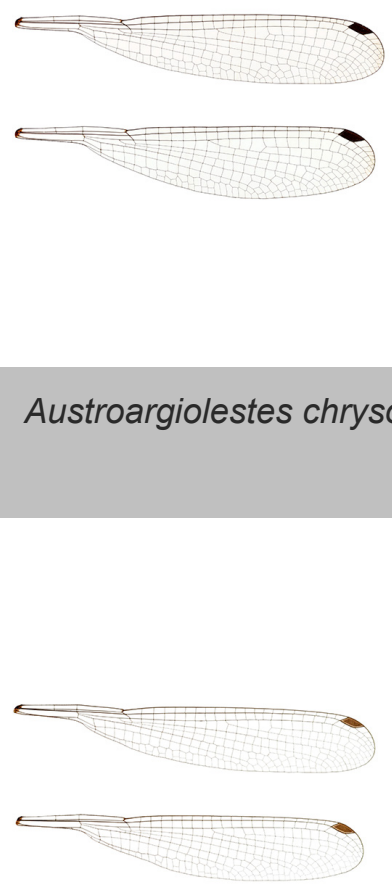


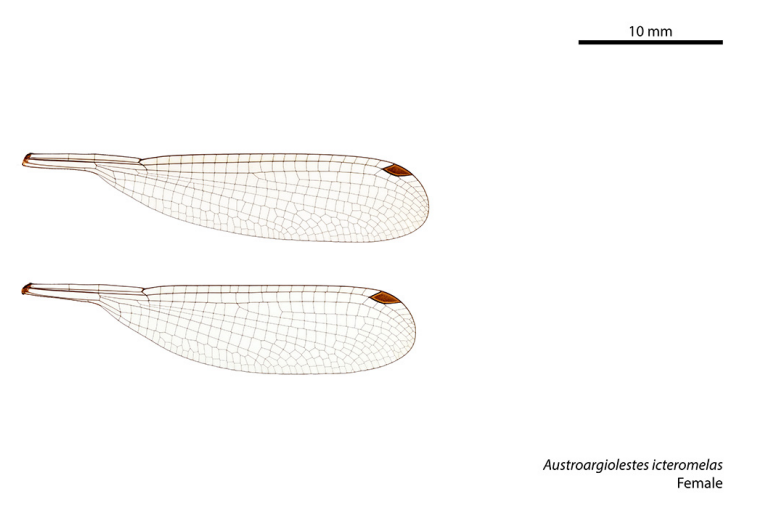

Austroargiolestes icteromelas female

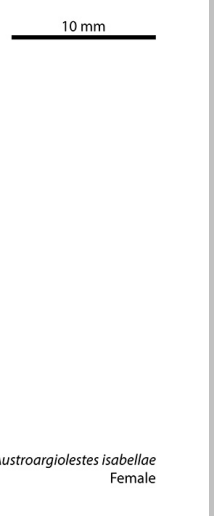

Austroargiolestes isabellae female

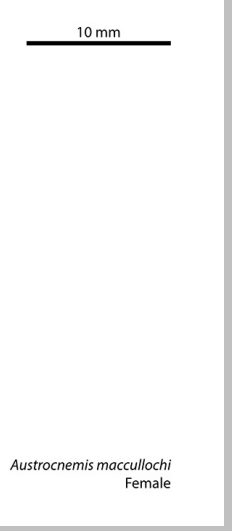

Austrocnemis maccullochi female

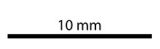

Austrocnemis obscura 


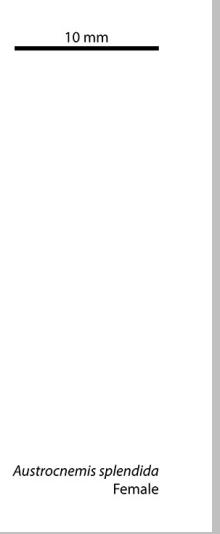

Austrocnemis splendida female

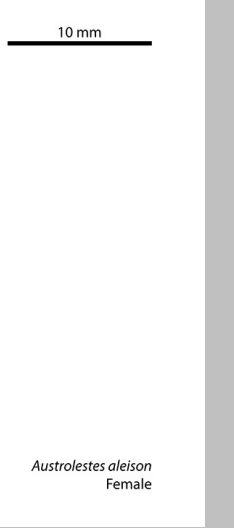

Austrolestes aleison female

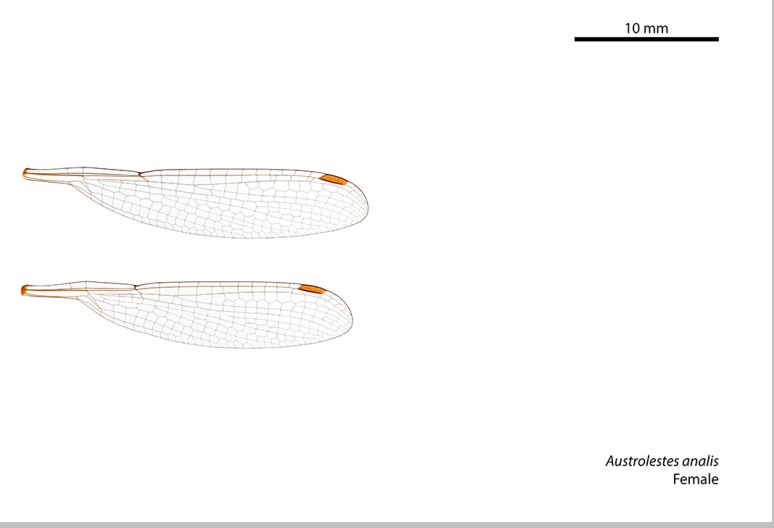

Austrolestes analis female

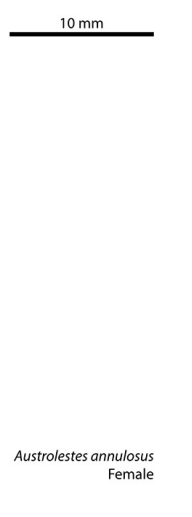

Austrolestes annulosus female

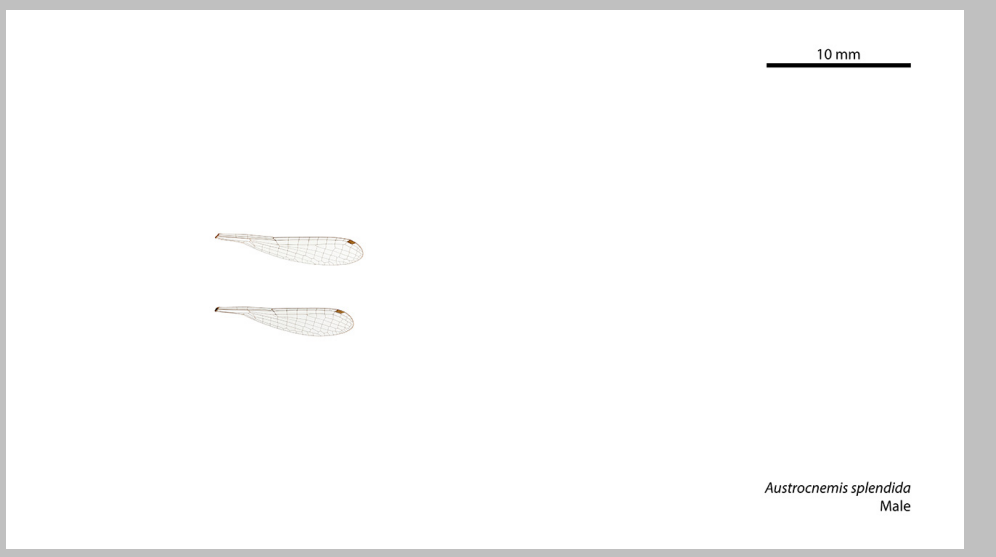

Austrocnemis splendida male

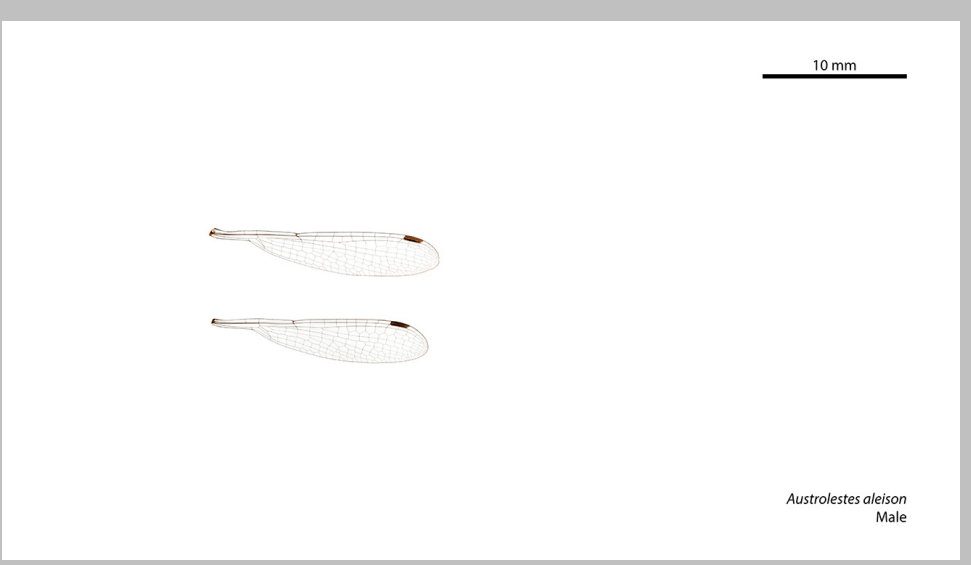

Austrolestes aleison male

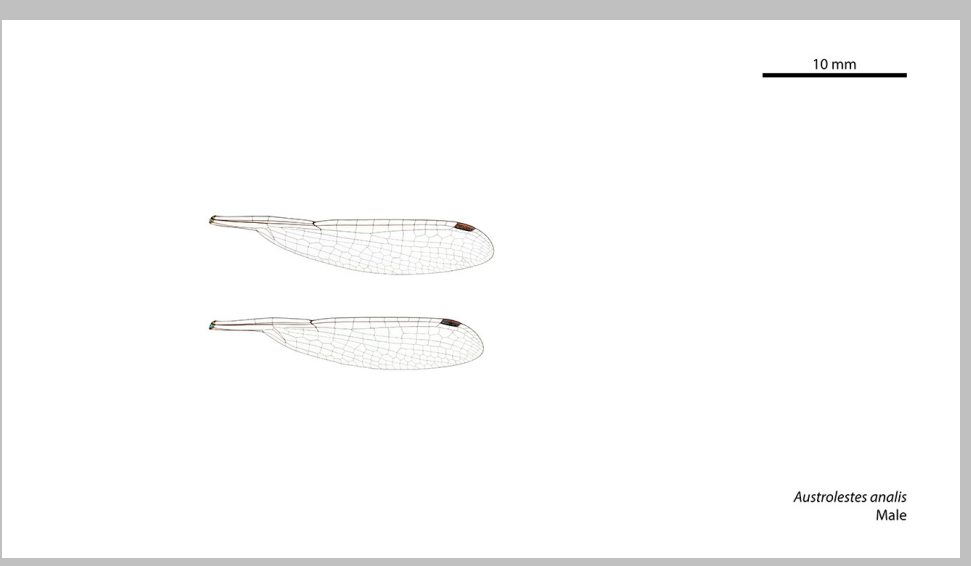

Austrolestes analis male

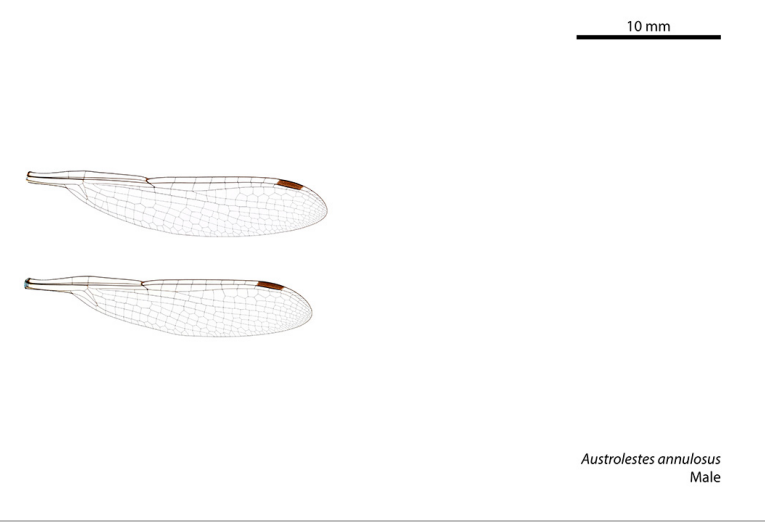

Austrolestes annulosus male 


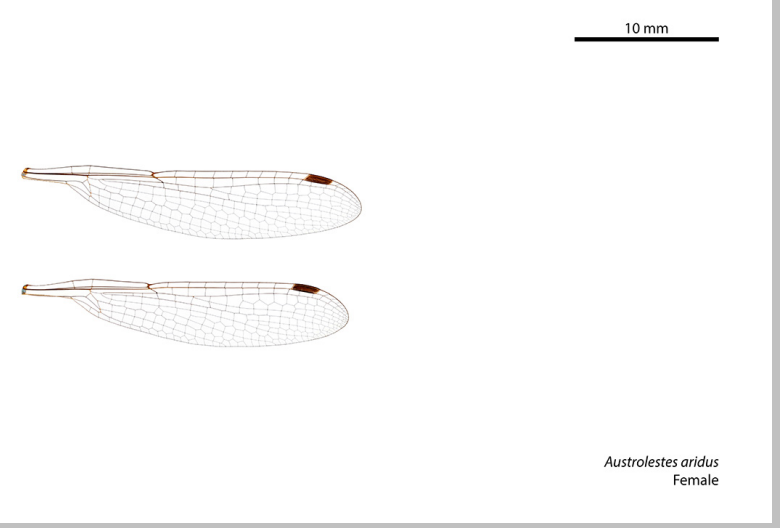

Austrolestes aridus female

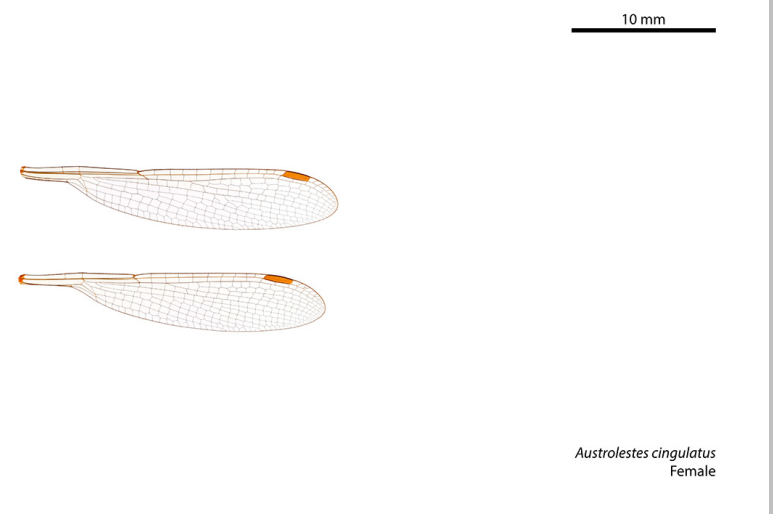

Austrolestes cingulatus female

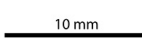

Austrolestes insularis

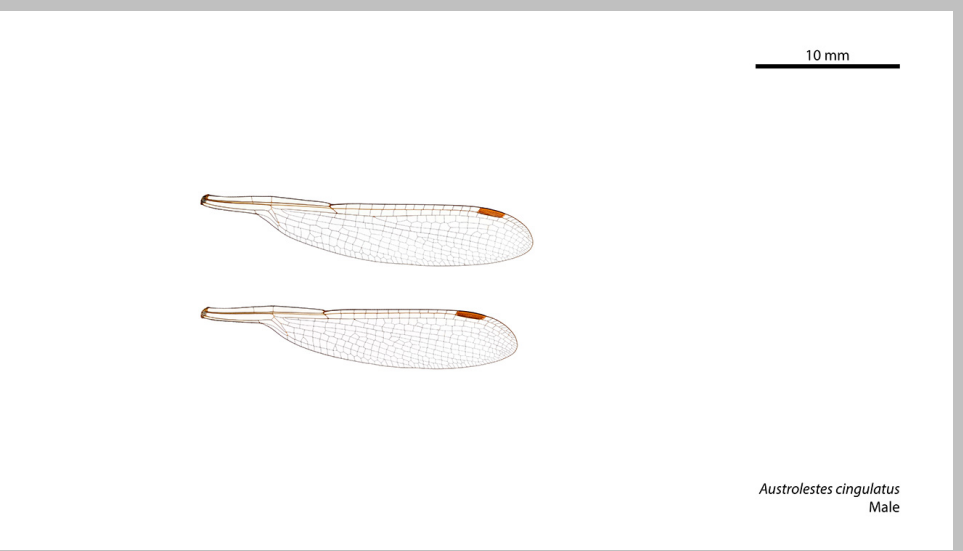

Austrolestes cingulatus male

Austrolestes aridus male
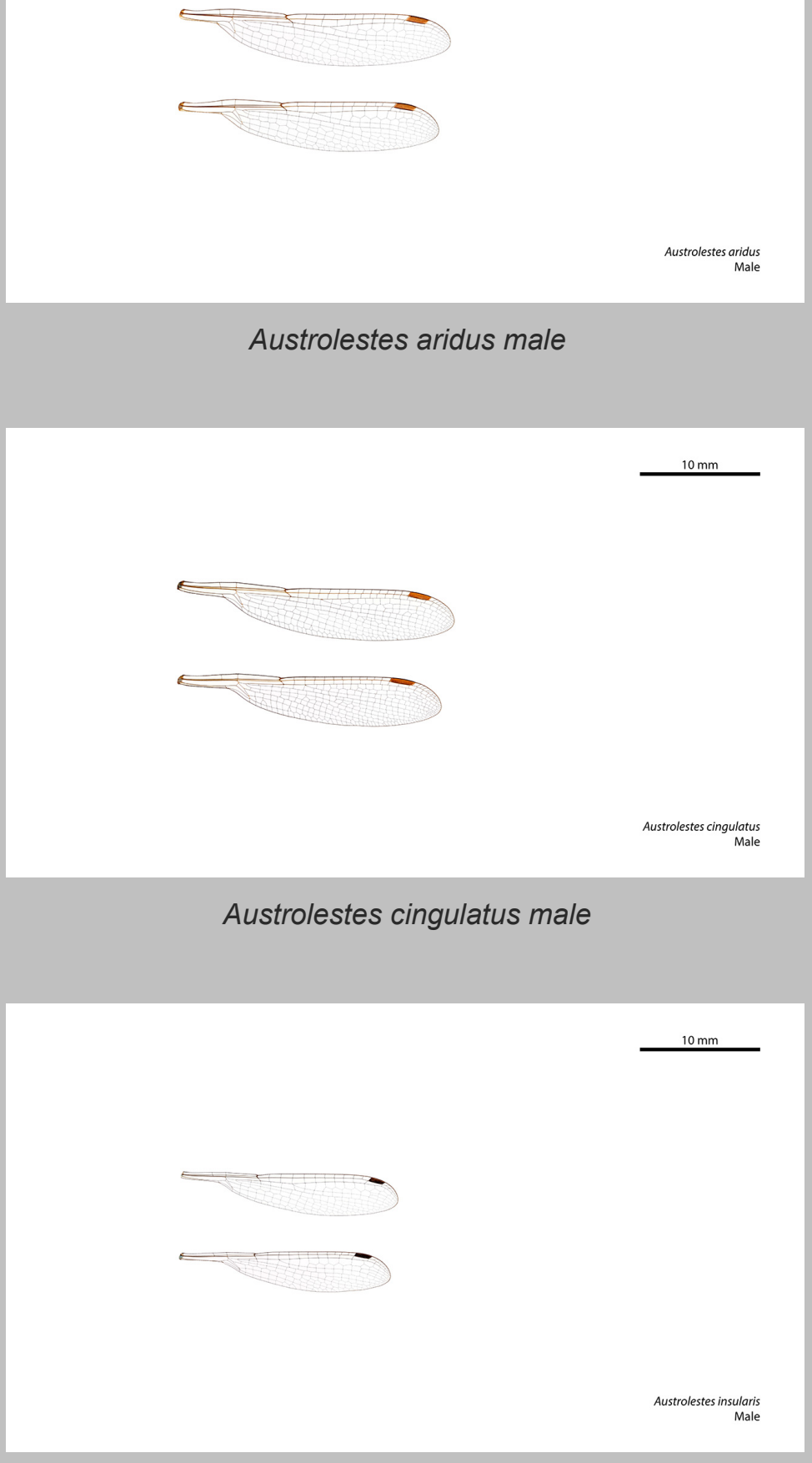

Austrolestes insularis male

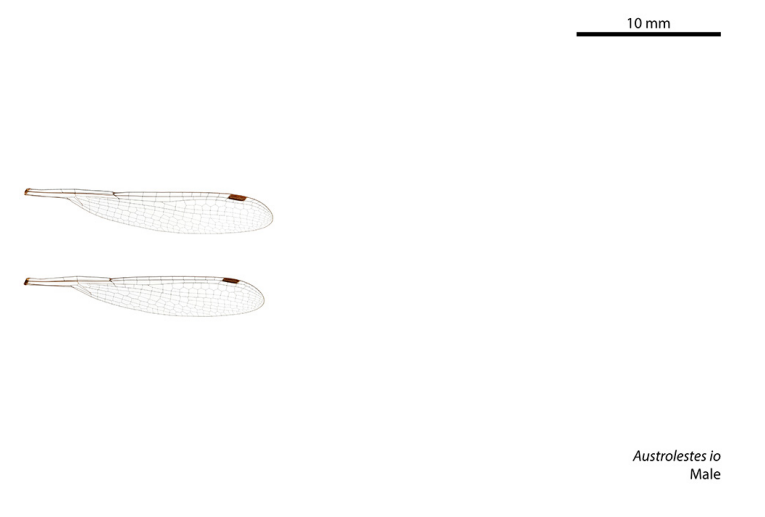

Austrolestes io male 


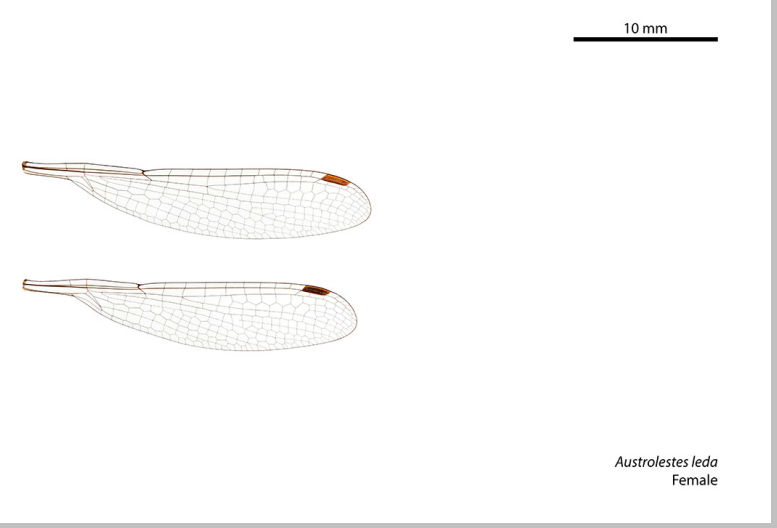

Austrolestes leda female

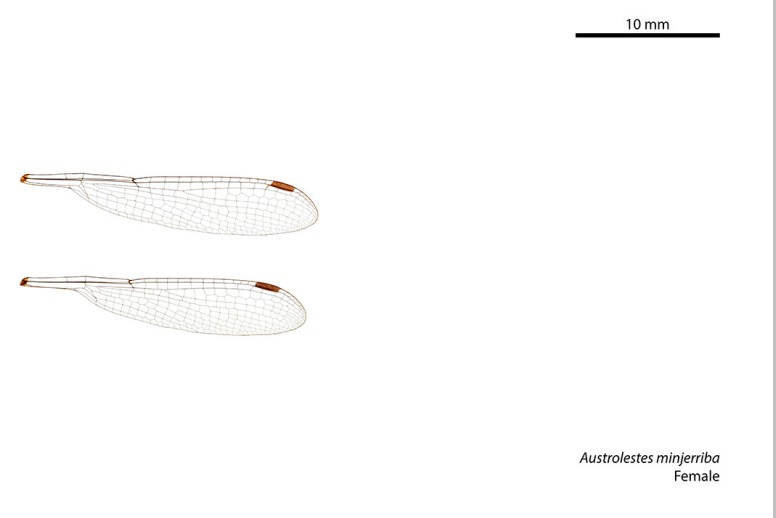

Austrolestes minjerriba female
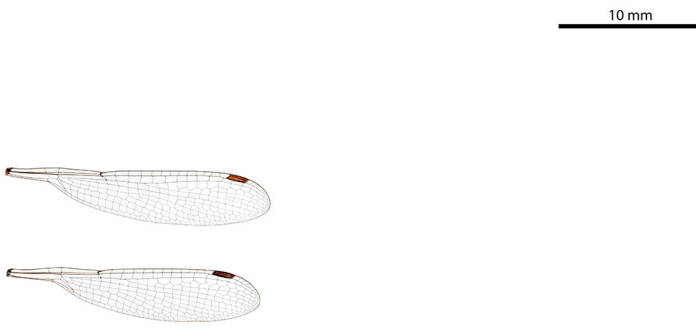

Austrolestes psyche

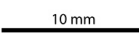

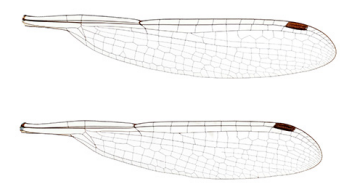

Austrolestes leda male

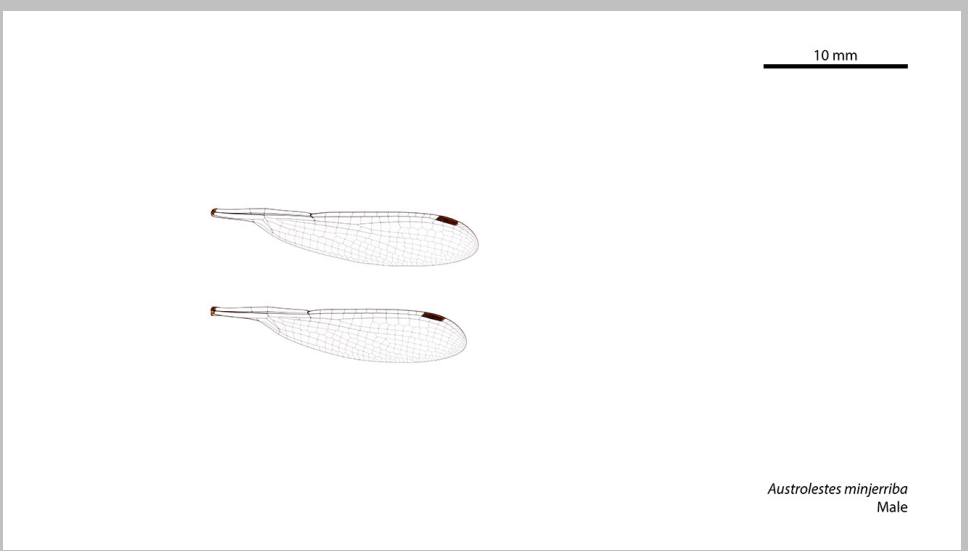

Austrolestes minjerriba male

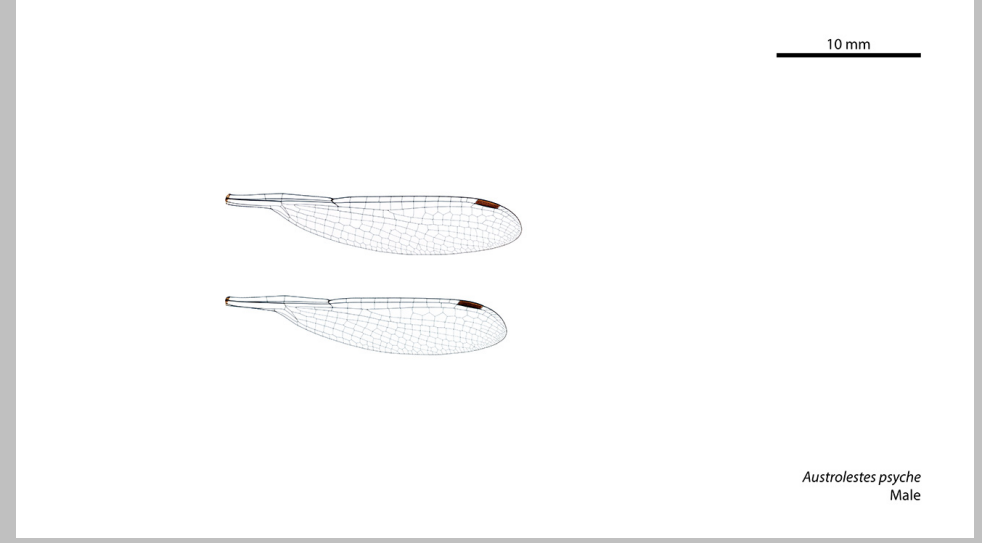

Austrolestes psyche male 


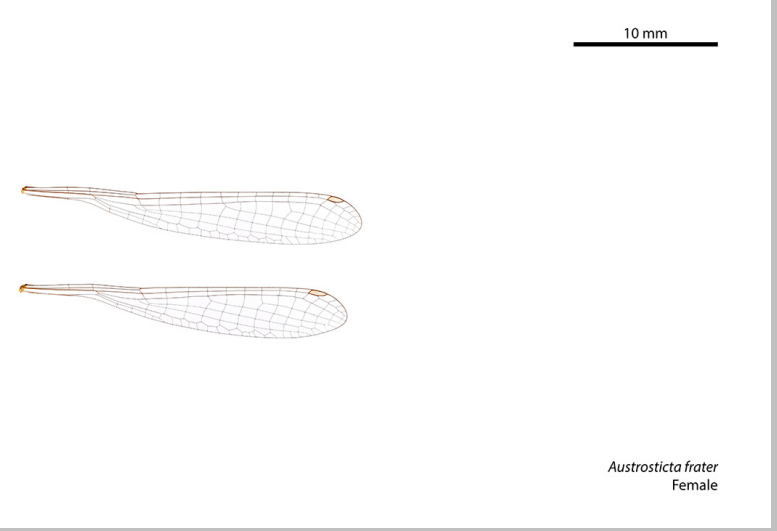

Austrosticta frater female

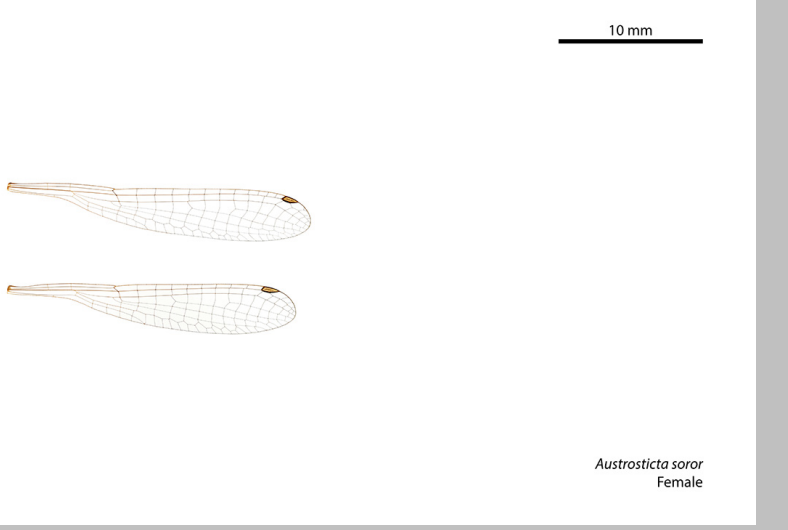

Austrosticta soror female

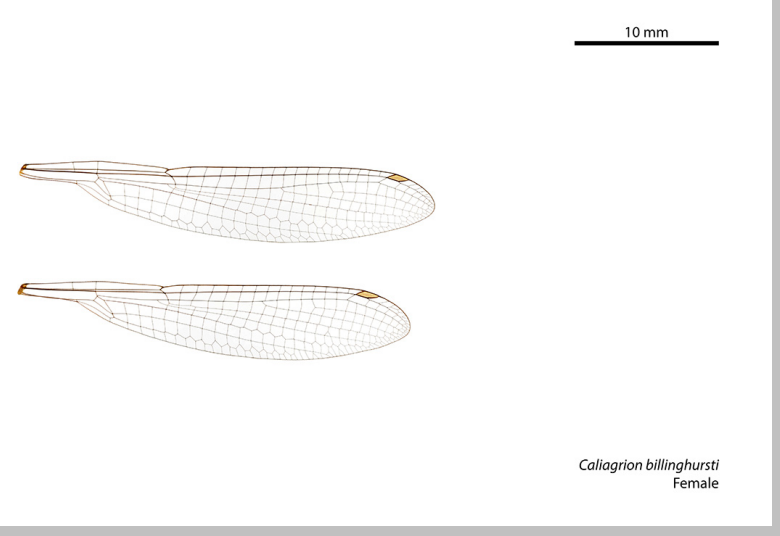

Caliagrion billinghursti female

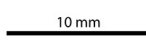

Ceriagrion aeruginosum

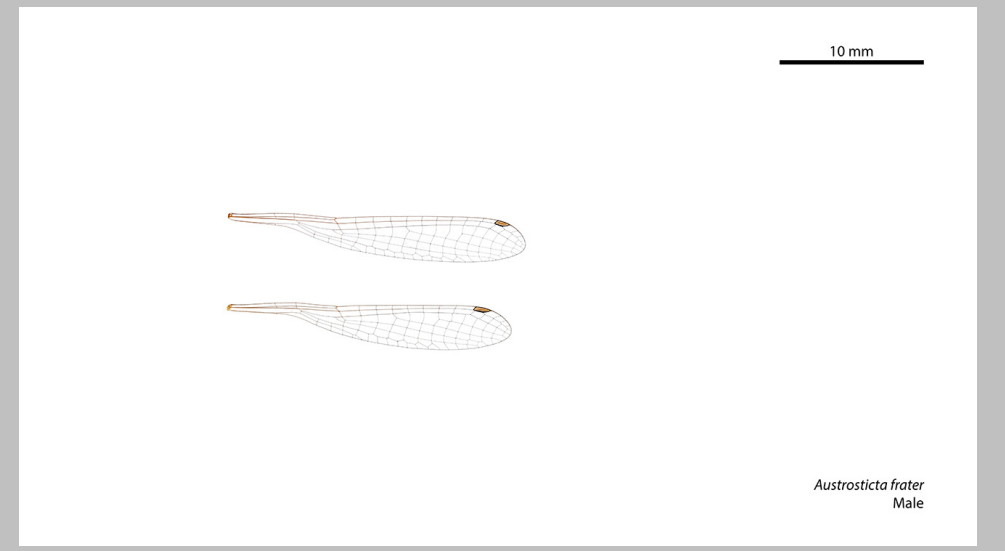

Austrosticta frater male

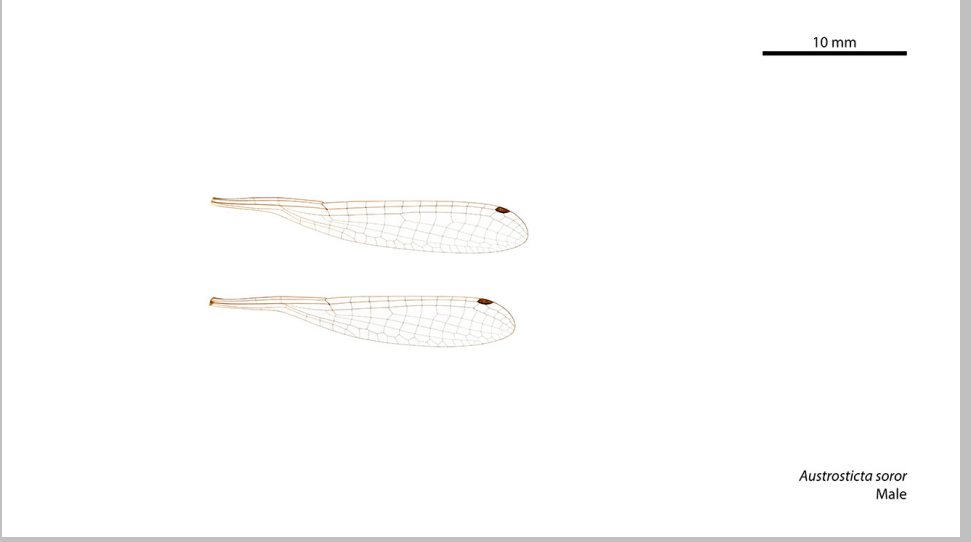

Austrosticta soror male

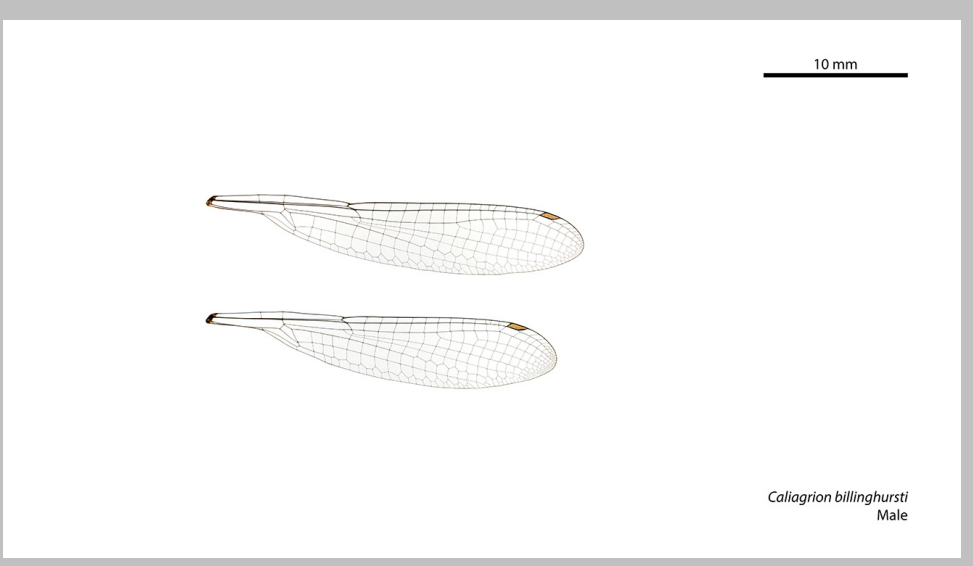

Caliagrion billinghursti male

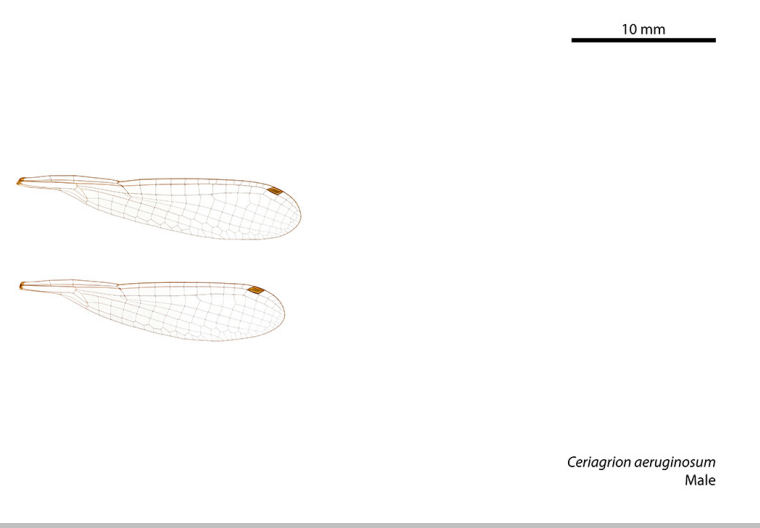

Ceriagrion aeruginosum male 


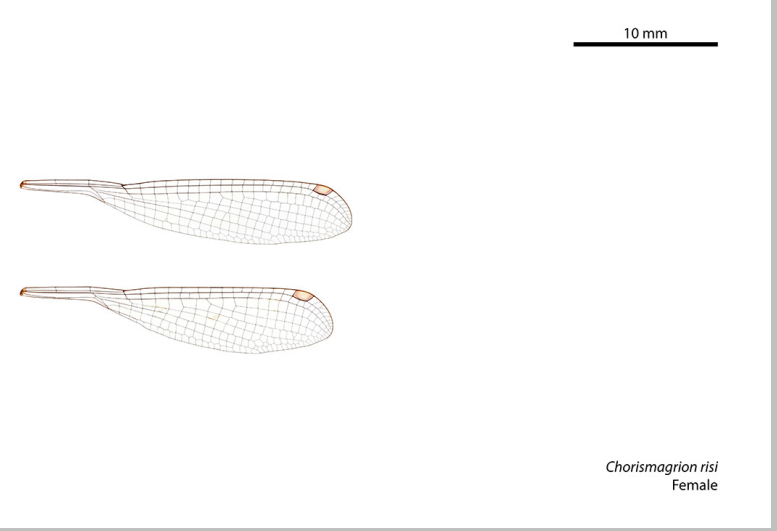

Chorismagrion risi female

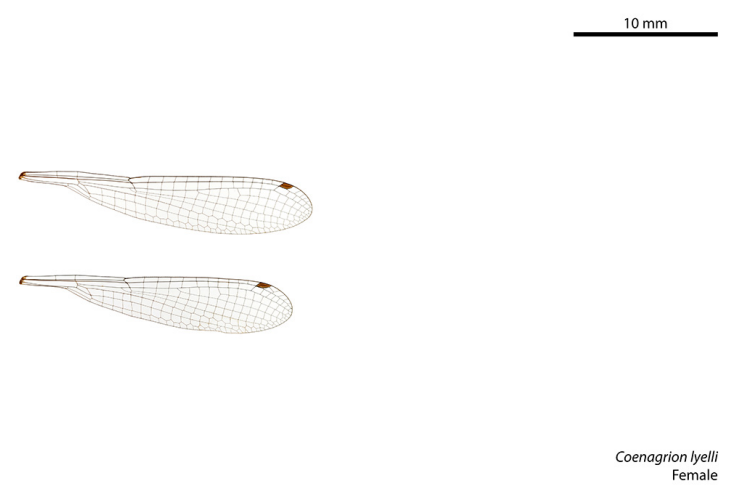

Coenagrion lyelli female

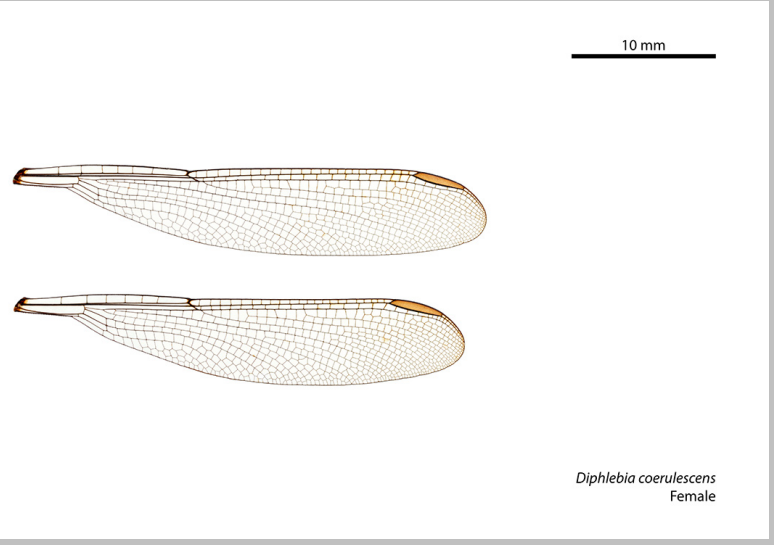

Diphlebia coerulescens female
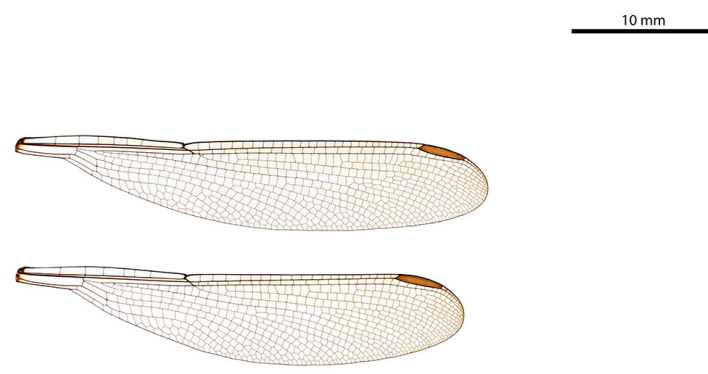

Diphlebia euphoeoides

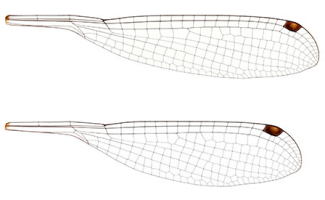

Chorismagrion risi male

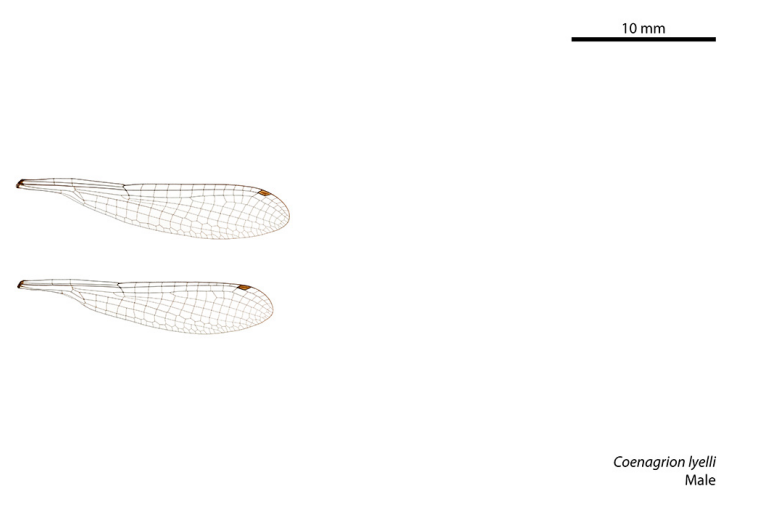

Coenagrion lyelli male

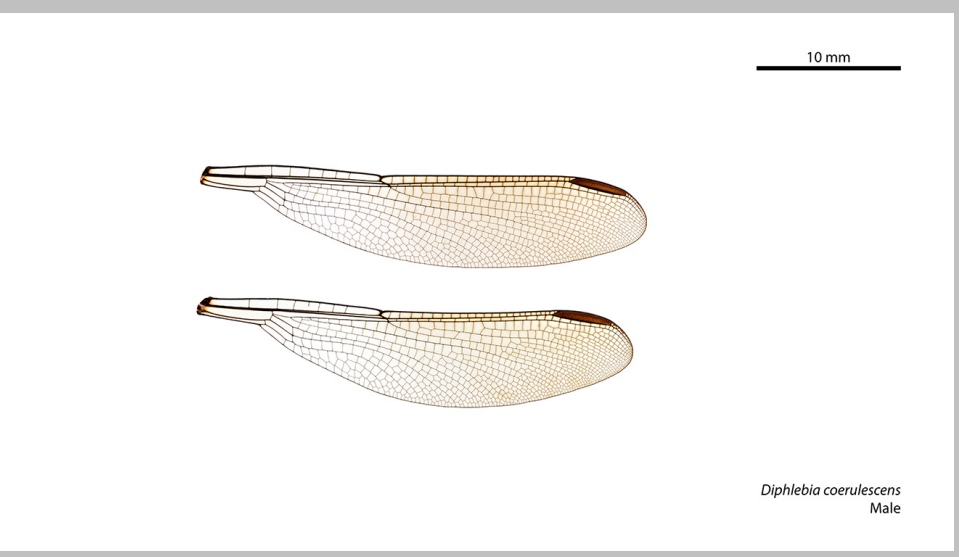

Diphlebia coerulescens male

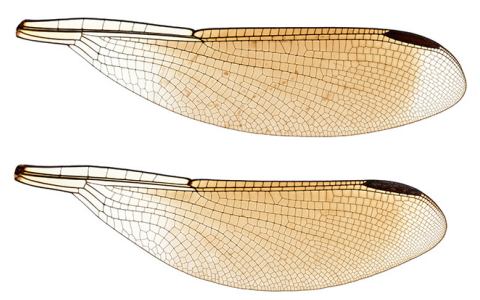

Diphlebia euphoeoides 


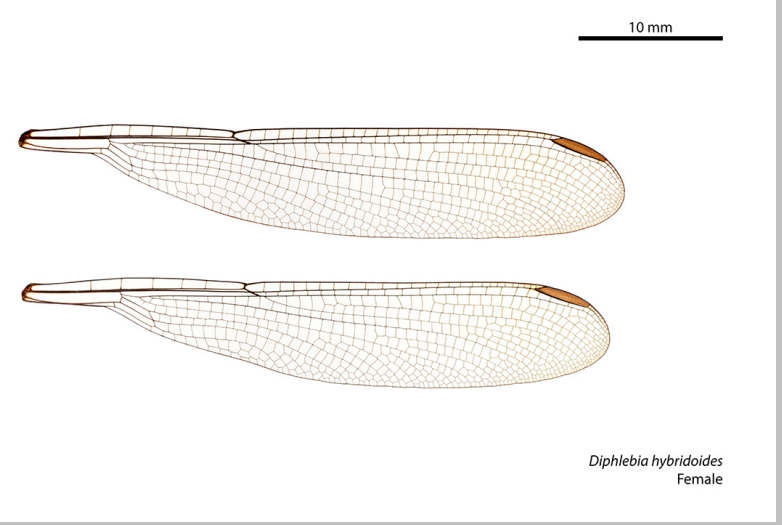

Diphlebia hybridoides female

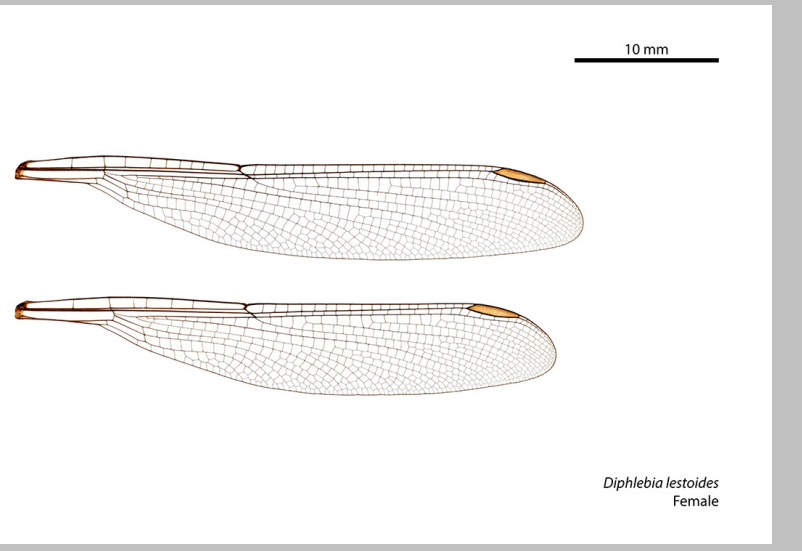

Diphlebia lestoides female

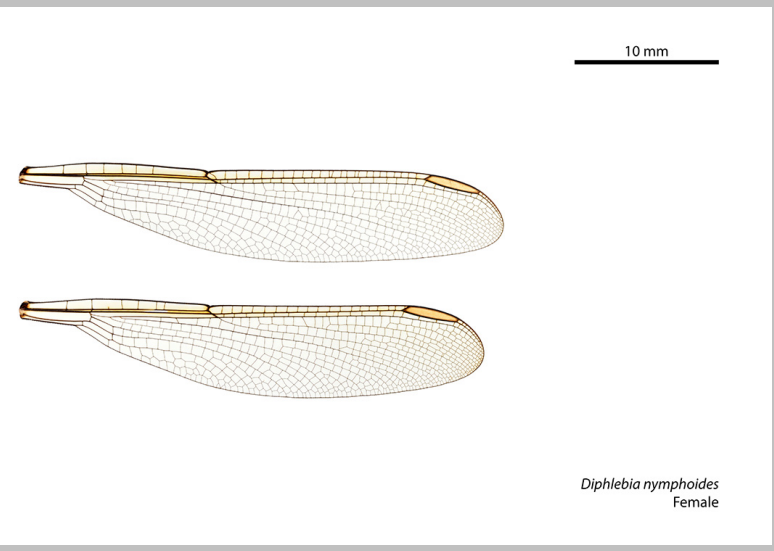

Diphlebia nymphoides female

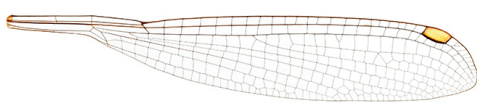

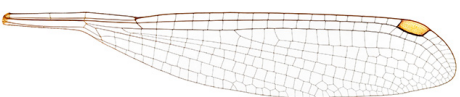
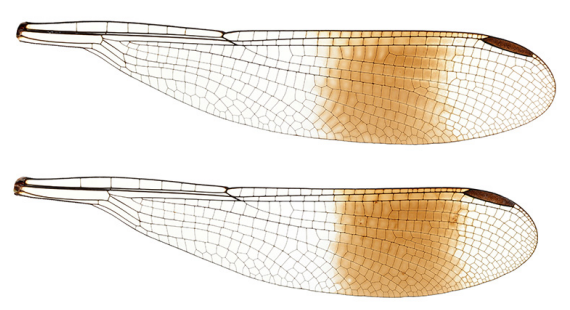

Diphlebia hybridoides
Male

Diphlebia hybridoides male
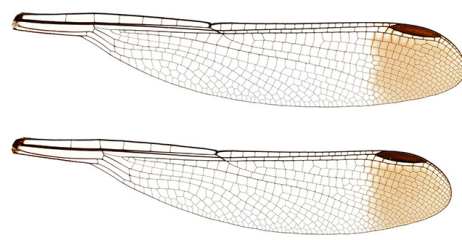

Diphlebia lestoides
Male

Diphlebia lestoides male

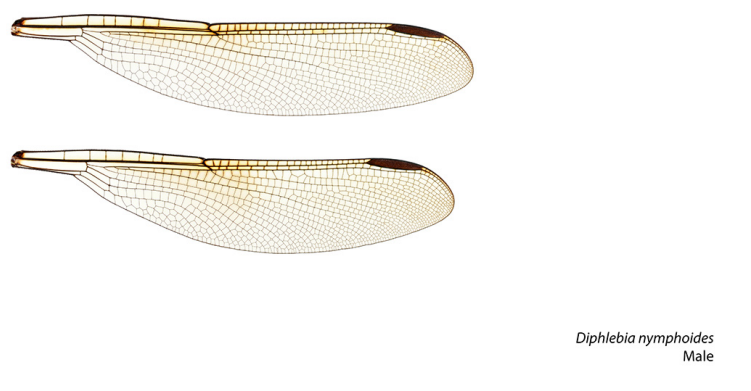

Diphlebia nymphoides male

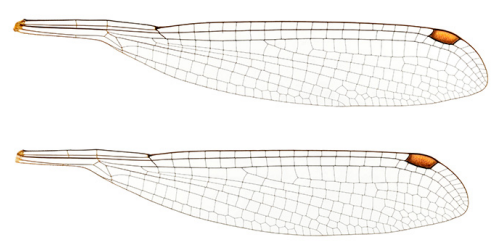




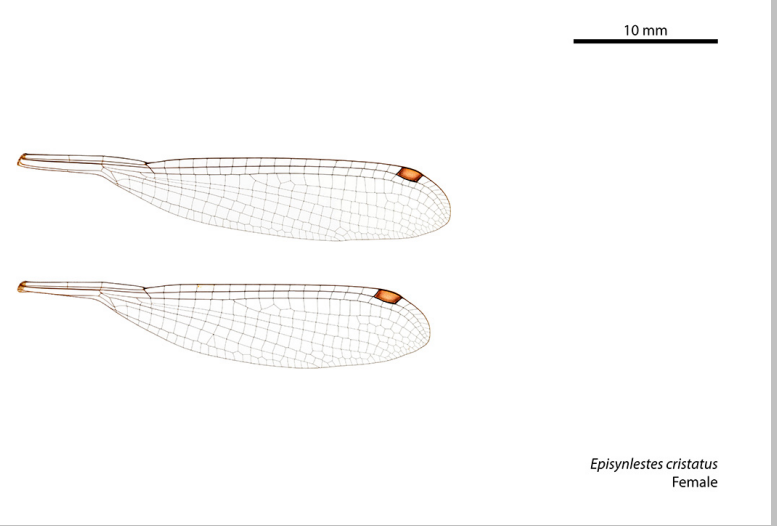

Episynlestes cristatus female

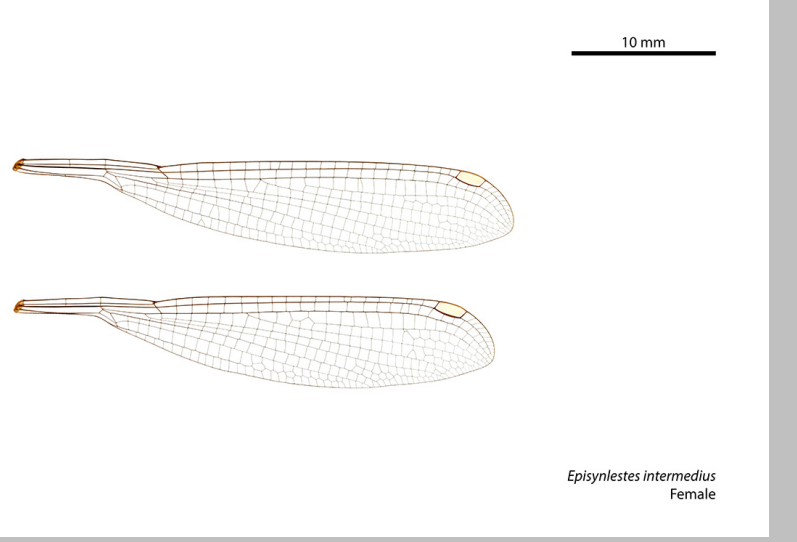

Episynlestes intermedius female

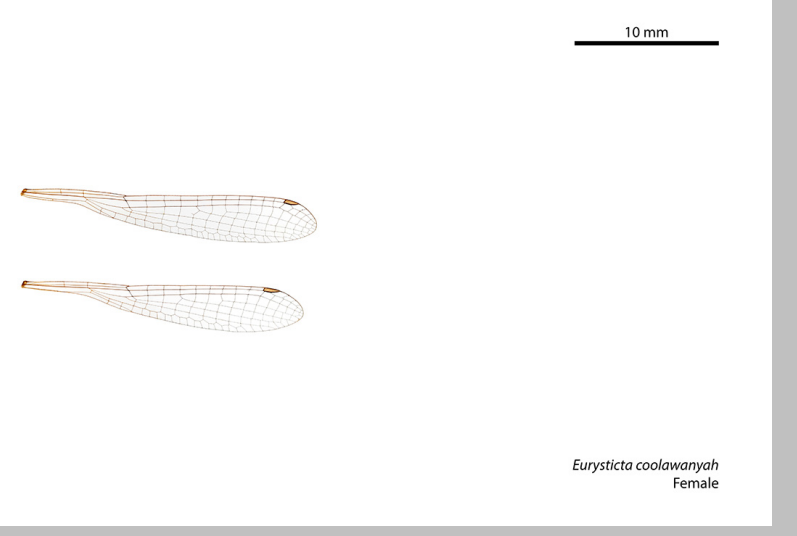

Eurysticta coolawanyah female

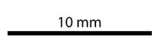

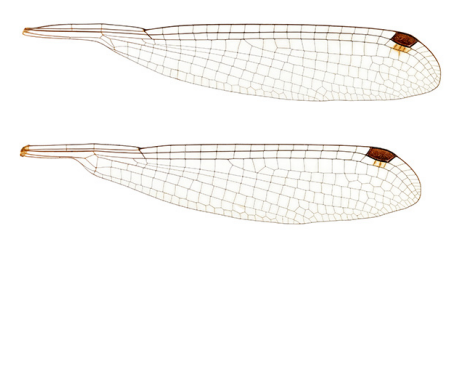

Episynlestes cristatus male

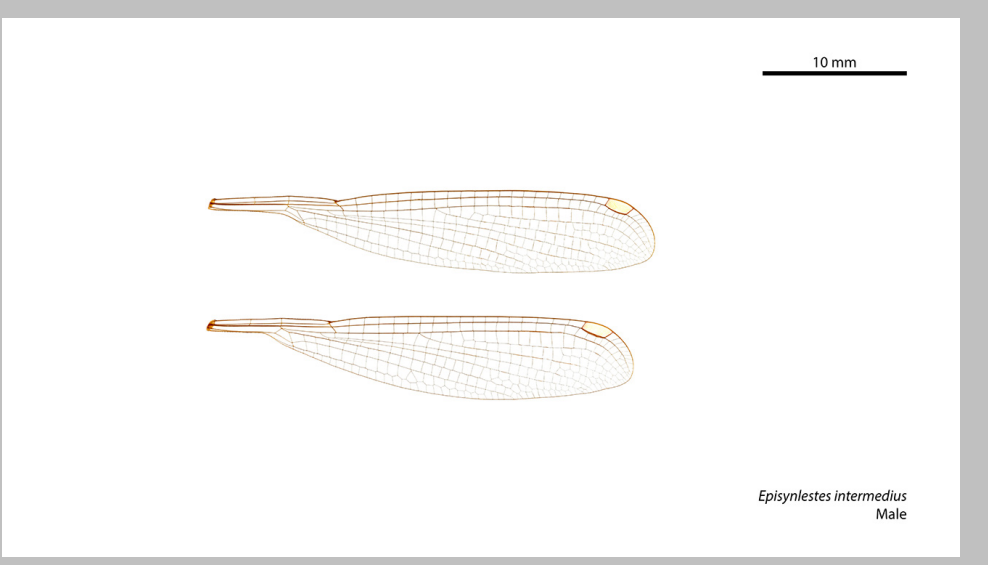

Episynlestes intermedius male

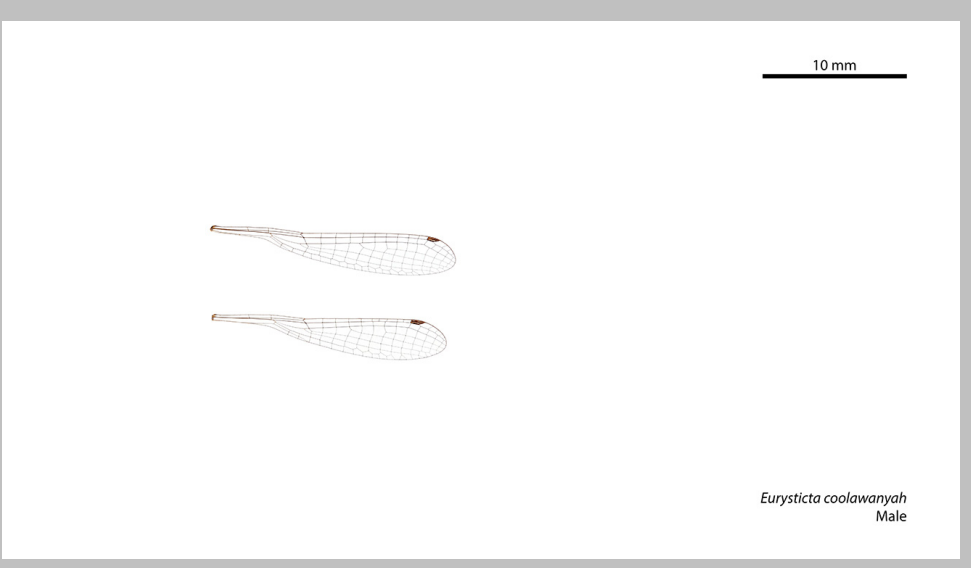

Eurysticta coolawanyah male 


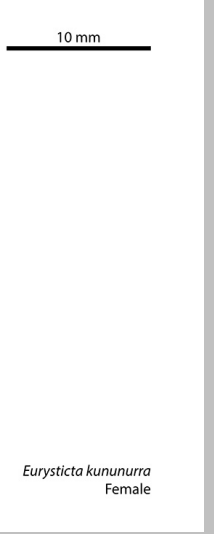

Eurysticta kununurra female

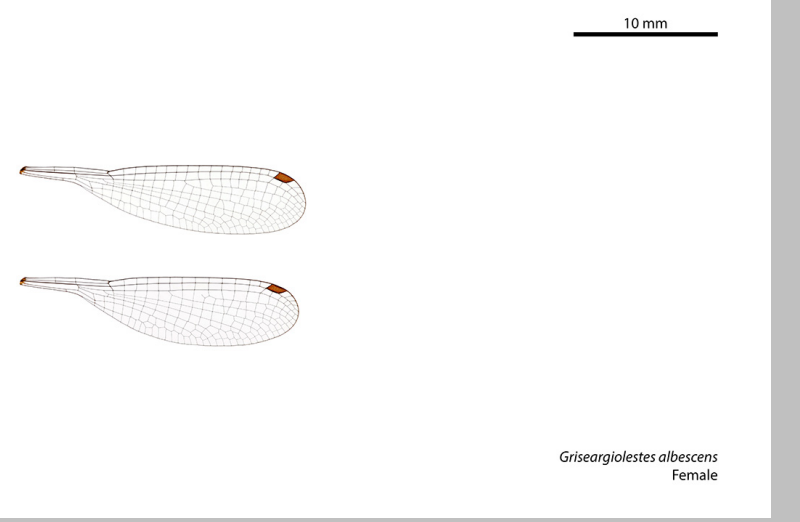

Griseargiolestes albescens female

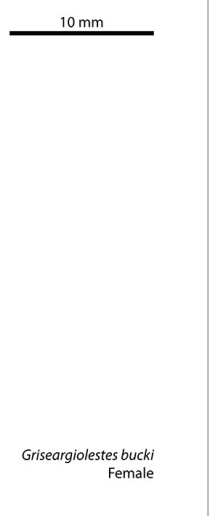

Griseargiolestes bucki female

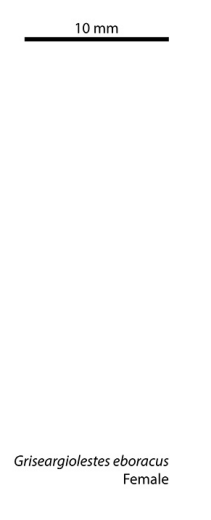

Griseargiolestes eboracus female
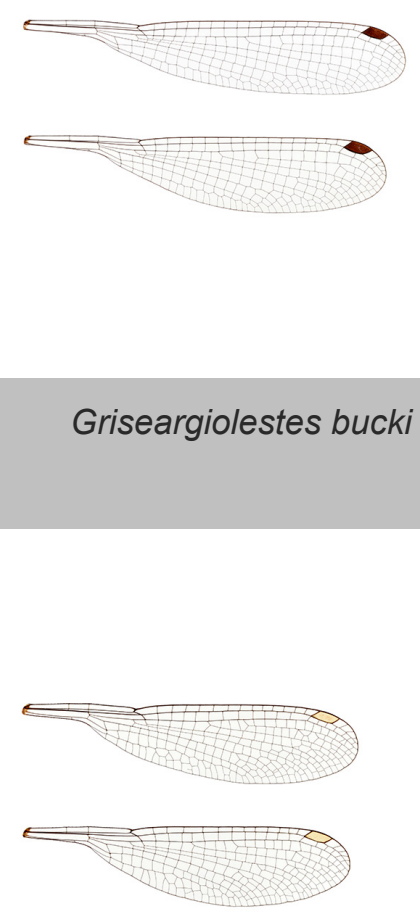

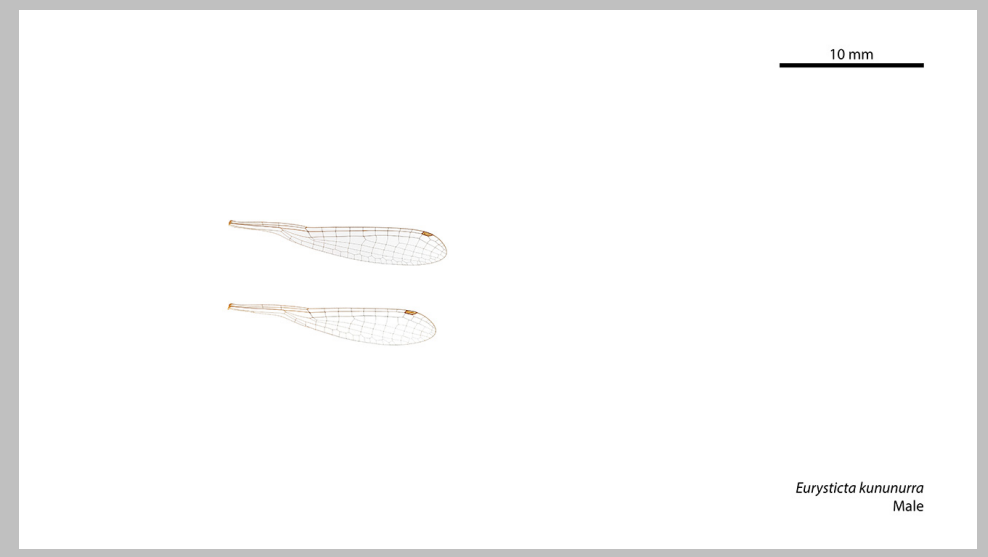

Eurysticta kununurra male

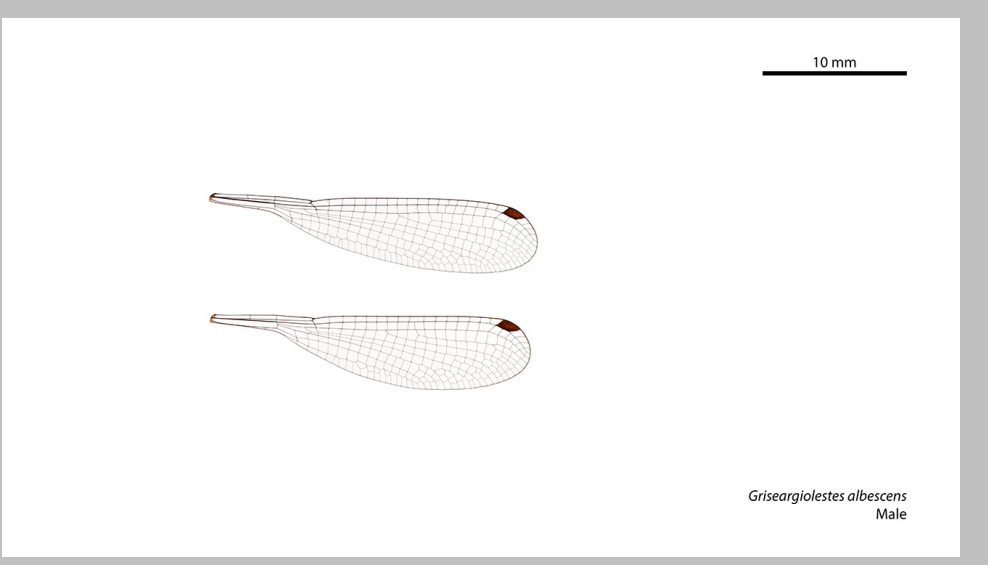

Griseargiolestes albescens male

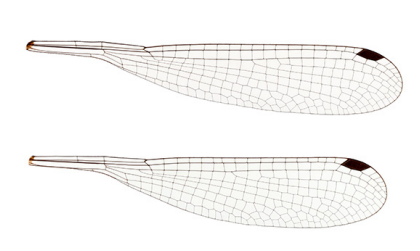

Griseargiolestes bucki
Male

Griseargiolestes bucki male

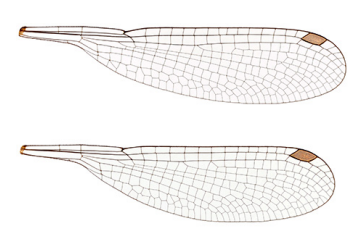




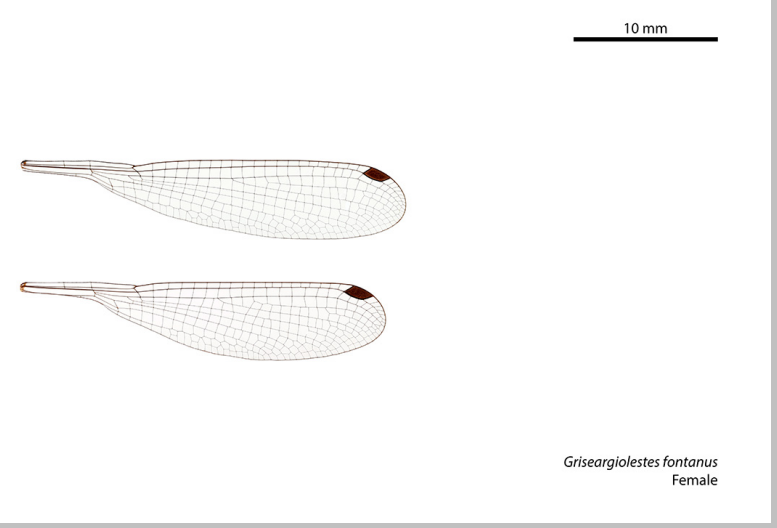

Griseargiolestes fontanus female

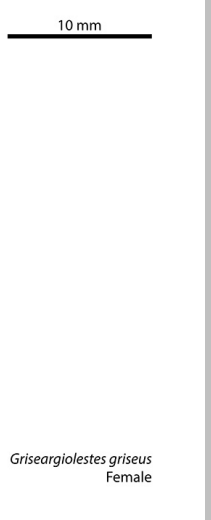

Griseargiolestes griseus female

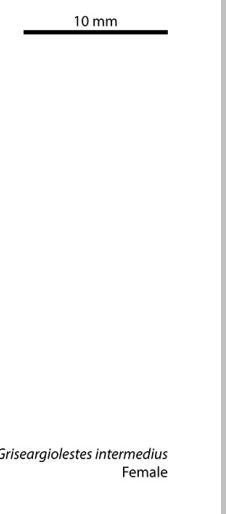

Griseargiolestes intermedius female

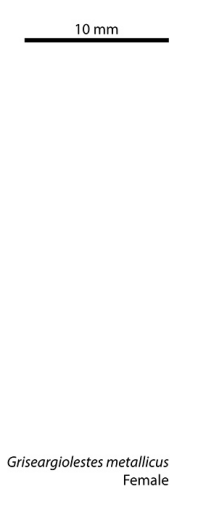

Griseargiolestes metallicus female

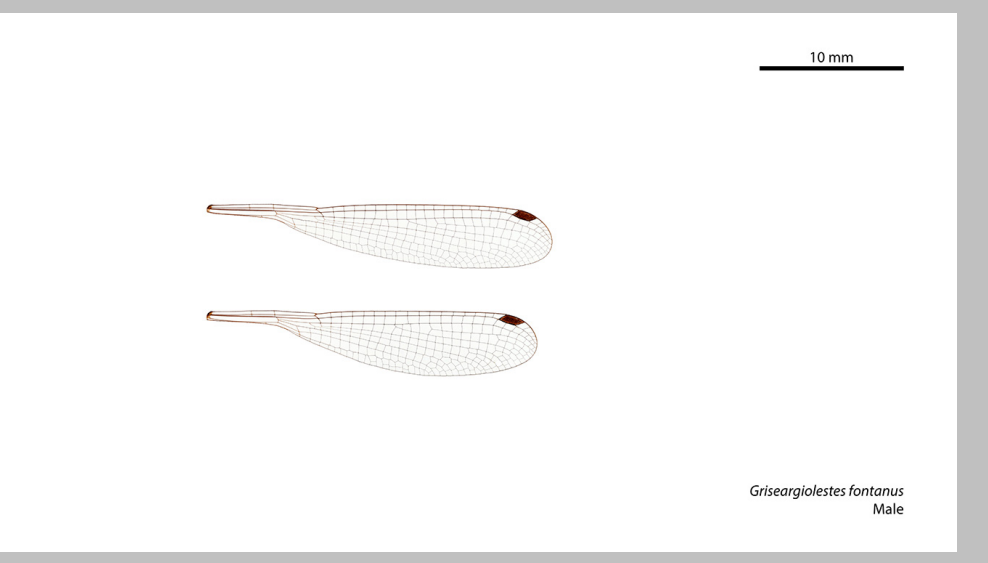

Griseargiolestes fontanus male

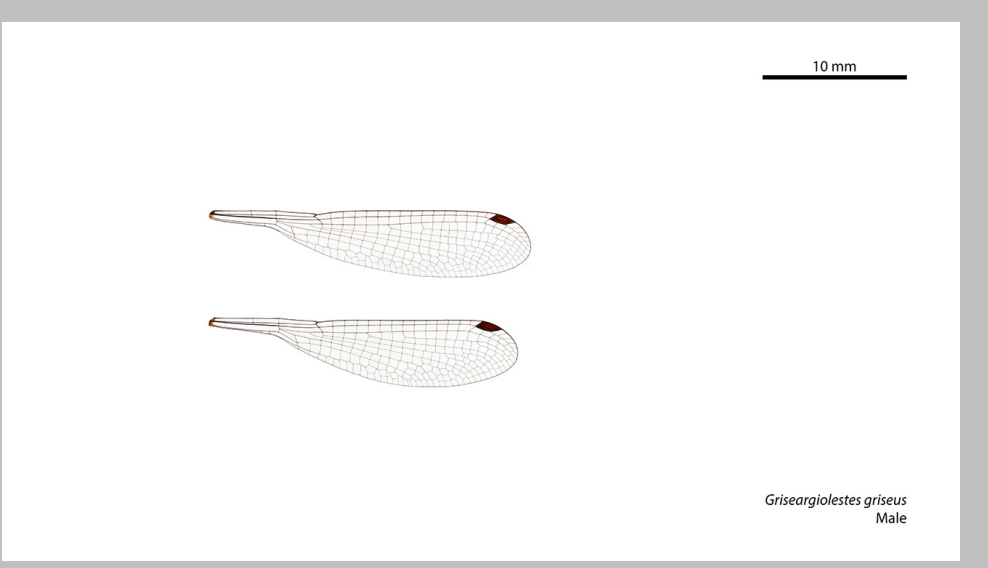

Griseargiolestes griseus male

Griseargiolestes intermedius
Male

Griseargiolestes intermedius male

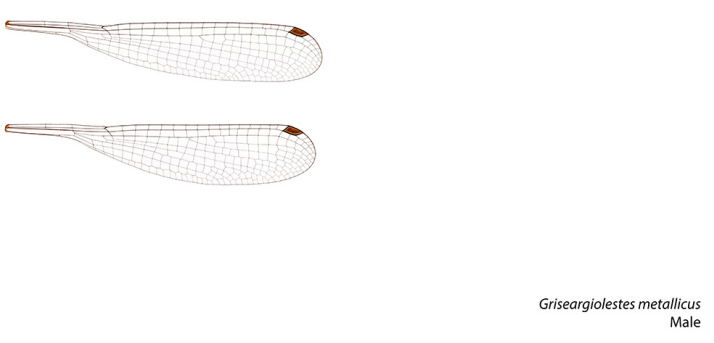

Griseargiolestes metallicus male 


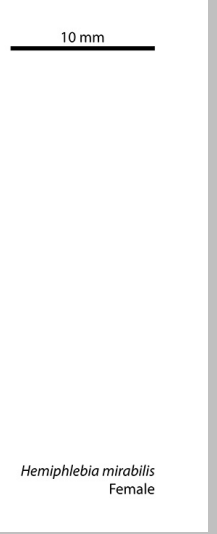

Hemiphlebia mirabilis female

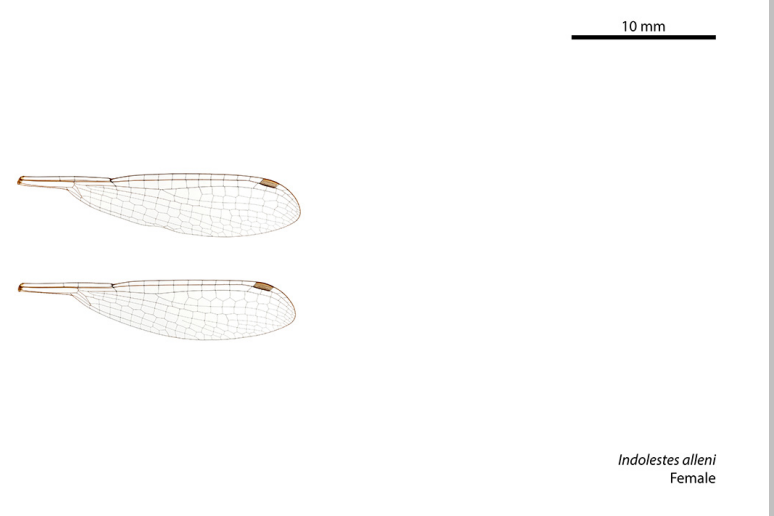

Indolestes alleni female
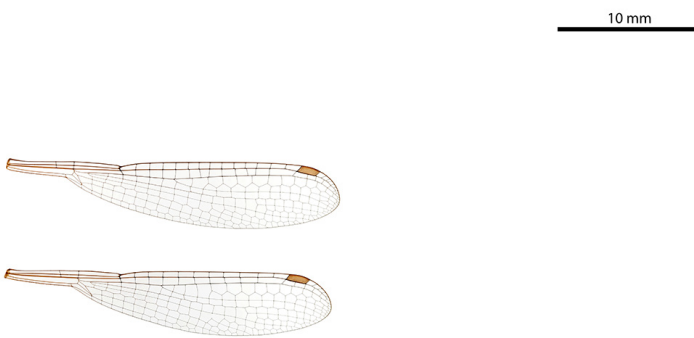

Indolestes obiri female
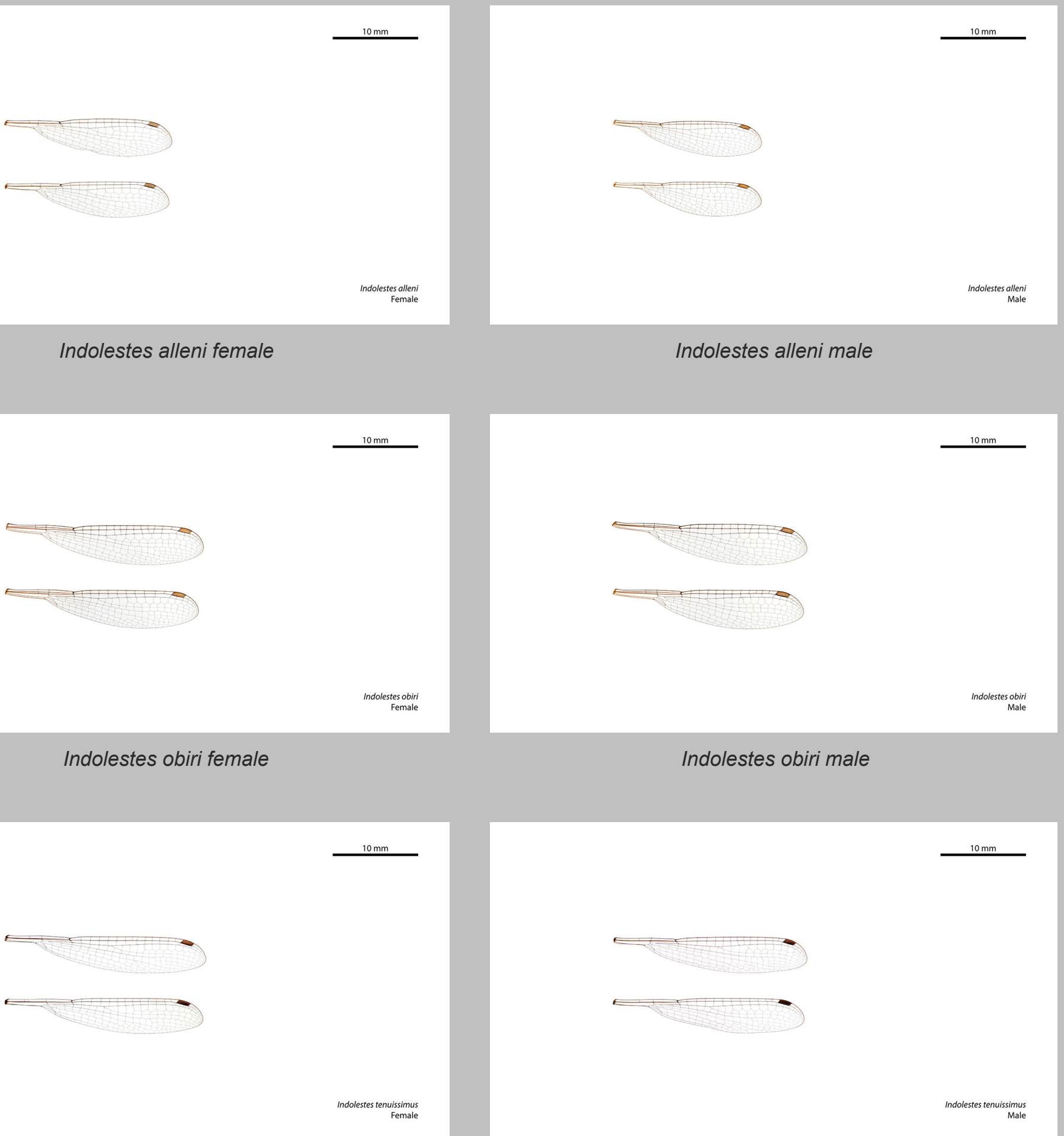

Indolestes alleni male

Hemiphlebia mirabilis male

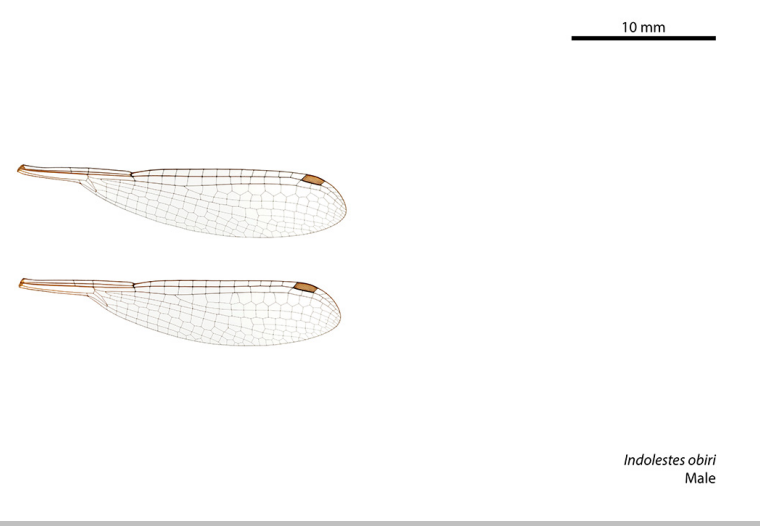

Indolestes obiri male

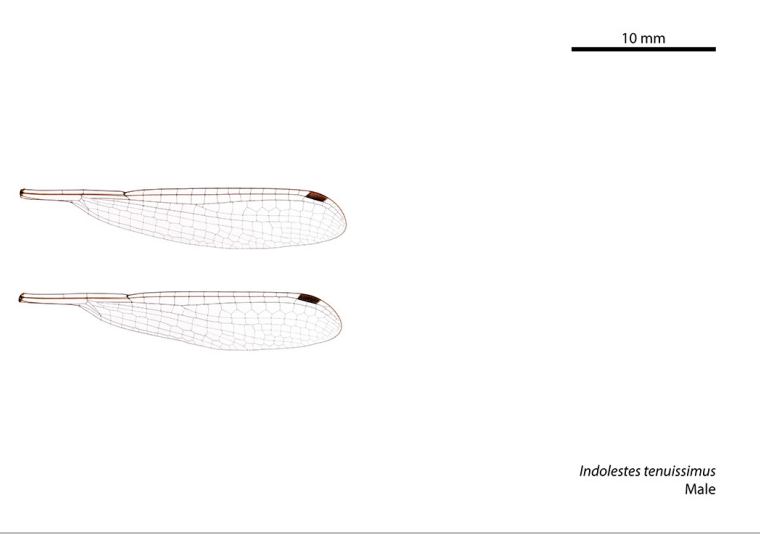

Indolestes tenuissimus male 


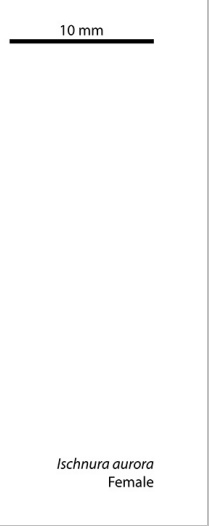

Ischnura aurora female

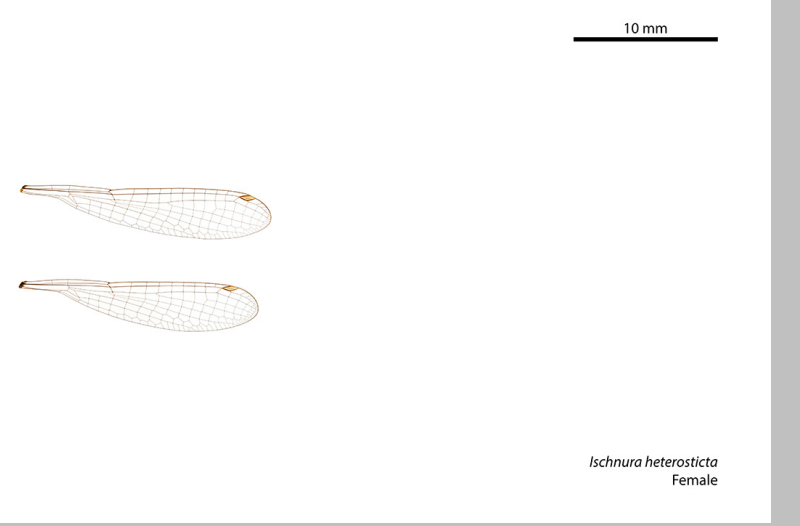

Ischnura heterosticta female

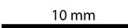

Ischnura pruinescens
Female

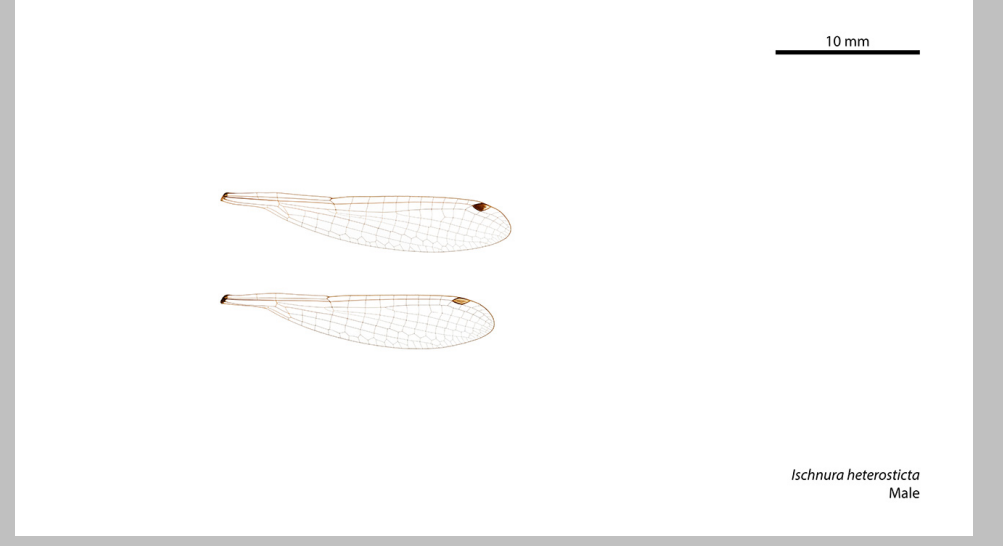

Ischnura heterosticta male

Ischnura aurora male

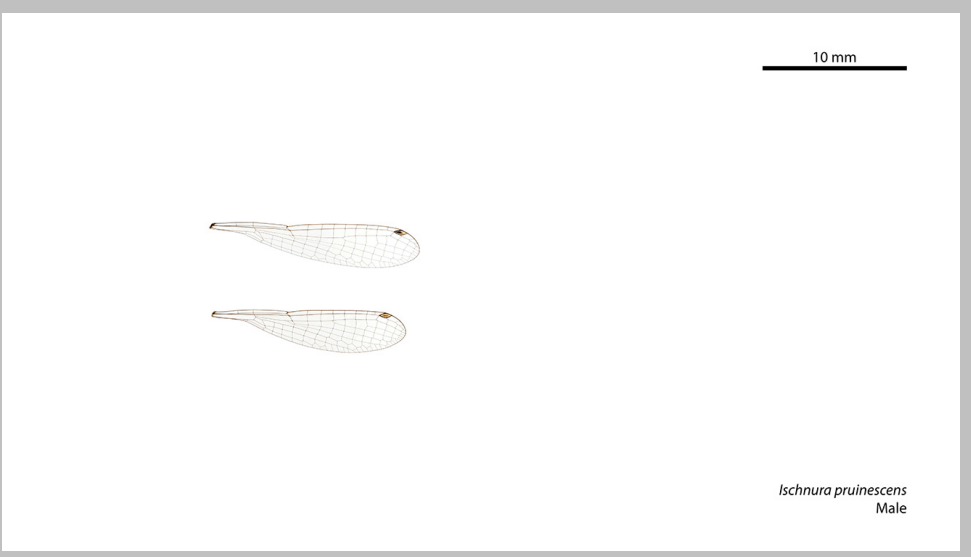

Ischnura pruinescens male
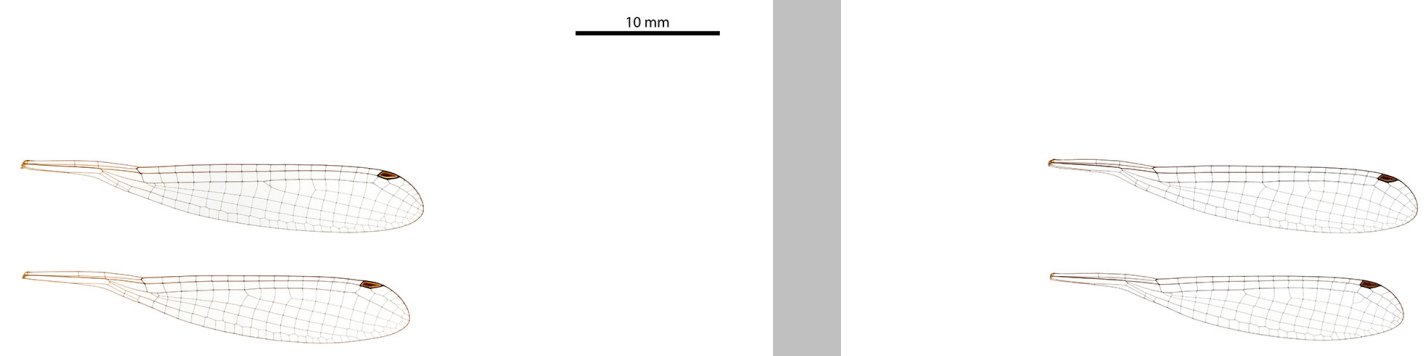


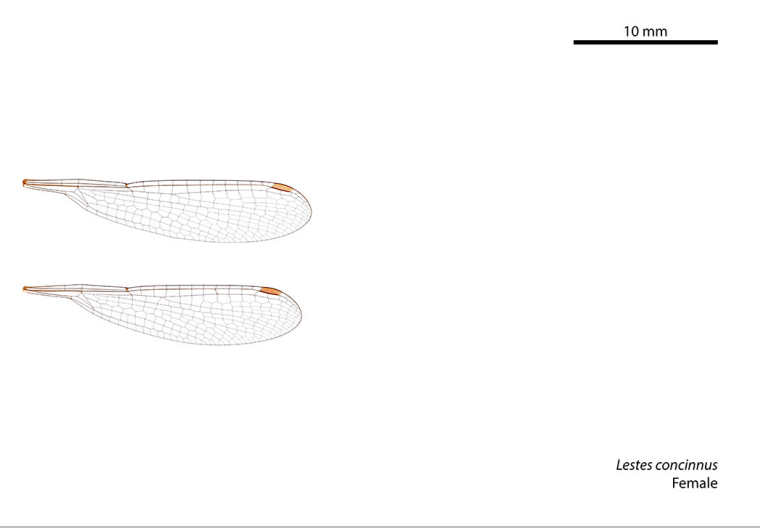

Lestes concinnus female

Lestoidea barbarae female

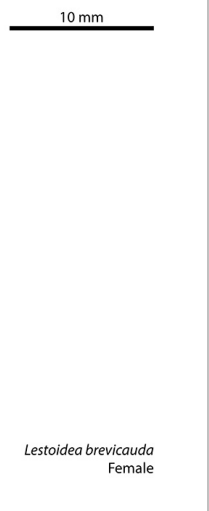

Lestoidea brevicauda female

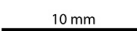

Lestoidea conjuncta

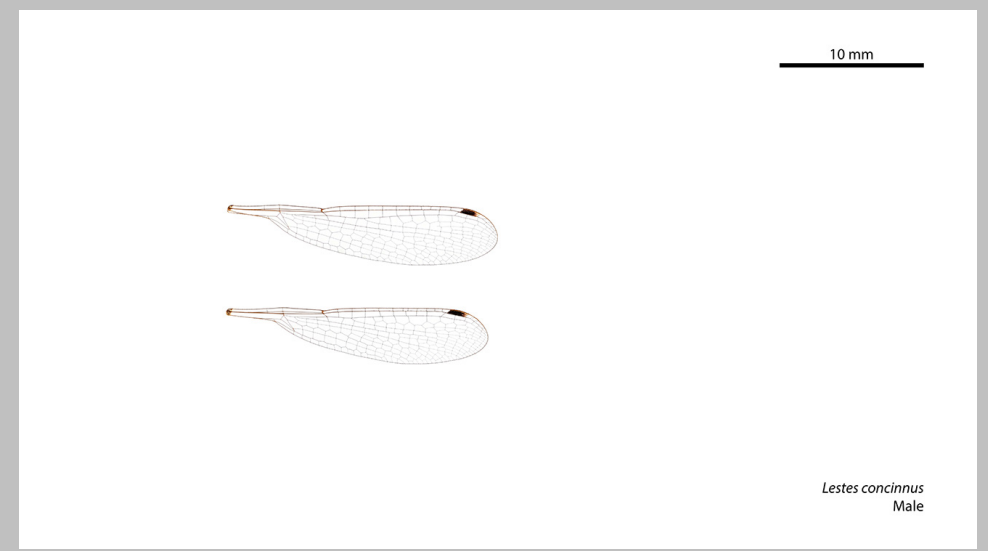

Lestes concinnus male

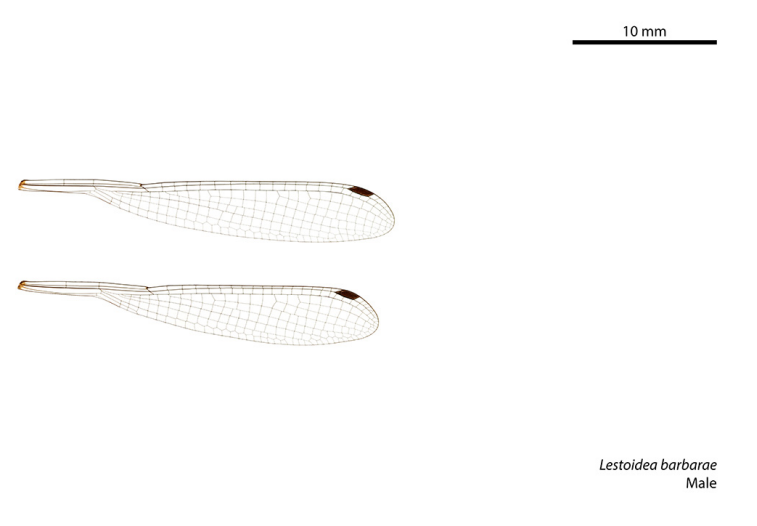

Lestoidea barbarae male

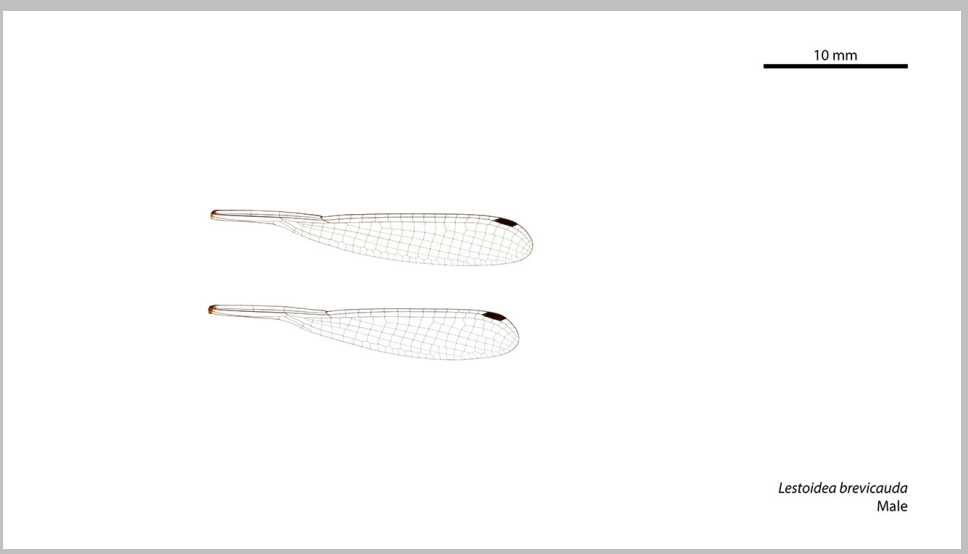

Lestoidea brevicauda male

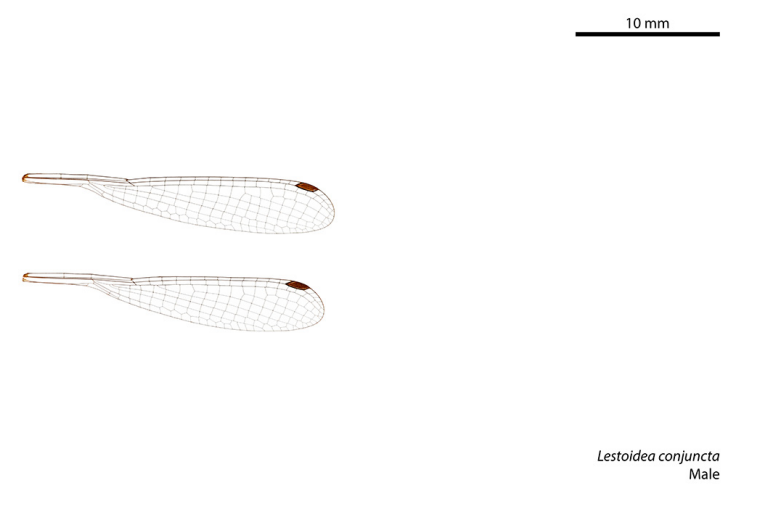

Lestoidea conjuncta male 


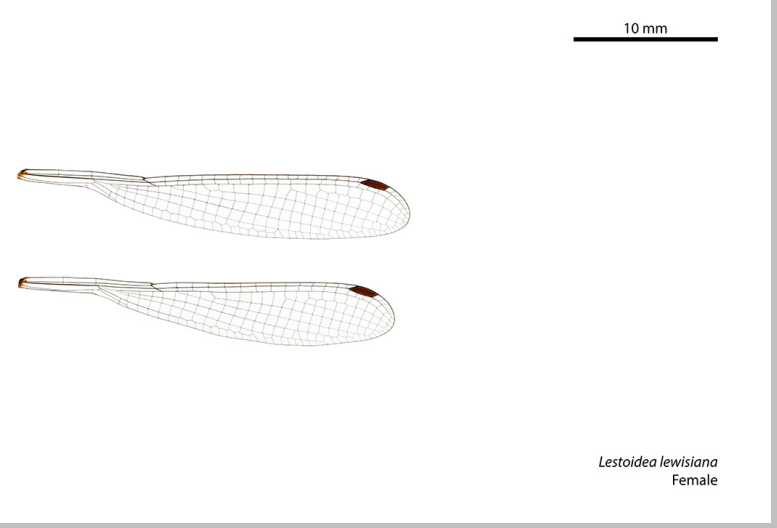

Lestoidea lewisiana female

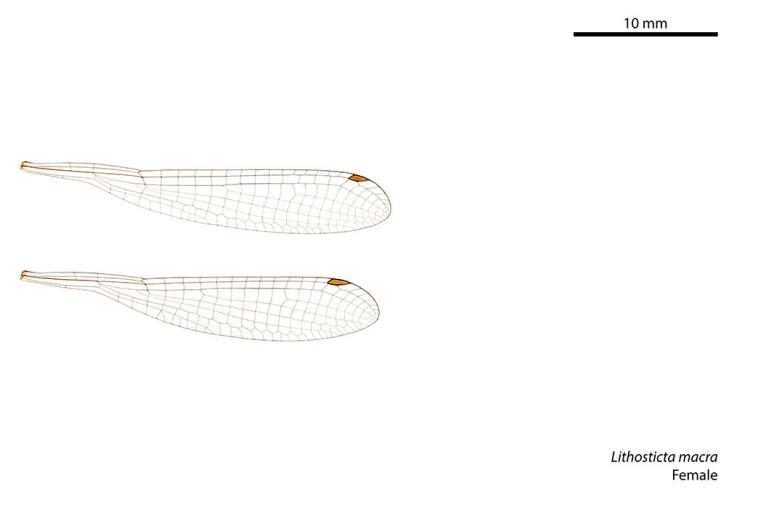

Lithosticta macra female

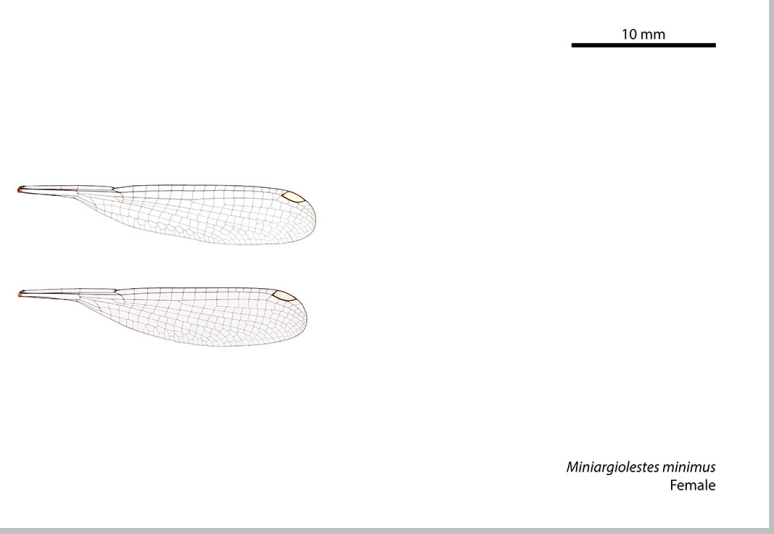

Miniargiolestes minimus female

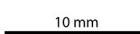

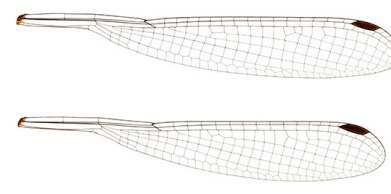

Lestoidea lewisiana male

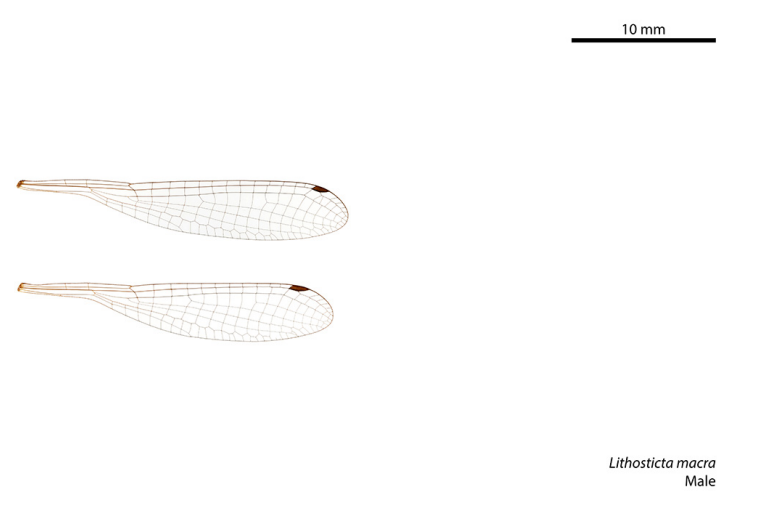

Lithosticta macra male

Miniargiolestes minimus
Male

Miniargiolestes minimus male

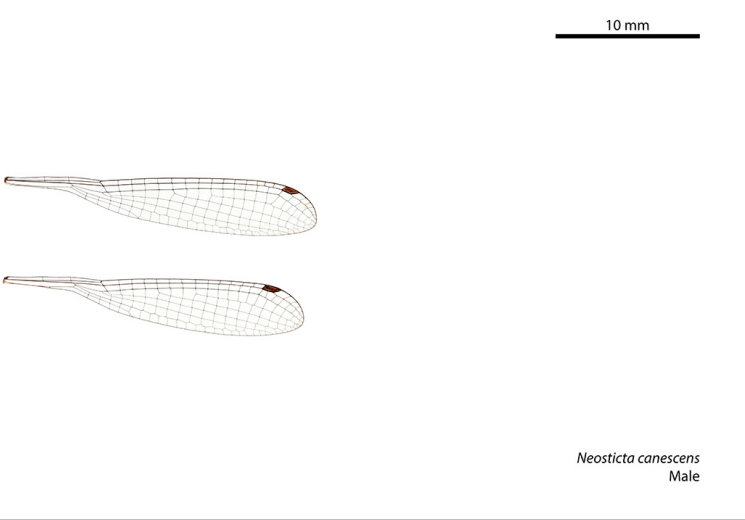

Neosticta canescens male 


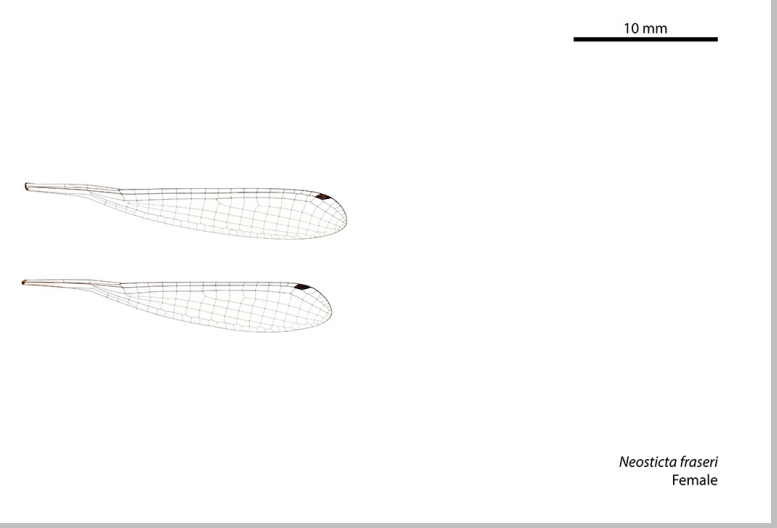

Neosticta fraseri female

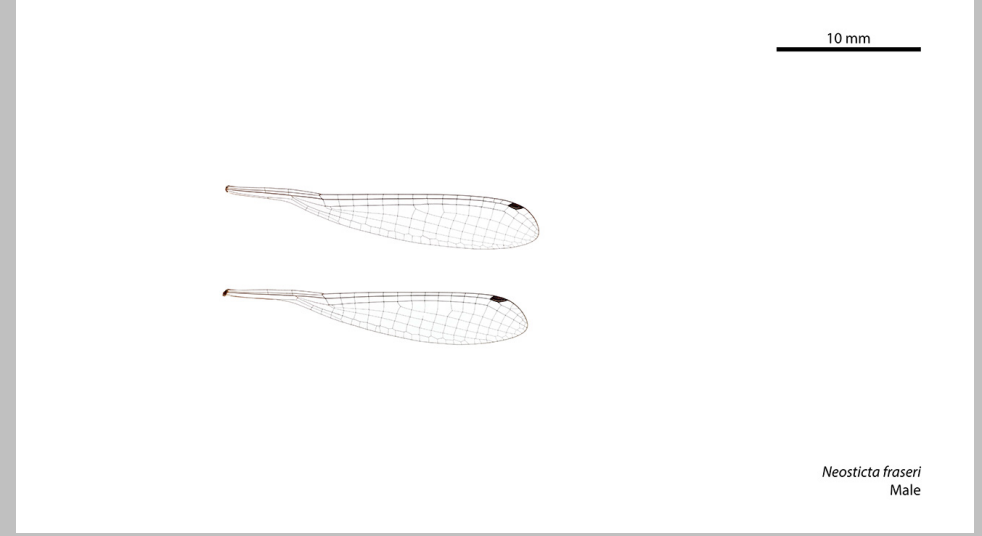

Neosticta fraseri male

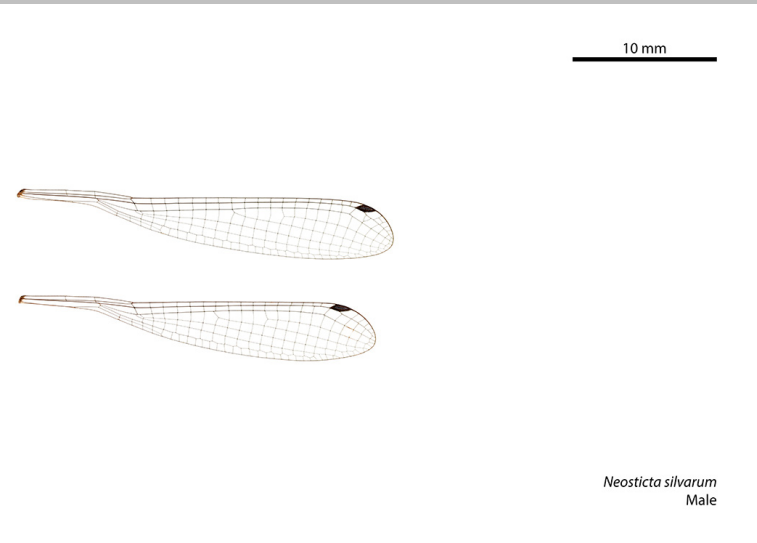

Neosticta silvarum male
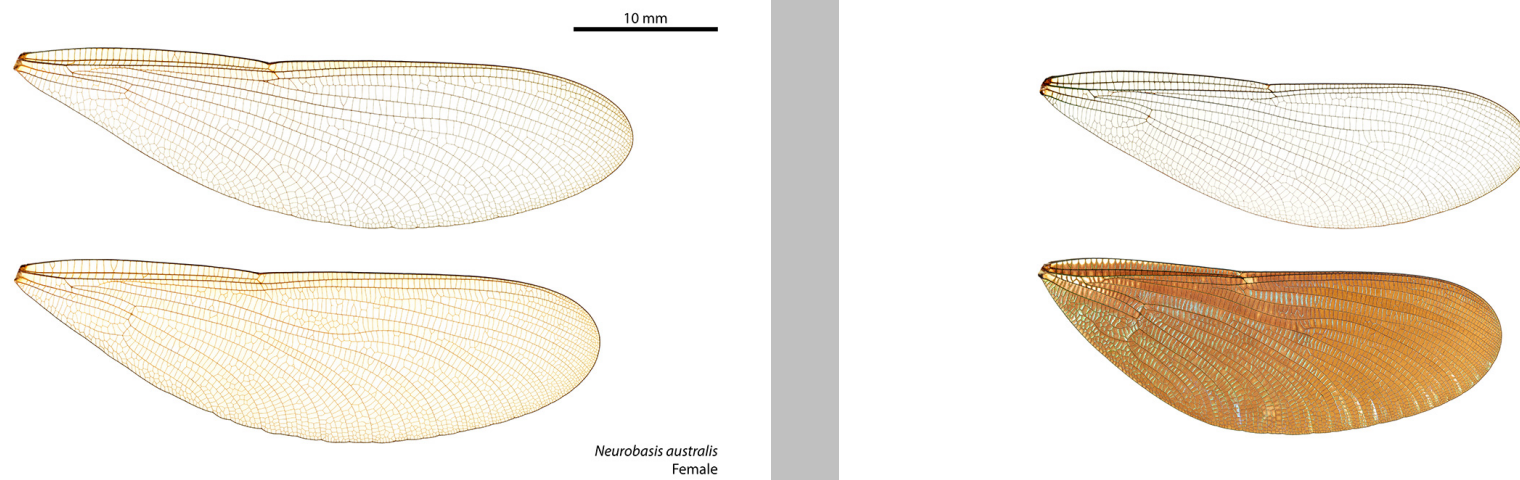

Neurobasis australis

Neurobasis australis female 


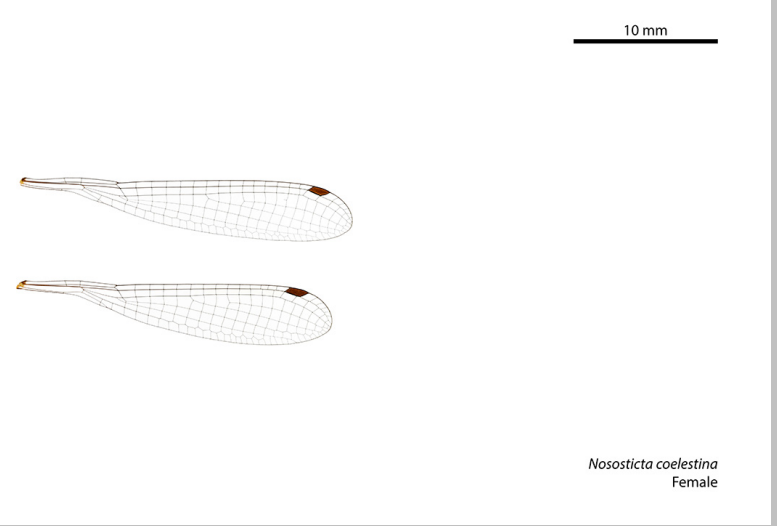

Nososticta coelestina female

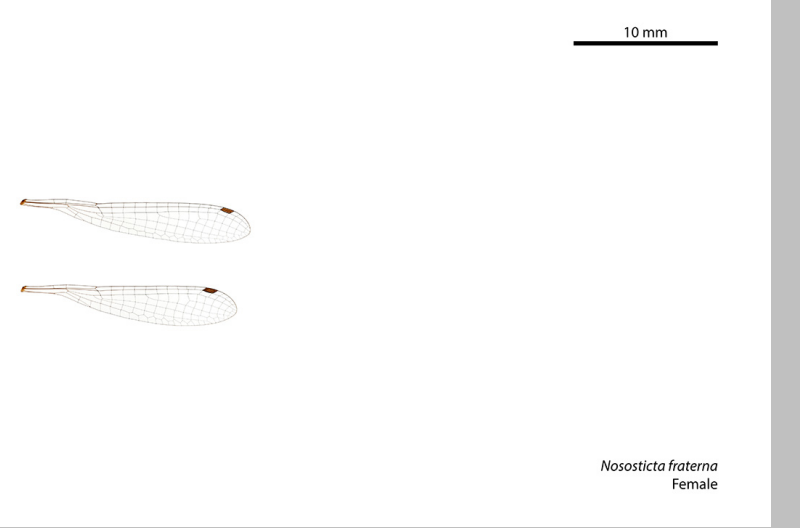

Nososticta fraterna female

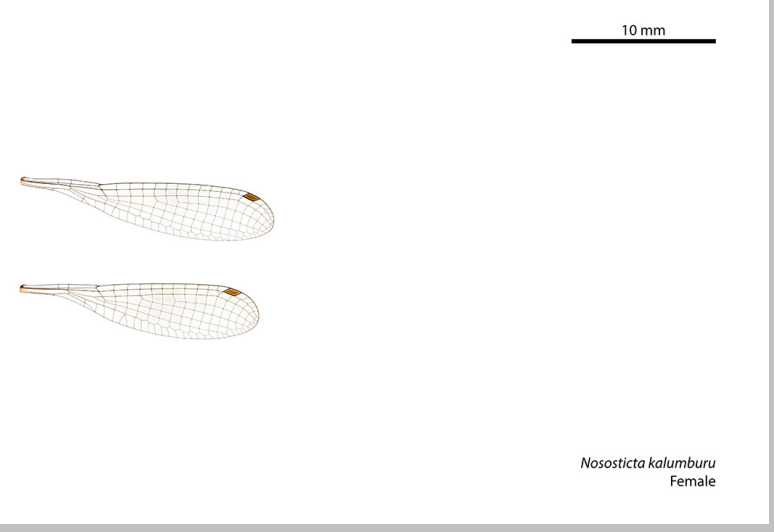

Nososticta kalumburu female

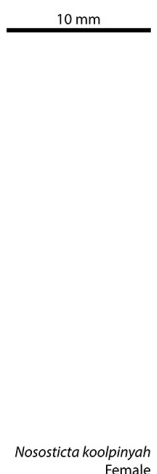

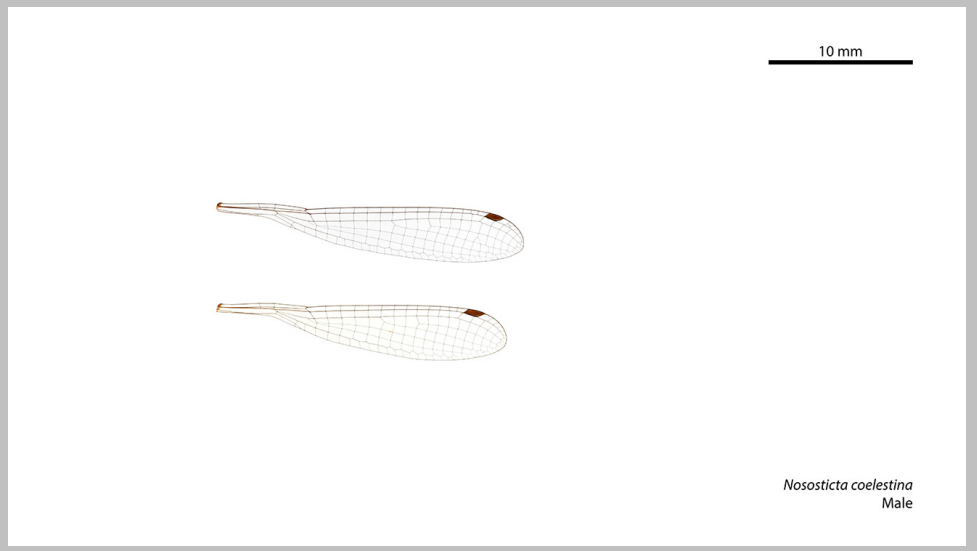

Nososticta coelestina male

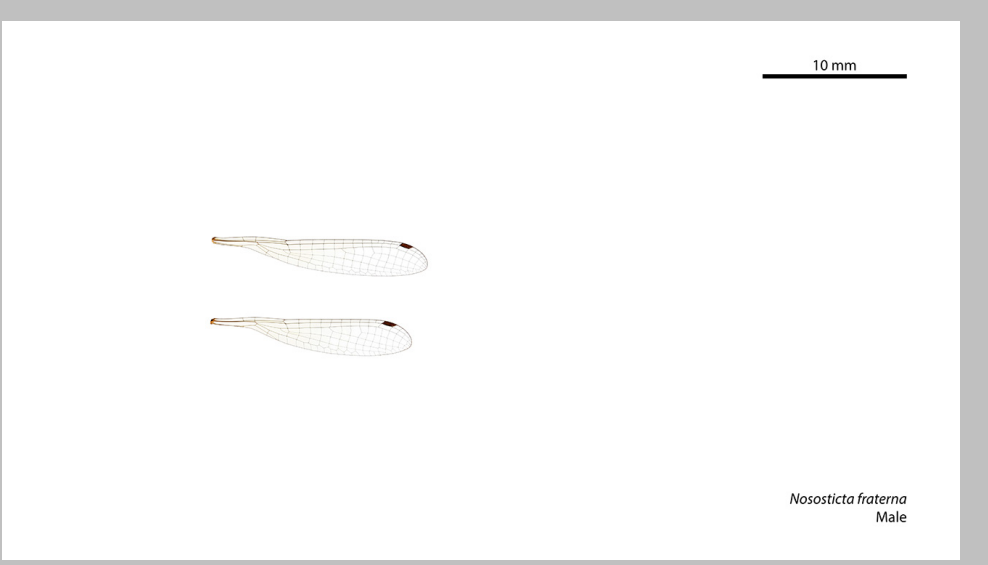

Nososticta fraterna male

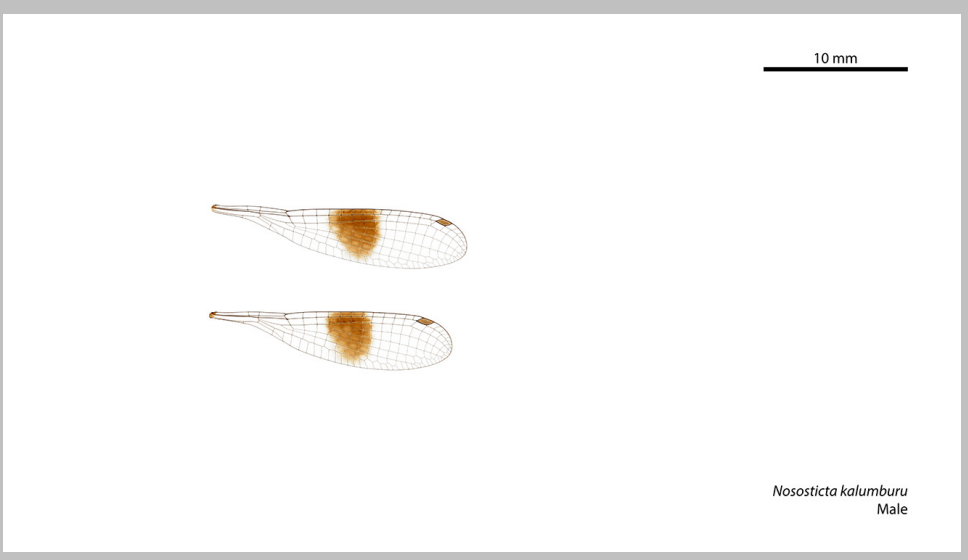

Nososticta kalumburu male

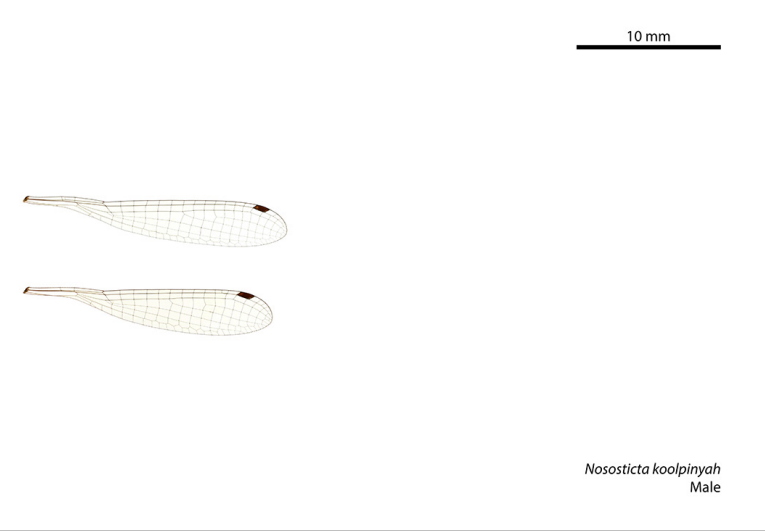

Nososticta koolpinyah male 


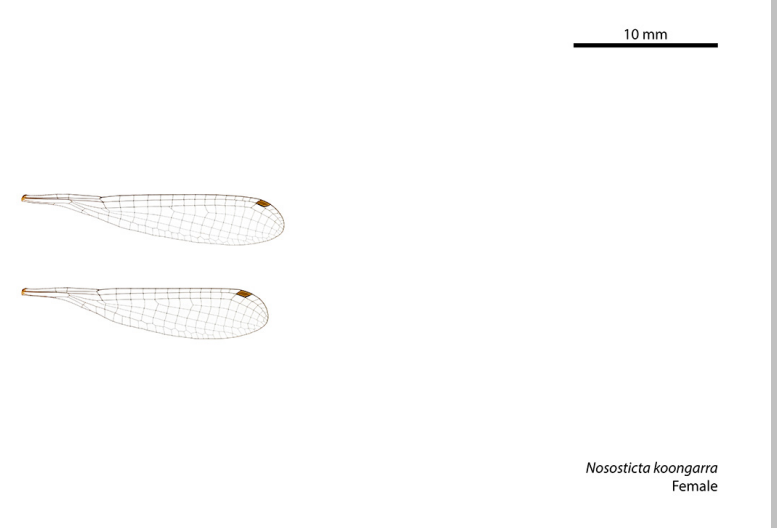

Nososticta koongarra female

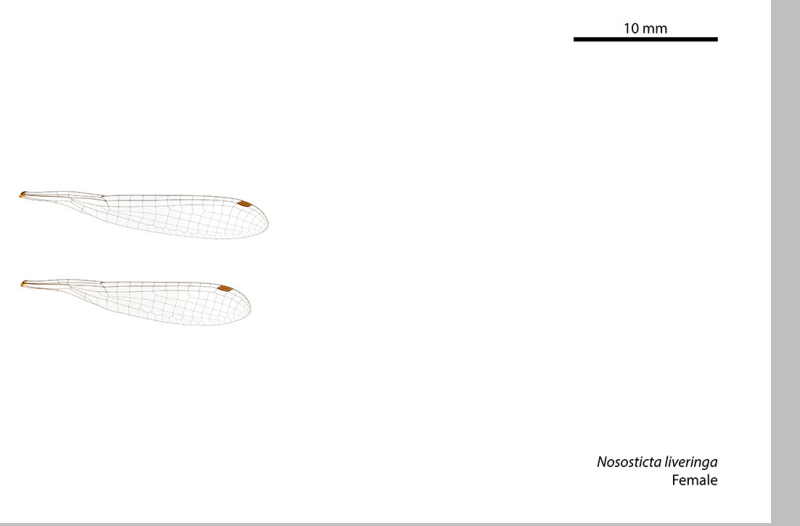

Nososticta liveringa female

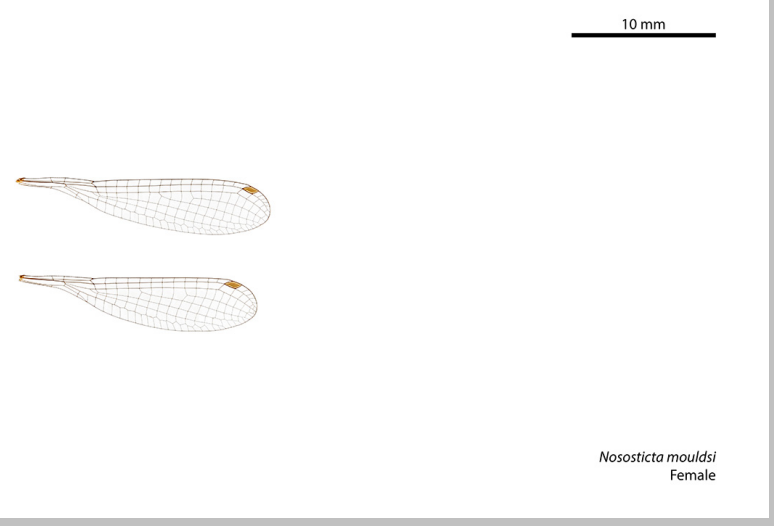

Nososticta mouldsi female

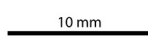

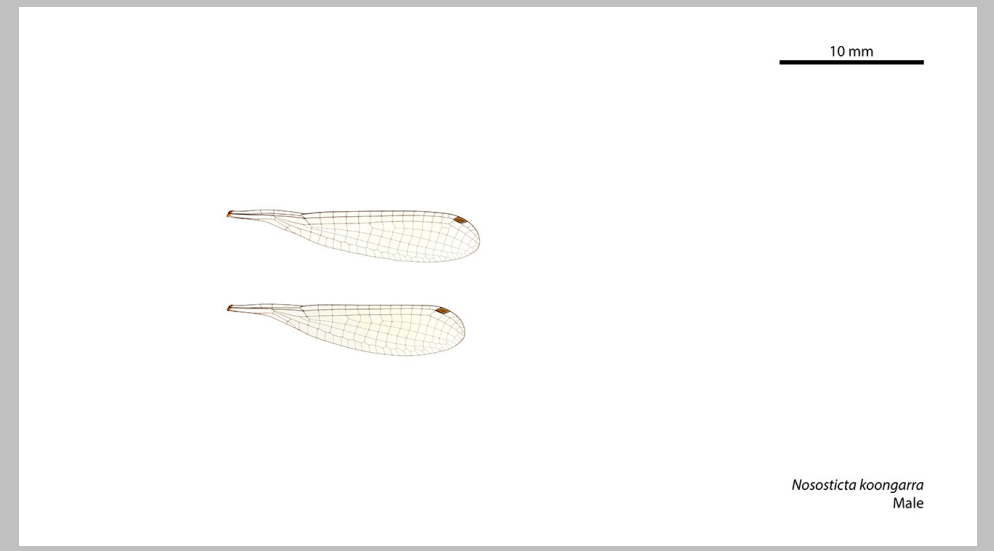

Nososticta koongarra male

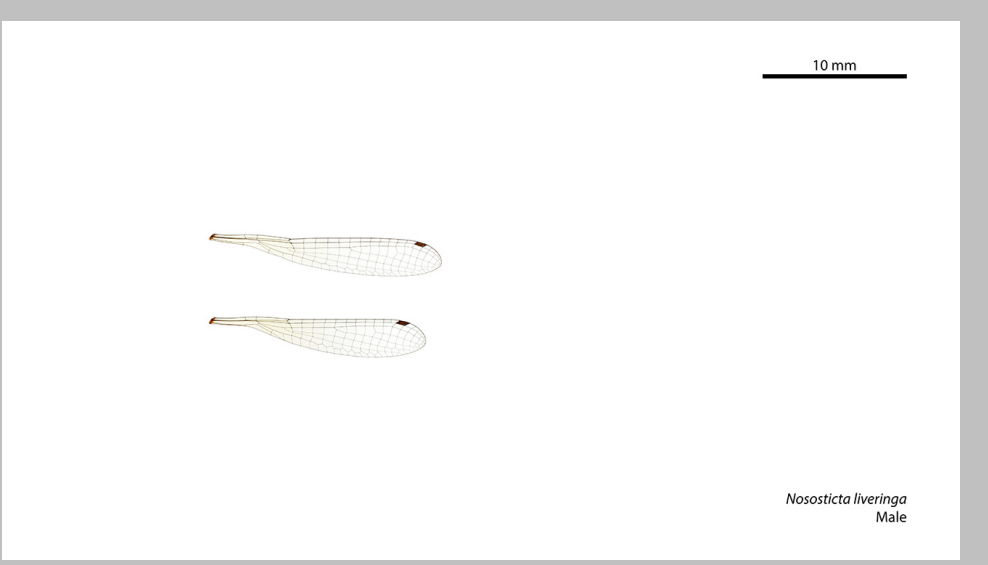

Nososticta liveringa male

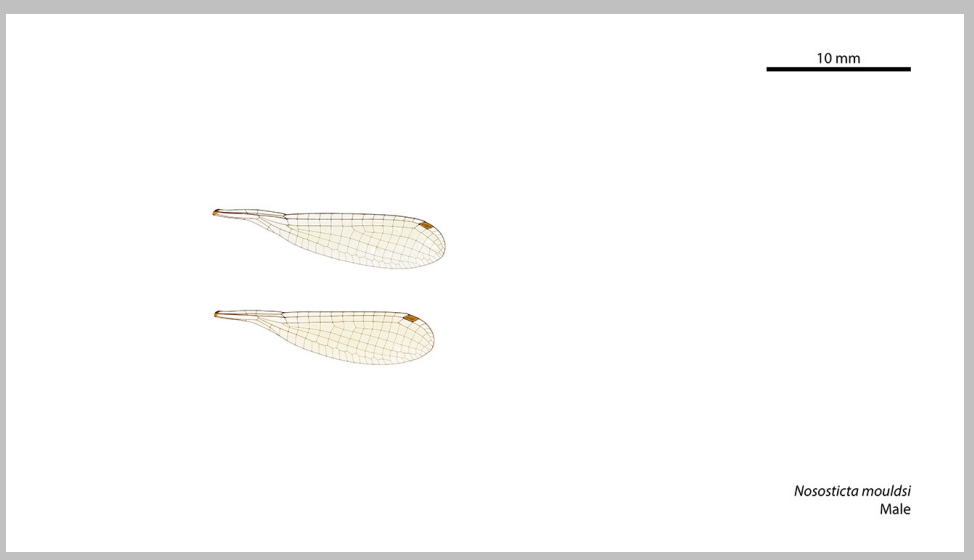

Nososticta mouldsi male 


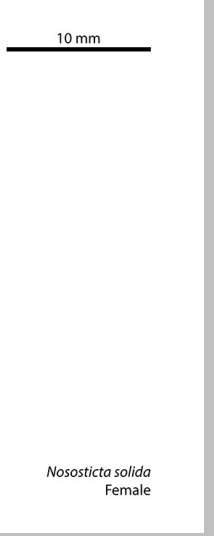

Nososticta solida female

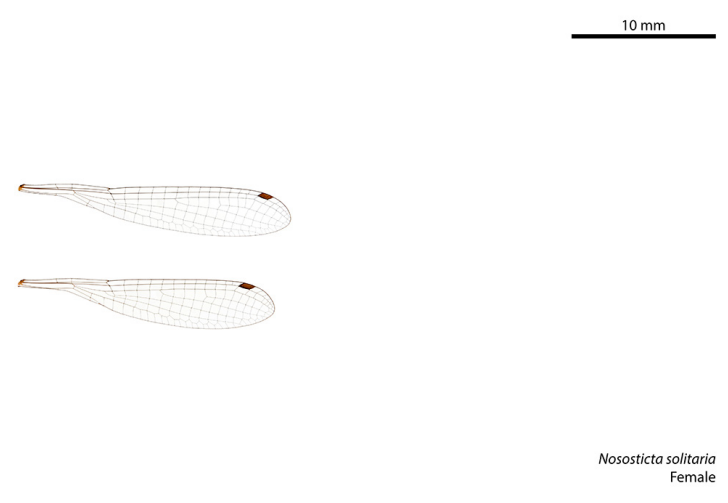

Nososticta solitaria female

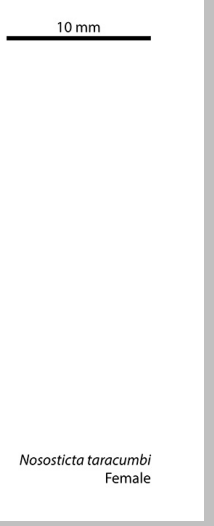

Nososticta taracumbi female

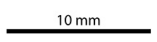

Oristicta filicicola
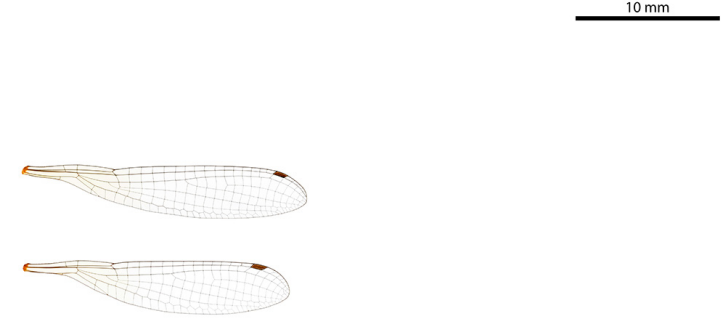

Nososticta solida male

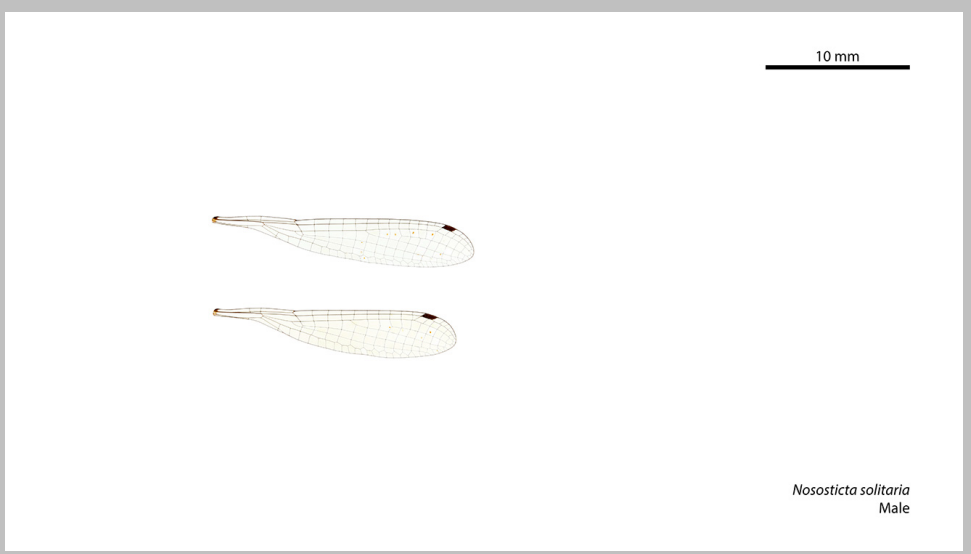

Nososticta solitaria male

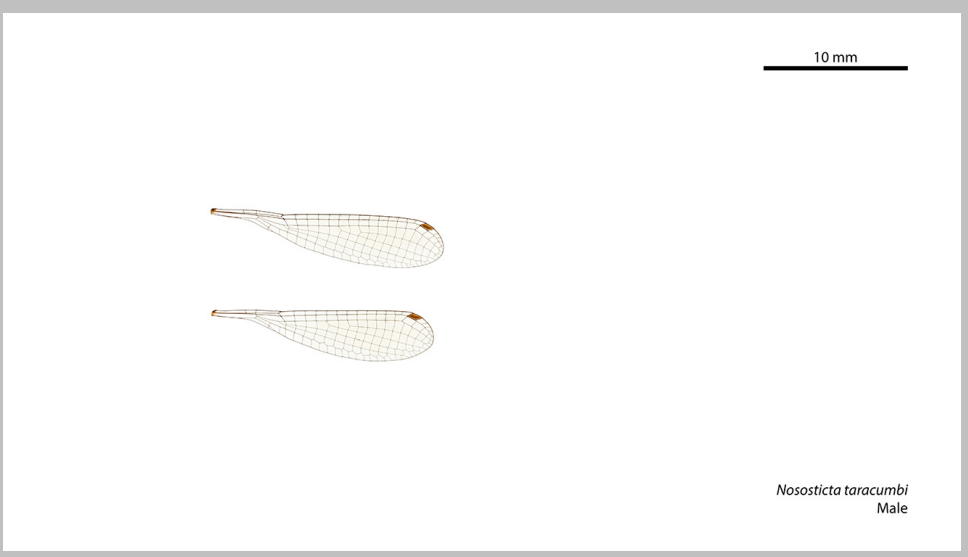

Nososticta taracumbi male 


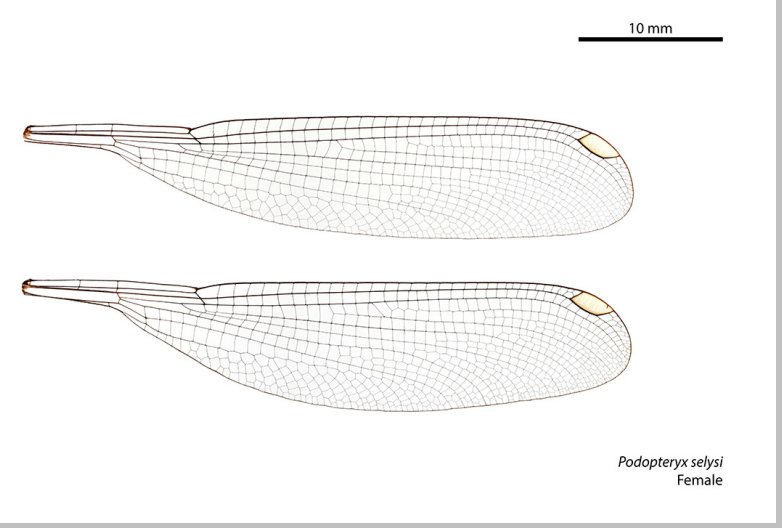

Podopteryx selysi female

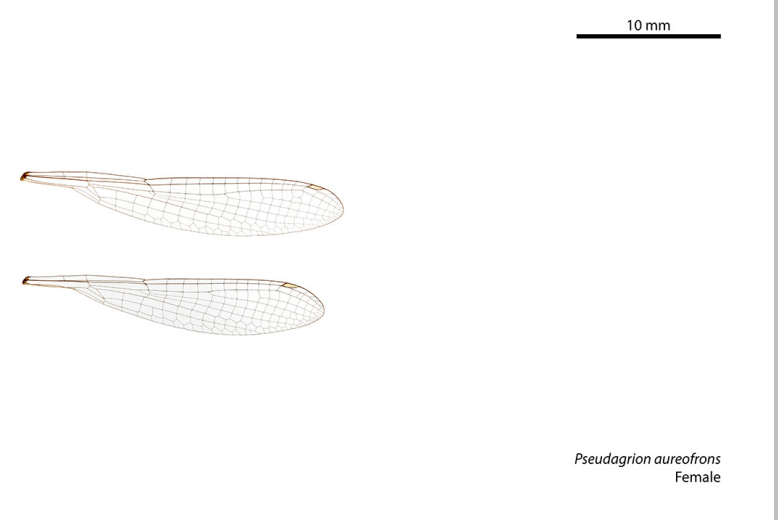

Pseudagrion aureofrons female

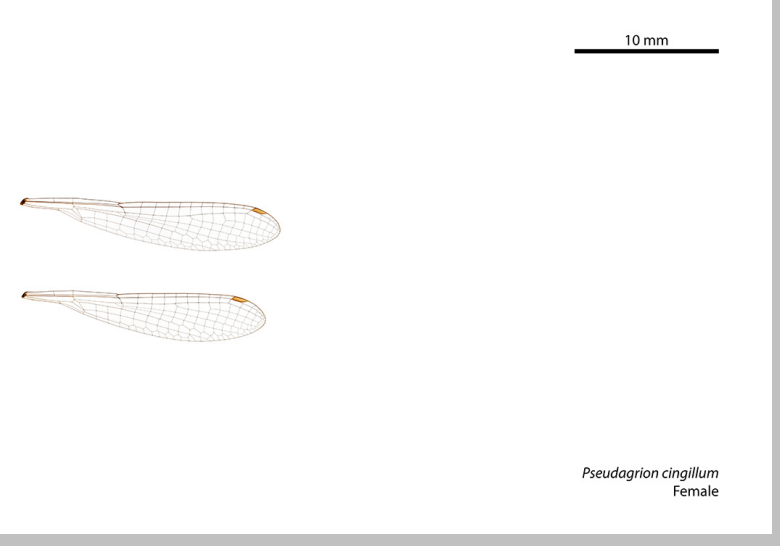

Pseudagrion cingillum female
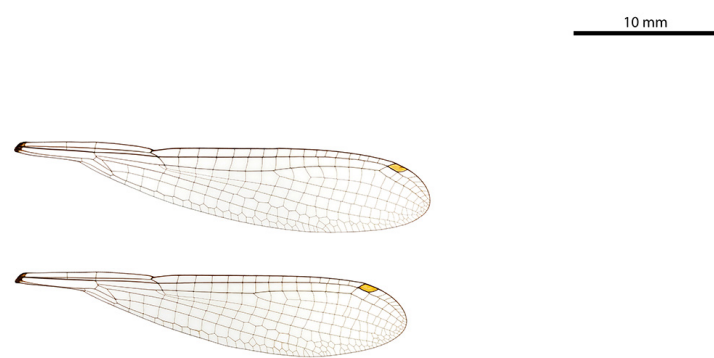

Podopteryx selysi male

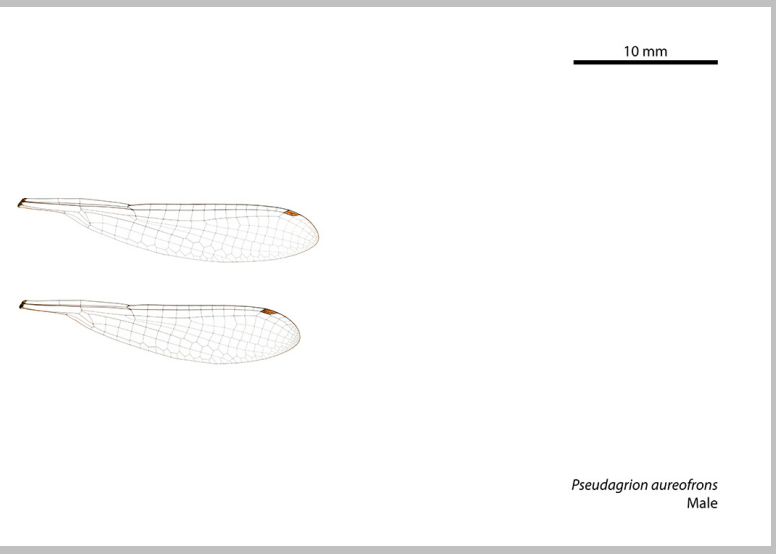

Pseudagrion aureofrons male

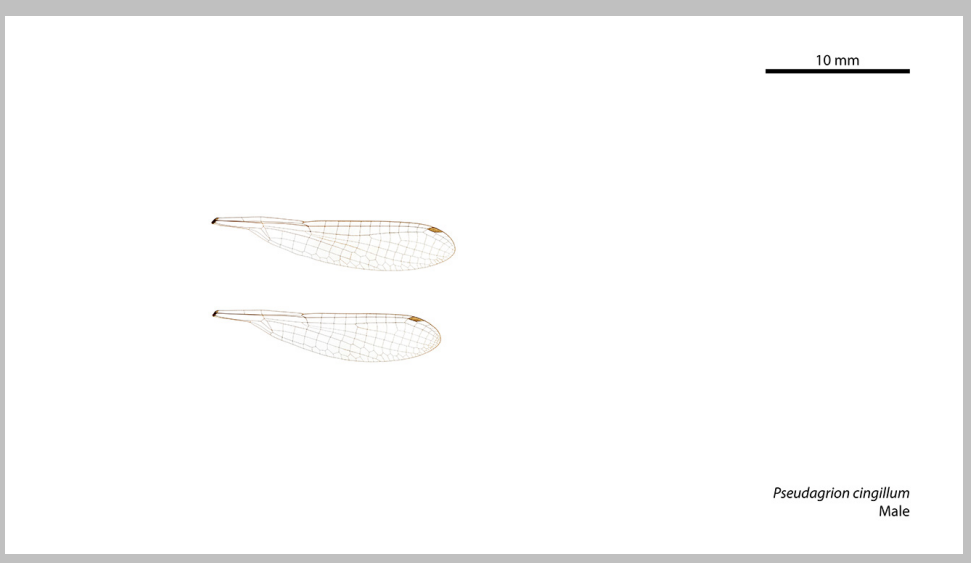

Pseudagrion cingillum male

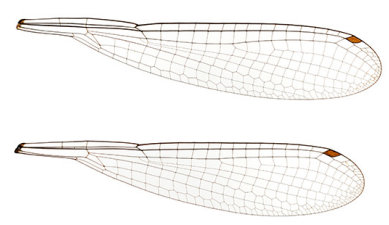




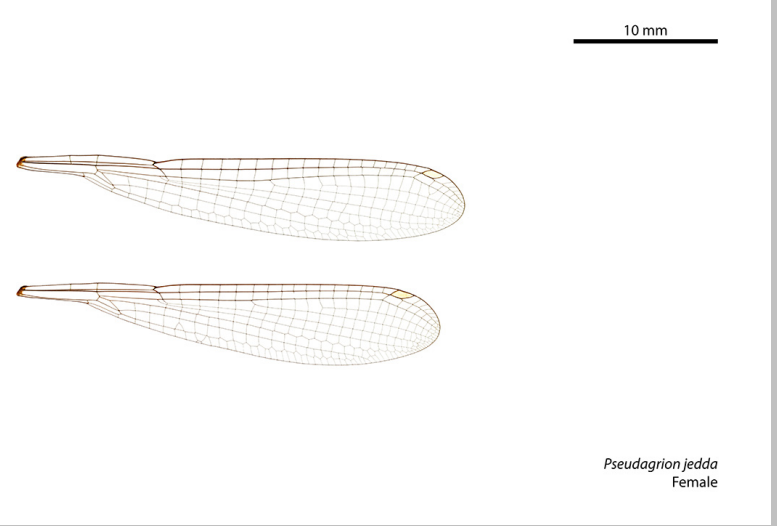

Pseudagrion jedda female

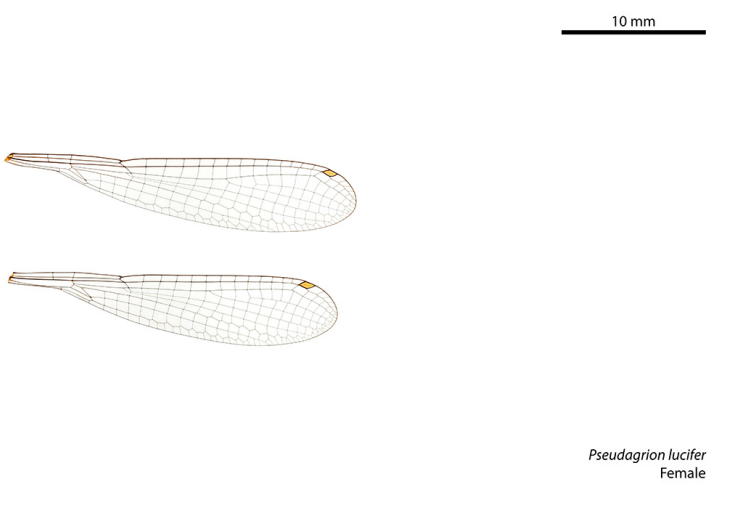

Pseudagrion lucifer female

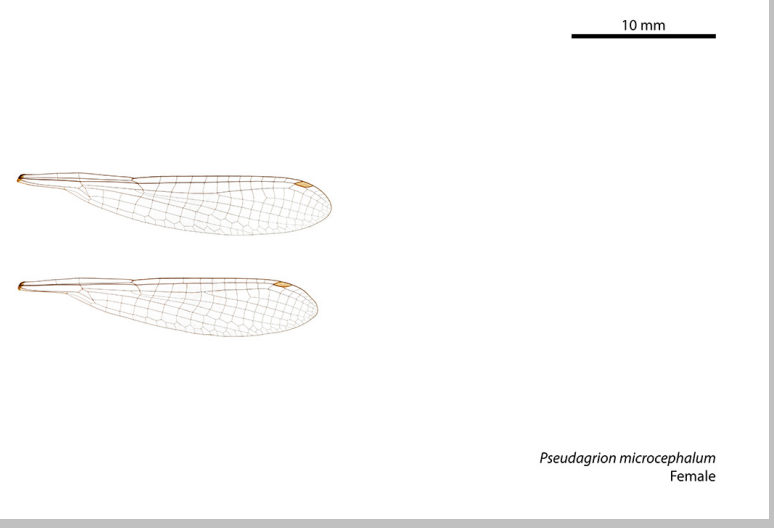

Pseudagrion microcephalum female

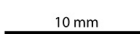

Rhadinosticta banksi
Female

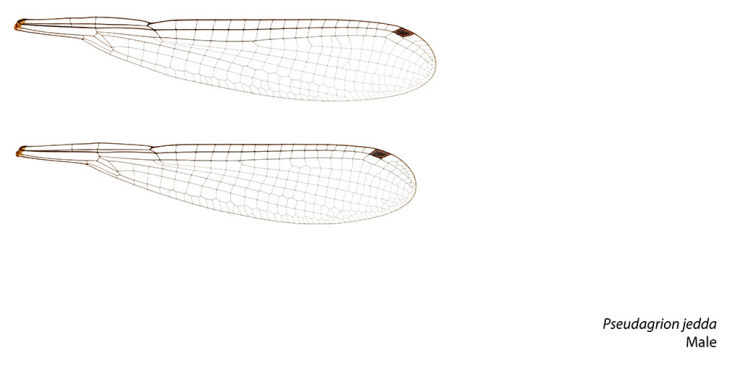

Pseudagrion jedda male

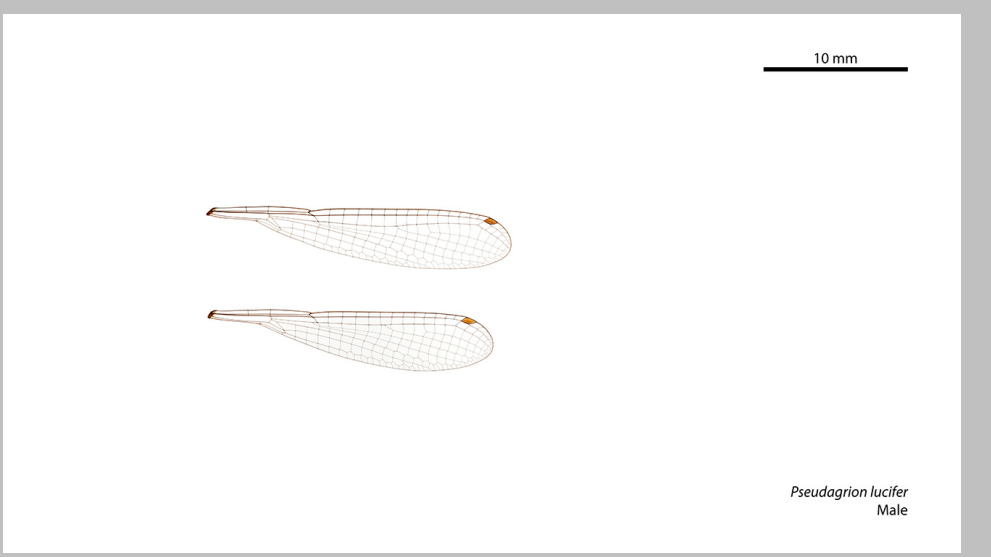

Pseudagrion lucifer male

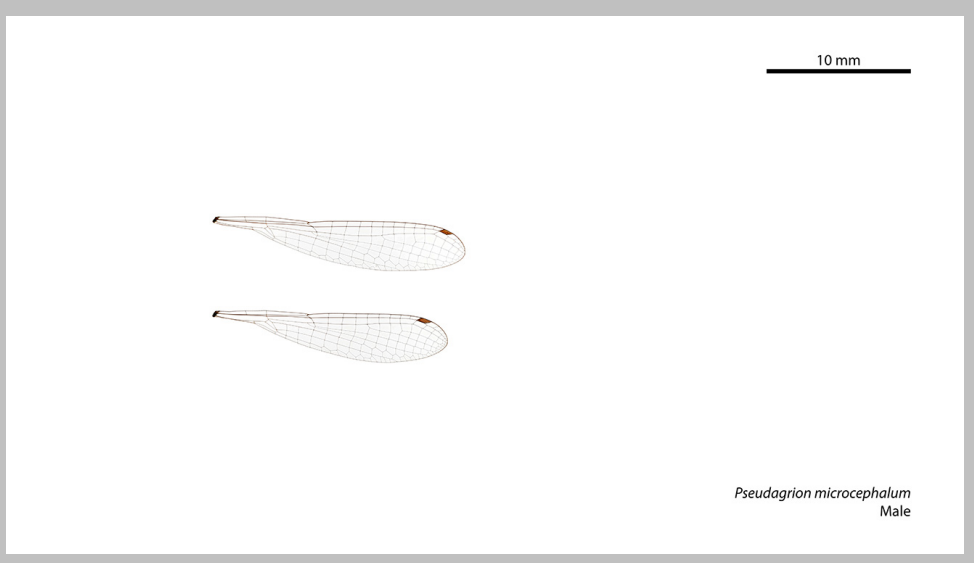

Pseudagrion microcephalum male

Rhadinosticta banks 


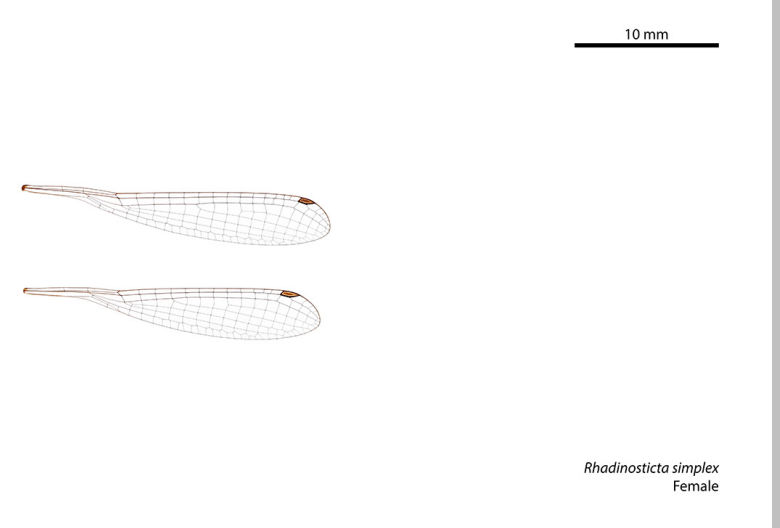

Rhadinosticta simplex female

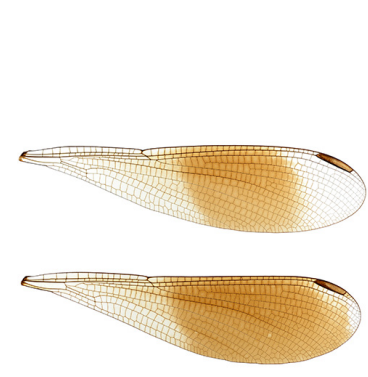

Rhinocypha tincta
Female

Rhinocypha tincta female
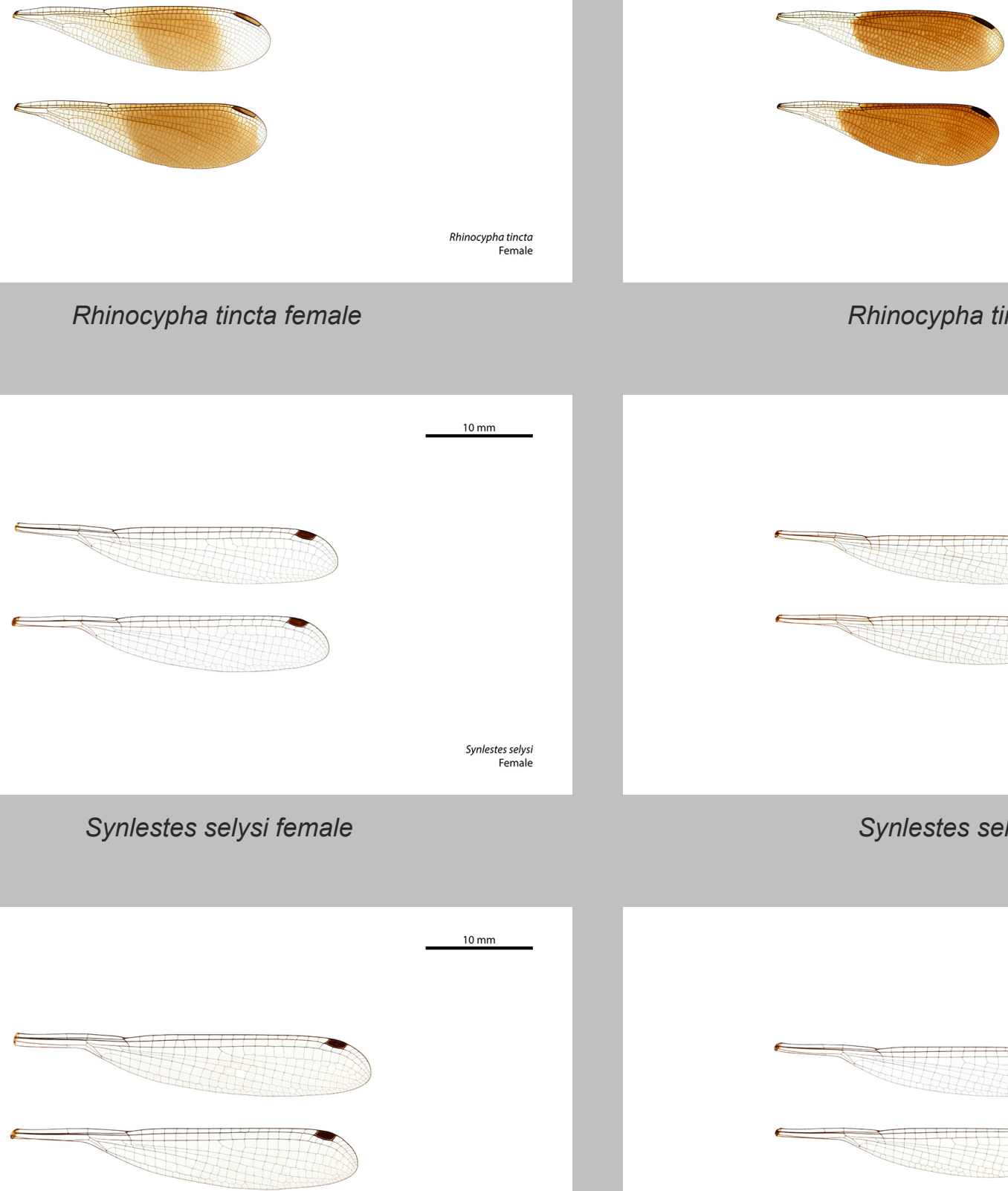

Rhinocypha tincta male

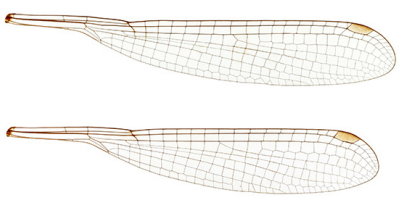

Synlestes selysi male

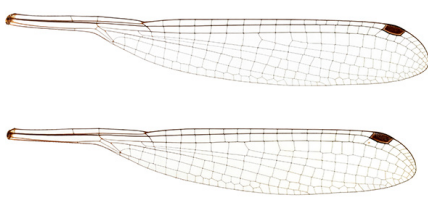




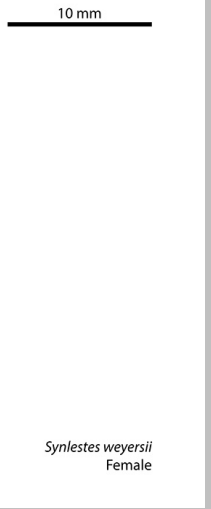

Synlestes weyersii female

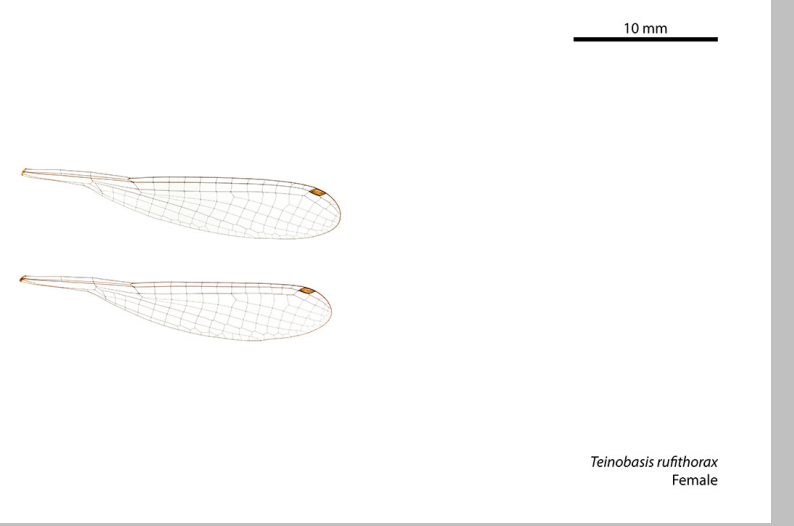

Teinobasis rufithorax female

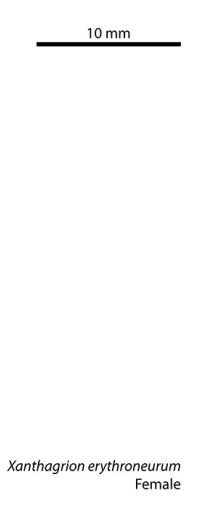

Xanthagrion erythroneurum female

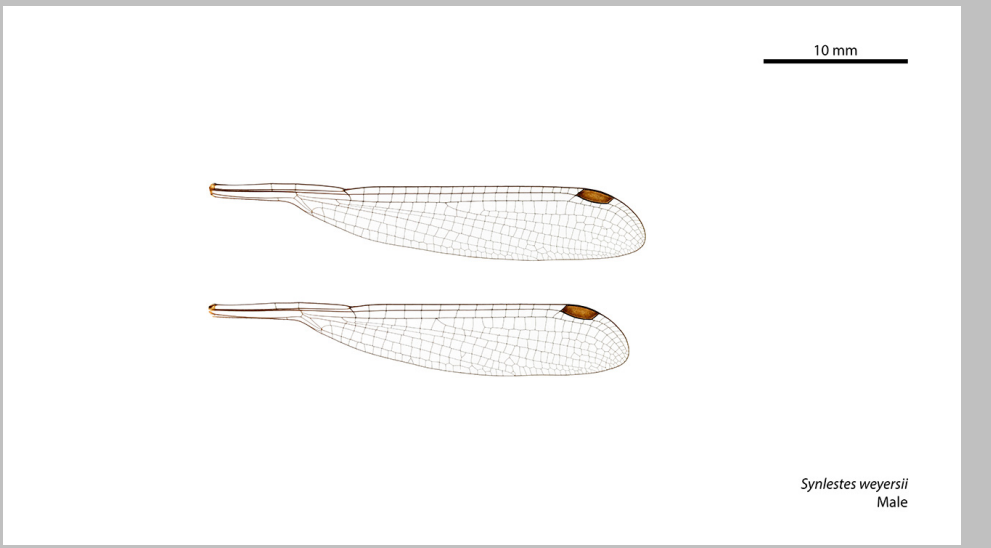

Synlestes weyersii male

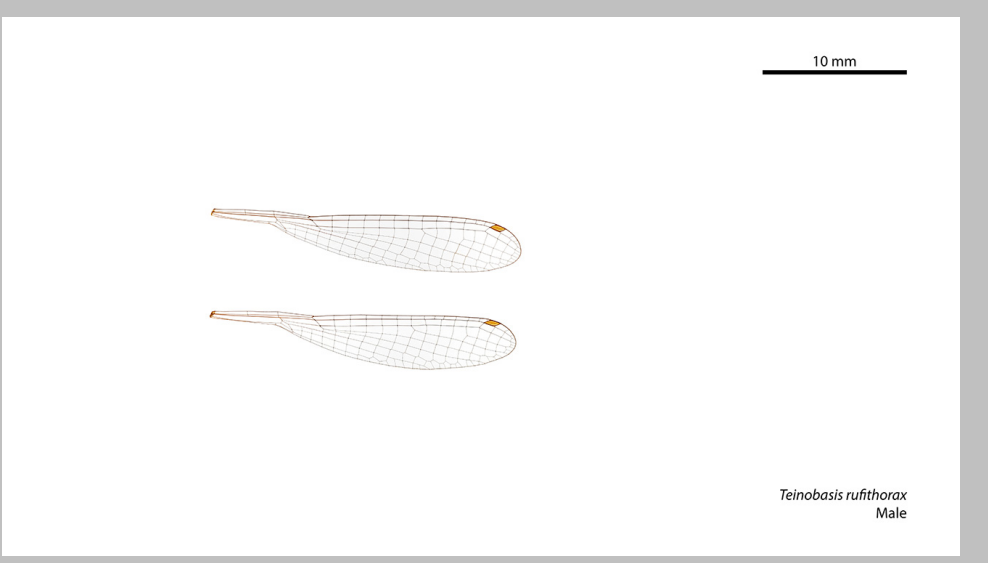

Teinobasis rufithorax male

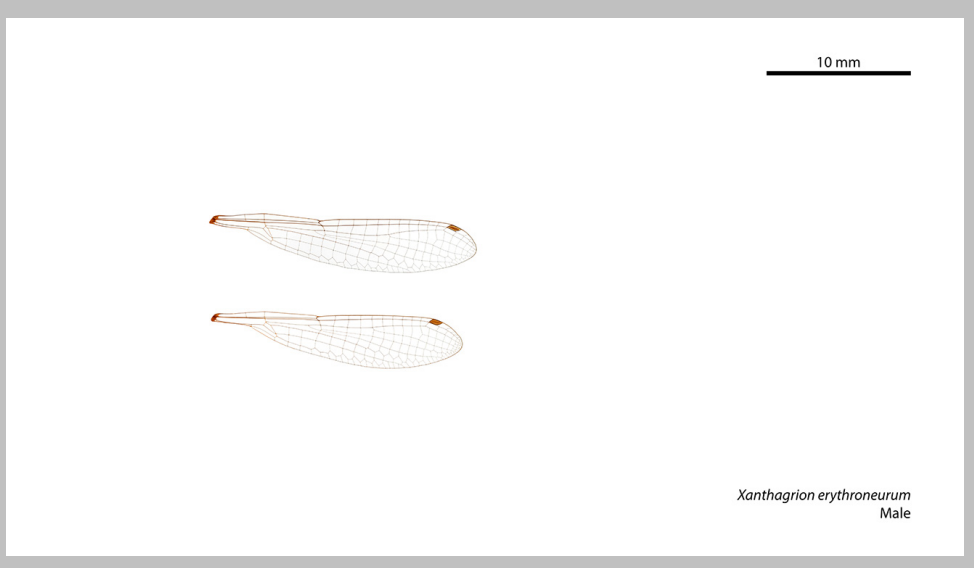

Xanthagrion erythroneurum male 


\section{Appendix 3-Index to images}

Index to full-size images. This table is an abridged version of the full Index published at figshare (Tann, 2020e). Species are arranged alphabetically. For common names, hyperlinks to the Atlas of Living Australia (ALA) specimens records, additional metadata, and specimen images, see Tann (2020e). Abbreviations: Coll., museum or institutional collection; Coll.Reg., registration number; AM, Australian Museum, Sydney; ANIC, Australian National Insect Collection, Canberra; MAGNT, Museum and Art Gallery of the Northern Territory, Darwin; $Q M$, Queensland Museum, Brisbane; and WAM, Western Australian Museum, Perth.

\begin{tabular}{|c|c|c|c|c|c|}
\hline family & species & sex & Coll. & Coll.Reg. & figshare DOI for wing images \\
\hline Telephlebiidae & Acanthaeschna victoria & $\mathrm{F}$ & ANIC & $7-009684$ & https://doi.org/10.6084/m9.figshare.12605939 \\
\hline Telephlebiidae & Acanthaeschna victoria & M & $\mathrm{AM}$ & K300814 & https://doi.org/10.6084/m9.figshare.12605939 \\
\hline Coenagrionidae & Aciagrion fragilis & $\mathrm{F}$ & $\mathrm{AM}$ & K301206 & https://doi.org/10.6084/m9.figshare.12597695 \\
\hline Coenagrionidae & Aciagrion fragilis & M & $\mathrm{AM}$ & K301207 & https://doi.org/10.6084/m9.figshare.12597695 \\
\hline Aeshnidae & Adversaeschna brevistyla & $\mathrm{F}$ & $\mathrm{AM}$ & K305467 & https://doi.org/10.6084/m9.figshare. 12605936 \\
\hline Aeshnidae & Adversaeschna brevistyla & M & $\mathrm{AM}$ & K305480 & https://doi.org/10.6084/m9.figshare. 12605936 \\
\hline Libellulidae & Aethriamanta circumsignata & $\mathrm{F}$ & ANIC & $7-011025$ & https://doi.org/10.6084/m9.figshare. 12605933 \\
\hline Libellulidae & Aethriamanta circumsignata & M & $\mathrm{AM}$ & K305539 & https://doi.org/10.6084/m9.figshare. 12605933 \\
\hline Libellulidae & Aethriamanta nymphaeae & $\mathrm{F}$ & ANIC & $7-011030$ & https://doi.org/10.6084/m9.figshare. 12605930 \\
\hline Libellulidae & Aethriamanta nymphaeae & $\mathrm{M}$ & ANIC & $7-011033$ & https://doi.org/10.6084/m9.figshare. 12605930 \\
\hline Coenagrionidae & Agriocnemis argentea & $\mathrm{F}$ & $\mathrm{AM}$ & K305648 & https://doi.org/10.6084/m9.figshare.12597692 \\
\hline Coenagrionidae & Agriocnemis argentea & M & $\mathrm{AM}$ & K301205 & https://doi.org/10.6084/m9.figshare. 12597692 \\
\hline Coenagrionidae & Agriocnemis dobsoni & $\mathrm{F}$ & ANIC & $7-001968$ & https://doi.org/10.6084/m9.figshare.12597683 \\
\hline Coenagrionidae & Agriocnemis dobsoni & M & ANIC & $7-001969$ & https://doi.org/10.6084/m9.figshare. 12597683 \\
\hline Coenagrionidae & Agriocnemis femina & $\mathrm{F}$ & ANIC & $7-001971$ & https://doi.org/10.6084/m9.figshare. 12597677 \\
\hline Coenagrionidae & Agriocnemis femina & M & ANIC & $7-001971$ & https://doi.org/10.6084/m9.figshare.12597677 \\
\hline Coenagrionidae & Agriocnemis kunjina & $\mathrm{F}$ & $\mathrm{AM}$ & K280879 & https://doi.org/10.6084/m9.figshare. 12597671 \\
\hline Coenagrionidae & Agriocnemis kunjina & M & $\mathrm{AM}$ & K305666 & https://doi.org/10.6084/m9.figshare.12597671 \\
\hline Coenagrionidae & Agriocnemis pygmaea & $\mathrm{F}$ & $\mathrm{AM}$ & K301197 & https://doi.org/10.6084/m9.figshare. 12597668 \\
\hline Coenagrionidae & Agriocnemis pygmaea & M & $\mathrm{AM}$ & K337755 & https://doi.org/10.6084/m9.figshare. 12597668 \\
\hline Coenagrionidae & Agriocnemis rubricauda & $\mathrm{F}$ & $\mathrm{AM}$ & K305665 & https://doi.org/10.6084/m9.figshare. 12597662 \\
\hline Coenagrionidae & Agriocnemis rubricauda & M & $\mathrm{AM}$ & K301187 & https://doi.org/10.6084/m9.figshare. 12597662 \\
\hline Libellulidae & Agrionoptera insignis & $\mathrm{F}$ & $\mathrm{AM}$ & K305525 & https://doi.org/10.6084/m9.figshare.12605921 \\
\hline Libellulidae & Agrionoptera insignis & M & $\mathrm{AM}$ & K337650 & https://doi.org/10.6084/m9.figshare.12605921 \\
\hline Libellulidae & Agrionoptera longitudinalis & $\mathrm{F}$ & $\mathrm{AM}$ & K403332 & https://doi.org/10.6084/m9.figshare. 12605915 \\
\hline Libellulidae & Agrionoptera longitudinalis & M & $\mathrm{AM}$ & K299513 & https://doi.org/10.6084/m9.figshare.12605915 \\
\hline Aeshnidae & Agyrtacantha dirupta & $\mathrm{F}$ & QM & $\mathrm{T} 172175$ & https://doi.org/10.6084/m9.figshare. 12605912 \\
\hline Aeshnidae & Anaciaeschna jaspidea & $\mathrm{F}$ & AM & K281261 & https://doi.org/10.6084/m9.figshare. 12605906 \\
\hline Aeshnidae & Anaciaeschna jaspidea & M & $\mathrm{AM}$ & K305456 & https://doi.org/10.6084/m9.figshare.12605906 \\
\hline Aeshnidae & Anax georgius & M & ANIC & $7-010006$ & https://doi.org/10.6084/m9.figshare. 12605897 \\
\hline Aeshnidae & Anax gibbosulus & $\mathrm{F}$ & $\mathrm{AM}$ & K305449 & https://doi.org/10.6084/m9.figshare. 12605894 \\
\hline Aeshnidae & Anax gibbosulus & M & $\mathrm{AM}$ & K305708 & https://doi.org/10.6084/m9.figshare.12605894 \\
\hline Aeshnidae & Anax guttatus & $\mathrm{F}$ & $\mathrm{AM}$ & K289989 & https://doi.org/10.6084/m9.figshare. 12605888 \\
\hline Aeshnidae & Anax guttatus & M & $\mathrm{AM}$ & K305455 & https://doi.org/10.6084/m9.figshare. 12605888 \\
\hline Aeshnidae & Anax papuensis & $\mathrm{F}$ & $\mathrm{AM}$ & K337585 & https://doi.org/10.6084/m9.figshare. 12605882 \\
\hline Aeshnidae & Anax papuensis & M & $\mathrm{AM}$ & K305671 & https://doi.org/10.6084/m9.figshare. 12605882 \\
\hline Gomphidae & Antipodogomphus acolythus & $\mathrm{F}$ & $\mathrm{AM}$ & K305687 & https://doi.org/10.6084/m9.figshare. 12605873 \\
\hline Gomphidae & Antipodogomphus acolythus & M & $\mathrm{AM}$ & K456201 & https://doi.org/10.6084/m9.figshare. 12605873 \\
\hline Gomphidae & Antipodogomphus dentosus & $\mathrm{F}$ & ANIC & $7-005863$ & https://doi.org/10.6084/m9.figshare. 12605864 \\
\hline Gomphidae & Antipodogomphus dentosus & M & ANIC & $7-005862$ & https://doi.org/10.6084/m9.figshare. 12605864 \\
\hline Gomphidae & Antipodogomphus edentulus & $\mathrm{F}$ & ANIC & $7-005864$ & https://doi.org/10.6084/m9.figshare. 12605858 \\
\hline Gomphidae & Antipodogomphus hodgkini & $\mathrm{F}$ & ANIC & $7-005851$ & https://doi.org/10.6084/m9.figshare. 12605852 \\
\hline Gomphidae & Antipodogomphus hodgkini & M & ANIC & $7-005850$ & https://doi.org/10.6084/m9.figshare. 12605852 \\
\hline Gomphidae & Antipodogomphus neophytus & $\mathrm{F}$ & ANIC & unregistered & https://doi.org/10.6084/m9.figshare. 12605849 \\
\hline Gomphidae & Antipodogomphus neophytus & M & ANIC & $7-005846$ & https://doi.org/10.6084/m9.figshare.12605849 \\
\hline Gomphidae & Antipodogomphus proselythus & $\mathrm{F}$ & $\mathrm{AM}$ & K403324 & https://doi.org/10.6084/m9.figshare. 12605810 \\
\hline Gomphidae & Antipodogomphus proselythus & M & $\mathrm{AM}$ & K403325 & https://doi.org/10.6084/m9.figshare. 12605810 \\
\hline Telephlebiidae & Antipodophlebia asthenes & $\mathrm{F}$ & $\mathrm{AM}$ & K300809 & https://doi.org/10.6084/m9.figshare. 12605807 \\
\hline Telephlebiidae & Antipodophlebia asthenes & M & $\mathrm{AM}$ & K300810 & https://doi.org/10.6084/m9.figshare. 12605807 \\
\hline Austrocorduliidae & Apocordulia macrops & $\mathrm{F}$ & ANIC & unregistered & https://doi.org/10.6084/m9.figshare. 12605801 \\
\hline Austrocorduliidae & Apocordulia macrops & $\mathrm{M}$ & ANIC & $7-010455$ & https://doi.org/10.6084/m9.figshare. 12605801 \\
\hline Gomphomacromiidae & Archaeophya adamsi & $\mathrm{F}$ & ANIC & $7-010270$ & https://doi.org/10.6084/m9.figshare. 12605798 \\
\hline Gomphomacromiidae & Archaeophya adamsi & M & $\mathrm{AM}$ & K259786 & https://doi.org/10.6084/m9.figshare. 12605798 \\
\hline Gomphomacromiidae & Archaeophya magnifica & $\mathrm{F}$ & ANIC & $7-010273$ & https://doi.org/10.6084/m9.figshare. 12605792 \\
\hline Gomphomacromiidae & Archaeophya magnifica & M & $\mathrm{AM}$ & K281178 & https://doi.org/10.6084/m9.figshare. 12605792 \\
\hline Synthemistidae & Archaeosynthemis leachii & $\mathrm{F}$ & $\mathrm{AM}$ & K299902 & https://doi.org/10.6084/m9.figshare. 12605789 \\
\hline Synthemistidae & Archaeosynthemis leachii & M & $\mathrm{AM}$ & K337673 & https://doi.org/10.6084/m9.figshare.12605789 \\
\hline Synthemistidae & Archaeosynthemis occidentalis & $\mathrm{F}$ & ANIC & $7-010976$ & https://doi.org/10.6084/m9.figshare. 12605783 \\
\hline Synthemistidae & Archaeosynthemis occidentalis & M & $\mathrm{AM}$ & K337680 & https://doi.org/10.6084/m9.figshare. 12605783 \\
\hline Synthemistidae & Archaeosynthemis orientalis & $\mathrm{F}$ & $\mathrm{AM}$ & K299886 & https://doi.org/10.6084/m9.figshare. 12605777 \\
\hline Synthemistidae & Archaeosynthemis orientalis & M & $\mathrm{AM}$ & K299892 & https://doi.org/10.6084/m9.figshare. 12605777 \\
\hline Synthemistidae & Archaeosynthemis spiniger & $\mathrm{F}$ & ANIC & $7-011011$ & https://doi.org/10.6084/m9.figshare. 12605756 \\
\hline Synthemistidae & Archaeosynthemis spiniger & M & WAM & WAM44318 & https://doi.org/10.6084/m9.figshare. 12605756 \\
\hline Megapodagrionidae & Archiargiolestes parvulus & $\mathrm{F}$ & ANIC & $7-004674$ & https://doi.org/10.6084/m9.figshare. 12597659 \\
\hline Megapodagrionidae & Archiargiolestes parvulus & $\mathrm{M}$ & ANIC & $7-004658$ & https://doi.org/10.6084/m9.figshare. 12597659 \\
\hline Megapodagrionidae & Archiargiolestes pusillissimus & $\mathrm{F}$ & ANIC & $7-004609$ & https://doi.org/10.6084/m9.figshare. 12597656 \\
\hline Megapodagrionidae & Archiargiolestes pusillissimus & M & ANIC & $7-004614$ & https://doi.org/10.6084/m9.figshare. 12597656 \\
\hline Megapodagrionidae & Archiargiolestes pusillus & $\mathrm{F}$ & ANIC & $7-004465$ & https://doi.org/10.6084/m9.figshare. 12597653 \\
\hline Megapodagrionidae & Archiargiolestes pusillus & M & $\mathrm{AM}$ & K301434 & https://doi.org/10.6084/m9.figshare. 12597653 \\
\hline
\end{tabular}




\begin{tabular}{|c|c|c|c|c|c|}
\hline family & species & $\operatorname{sex}$ & Coll. & Coll.Reg. & figshare DOI for wing images \\
\hline Coenagrionidae & Archibasis mimetes & $\mathrm{F}$ & ANIC & $7-000014$ & https://doi.org/10.6084/m9.figshare. 12597650 \\
\hline Coenagrionidae & Archibasis mimetes & M & $\mathrm{AM}$ & K301186 & https://doi.org/10.6084/m9.figshare. 12597650 \\
\hline Austropetaliidae & Archipetalia auriculata & $\mathrm{F}$ & $\mathrm{AM}$ & K305485 & https://doi.org/10.6084/m9.figshare. 12605750 \\
\hline Austropetaliidae & Archipetalia auriculata & M & $\mathrm{AM}$ & K305667 & https://doi.org/10.6084/m9.figshare. 12605750 \\
\hline Coenagrionidae & Argiocnemis rubescens & $\mathrm{F}$ & $\mathrm{AM}$ & K301177 & https://doi.org/10.6084/m9.figshare.12597647 \\
\hline Coenagrionidae & Argiocnemis rubescens & M & $\mathrm{AM}$ & K301173 & https://doi.org/10.6084/m9.figshare. 12597647 \\
\hline Gomphidae & Armagomphus armiger & $\mathrm{F}$ & ANIC & $7-006033$ & https://doi.org/10.6084/m9.figshare.12605747 \\
\hline Gomphidae & Armagomphus armiger & M & ANIC & $7-006032$ & https://doi.org/10.6084/m9.figshare. 12605747 \\
\hline Telephlebiidae & Austroaeschna anacantha & $\mathrm{F}$ & $\mathrm{AM}$ & K300806 & https://doi.org/10.6084/m9.figshare. 12605741 \\
\hline Telephlebiidae & Austroaeschna anacantha & M & $\mathrm{AM}$ & K337714 & https://doi.org/10.6084/m9.figshare.12605741 \\
\hline Telephlebiidae & Austroaeschna atrata & $\mathrm{F}$ & $\mathrm{AM}$ & K289915 & https://doi.org/10.6084/m9.figshare. 12605732 \\
\hline Telephlebiidae & Austroaeschna atrata & M & $\mathrm{AM}$ & K300795 & https://doi.org/10.6084/m9.figshare.12605732 \\
\hline Telephlebiidae & Austroaeschna christine & M & ANIC & $7-007569$ & https://doi.org/10.6084/m9.figshare.12605666 \\
\hline Telephlebiidae & Austroaeschna cooloola & $\mathrm{F}$ & $\mathrm{AM}$ & K403340 & https://doi.org/10.6084/m9.figshare. 12605657 \\
\hline Telephlebiidae & Austroaeschna cooloola & M & $\mathrm{AM}$ & K300794 & https://doi.org/10.6084/m9.figshare. 12605657 \\
\hline Telephlebiidae & Austroaeschna eungella & $\mathrm{F}$ & ANIC & $7-009489$ & https://doi.org/10.6084/m9.figshare. 12605648 \\
\hline Telephlebiidae & Austroaeschna eungella & M & QM & $\mathrm{T} 175882$ & https://doi.org/10.6084/m9.figshare. 12605648 \\
\hline Telephlebiidae & Austroaeschna flavomaculata & $\mathrm{F}$ & $\mathrm{AM}$ & K300791 & https://doi.org/10.6084/m9.figshare. 12605633 \\
\hline Telephlebiidae & Austroaeschna flavomaculata & M & $\mathrm{AM}$ & K300782 & https://doi.org/10.6084/m9.figshare. 12605633 \\
\hline Telephlebiidae & Austroaeschna hardyi & $\mathrm{F}$ & $\mathrm{AM}$ & K300750 & https://doi.org/10.6084/m9.figshare. 12605609 \\
\hline Telephlebiidae & Austroaeschna hardyi & M & $\mathrm{AM}$ & K300752 & https://doi.org/10.6084/m9.figshare. 12605609 \\
\hline Telephlebiidae & Austroaeschna inermis & $\mathrm{F}$ & $\mathrm{AM}$ & K300256 & https://doi.org/10.6084/m9.figshare. 12605600 \\
\hline Telephlebiidae & Austroaeschna inermis & M & $\mathrm{AM}$ & K300253 & https://doi.org/10.6084/m9.figshare. 12605600 \\
\hline Telephlebiidae & Austroaeschna ingrid & $\mathrm{F}$ & $\mathrm{AM}$ & K300737 & https://doi.org/10.6084/m9.figshare. 12605588 \\
\hline Telephlebiidae & Austroaeschna ingrid & M & $\mathrm{AM}$ & K300740 & https://doi.org/10.6084/m9.figshare. 12605588 \\
\hline Telephlebiidae & Austroaeschna muelleri & $\mathrm{F}$ & $\mathrm{AM}$ & K300907 & https://doi.org/10.6084/m9.figshare. 12605516 \\
\hline Telephlebiidae & Austroaeschna muelleri & M & $\mathrm{AM}$ & K300906 & https://doi.org/10.6084/m9.figshare. 12605516 \\
\hline Telephlebiidae & Austroaeschna multipunctata & $\mathrm{F}$ & $\mathrm{AM}$ & K300886 & https://doi.org/10.6084/m9.figshare. 12605507 \\
\hline Telephlebiidae & Austroaeschna multipunctata & M & $\mathrm{AM}$ & K300861 & https://doi.org/10.6084/m9.figshare. 12605507 \\
\hline Telephlebiidae & Austroaeschna obscura & $\mathrm{F}$ & $\mathrm{AM}$ & K300829 & https://doi.org/10.6084/m9.figshare. 12601517 \\
\hline Telephlebiidae & Austroaeschna obscura & M & $\mathrm{AM}$ & K300826 & https://doi.org/10.6084/m9.figshare. 12601517 \\
\hline Telephlebiidae & Austroaeschna parvistigma & $\mathrm{F}$ & $\mathrm{AM}$ & K300817 & https://doi.org/10.6084/m9.figshare. 12601508 \\
\hline Telephlebiidae & Austroaeschna parvistigma & $\mathrm{M}$ & $\mathrm{AM}$ & K300823 & https://doi.org/10.6084/m9.figshare. 12601508 \\
\hline Telephlebiidae & Austroaeschna pinheyi & $\mathrm{F}$ & $\mathrm{AM}$ & K305676 & https://doi.org/10.6084/m9.figshare. 12601505 \\
\hline Telephlebiidae & Austroaeschna pinheyi & M & $\mathrm{AM}$ & K305675 & https://doi.org/10.6084/m9.figshare. 12601505 \\
\hline Telephlebiidae & Austroaeschna pulchra & $\mathrm{F}$ & $\mathrm{AM}$ & K301002 & https://doi.org/10.6084/m9.figshare. 12601499 \\
\hline Telephlebiidae & Austroaeschna pulchra & M & $\mathrm{AM}$ & K337715 & https://doi.org/10.6084/m9.figshare.12601499 \\
\hline Telephlebiidae & Austroaeschna sigma & $\mathrm{F}$ & $\mathrm{AM}$ & K300979 & https://doi.org/10.6084/m9.figshare. 12601496 \\
\hline Telephlebiidae & Austroaeschna sigma & M & $\mathrm{AM}$ & K300966 & https://doi.org/10.6084/m9.figshare. 12601496 \\
\hline Telephlebiidae & Austroaeschna speciosa & $\mathrm{F}$ & $\mathrm{AM}$ & K289859 & https://doi.org/10.6084/m9.figshare. 12601493 \\
\hline Telephlebiidae & Austroaeschna speciosa & M & ANIC & $7-006781$ & https://doi.org/10.6084/m9.figshare. 12601493 \\
\hline Telephlebiidae & Austroaeschna subapicalis & $\mathrm{F}$ & $\mathrm{AM}$ & K300934 & https://doi.org/10.6084/m9.figshare. 12601478 \\
\hline Telephlebiidae & Austroaeschna subapicalis & M & $\mathrm{AM}$ & K300960 & https://doi.org/10.6084/m9.figshare. 12601478 \\
\hline Telephlebiidae & Austroaeschna tasmanica & $\mathrm{F}$ & QM & $\mathrm{T} 172855$ & https://doi.org/10.6084/m9.figshare. 12601475 \\
\hline Telephlebiidae & Austroaeschna tasmanica & M & AM & K299384 & https://doi.org/10.6084/m9.figshare. 12601475 \\
\hline Telephlebiidae & Austroaeschna unicornis & $\mathrm{F}$ & $\mathrm{AM}$ & K299376 & https://doi.org/10.6084/m9.figshare. 12601472 \\
\hline Telephlebiidae & Austroaeschna unicornis & M & $\mathrm{AM}$ & K299370 & https://doi.org/10.6084/m9.figshare. 12601472 \\
\hline Coenagrionidae & Austroagrion cyane & $\mathrm{F}$ & ANIC & $7-001537$ & https://doi.org/10.6084/m9.figshare. 12597644 \\
\hline Coenagrionidae & Austroagrion cyane & M & ANIC & $7-001526$ & https://doi.org/10.6084/m9.figshare. 12597644 \\
\hline Coenagrionidae & Austroagrion exclamationis & $\mathrm{F}$ & $\mathrm{AM}$ & K301162 & https://doi.org/10.6084/m9.figshare.12597641 \\
\hline Coenagrionidae & Austroagrion exclamationis & M & $\mathrm{AM}$ & K301160 & https://doi.org/10.6084/m9.figshare.12597641 \\
\hline Coenagrionidae & Austroagrion pindrina & $\mathrm{F}$ & ANIC & $7-001109$ & https://doi.org/10.6084/m9.figshare. 12597638 \\
\hline Coenagrionidae & Austroagrion pindrina & M & $\mathrm{AM}$ & K301157 & https://doi.org/10.6084/m9.figshare. 12597638 \\
\hline Coenagrionidae & Austroagrion watsoni & $\mathrm{F}$ & $\mathrm{AM}$ & K301149 & https://doi.org/10.6084/m9.figshare. 12597635 \\
\hline Coenagrionidae & Austroagrion watsoni & M & $\mathrm{AM}$ & K337764 & https://doi.org/10.6084/m9.figshare. 12597635 \\
\hline Megapodagrionidae & Austroargiolestes alpinus & $\mathrm{F}$ & ANIC & $7-009196$ & https://doi.org/10.6084/m9.figshare. 12597632 \\
\hline Megapodagrionidae & Austroargiolestes alpinus & M & ANIC & $7-009190$ & https://doi.org/10.6084/m9.figshare.12597632 \\
\hline Megapodagrionidae & Austroargiolestes amabilis & $\mathrm{F}$ & $\mathrm{AM}$ & K302004 & https://doi.org/10.6084/m9.figshare. 12597626 \\
\hline Megapodagrionidae & Austroargiolestes amabilis & M & $\mathrm{AM}$ & K302014 & https://doi.org/10.6084/m9.figshare. 12597626 \\
\hline Megapodagrionidae & Austroargiolestes aureus & $\mathrm{F}$ & $\mathrm{AM}$ & K302001 & https://doi.org/10.6084/m9.figshare.12597623 \\
\hline Megapodagrionidae & Austroargiolestes aureus & M & $\mathrm{AM}$ & K301989 & https://doi.org/10.6084/m9.figshare. 12597623 \\
\hline Megapodagrionidae & Austroargiolestes brookhousei & $\mathrm{F}$ & $\mathrm{AM}$ & K301983 & https://doi.org/10.6084/m9.figshare. 12597620 \\
\hline Megapodagrionidae & Austroargiolestes brookhousei & M & $\mathrm{AM}$ & K301985 & https://doi.org/10.6084/m9.figshare. 12597620 \\
\hline Megapodagrionidae & Austroargiolestes calcaris & $\mathrm{F}$ & $\mathrm{AM}$ & K301976 & https://doi.org/10.6084/m9.figshare. 12597614 \\
\hline Megapodagrionidae & Austroargiolestes calcaris & M & $\mathrm{AM}$ & K301971 & https://doi.org/10.6084/m9.figshare. 12597614 \\
\hline Megapodagrionidae & Austroargiolestes christine & $\mathrm{F}$ & $\mathrm{AM}$ & K301955 & https://doi.org/10.6084/m9.figshare. 12597605 \\
\hline Megapodagrionidae & Austroargiolestes christine & M & $\mathrm{AM}$ & K301950 & https://doi.org/10.6084/m9.figshare. 12597605 \\
\hline Megapodagrionidae & Austroargiolestes chrysoides & $\mathrm{F}$ & ANIC & $7-004958$ & https://doi.org/10.6084/m9.figshare. 12597602 \\
\hline Megapodagrionidae & Austroargiolestes chrysoides & M & ANIC & $7-009114$ & https://doi.org/10.6084/m9.figshare. 12597602 \\
\hline Megapodagrionidae & Austroargiolestes elke & $\mathrm{F}$ & ANIC & $7-005017$ & https://doi.org/10.6084/m9.figshare.12597593 \\
\hline Megapodagrionidae & Austroargiolestes elke & M & QM & T192479 & https://doi.org/10.6084/m9.figshare. 12597593 \\
\hline Megapodagrionidae & Austroargiolestes icteromelas & $\mathrm{F}$ & AM & K301926 & https://doi.org/10.6084/m9.figshare. 12597587 \\
\hline Megapodagrionidae & Austroargiolestes icteromelas & M & $\mathrm{AM}$ & K337843 & https://doi.org/10.6084/m9.figshare. 12597587 \\
\hline Megapodagrionidae & Austroargiolestes isabellae & $\mathrm{F}$ & $\mathrm{AM}$ & K301847 & https://doi.org/10.6084/m9.figshare. 12597578 \\
\hline Megapodagrionidae & Austroargiolestes isabellae & M & $\mathrm{AM}$ & K337851 & https://doi.org/10.6084/m9.figshare. 12597578 \\
\hline Coenagrionidae & Austrocnemis maccullochi & $\mathrm{F}$ & $\mathrm{AM}$ & K301143 & https://doi.org/10.6084/m9.figshare.12597569 \\
\hline Coenagrionidae & Austrocnemis maccullochi & M & AM & K301134 & https://doi.org/10.6084/m9.figshare.12597569 \\
\hline Coenagrionidae & Austrocnemis obscura & $\mathrm{F}$ & ANIC & unregistered & https://doi.org/10.6084/m9.figshare. 12597563 \\
\hline Coenagrionidae & Austrocnemis splendida & $\mathrm{F}$ & $\mathrm{AM}$ & K301127 & https://doi.org/10.6084/m9.figshare.12597551 \\
\hline Coenagrionidae & Austrocnemis splendida & M & AM & K301116 & https://doi.org/10.6084/m9.figshare.12597551 \\
\hline
\end{tabular}




\begin{tabular}{|c|c|c|c|c|c|}
\hline family & species & sex & Coll. & Coll.Reg. & figshare DOI for wing images \\
\hline Austrocorduliidae & Austrocordulia leonardi & $\mathrm{F}$ & $\mathrm{AM}$ & K259788 & https://doi.org/10.6084/m9.figshare.12601433 \\
\hline Austrocorduliidae & Austrocordulia leonardi & M & $\mathrm{AM}$ & K300186 & https://doi.org/10.6084/m9.figshare. 12601433 \\
\hline Austrocorduliidae & Austrocordulia refracta & $\mathrm{F}$ & AM & K300178 & https://doi.org/10.6084/m9.figshare.12601427 \\
\hline Austrocorduliidae & Austrocordulia refracta & M & $\mathrm{AM}$ & K300177 & https://doi.org/10.6084/m9.figshare.12601427 \\
\hline Austrocorduliidae & Austrocordulia territoria & $\mathrm{F}$ & ANIC & $7-010447$ & https://doi.org/10.6084/m9.figshare.12601421 \\
\hline Austrocorduliidae & Austrocordulia territoria & M & ANIC & $7-010454$ & https://doi.org/10.6084/m9.figshare.12601421 \\
\hline Gomphidae & Austrogomphus amphiclitus & $\mathrm{F}$ & $\mathrm{AM}$ & K302105 & https://doi.org/10.6084/m9.figshare. 12601418 \\
\hline Gomphidae & Austrogomphus amphiclitus & M & AM & K302086 & https://doi.org/10.6084/m9.figshare. 12601418 \\
\hline Gomphidae & Austrogomphus angelorum & $\mathrm{F}$ & $\mathrm{AM}$ & K456021 & https://doi.org/10.6084/m9.figshare.12601403 \\
\hline Gomphidae & Austrogomphus angelorum & M & ANIC & $7-005239$ & https://doi.org/10.6084/m9.figshare. 12601403 \\
\hline Gomphidae & Austrogomphus arbustorum & $\mathrm{F}$ & $\mathrm{AM}$ & K403328 & https://doi.org/10.6084/m9.figshare. 12601388 \\
\hline Gomphidae & Austrogomphus arbustorum & M & $\mathrm{AM}$ & K403364 & https://doi.org/10.6084/m9.figshare. 12601388 \\
\hline Gomphidae & Austrogomphus australis & $\mathrm{F}$ & $\mathrm{AM}$ & K305033 & https://doi.org/10.6084/m9.figshare.12601355 \\
\hline Gomphidae & Austrogomphus australis & M & $\mathrm{AM}$ & K305035 & https://doi.org/10.6084/m9.figshare. 12601355 \\
\hline Gomphidae & Austrogomphus bifurcatus & $\mathrm{F}$ & $\mathrm{AM}$ & K305684 & https://doi.org/10.6084/m9.figshare. 12601340 \\
\hline Gomphidae & Austrogomphus bifurcatus & M & $\mathrm{AM}$ & K305682 & https://doi.org/10.6084/m9.figshare. 12601340 \\
\hline Gomphidae & Austrogomphus collaris & $\mathrm{F}$ & $\mathrm{AM}$ & K337605 & https://doi.org/10.6084/m9.figshare. 12601328 \\
\hline Gomphidae & Austrogomphus collaris & M & $\mathrm{AM}$ & K403327 & https://doi.org/10.6084/m9.figshare. 12601328 \\
\hline Gomphidae & Austrogomphus cornutus & $\mathrm{F}$ & $\mathrm{AM}$ & K302079 & https://doi.org/10.6084/m9.figshare.12601304 \\
\hline Gomphidae & Austrogomphus cornutus & M & $\mathrm{AM}$ & K302074 & https://doi.org/10.6084/m9.figshare. 12601304 \\
\hline Gomphidae & Austrogomphus divaricatus & $\mathrm{F}$ & $\mathrm{AM}$ & K304995 & https://doi.org/10.6084/m9.figshare.12601277 \\
\hline Gomphidae & Austrogomphus divaricatus & M & $\mathrm{AM}$ & K305689 & https://doi.org/10.6084/m9.figshare. 12601277 \\
\hline Gomphidae & Austrogomphus doddi & $\mathrm{F}$ & $\mathrm{AM}$ & K302071 & https://doi.org/10.6084/m9.figshare. 12601247 \\
\hline Gomphidae & Austrogomphus doddi & M & AM & K403329 & https://doi.org/10.6084/m9.figshare.12601247 \\
\hline Gomphidae & Austrogomphus gordoni & $\mathrm{F}$ & ANIC & $7-005644$ & https://doi.org/10.6084/m9.figshare.12601214 \\
\hline Gomphidae & Austrogomphus gordoni & M & $\mathrm{AM}$ & K403326 & https://doi.org/10.6084/m9.figshare. 12601214 \\
\hline Gomphidae & Austrogomphus guerini & $\mathrm{F}$ & $\mathrm{AM}$ & K304978 & https://doi.org/10.6084/m9.figshare. 12601208 \\
\hline Gomphidae & Austrogomphus guerini & M & $\mathrm{AM}$ & K304967 & https://doi.org/10.6084/m9.figshare. 12601208 \\
\hline Gomphidae & Austrogomphus mjobergi & $\mathrm{F}$ & $\mathrm{AM}$ & K304964 & https://doi.org/10.6084/m9.figshare.12601187 \\
\hline Gomphidae & Austrogomphus mjobergi & M & $\mathrm{AM}$ & K403330 & https://doi.org/10.6084/m9.figshare. 12601187 \\
\hline Gomphidae & Austrogomphus mouldsorum & $\mathrm{F}$ & $\mathrm{AM}$ & K292022 & https://doi.org/10.6084/m9.figshare.12601115 \\
\hline Gomphidae & Austrogomphus ochraceus & $\mathrm{F}$ & AM & K337616 & https://doi.org/10.6084/m9.figshare. 12601079 \\
\hline Gomphidae & Austrogomphus ochraceus & M & AM & K302139 & https://doi.org/10.6084/m9.figshare.12601079 \\
\hline Gomphidae & Austrogomphus praeruptus & $\mathrm{F}$ & $\mathrm{AM}$ & K304966 & https://doi.org/10.6084/m9.figshare. 12601043 \\
\hline Gomphidae & Austrogomphus praeruptus & M & $\mathrm{AM}$ & K304965 & https://doi.org/10.6084/m9.figshare.12601043 \\
\hline Gomphidae & Austrogomphus prasinus & $\mathrm{F}$ & $\mathrm{AM}$ & K305012 & https://doi.org/10.6084/m9.figshare.12601037 \\
\hline Gomphidae & Austrogomphus prasinus & M & $\mathrm{AM}$ & K305016 & https://doi.org/10.6084/m9.figshare. 12601037 \\
\hline Gomphidae & Austrogomphus turneri & $\mathrm{F}$ & $\mathrm{AM}$ & K302116 & https://doi.org/10.6084/m9.figshare. 12601034 \\
\hline Gomphidae & Austrogomphus turneri & M & AM & K302115 & https://doi.org/10.6084/m9.figshare. 12601034 \\
\hline Aeshnidae & Austrogynacantha heterogena & $\mathrm{F}$ & $\mathrm{AM}$ & K305438 & https://doi.org/10.6084/m9.figshare. 12601031 \\
\hline Aeshnidae & Austrogynacantha heterogena & M & $\mathrm{AM}$ & K305443 & https://doi.org/10.6084/m9.figshare. 12601031 \\
\hline Lestidae & Austrolestes aleison & $\mathrm{F}$ & $\mathrm{AM}$ & K403349 & https://doi.org/10.6084/m9.figshare. 12597548 \\
\hline Lestidae & Austrolestes aleison & M & $\mathrm{AM}$ & K403348 & https://doi.org/10.6084/m9.figshare.12597548 \\
\hline Lestidae & Austrolestes analis & $\mathrm{F}$ & $\mathrm{AM}$ & K301547 & https://doi.org/10.6084/m9.figshare. 12597545 \\
\hline Lestidae & Austrolestes analis & $\mathrm{M}$ & $\mathrm{AM}$ & K337808 & https://doi.org/10.6084/m9.figshare.12597545 \\
\hline Lestidae & Austrolestes annulosus & $\mathrm{F}$ & $\mathrm{AM}$ & K301536 & https://doi.org/10.6084/m9.figshare.12597539 \\
\hline Lestidae & Austrolestes annulosus & M & $\mathrm{AM}$ & K337813 & https://doi.org/10.6084/m9.figshare.12597539 \\
\hline Lestidae & Austrolestes aridus & $\mathrm{F}$ & $\mathrm{AM}$ & K301530 & https://doi.org/10.6084/m9.figshare. 12597530 \\
\hline Lestidae & Austrolestes aridus & M & $\mathrm{AM}$ & K301529 & https://doi.org/10.6084/m9.figshare. 12597530 \\
\hline Lestidae & Austrolestes cingulatus & $\mathrm{F}$ & $\mathrm{AM}$ & K301524 & https://doi.org/10.6084/m9.figshare. 12597527 \\
\hline Lestidae & Austrolestes cingulatus & M & $\mathrm{AM}$ & K337814 & https://doi.org/10.6084/m9.figshare.12597527 \\
\hline Lestidae & Austrolestes insularis & $\mathrm{F}$ & $\mathrm{AM}$ & K301514 & https://doi.org/10.6084/m9.figshare. 12597524 \\
\hline Lestidae & Austrolestes insularis & M & $\mathrm{AM}$ & K301512 & https://doi.org/10.6084/m9.figshare.12597524 \\
\hline Lestidae & Austrolestes io & $\mathrm{F}$ & $\mathrm{AM}$ & K403351 & https://doi.org/10.6084/m9.figshare. 12597518 \\
\hline Lestidae & Austrolestes io & $\mathrm{M}$ & $\mathrm{AM}$ & K403350 & https://doi.org/10.6084/m9.figshare. 12597518 \\
\hline Lestidae & Austrolestes leda & $\mathrm{F}$ & $\mathrm{AM}$ & K301498 & https://doi.org/10.6084/m9.figshare.12597515 \\
\hline Lestidae & Austrolestes leda & M & $\mathrm{AM}$ & K301497 & https://doi.org/10.6084/m9.figshare.12597515 \\
\hline Lestidae & Austrolestes minjerriba & $\mathrm{F}$ & $\mathrm{AM}$ & K302063 & https://doi.org/10.6084/m9.figshare. 12597509 \\
\hline Lestidae & Austrolestes minjerriba & M & $\mathrm{AM}$ & K305602 & https://doi.org/10.6084/m9.figshare. 12597509 \\
\hline Lestidae & Austrolestes psyche & $\mathrm{F}$ & AM & K302046 & https://doi.org/10.6084/m9.figshare. 12597506 \\
\hline Lestidae & Austrolestes psyche & M & $\mathrm{AM}$ & K302068 & https://doi.org/10.6084/m9.figshare. 12597506 \\
\hline Austropetaliidae & Austropetalia patricia & $\mathrm{F}$ & $\mathrm{AM}$ & K403343 & https://doi.org/10.6084/m9.figshare. 12601019 \\
\hline Austropetaliidae & Austropetalia patricia & M & $\mathrm{AM}$ & K305484 & https://doi.org/10.6084/m9.figshare.12601019 \\
\hline Austropetaliidae & Austropetalia tonyana & $\mathrm{F}$ & ANIC & $7-006152$ & https://doi.org/10.6084/m9.figshare.12601013 \\
\hline Austropetaliidae & Austropetalia tonyana & M & $\mathrm{AM}$ & K305483 & https://doi.org/10.6084/m9.figshare.12601013 \\
\hline Telephlebiidae & Austrophlebia costalis & $\mathrm{F}$ & $\mathrm{AM}$ & K299346 & https://doi.org/10.6084/m9.figshare.12601004 \\
\hline Telephlebiidae & Austrophlebia costalis & M & $\mathrm{AM}$ & K299351 & https://doi.org/10.6084/m9.figshare. 12601004 \\
\hline Telephlebiidae & Austrophlebia subcostalis & $\mathrm{F}$ & ANIC & $7-006318$ & https://doi.org/10.6084/m9.figshare. 12601001 \\
\hline Telephlebiidae & Austrophlebia subcostalis & M & ANIC & $7-009666$ & https://doi.org/10.6084/m9.figshare. 12601001 \\
\hline Austrocorduliidae & Austrophya mystica & $\mathrm{F}$ & $\mathrm{AM}$ & K300168 & https://doi.org/10.6084/m9.figshare. 12600998 \\
\hline Austrocorduliidae & Austrophya mystica & M & $\mathrm{AM}$ & K300167 & https://doi.org/10.6084/m9.figshare. 12600998 \\
\hline Isostictidae & Austrosticta fieldi & $\mathrm{F}$ & ANIC & $7-002795$ & https://doi.org/10.6084/m9.figshare. 12597500 \\
\hline Isostictidae & Austrosticta fieldi & M & ANIC & $7-002791$ & https://doi.org/10.6084/m9.figshare. 12597500 \\
\hline Isostictidae & Austrosticta frater & $\mathrm{F}$ & $\mathrm{AM}$ & K301419 & https://doi.org/10.6084/m9.figshare.12597494 \\
\hline Isostictidae & Austrosticta frater & M & $\mathrm{AM}$ & K301417 & https://doi.org/10.6084/m9.figshare.12597494 \\
\hline Isostictidae & Austrosticta soror & $\mathrm{F}$ & ANIC & $7-002805$ & https://doi.org/10.6084/m9.figshare.12597491 \\
\hline Isostictidae & Austrosticta soror & M & ANIC & $7-002800$ & https://doi.org/10.6084/m9.figshare.12597491 \\
\hline Synthemistidae & Austrosynthemis cyanitincta & $\mathrm{F}$ & $\mathrm{AM}$ & K403345 & https://doi.org/10.6084/m9.figshare. 12600995 \\
\hline Synthemistidae & Austrosynthemis cyanitincta & M & $\mathrm{AM}$ & K337685 & https://doi.org/10.6084/m9.figshare.12600995 \\
\hline Libellulidae & Austrothemis nigrescens & $\mathrm{F}$ & $\mathrm{AM}$ & K299495 & https://doi.org/10.6084/m9.figshare.12600992 \\
\hline
\end{tabular}




\begin{tabular}{|c|c|c|c|c|c|}
\hline family & species & $\operatorname{sex}$ & Coll. & Coll.Reg. & figshare DOI for wing images \\
\hline Libellulidae & Austrothemis nigrescens & M & $\mathrm{AM}$ & K299491 & https://doi.org/10.6084/m9.figshare.12600992 \\
\hline Libellulidae & Brachydiplax denticauda & $\mathrm{F}$ & $\mathrm{AM}$ & K299485 & https://doi.org/10.6084/m9.figshare. 12600965 \\
\hline Libellulidae & Brachydiplax denticauda & M & $\mathrm{AM}$ & K299489 & https://doi.org/10.6084/m9.figshare. 12600965 \\
\hline Libellulidae & Brachydiplax duivenbodei & M & $\mathrm{AM}$ & K403372 & https://doi.org/10.6084/m9.figshare. 12600854 \\
\hline Coenagrionidae & Caliagrion billinghursti & $\mathrm{F}$ & $\mathrm{AM}$ & K301097 & https://doi.org/10.6084/m9.figshare.12597467 \\
\hline Coenagrionidae & Caliagrion billinghursti & M & $\mathrm{AM}$ & K301098 & https://doi.org/10.6084/m9.figshare.12597467 \\
\hline Libellulidae & Camacinia othello & $\mathrm{F}$ & $\mathrm{AM}$ & K403335 & https://doi.org/10.6084/m9.figshare. 12600845 \\
\hline Libellulidae & Camacinia othello & M & $\mathrm{AM}$ & K403334 & https://doi.org/10.6084/m9.figshare. 12600845 \\
\hline Coenagrionidae & Ceriagrion aeruginosum & $\mathrm{F}$ & $\mathrm{AM}$ & K301085 & https://doi.org/10.6084/m9.figshare. 12597443 \\
\hline Coenagrionidae & Ceriagrion aeruginosum & M & $\mathrm{AM}$ & K301087 & https://doi.org/10.6084/m9.figshare.12597443 \\
\hline Synlestidae & Chorismagrion risi & $\mathrm{F}$ & $\mathrm{AM}$ & K301633 & https://doi.org/10.6084/m9.figshare.12597413 \\
\hline Synlestidae & Chorismagrion risi & M & $\mathrm{AM}$ & K301630 & https://doi.org/10.6084/m9.figshare.12597413 \\
\hline Synthemistidae & Choristhemis flavoterminata & $\mathrm{F}$ & $\mathrm{AM}$ & K299909 & https://doi.org/10.6084/m9.figshare. 12600839 \\
\hline Synthemistidae & Choristhemis flavoterminata & M & $\mathrm{AM}$ & K299913 & https://doi.org/10.6084/m9.figshare. 12600839 \\
\hline Coenagrionidae & Coenagrion lyelli & $\mathrm{F}$ & $\mathrm{AM}$ & K301103 & https://doi.org/10.6084/m9.figshare.12592064 \\
\hline Coenagrionidae & Coenagrion lyelli & M & $\mathrm{AM}$ & K301111 & https://doi.org/10.6084/m9.figshare.12592064 \\
\hline Cordulephyidae & Cordulephya bidens & $\mathrm{F}$ & ANIC & $7-010421$ & https://doi.org/10.6084/m9.figshare. 12600836 \\
\hline Cordulephyidae & Cordulephya bidens & M & ANIC & $7-010420$ & https://doi.org/10.6084/m9.figshare. 12600836 \\
\hline Cordulephyidae & Cordulephya divergens & $\mathrm{F}$ & $\mathrm{AM}$ & K259718 & https://doi.org/10.6084/m9.figshare. 12600830 \\
\hline Cordulephyidae & Cordulephya divergens & M & $\mathrm{AM}$ & K259816 & https://doi.org/10.6084/m9.figshare. 12600830 \\
\hline Cordulephyidae & Cordulephya montana & $\mathrm{F}$ & ANIC & $7-010414$ & https://doi.org/10.6084/m9.figshare. 12600827 \\
\hline Cordulephyidae & Cordulephya montana & M & $\mathrm{AM}$ & K305491 & https://doi.org/10.6084/m9.figshare. 12600827 \\
\hline Cordulephyidae & Cordulephya pygmaea & $\mathrm{F}$ & $\mathrm{AM}$ & K403342 & https://doi.org/10.6084/m9.figshare. 12600818 \\
\hline Cordulephyidae & Cordulephya pygmaea & M & $\mathrm{AM}$ & K259814 & https://doi.org/10.6084/m9.figshare. 12600818 \\
\hline Libellulidae & Crocothemis nigrifrons & $\mathrm{F}$ & $\mathrm{AM}$ & K299482 & https://doi.org/10.6084/m9.figshare. 12600812 \\
\hline Libellulidae & Crocothemis nigrifrons & M & $\mathrm{AM}$ & K299480 & https://doi.org/10.6084/m9.figshare. 12600812 \\
\hline Brachytronidae & Dendroaeschna conspersa & $\mathrm{F}$ & $\mathrm{AM}$ & K299431 & https://doi.org/10.6084/m9.figshare.12600809 \\
\hline Brachytronidae & Dendroaeschna conspersa & M & $\mathrm{AM}$ & K299433 & https://doi.org/10.6084/m9.figshare. 12600809 \\
\hline Lestoideidae & Diphlebia coerulescens & $\mathrm{F}$ & $\mathrm{AM}$ & K301750 & https://doi.org/10.6084/m9.figshare. 12592055 \\
\hline Lestoideidae & Diphlebia coerulescens & M & $\mathrm{AM}$ & K301757 & https://doi.org/10.6084/m9.figshare. 12592055 \\
\hline Lestoideidae & Diphlebia euphoeoides & $\mathrm{F}$ & $\mathrm{AM}$ & K301716 & https://doi.org/10.6084/m9.figshare. 12592052 \\
\hline Lestoideidae & Diphlebia euphoeoides & M & $\mathrm{AM}$ & K301723 & https://doi.org/10.6084/m9.figshare.12592052 \\
\hline Lestoideidae & Diphlebia hybridoides & $\mathrm{F}$ & $\mathrm{AM}$ & K301691 & https://doi.org/10.6084/m9.figshare. 12592043 \\
\hline Lestoideidae & Diphlebia hybridoides & M & $\mathrm{AM}$ & K301690 & https://doi.org/10.6084/m9.figshare. 12592043 \\
\hline Lestoideidae & Diphlebia lestoides & $\mathrm{F}$ & $\mathrm{AM}$ & K301684 & https://doi.org/10.6084/m9.figshare. 12592028 \\
\hline Lestoideidae & Diphlebia lestoides & M & $\mathrm{AM}$ & K301685 & https://doi.org/10.6084/m9.figshare. 12592028 \\
\hline Lestoideidae & Diphlebia nymphoides & $\mathrm{F}$ & $\mathrm{AM}$ & K301672 & https://doi.org/10.6084/m9.figshare.12592019 \\
\hline Lestoideidae & Diphlebia nymphoides & M & $\mathrm{AM}$ & K301673 & https://doi.org/10.6084/m9.figshare.12592019 \\
\hline Libellulidae & Diplacodes bipunctata & $\mathrm{F}$ & $\mathrm{AM}$ & K299465 & https://doi.org/10.6084/m9.figshare. 12600791 \\
\hline Libellulidae & Diplacodes bipunctata & M & $\mathrm{AM}$ & K305566 & https://doi.org/10.6084/m9.figshare.12600791 \\
\hline Libellulidae & Diplacodes haematodes & $\mathrm{F}$ & $\mathrm{AM}$ & K337655 & https://doi.org/10.6084/m9.figshare. 12600788 \\
\hline Libellulidae & Diplacodes haematodes & M & $\mathrm{AM}$ & K305549 & https://doi.org/10.6084/m9.figshare. 12600788 \\
\hline Libellulidae & Diplacodes melanopsis & $\mathrm{F}$ & $\mathrm{AM}$ & K299523 & https://doi.org/10.6084/m9.figshare. 12600779 \\
\hline Libellulidae & Diplacodes melanopsis & M & $\mathrm{AM}$ & K299525 & https://doi.org/10.6084/m9.figshare. 12600779 \\
\hline Libellulidae & Diplacodes nebulosa & $\mathrm{F}$ & $\mathrm{AM}$ & K403333 & https://doi.org/10.6084/m9.figshare. 12600755 \\
\hline Libellulidae & Diplacodes nebulosa & M & $\mathrm{AM}$ & K299519 & https://doi.org/10.6084/m9.figshare. 12600755 \\
\hline Libellulidae & Diplacodes trivialis & $\mathrm{F}$ & $\mathrm{AM}$ & K299533 & https://doi.org/10.6084/m9.figshare. 12600743 \\
\hline Libellulidae & Diplacodes trivialis & M & $\mathrm{AM}$ & K299540 & https://doi.org/10.6084/m9.figshare. 12600743 \\
\hline Telephlebiidae & Dromaeschna forcipata & $\mathrm{F}$ & $\mathrm{AM}$ & K337753 & https://doi.org/10.6084/m9.figshare. 12600689 \\
\hline Telephlebiidae & Dromaeschna forcipata & M & $\mathrm{AM}$ & K300759 & https://doi.org/10.6084/m9.figshare. 12600689 \\
\hline Telephlebiidae & Dromaeschna weiskei & $\mathrm{F}$ & $\mathrm{AM}$ & K299368 & https://doi.org/10.6084/m9.figshare. 12600632 \\
\hline Telephlebiidae & Dromaeschna weiskei & M & $\mathrm{AM}$ & K301062 & https://doi.org/10.6084/m9.figshare. 12600632 \\
\hline Synlestidae & Episynlestes albicauda & $\mathrm{F}$ & $\mathrm{AM}$ & K301625 & https://doi.org/10.6084/m9.figshare. 12592016 \\
\hline Synlestidae & Episynlestes albicauda & M & $\mathrm{AM}$ & K301627 & https://doi.org/10.6084/m9.figshare. 12592016 \\
\hline Synlestidae & Episynlestes cristatus & $\mathrm{F}$ & $\mathrm{AM}$ & K301616 & https://doi.org/10.6084/m9.figshare.12592007 \\
\hline Synlestidae & Episynlestes cristatus & M & $\mathrm{AM}$ & K301617 & https://doi.org/10.6084/m9.figshare.12592007 \\
\hline Synlestidae & Episynlestes intermedius & $\mathrm{F}$ & QM & T192492 & https://doi.org/10.6084/m9.figshare.12592001 \\
\hline Synlestidae & Episynlestes intermedius & M & ANIC & 7-008995 & https://doi.org/10.6084/m9.figshare.12592001 \\
\hline Isostictidae & Eurysticta coolawanyah & $\mathrm{F}$ & ANIC & $7-002757$ & https://doi.org/10.6084/m9.figshare.12591989 \\
\hline Isostictidae & Eurysticta coolawanyah & M & $\mathrm{AM}$ & K301415 & https://doi.org/10.6084/m9.figshare.12591989 \\
\hline Isostictidae & Eurysticta coomalie & $\mathrm{F}$ & ANIC & $7-002727$ & https://doi.org/10.6084/m9.figshare. 12591965 \\
\hline Isostictidae & Eurysticta coomalie & M & ANIC & $7-002727$ & https://doi.org/10.6084/m9.figshare. 12591965 \\
\hline Isostictidae & Eurysticta kununurra & $\mathrm{F}$ & ANIC & $7-002723$ & https://doi.org/10.6084/m9.figshare. 12591956 \\
\hline Isostictidae & Eurysticta kununurra & M & ANIC & $7-002723$ & https://doi.org/10.6084/m9.figshare. 12591956 \\
\hline Synthemistidae & Eusynthemis aurolineata & $\mathrm{F}$ & $\mathrm{AM}$ & K299879 & https://doi.org/10.6084/m9.figshare. 12600584 \\
\hline Synthemistidae & Eusynthemis aurolineata & M & $\mathrm{AM}$ & K299863 & https://doi.org/10.6084/m9.figshare. 12600584 \\
\hline Synthemistidae & Eusynthemis barbarae & $\mathrm{F}$ & $\mathrm{AM}$ & K259713 & https://doi.org/10.6084/m9.figshare. 12600554 \\
\hline Synthemistidae & Eusynthemis barbarae & M & $\mathrm{AM}$ & K292027 & https://doi.org/10.6084/m9.figshare. 12600554 \\
\hline Synthemistidae & Eusynthemis brevistyla & $\mathrm{F}$ & $\mathrm{AM}$ & K299767 & https://doi.org/10.6084/m9.figshare. 12600536 \\
\hline Synthemistidae & Eusynthemis brevistyla & M & $\mathrm{AM}$ & K299763 & https://doi.org/10.6084/m9.figshare. 12600536 \\
\hline Synthemistidae & Eusynthemis deniseae & $\mathrm{F}$ & $\mathrm{AM}$ & K299829 & https://doi.org/10.6084/m9.figshare.12600404 \\
\hline Synthemistidae & Eusynthemis deniseae & $\mathrm{M}$ & $\mathrm{AM}$ & K299828 & https://doi.org/10.6084/m9.figshare. 12600404 \\
\hline Synthemistidae & Eusynthemis guttata & $\mathrm{F}$ & $\mathrm{AM}$ & K299822 & https://doi.org/10.6084/m9.figshare. 12600398 \\
\hline Synthemistidae & Eusynthemis guttata & M & $\mathrm{AM}$ & K299817 & https://doi.org/10.6084/m9.figshare. 12600398 \\
\hline Synthemistidae & Eusynthemis nigra & $\mathrm{F}$ & $\mathrm{AM}$ & K305698 & https://doi.org/10.6084/m9.figshare. 12600389 \\
\hline Synthemistidae & Eusynthemis nigra & M & $\mathrm{AM}$ & K300197 & https://doi.org/10.6084/m9.figshare. 12600389 \\
\hline Synthemistidae & Eusynthemis rentziana & $\mathrm{F}$ & $\mathrm{AM}$ & K299807 & https://doi.org/10.6084/m9.figshare.12600386 \\
\hline Synthemistidae & Eusynthemis rentziana & M & ANIC & $7-010123$ & https://doi.org/10.6084/m9.figshare. 12600386 \\
\hline Synthemistidae & Eusynthemis tenera & $\mathrm{F}$ & ANIC & $7-010114$ & https://doi.org/10.6084/m9.figshare. 12600383 \\
\hline Synthemistidae & Eusynthemis tillyardi & $\mathrm{F}$ & $\mathrm{AM}$ & K299805 & https://doi.org/10.6084/m9.figshare. 12600380 \\
\hline
\end{tabular}




\begin{tabular}{|c|c|c|c|c|c|}
\hline family & species & $\operatorname{sex}$ & Coll. & Coll.Reg. & figshare DOI for wing images \\
\hline Synthemistidae & Eusynthemis tillyardi & M & $\mathrm{AM}$ & K299806 & https://doi.org/10.6084/m9.figshare. 12600380 \\
\hline Synthemistidae & Eusynthemis ursa & M & ANIC & $7-010021$ & https://doi.org/10.6084/m9.figshare.12600374 \\
\hline Synthemistidae & Eusynthemis ursula & M & $\mathrm{AM}$ & K259768 & https://doi.org/10.6084/m9.figshare. 12600371 \\
\hline Synthemistidae & Eusynthemis virgula & $\mathrm{F}$ & $\mathrm{AM}$ & K299792 & https://doi.org/10.6084/m9.figshare. 12600368 \\
\hline Synthemistidae & Eusynthemis virgula & M & $\mathrm{AM}$ & K299787 & https://doi.org/10.6084/m9.figshare. 12600368 \\
\hline Megapodagrionidae & Griseargiolestes albescens & $\mathrm{F}$ & $\mathrm{AM}$ & K301834 & https://doi.org/10.6084/m9.figshare. 12591950 \\
\hline Megapodagrionidae & Griseargiolestes albescens & M & $\mathrm{AM}$ & K301827 & https://doi.org/10.6084/m9.figshare. 12591950 \\
\hline Megapodagrionidae & Griseargiolestes bucki & $\mathrm{F}$ & $\mathrm{AM}$ & K301796 & https://doi.org/10.6084/m9.figshare. 12591944 \\
\hline Megapodagrionidae & Griseargiolestes bucki & M & $\mathrm{AM}$ & K301791 & https://doi.org/10.6084/m9.figshare. 12591944 \\
\hline Megapodagrionidae & Griseargiolestes eboracus & $\mathrm{F}$ & $\mathrm{AM}$ & K301492 & https://doi.org/10.6084/m9.figshare.12591926 \\
\hline Megapodagrionidae & Griseargiolestes eboracus & M & $\mathrm{AM}$ & K301491 & https://doi.org/10.6084/m9.figshare. 12591926 \\
\hline Megapodagrionidae & Griseargiolestes fontanus & $\mathrm{F}$ & ANIC & 7-009109 & https://doi.org/10.6084/m9.figshare.12591914 \\
\hline Megapodagrionidae & Griseargiolestes fontanus & M & ANIC & 7-009116 & https://doi.org/10.6084/m9.figshare.12591914 \\
\hline Megapodagrionidae & Griseargiolestes griseus & $\mathrm{F}$ & $\mathrm{AM}$ & K301474 & https://doi.org/10.6084/m9.figshare. 12591905 \\
\hline Megapodagrionidae & Griseargiolestes griseus & M & $\mathrm{AM}$ & K301478 & https://doi.org/10.6084/m9.figshare. 12591905 \\
\hline Megapodagrionidae & Griseargiolestes intermedius & $\mathrm{F}$ & ANIC & $7-009050$ & https://doi.org/10.6084/m9.figshare. 12591902 \\
\hline Megapodagrionidae & Griseargiolestes intermedius & M & $\mathrm{AM}$ & K301447 & https://doi.org/10.6084/m9.figshare.12591902 \\
\hline Megapodagrionidae & Griseargiolestes metallicus & $\mathrm{F}$ & ANIC & 7-009063 & https://doi.org/10.6084/m9.figshare.12591899 \\
\hline Megapodagrionidae & Griseargiolestes metallicus & M & ANIC & $7-009060$ & https://doi.org/10.6084/m9.figshare.12591899 \\
\hline Aeshnidae & Gynacantha dobsoni & $\mathrm{F}$ & $\mathrm{AM}$ & K305429 & https://doi.org/10.6084/m9.figshare. 12600080 \\
\hline Aeshnidae & Gynacantha dobsoni & M & $\mathrm{AM}$ & K305430 & https://doi.org/10.6084/m9.figshare. 12600080 \\
\hline Aeshnidae & Gynacantha kirbyi & $\mathrm{F}$ & AM & K305422 & https://doi.org/10.6084/m9.figshare. 12600071 \\
\hline Aeshnidae & Gynacantha kirbyi & $\mathrm{M}$ & AM & K305424 & https://doi.org/10.6084/m9.figshare. 12600071 \\
\hline Aeshnidae & Gynacantha mocsaryi & $\mathrm{F}$ & $\mathrm{AM}$ & K305669 & https://doi.org/10.6084/m9.figshare. 12600065 \\
\hline Aeshnidae & Gynacantha mocsaryi & M & $\mathrm{AM}$ & K305421 & https://doi.org/10.6084/m9.figshare. 12600065 \\
\hline Aeshnidae & Gynacantha nourlangie & $\mathrm{F}$ & $\mathrm{AM}$ & K403321 & https://doi.org/10.6084/m9.figshare. 12600059 \\
\hline Aeshnidae & Gynacantha nourlangie & M & $\mathrm{AM}$ & K403320 & https://doi.org/10.6084/m9.figshare. 12600059 \\
\hline Aeshnidae & Gynacantha rosenbergi & $\mathrm{F}$ & $\mathrm{AM}$ & K305408 & https://doi.org/10.6084/m9.figshare. 12600053 \\
\hline Aeshnidae & Gynacantha rosenbergi & M & $\mathrm{AM}$ & K305407 & https://doi.org/10.6084/m9.figshare. 12600053 \\
\hline Corduliidae & Hemicordulia australiae & $\mathrm{F}$ & $\mathrm{AM}$ & K300247 & https://doi.org/10.6084/m9.figshare. 12600047 \\
\hline Corduliidae & Hemicordulia australiae & M & AM & K300250 & https://doi.org/10.6084/m9.figshare. 12600047 \\
\hline Corduliidae & Hemicordulia continentalis & $\mathrm{F}$ & $\mathrm{AM}$ & K300228 & https://doi.org/10.6084/m9.figshare.12600044 \\
\hline Corduliidae & Hemicordulia continentalis & M & $\mathrm{AM}$ & K300227 & https://doi.org/10.6084/m9.figshare. 12600044 \\
\hline Corduliidae & Hemicordulia flava & $\mathrm{F}$ & ANIC & $7-010564$ & https://doi.org/10.6084/m9.figshare. 12600041 \\
\hline Corduliidae & Hemicordulia flava & M & $\mathrm{AM}$ & K300222 & https://doi.org/10.6084/m9.figshare. 12600041 \\
\hline Corduliidae & Hemicordulia intermedia & $\mathrm{F}$ & $\mathrm{AM}$ & K300217 & https://doi.org/10.6084/m9.figshare. 12600032 \\
\hline Corduliidae & Hemicordulia intermedia & M & $\mathrm{AM}$ & K300211 & https://doi.org/10.6084/m9.figshare. 12600032 \\
\hline Corduliidae & Hemicordulia kalliste & $\mathrm{F}$ & ANIC & $7-010571$ & https://doi.org/10.6084/m9.figshare. 12600029 \\
\hline Corduliidae & Hemicordulia kalliste & M & ANIC & $7-010570$ & https://doi.org/10.6084/m9.figshare. 12600029 \\
\hline Corduliidae & Hemicordulia koomina & $\mathrm{F}$ & ANIC & $7-010568$ & https://doi.org/10.6084/m9.figshare. 12600026 \\
\hline Corduliidae & Hemicordulia koomina & M & ANIC & $7-010566$ & https://doi.org/10.6084/m9.figshare. 12600026 \\
\hline Corduliidae & Hemicordulia superba & $\mathrm{F}$ & AM & K305501 & https://doi.org/10.6084/m9.figshare. 12600023 \\
\hline Corduliidae & Hemicordulia superba & M & QM & $\mathrm{T} 181120$ & https://doi.org/10.6084/m9.figshare. 12600023 \\
\hline Corduliidae & Hemicordulia tau & $\mathrm{F}$ & AM & K305494 & https://doi.org/10.6084/m9.figshare. 12600017 \\
\hline Corduliidae & Hemicordulia tau & M & AM & K337597 & https://doi.org/10.6084/m9.figshare. 12600017 \\
\hline Gomphidae & Hemigomphus atratus & M & ANIC & $7-005966$ & https://doi.org/10.6084/m9.figshare. 12600011 \\
\hline Gomphidae & Hemigomphus comitatus & $\mathrm{F}$ & $\mathrm{AM}$ & K305688 & https://doi.org/10.6084/m9.figshare. 12600005 \\
\hline Gomphidae & Hemigomphus comitatus & M & $\mathrm{AM}$ & K305117 & https://doi.org/10.6084/m9.figshare. 12600005 \\
\hline Gomphidae & Hemigomphus cooloola & $\mathrm{F}$ & ANIC & $7-005971$ & https://doi.org/10.6084/m9.figshare. 12600002 \\
\hline Gomphidae & Hemigomphus cooloola & M & ANIC & $7-007445$ & https://doi.org/10.6084/m9.figshare. 12600002 \\
\hline Gomphidae & Hemigomphus gouldii & $\mathrm{F}$ & AM & K305091 & https://doi.org/10.6084/m9.figshare.12599996 \\
\hline Gomphidae & Hemigomphus gouldii & M & $\mathrm{AM}$ & K305087 & https://doi.org/10.6084/m9.figshare. 12599996 \\
\hline Gomphidae & Hemigomphus heteroclytus & $\mathrm{F}$ & $\mathrm{AM}$ & K305063 & https://doi.org/10.6084/m9.figshare. 12599990 \\
\hline Gomphidae & Hemigomphus heteroclytus & M & AM & K305068 & https://doi.org/10.6084/m9.figshare. 12599990 \\
\hline Gomphidae & Hemigomphus magela & $\mathrm{F}$ & ANIC & $7-005995$ & https://doi.org/10.6084/m9.figshare. 12599984 \\
\hline Gomphidae & Hemigomphus magela & $\mathrm{M}$ & ANIC & $7-005993$ & https://doi.org/10.6084/m9.figshare.12599984 \\
\hline Gomphidae & Hemigomphus theischingeri & $\mathrm{F}$ & AM & K403344 & https://doi.org/10.6084/m9.figshare.12599981 \\
\hline Gomphidae & Hemigomphus theischingeri & M & $\mathrm{AM}$ & K302112 & https://doi.org/10.6084/m9.figshare.12599981 \\
\hline Hemiphlebiidae & Hemiphlebia mirabilis & $\mathrm{F}$ & $\mathrm{AM}$ & K280829 & https://doi.org/10.6084/m9.figshare. 12591890 \\
\hline Hemiphlebiidae & Hemiphlebia mirabilis & M & AM & K280832 & https://doi.org/10.6084/m9.figshare. 12591890 \\
\hline Austrocorduliidae & Hesperocordulia berthoudi & $\mathrm{F}$ & AM & K403363 & https://doi.org/10.6084/m9.figshare.12599975 \\
\hline Austrocorduliidae & Hesperocordulia berthoudi & M & AM & K337587 & https://doi.org/10.6084/m9.figshare.12599975 \\
\hline Libellulidae & Huonia melvillensis & M & $\mathrm{AM}$ & K299549 & https://doi.org/10.6084/m9.figshare.12599969 \\
\hline Libellulidae & Hydrobasileus brevistylus & $\mathrm{F}$ & $\mathrm{AM}$ & K299558 & https://doi.org/10.6084/m9.figshare. 12599960 \\
\hline Libellulidae & Hydrobasileus brevistylus & M & $\mathrm{AM}$ & K299554 & https://doi.org/10.6084/m9.figshare. 12599960 \\
\hline Lindeniidae & Ictinogomphus australis & $\mathrm{F}$ & AM & K305045 & https://doi.org/10.6084/m9.figshare. 12599957 \\
\hline Lindeniidae & Ictinogomphus australis & M & AM & K305040 & https://doi.org/10.6084/m9.figshare. 12599957 \\
\hline Lindeniidae & Ictinogomphus dobsoni & $\mathrm{F}$ & AM & K456313 & https://doi.org/10.6084/m9.figshare.12599954 \\
\hline Lindeniidae & Ictinogomphus dobsoni & M & $\mathrm{AM}$ & K305036 & https://doi.org/10.6084/m9.figshare. 12599954 \\
\hline Lindeniidae & Ictinogomphus paulini & M & $\mathrm{AM}$ & K403376 & https://doi.org/10.6084/m9.figshare. 12599948 \\
\hline Lestidae & Indolestes alleni & $\mathrm{F}$ & $\mathrm{AM}$ & K302036 & https://doi.org/10.6084/m9.figshare. 12591884 \\
\hline Lestidae & Indolestes alleni & M & AM & K403347 & https://doi.org/10.6084/m9.figshare.12591884 \\
\hline Lestidae & Indolestes obiri & $\mathrm{F}$ & ANIC & $7-003705$ & https://doi.org/10.6084/m9.figshare.12591881 \\
\hline Lestidae & Indolestes obiri & M & ANIC & $7-003707$ & https://doi.org/10.6084/m9.figshare. 12591881 \\
\hline Lestidae & Indolestes tenuissimus & $\mathrm{F}$ & $\mathrm{AM}$ & K302034 & https://doi.org/10.6084/m9.figshare. 12591878 \\
\hline Lestidae & Indolestes tenuissimus & M & $\mathrm{AM}$ & K302032 & https://doi.org/10.6084/m9.figshare. 12591878 \\
\hline Coenagrionidae & Ischnura aurora & $\mathrm{F}$ & $\mathrm{AM}$ & K301077 & https://doi.org/10.6084/m9.figshare.12591869 \\
\hline Coenagrionidae & Ischnura aurora & M & $\mathrm{AM}$ & K301076 & https://doi.org/10.6084/m9.figshare. 12591869 \\
\hline Coenagrionidae & Ischnura heterosticta & $\mathrm{F}$ & $\mathrm{AM}$ & K305404 & https://doi.org/10.6084/m9.figshare. 12591863 \\
\hline Coenagrionidae & Ischnura heterosticta & M & $\mathrm{AM}$ & K305403 & https://doi.org/10.6084/m9.figshare. 12591863 \\
\hline
\end{tabular}




\begin{tabular}{|c|c|c|c|c|c|}
\hline family & species & $\operatorname{sex}$ & Coll. & Coll.Reg. & figshare DOI for wing images \\
\hline Coenagrionidae & Ischnura pruinescens & $\mathrm{F}$ & $\mathrm{AM}$ & K305381 & https://doi.org/10.6084/m9.figshare. 12591860 \\
\hline Coenagrionidae & Ischnura pruinescens & M & $\mathrm{AM}$ & K305662 & https://doi.org/10.6084/m9.figshare. 12591860 \\
\hline Isostictidae & Labidiosticta vallisi & $\mathrm{F}$ & ANIC & $7-012205$ & https://doi.org/10.6084/m9.figshare. 12591842 \\
\hline Isostictidae & Labidiosticta vallisi & M & $\mathrm{AM}$ & K301412 & https://doi.org/10.6084/m9.figshare. 12591842 \\
\hline Libellulidae & Lathrecista asiatica & $\mathrm{F}$ & $\mathrm{AM}$ & K299562 & https://doi.org/10.6084/m9.figshare.12599945 \\
\hline Libellulidae & Lathrecista asiatica & M & $\mathrm{AM}$ & K299560 & https://doi.org/10.6084/m9.figshare.12599945 \\
\hline Austrocorduliidae & Lathrocordulia garrisoni & M & ANIC & $7-010330$ & https://doi.org/10.6084/m9.figshare.12599942 \\
\hline Austrocorduliidae & Lathrocordulia metallica & $\mathrm{F}$ & ANIC & $7-010343$ & https://doi.org/10.6084/m9.figshare.12599939 \\
\hline Austrocorduliidae & Lathrocordulia metallica & M & $\mathrm{AM}$ & K337589 & https://doi.org/10.6084/m9.figshare.12599939 \\
\hline Lestidae & Lestes concinnus & $\mathrm{F}$ & $\mathrm{AM}$ & K305601 & https://doi.org/10.6084/m9.figshare.12591833 \\
\hline Lestidae & Lestes concinnus & M & $\mathrm{AM}$ & K302019 & https://doi.org/10.6084/m9.figshare. 12591833 \\
\hline Lestoideidae & Lestoidea barbarae & M & ANIC & $7-002864$ & https://doi.org/10.6084/m9.figshare.12591797 \\
\hline Lestoideidae & Lestoidea brevicauda & $\mathrm{F}$ & $\mathrm{AM}$ & K301659 & https://doi.org/10.6084/m9.figshare. 12591788 \\
\hline Lestoideidae & Lestoidea brevicauda & M & $\mathrm{AM}$ & K301661 & https://doi.org/10.6084/m9.figshare. 12591788 \\
\hline Lestoideidae & Lestoidea conjuncta & $\mathrm{F}$ & $\mathrm{AM}$ & K305608 & https://doi.org/10.6084/m9.figshare.12591785 \\
\hline Lestoideidae & Lestoidea conjuncta & M & $\mathrm{AM}$ & K301646 & https://doi.org/10.6084/m9.figshare.12591785 \\
\hline Lestoideidae & Lestoidea lewisiana & $\mathrm{F}$ & $\mathrm{AM}$ & K301645 & https://doi.org/10.6084/m9.figshare. 12591773 \\
\hline Lestoideidae & Lestoidea lewisiana & M & $\mathrm{AM}$ & K301645 & https://doi.org/10.6084/m9.figshare. 12591773 \\
\hline Isostictidae & Lithosticta macra & $\mathrm{F}$ & ANIC & $7-002813$ & https://doi.org/10.6084/m9.figshare. 12591761 \\
\hline Isostictidae & Lithosticta macra & M & ANIC & $7-002814$ & https://doi.org/10.6084/m9.figshare.12591761 \\
\hline Libellulidae & Macrodiplax cora & $\mathrm{F}$ & $\mathrm{AM}$ & K299578 & https://doi.org/10.6084/m9.figshare.12599933 \\
\hline Libellulidae & Macrodiplax cora & M & $\mathrm{AM}$ & K299575 & https://doi.org/10.6084/m9.figshare. 12599933 \\
\hline Macromiidae & Macromia tillyardi & $\mathrm{F}$ & $\mathrm{AM}$ & K403338 & https://doi.org/10.6084/m9.figshare. 12599930 \\
\hline Macromiidae & Macromia tillyardi & M & $\mathrm{AM}$ & K300147 & https://doi.org/10.6084/m9.figshare. 12599930 \\
\hline Macromiidae & Macromia viridescens & $\mathrm{F}$ & $\mathrm{AM}$ & K403339 & https://doi.org/10.6084/m9.figshare.12599927 \\
\hline Macromiidae & Macromia viridescens & M & $\mathrm{AM}$ & K259738 & https://doi.org/10.6084/m9.figshare.12599927 \\
\hline Corduliidae & Metaphya tillyardi & M & $\mathrm{AM}$ & K259746 & https://doi.org/10.6084/m9.figshare.12599924 \\
\hline Austrocorduliidae & Micromidia atrifrons & $\mathrm{F}$ & $\mathrm{AM}$ & K300160 & https://doi.org/10.6084/m9.figshare.12599921 \\
\hline Austrocorduliidae & Micromidia atrifrons & M & $\mathrm{AM}$ & K300159 & https://doi.org/10.6084/m9.figshare.12599921 \\
\hline Austrocorduliidae & Micromidia convergens & $\mathrm{F}$ & $\mathrm{AM}$ & K300149 & https://doi.org/10.6084/m9.figshare. 12599918 \\
\hline Austrocorduliidae & Micromidia convergens & M & $\mathrm{AM}$ & K259797 & https://doi.org/10.6084/m9.figshare. 12599918 \\
\hline Austrocorduliidae & Micromidia rodericki & $\mathrm{F}$ & ANIC & $7-010408$ & https://doi.org/10.6084/m9.figshare. 12599912 \\
\hline Austrocorduliidae & Micromidia rodericki & M & ANIC & 7-010408 & https://doi.org/10.6084/m9.figshare. 12599912 \\
\hline Megapodagrionidae & Miniargiolestes minimus & $\mathrm{F}$ & $\mathrm{AM}$ & K339021 & https://doi.org/10.6084/m9.figshare. 12591758 \\
\hline Megapodagrionidae & Miniargiolestes minimus & M & $\mathrm{AM}$ & K337859 & https://doi.org/10.6084/m9.figshare. 12591758 \\
\hline Libellulidae & Nannodiplax rubra & $\mathrm{F}$ & $\mathrm{AM}$ & K299580 & https://doi.org/10.6084/m9.figshare. 12599909 \\
\hline Libellulidae & Nannodiplax rubra & M & $\mathrm{AM}$ & K299593 & https://doi.org/10.6084/m9.figshare.12599909 \\
\hline Libellulidae & Nannophlebia eludens & $\mathrm{F}$ & $\mathrm{AM}$ & K305515 & https://doi.org/10.6084/m9.figshare. 12599906 \\
\hline Libellulidae & Nannophlebia eludens & M & $\mathrm{AM}$ & K299655 & https://doi.org/10.6084/m9.figshare. 12599906 \\
\hline Libellulidae & Nannophlebia injibandi & $\mathrm{F}$ & MAGNT & I001819 & https://doi.org/10.6084/m9.figshare. 12599903 \\
\hline Libellulidae & Nannophlebia injibandi & M & MAGNT & $\mathrm{I} 001818$ & https://doi.org/10.6084/m9.figshare. 12599903 \\
\hline Libellulidae & Nannophlebia mudginberri & $\mathrm{F}$ & MAGNT & $\mathrm{I} 001821$ & https://doi.org/10.6084/m9.figshare. 12599897 \\
\hline Libellulidae & Nannophlebia mudginberri & M & MAGNT & $\mathrm{I} 001822$ & https://doi.org/10.6084/m9.figshare. 12599897 \\
\hline Libellulidae & Nannophlebia risi & $\mathrm{F}$ & $\mathrm{AM}$ & K299646 & https://doi.org/10.6084/m9.figshare.12599894 \\
\hline Libellulidae & Nannophlebia risi & M & $\mathrm{AM}$ & K299639 & https://doi.org/10.6084/m9.figshare.12599894 \\
\hline Libellulidae & Nannophya australis & $\mathrm{F}$ & $\mathrm{AM}$ & K299607 & https://doi.org/10.6084/m9.figshare. 12599891 \\
\hline Libellulidae & Nannophya australis & M & $\mathrm{AM}$ & K299625 & https://doi.org/10.6084/m9.figshare.12599891 \\
\hline Libellulidae & Nannophya dalei & $\mathrm{F}$ & $\mathrm{AM}$ & K299604 & https://doi.org/10.6084/m9.figshare. 12599888 \\
\hline Libellulidae & Nannophya dalei & M & $\mathrm{AM}$ & K299601 & https://doi.org/10.6084/m9.figshare. 12599888 \\
\hline Libellulidae & Nannophya occidentalis & $\mathrm{F}$ & ANIC & $7-014675$ & https://doi.org/10.6084/m9.figshare.12599885 \\
\hline Libellulidae & Nannophya occidentalis & M & ANIC & $7-014670$ & https://doi.org/10.6084/m9.figshare. 12599885 \\
\hline Libellulidae & Nannophya paulsoni & $\mathrm{F}$ & ANIC & $7-014658$ & https://doi.org/10.6084/m9.figshare. 12599882 \\
\hline Libellulidae & Nannophya paulsoni & M & ANIC & $7-014658$ & https://doi.org/10.6084/m9.figshare. 12599882 \\
\hline Isostictidae & Neosticta canescens & $\mathrm{F}$ & $\mathrm{AM}$ & K301390 & https://doi.org/10.6084/m9.figshare. 12591752 \\
\hline Isostictidae & Neosticta canescens & M & $\mathrm{AM}$ & K301409 & https://doi.org/10.6084/m9.figshare. 12591752 \\
\hline Isostictidae & Neosticta fraseri & $\mathrm{F}$ & $\mathrm{AM}$ & K305621 & https://doi.org/10.6084/m9.figshare. 12591749 \\
\hline Isostictidae & Neosticta fraseri & M & $\mathrm{AM}$ & K301374 & https://doi.org/10.6084/m9.figshare.12591749 \\
\hline Isostictidae & Neosticta silvarum & M & ANIC & $7-002874$ & https://doi.org/10.6084/m9.figshare. 12591710 \\
\hline Calopterygidae & Neurobasis australis & $\mathrm{F}$ & ANIC & unregistered & https://doi.org/10.6084/m9.figshare.12591671 \\
\hline Calopterygidae & Neurobasis australis & M & $\mathrm{AM}$ & K403346 & https://doi.org/10.6084/m9.figshare.12591671 \\
\hline Libellulidae & Neurothemis oligoneura & $\mathrm{F}$ & $\mathrm{AM}$ & K299595 & https://doi.org/10.6084/m9.figshare. 12599879 \\
\hline Libellulidae & Neurothemis oligoneura & M & $\mathrm{AM}$ & K299594 & https://doi.org/10.6084/m9.figshare. 12599879 \\
\hline Libellulidae & Neurothemis stigmatizans & $\mathrm{F}$ & $\mathrm{AM}$ & K299690 & https://doi.org/10.6084/m9.figshare. 12599876 \\
\hline Libellulidae & Neurothemis stigmatizans & M & $\mathrm{AM}$ & K299687 & https://doi.org/10.6084/m9.figshare. 12599876 \\
\hline Platycnemididae & Nososticta baroalba & $\mathrm{F}$ & $\mathrm{AM}$ & K403356 & https://doi.org/10.6084/m9.figshare. 12591617 \\
\hline Platycnemididae & Nososticta baroalba & M & $\mathrm{AM}$ & K403355 & https://doi.org/10.6084/m9.figshare. 12591617 \\
\hline Platycnemididae & Nososticta coelestina & $\mathrm{F}$ & $\mathrm{AM}$ & K301320 & https://doi.org/10.6084/m9.figshare. 12588146 \\
\hline Platycnemididae & Nososticta coelestina & M & $\mathrm{AM}$ & K301316 & https://doi.org/10.6084/m9.figshare. 12588146 \\
\hline Platycnemididae & Nososticta fraterna & $\mathrm{F}$ & ANIC & $7-002456$ & https://doi.org/10.6084/m9.figshare. 12588125 \\
\hline Platycnemididae & Nososticta fraterna & M & $\mathrm{AM}$ & K301312 & https://doi.org/10.6084/m9.figshare.12588125 \\
\hline Platycnemididae & Nososticta kalumburu & $\mathrm{F}$ & $\mathrm{AM}$ & $\mathrm{K} 301311$ & https://doi.org/10.6084/m9.figshare. 12588110 \\
\hline Platycnemididae & Nososticta kalumburu & M & $\mathrm{AM}$ & K301310 & https://doi.org/10.6084/m9.figshare. 12588110 \\
\hline Platycnemididae & Nososticta koolpinyah & $\mathrm{F}$ & ANIC & $7-002496$ & https://doi.org/10.6084/m9.figshare. 12588101 \\
\hline Platycnemididae & Nososticta koolpinyah & M & ANIC & $7-002508$ & https://doi.org/10.6084/m9.figshare. 12588101 \\
\hline Platycnemididae & Nososticta koongarra & $\mathrm{F}$ & $\mathrm{AM}$ & K301305 & https://doi.org/10.6084/m9.figshare. 12582056 \\
\hline Platycnemididae & Nososticta koongarra & M & ANIC & $7-002528$ & https://doi.org/10.6084/m9.figshare. 12582056 \\
\hline Platycnemididae & Nososticta liveringa & $\mathrm{F}$ & ANIC & $7-002536$ & https://doi.org/10.6084/m9.figshare.12582044 \\
\hline Platycnemididae & Nososticta liveringa & M & ANIC & $7-011947$ & https://doi.org/10.6084/m9.figshare. 12582044 \\
\hline Platycnemididae & Nososticta mouldsi & $\mathrm{F}$ & $\mathrm{AM}$ & K301303 & https://doi.org/10.6084/m9.figshare. 12582017 \\
\hline Platycnemididae & Nososticta mouldsi & M & $\mathrm{AM}$ & K301303 & https://doi.org/10.6084/m9.figshare. 12582017 \\
\hline
\end{tabular}




\begin{tabular}{|c|c|c|c|c|c|}
\hline family & species & sex & Coll. & Coll.Reg. & figshare DOI for wing images \\
\hline Platycnemididae & Nososticta pilbara & $\mathrm{F}$ & $\mathrm{AM}$ & K403322 & https://doi.org/10.6084/m9.figshare.12582005 \\
\hline Platycnemididae & Nososticta pilbara & M & $\mathrm{AM}$ & K301302 & https://doi.org $/ 10.6084 / \mathrm{m} 9$. figshare. 12582005 \\
\hline Platycnemididae & Nososticta solida & $\mathrm{F}$ & $\mathrm{AM}$ & K301298 & https://doi.org/10.6084/m9.figshare.12581978 \\
\hline Platycnemididae & Nososticta solida & M & $\mathrm{AM}$ & K301299 & https://doi.org $/ 10.6084 / \mathrm{m} 9$.figshare. 12581978 \\
\hline Platycnemididae & Nososticta solitaria & $\mathrm{F}$ & $\mathrm{AM}$ & K301262 & https://doi.org/10.6084/m9.figshare.12581972 \\
\hline Platycnemididae & Nososticta solitaria & M & $\mathrm{AM}$ & K301216 & https://doi.org $/ 10.6084 / \mathrm{m} 9$.figshare. 12581972 \\
\hline Platycnemididae & Nososticta taracumbi & $\mathrm{F}$ & ANIC & $7-002518$ & https://doi.org $/ 10.6084 / \mathrm{m} 9$. figshare.12581951 \\
\hline Platycnemididae & Nososticta taracumbi & M & $\mathrm{AM}$ & K301212 & https://doi.org/10.6084/m9.figshare.12581951 \\
\hline Telephlebiidae & Notoaeschna geminata & $\mathrm{F}$ & $\mathrm{AM}$ & K299343 & https://doi.org/10.6084/m9.figshare.12599873 \\
\hline Telephlebiidae & Notoaeschna geminata & M & $\mathrm{AM}$ & K299342 & https://doi.org/10.6084/m9.figshare.12599873 \\
\hline Telephlebiidae & Notoaeschna sagittata & $\mathrm{F}$ & $\mathrm{AM}$ & K299331 & https://doi.org/10.6084/m9.figshare.12599870 \\
\hline Telephlebiidae & Notoaeschna sagittata & M & $\mathrm{AM}$ & K299337 & https://doi.org/10.6084/m9.figshare. 12599870 \\
\hline Libellulidae & Notolibellula bicolor & M & $\mathrm{AM}$ & K299709 & https://doi.org/10.6084/m9.figshare.12599864 \\
\hline Gomphidae & Odontogomphus donnellyi & $\mathrm{F}$ & ANIC & $7-006092$ & https://doi.org/10.6084/m9.figshare.12599861 \\
\hline Gomphidae & Odontogomphus donnellyi & M & ANIC & 7-006094 & https://doi.org/10.6084/m9.figshare.12599861 \\
\hline Isostictidae & Oristicta filicicola & $\mathrm{F}$ & $\mathrm{AM}$ & K301365 & https://doi.org $/ 10.6084 / \mathrm{m} 9$.figshare.12581906 \\
\hline Isostictidae & Oristicta filicicola & M & $\mathrm{AM}$ & K301366 & https://doi.org/10.6084/m9.figshare.12581906 \\
\hline Libellulidae & Orthetrum balteatum & $\mathrm{F}$ & ANIC & $7-013521$ & https://doi.org/10.6084/m9.figshare.12599858 \\
\hline Libellulidae & Orthetrum balteatum & M & $\mathrm{AM}$ & K403368 & https://doi.org/10.6084/m9.figshare.12599858 \\
\hline Libellulidae & Orthetrum boumiera & $\mathrm{F}$ & $\mathrm{AM}$ & K299740 & https://doi.org/10.6084/m9.figshare.12599855 \\
\hline Libellulidae & Orthetrum boumiera & M & $\mathrm{AM}$ & K299741 & https://doi.org/10.6084/m9.figshare.12599855 \\
\hline Libellulidae & Orthetrum caledonicum & $\mathrm{F}$ & AM & K299734 & https://doi.org/10.6084/m9.figshare.12599849 \\
\hline Libellulidae & Orthetrum caledonicum & M & $\mathrm{AM}$ & K305580 & https://doi.org/10.6084/m9.figshare.12599849 \\
\hline Libellulidae & Orthetrum migratum & $\mathrm{F}$ & $\mathrm{AM}$ & K299720 & https://doi.org/10.6084/m9.figshare.12599846 \\
\hline Libellulidae & Orthetrum migratum & M & $\mathrm{AM}$ & K299722 & https://doi.org $/ 10.6084 / \mathrm{m} 9$.figshare.12599846 \\
\hline Libellulidae & Orthetrum sabina & $\mathrm{F}$ & $\mathrm{AM}$ & K305165 & https://doi.org/10.6084/m9.figshare.12599843 \\
\hline Libellulidae & Orthetrum sabina & M & $\mathrm{AM}$ & K305163 & https://doi.org/10.6084/m9.figshare.12599843 \\
\hline Libellulidae & Orthetrum serapia & $\mathrm{F}$ & $\mathrm{AM}$ & K305148 & https://doi.org/10.6084/m9.figshare. 12599840 \\
\hline Libellulidae & Orthetrum serapia & M & $\mathrm{AM}$ & K305151 & https://doi.org/10.6084/m9.figshare. 12599840 \\
\hline Libellulidae & Orthetrum villosovittatum & $\mathrm{F}$ & $\mathrm{AM}$ & K305131 & https://doi.org/10.6084/m9.figshare.12599831 \\
\hline Libellulidae & Orthetrum villosovittatum & M & $\mathrm{AM}$ & K305129 & https://doi.org/10.6084/m9.figshare.12599831 \\
\hline Libellulidae & Pantala flavescens & $\mathrm{F}$ & AM & K305237 & https://doi.org/10.6084/m9.figshare.12599825 \\
\hline Libellulidae & Pantala flavescens & M & $\mathrm{AM}$ & K305335 & https://doi.org/10.6084/m9.figshare.12599825 \\
\hline Synthemistidae & Parasynthemis regina & $\mathrm{F}$ & $\mathrm{AM}$ & K299976 & https://doi.org/10.6084/m9.figshare.12599822 \\
\hline Synthemistidae & Parasynthemis regina & M & AM & K299976 & https://doi.org/10.6084/m9.figshare.12599822 \\
\hline Corduliidae & Pentathemis membranulata & $\mathrm{F}$ & $\mathrm{AM}$ & K300201 & https://doi.org/10.6084/m9.figshare.12599819 \\
\hline Corduliidae & Pentathemis membranulata & M & $\mathrm{AM}$ & K300203 & https://doi.org $/ 10.6084 / \mathrm{m} 9$.figshare.12599819 \\
\hline Petaluridae & Petalura gigantea & $\mathrm{F}$ & $\mathrm{AM}$ & K403337 & https://doi.org/10.6084/m9.figshare.12599813 \\
\hline Petaluridae & Petalura gigantea & M & $\mathrm{AM}$ & K305486 & https://doi.org/10.6084/m9.figshare.12599813 \\
\hline Petaluridae & Petalura hesperia & $\mathrm{F}$ & ANIC & $7-005055$ & https://doi.org/10.6084/m9.figshare.12599807 \\
\hline Petaluridae & Petalura hesperia & M & ANIC & $7-005049$ & https://doi.org/10.6084/m9.figshare.12599807 \\
\hline Petaluridae & Petalura ingentissima & $\mathrm{F}$ & $\mathrm{AM}$ & K403367 & https://doi.org/10.6084/m9.figshare.12599801 \\
\hline Petaluridae & Petalura ingentissima & M & ANIC & $7-005063$ & https://doi.org/10.6084/m9.figshare.12599801 \\
\hline Petaluridae & Petalura litorea & $\mathrm{F}$ & $\mathrm{AM}$ & K456322 & https://doi.org/10.6084/m9.figshare.12599780 \\
\hline Petaluridae & Petalura litorea & M & QM & T176058 & https://doi.org/10.6084/m9.figshare.12599780 \\
\hline Megapodagrionidae & Podopteryx selysi & $\mathrm{F}$ & ANIC & 7-009055 & https://doi.org/10.6084/m9.figshare.12581894 \\
\hline Megapodagrionidae & Podopteryx selysi & M & $\mathrm{AM}$ & K301840 & https://doi.org $/ 10.6084 / \mathrm{m} 9$. figshare.12581894 \\
\hline Libellulidae & Potamarcha congener & $\mathrm{F}$ & $\mathrm{AM}$ & K305206 & https://doi.org/10.6084/m9.figshare.12598652 \\
\hline Libellulidae & Potamarch a congener & M & $\mathrm{AM}$ & K305221 & https://doi.org/10.6084/m9.figshare.12598652 \\
\hline Corduliidae & Procordulia affinis & $\mathrm{F}$ & ANIC & 7-010490 & https://doi.org/10.6084/m9.figshare.12598649 \\
\hline Corduliidae & Procordulia affinis & M & ANIC & 7-010501 & https://doi.org/10.6084/m9.figshare.12598649 \\
\hline Corduliidae & Procordulia jacksoniensis & $\mathrm{F}$ & $\mathrm{AM}$ & K403323 & https://doi.org/10.6084/m9.figshare.12598646 \\
\hline Corduliidae & Procordulia jacksoniensis & M & $\mathrm{AM}$ & K300208 & https://doi.org/10.6084/m9.figshare.12598646 \\
\hline Coenagrionidae & Pseudagrion aureofrons & $\mathrm{F}$ & $\mathrm{AM}$ & K305372 & https://doi.org/10.6084/m9.figshare.12581882 \\
\hline Coenagrionidae & Pseudagrion aureofrons & M & $\mathrm{AM}$ & K305371 & https://doi.org/10.6084/m9.figshare. 12581882 \\
\hline Coenagrionidae & Pseudagrion cingillum & $\mathrm{F}$ & ANIC & 7-000466 & https://doi.org/10.6084/m9.figshare.12581861 \\
\hline Coenagrionidae & Pseudagrion cingillum & M & $\mathrm{AM}$ & K305369 & https://doi.org/10.6084/m9.figshare.12581861 \\
\hline Coenagrionidae & Pseudagrion ignifer & $\mathrm{F}$ & $\mathrm{AM}$ & K305325 & https://doi.org $/ 10.6084 / \mathrm{m} 9$.figshare. 12581843 \\
\hline Coenagrionidae & Pseudagrion ignifer & M & $\mathrm{AM}$ & K305332 & https://doi.org $/ 10.6084 / \mathrm{m} 9$. figshare. 12581843 \\
\hline Coenagrionidae & Pseudagrion jedda & $\mathrm{F}$ & ANIC & 7-000961 & https://doi.org $/ 10.6084 / \mathrm{m} 9$. figshare. 12581840 \\
\hline Coenagrionidae & Pseudagrion jedda & M & ANIC & 7-001043 & https://doi.org $/ 10.6084 / \mathrm{m} 9$. figshare. 12581840 \\
\hline Coenagrionidae & Pseudagrion lucifer & $\mathrm{F}$ & ANIC & $7-000426$ & https://doi.org/10.6084/m9.figshare.12581834 \\
\hline Coenagrionidae & Pseudagrion lucifer & M & $\mathrm{AM}$ & K305318 & https://doi.org/10.6084/m9.figshare.12581834 \\
\hline Coenagrionidae & Pseudagrion microcephalum & $\mathrm{F}$ & $\mathrm{AM}$ & K305303 & https://doi.org/10.6084/m9.figshare.12581765 \\
\hline Coenagrionidae & Pseudagrion microcephalum & M & $\mathrm{AM}$ & K305300 & https://doi.org/10.6084/m9.figshare. 12581765 \\
\hline Pseudocorduliidae & Pseudocordulia circularis & $\mathrm{F}$ & ANIC & 7-010312 & https://doi.org/10.6084/m9.figshare. 12598643 \\
\hline Pseudocorduliidae & Pseudocordulia circularis & M & ANIC & 7-010286 & https://doi.org/10.6084/m9.figshare. 12598643 \\
\hline Pseudocorduliidae & Pseudocordulia elliptica & $\mathrm{F}$ & $\mathrm{AM}$ & K305696 & https://doi.org $/ 10.6084 / \mathrm{m} 9$.figshare. 12598640 \\
\hline Pseudocorduliidae & Pseudocordulia elliptica & M & $\mathrm{AM}$ & K305697 & https://doi.org/10.6084/m9.figshare. 12598640 \\
\hline Libellulidae & Raphismia bispina & M & ANIC & 7-014714 & https://doi.org/10.6084/m9.figshare.12598631 \\
\hline Isostictidae & Rhadinosticta banksi & $\mathrm{F}$ & $\mathrm{AM}$ & K301362 & https://doi.org/10.6084/m9.figshare.12581762 \\
\hline Isostictidae & Rhadinosticta banksi & M & $\mathrm{AM}$ & K301361 & https://doi.org/10.6084/m9.figshare.12581762 \\
\hline Isostictidae & Rhadinosticta simplex & $\mathrm{F}$ & $\mathrm{AM}$ & K301347 & https://doi.org/10.6084/m9.figshare.12581756 \\
\hline Isostictidae & Rhadinosticta simplex & M & $\mathrm{AM}$ & K301352 & https://doi.org/10.6084/m9.figshare.12581756 \\
\hline Chlorocyphidae & Rhinocypha tincta & $\mathrm{F}$ & $\mathrm{AM}$ & K403353 & https://doi.org/10.6084/m9.figshare.12581747 \\
\hline Chlorocyphidae & Rhinocypha tincta & M & $\mathrm{AM}$ & K403352 & https://doi.org/10.6084/m9.figshare.12581747 \\
\hline Libellulidae & Rhodothemis lieftincki & $\mathrm{F}$ & AM & K305199 & https://doi.org/10.6084/m9.figshare.12598628 \\
\hline Libellulidae & Rhodothemis lieftincki & M & $\mathrm{AM}$ & K305200 & https://doi.org/10.6084/m9.figshare. 12598628 \\
\hline Libellulidae & Rhyothemis braganza & $\mathrm{F}$ & $\mathrm{AM}$ & K305192 & https://doi.org/10.6084/m9.figshare.12598583 \\
\hline Libellulidae & Rhyothemis braganza & M & $\mathrm{AM}$ & K305191 & https://doi.org $/ 10.6084 / \mathrm{m} 9$.figshare. 12598583 \\
\hline
\end{tabular}




\begin{tabular}{|c|c|c|c|c|c|}
\hline family & species & sex & Coll. & Coll.Reg. & figshare DOI for wing images \\
\hline Libellulidae & Rhyothemis graphiptera & $\mathrm{F}$ & $\mathrm{AM}$ & K305535 & https://doi.org/10.6084/m9.figshare.12598580 \\
\hline Libellulidae & Rhyothemis graphiptera & M & $\mathrm{AM}$ & K305183 & https://doi.org/10.6084/m9.figshare. 12598580 \\
\hline Libellulidae & Rhyothemis phyllis & $\mathrm{F}$ & $\mathrm{AM}$ & K305178 & https://doi.org/10.6084/m9.figshare.12598577 \\
\hline Libellulidae & Rhyothemis phyllis & M & $\mathrm{AM}$ & K305171 & https://doi.org/10.6084/m9.figshare.12598577 \\
\hline Libellulidae & Rhyothemis princeps & $\mathrm{F}$ & $\mathrm{AM}$ & K305288 & https://doi.org/10.6084/m9.figshare.12598571 \\
\hline Libellulidae & Rhyothemis princeps & M & $\mathrm{AM}$ & K305597 & https://doi.org/10.6084/m9.figshare.12598571 \\
\hline Libellulidae & Rhyothemis resplendens & $\mathrm{F}$ & $\mathrm{AM}$ & K403362 & https://doi.org/10.6084/m9.figshare. 12598565 \\
\hline Libellulidae & Rhyothemis resplendens & M & $\mathrm{AM}$ & K305278 & https://doi.org/10.6084/m9.figshare. 12598565 \\
\hline Telephlebiidae & Spinaeschna tripunctata & $\mathrm{F}$ & $\mathrm{AM}$ & K299327 & https://doi.org/10.6084/m9.figshare.12598562 \\
\hline Telephlebiidae & Spinaeschna tripunctata & M & $\mathrm{AM}$ & K299322 & https://doi.org/10.6084/m9.figshare.12598562 \\
\hline Telephlebiidae & Spinaeschna watsoni & $\mathrm{F}$ & ANIC & $7-006800$ & https://doi.org/10.6084/m9.figshare.12598556 \\
\hline Telephlebiidae & Spinaeschna watsoni & M & ANIC & $7-006799$ & https://doi.org/10.6084/m9.figshare.12598556 \\
\hline Synlestidae & Synlestes selysi & $\mathrm{F}$ & $\mathrm{AM}$ & K301602 & https://doi.org/10.6084/m9.figshare.12581741 \\
\hline Synlestidae & Synlestes selysi & M & $\mathrm{AM}$ & K301601 & https://doi.org/10.6084/m9.figshare.12581741 \\
\hline Synlestidae & Synlestes tropicus & $\mathrm{F}$ & $\mathrm{AM}$ & K301595 & https://doi.org/10.6084/m9.figshare.12581729 \\
\hline Synlestidae & Synlestes tropicus & M & $\mathrm{AM}$ & K301598 & https://doi.org/10.6084/m9.figshare. 12581729 \\
\hline Synlestidae & Synlestes weyersii & $\mathrm{F}$ & $\mathrm{AM}$ & K301571 & https://doi.org/10.6084/m9.figshare.12581702 \\
\hline Synlestidae & Synlestes weyersii & M & $\mathrm{AM}$ & K301561 & https://doi.org/10.6084/m9.figshare.12581702 \\
\hline Synthemistidae & Synthemiopsis gomphomacromioides & $\mathrm{F}$ & $\mathrm{AM}$ & K403365 & https://doi.org/10.6084/m9.figshare. 12598550 \\
\hline Synthemistidae & Synthemiopsis gomphomacromioides & M & $\mathrm{AM}$ & K259782 & https://doi.org/10.6084/m9.figshare. 12598550 \\
\hline Synthemistidae & Synthemis eustalacta & $\mathrm{F}$ & $\mathrm{AM}$ & K299972 & https://doi.org/10.6084/m9.figshare.12598541 \\
\hline Synthemistidae & Synthemis eustalacta & M & $\mathrm{AM}$ & K299962 & https://doi.org/10.6084/m9.figshare.12598541 \\
\hline Synthemistidae & Synthemis tasmanica & $\mathrm{F}$ & $\mathrm{AM}$ & K299947 & https://doi.org/10.6084/m9.figshare. 12598538 \\
\hline Synthemistidae & Synthemis tasmanica & M & $\mathrm{AM}$ & K299945 & https://doi.org/10.6084/m9.figshare. 12598538 \\
\hline Coenagrionidae & Teinobasis rufithorax & $\mathrm{F}$ & $\mathrm{AM}$ & K286223 & https://doi.org/10.6084/m9.figshare.12581684 \\
\hline Coenagrionidae & Teinobasis rufithorax & M & $\mathrm{AM}$ & K305297 & https://doi.org/10.6084/m9.figshare.12581684 \\
\hline Telephlebiidae & Telephlebia brevicauda & $\mathrm{F}$ & $\mathrm{AM}$ & K299424 & https://doi.org/10.6084/m9.figshare.12598532 \\
\hline Telephlebiidae & Telephlebia brevicauda & M & $\mathrm{AM}$ & K299421 & https://doi.org/10.6084/m9.figshare.12598532 \\
\hline Telephlebiidae & Telephlebia cyclops & $\mathrm{F}$ & $\mathrm{AM}$ & K299417 & https://doi.org/10.6084/m9.figshare.12598529 \\
\hline Telephlebiidae & Telephlebia cyclops & M & $\mathrm{AM}$ & K299416 & https://doi.org/10.6084/m9.figshare.12598529 \\
\hline Telephlebiidae & Telephlebia godeffroyi & $\mathrm{F}$ & $\mathrm{AM}$ & K299409 & https://doi.org/10.6084/m9.figshare. 12598526 \\
\hline Telephlebiidae & Telephlebia godeffroyi & M & $\mathrm{AM}$ & K299407 & https://doi.org/10.6084/m9.figshare. 12598526 \\
\hline Telephlebiidae & Telephlebia tillyardi & $\mathrm{F}$ & $\mathrm{AM}$ & K299396 & https://doi.org/10.6084/m9.figshare. 12598520 \\
\hline Telephlebiidae & Telephlebia tillyardi & M & $\mathrm{AM}$ & K299401 & https://doi.org/10.6084/m9.figshare. 12598520 \\
\hline Telephlebiidae & Telephlebia tryoni & $\mathrm{F}$ & $\mathrm{AM}$ & K403341 & https://doi.org/10.6084/m9.figshare.12598517 \\
\hline Telephlebiidae & Telephlebia tryoni & M & $\mathrm{AM}$ & K299386 & https://doi.org/10.6084/m9.figshare.12598517 \\
\hline Telephlebiidae & Telephlebia undia & $\mathrm{F}$ & ANIC & $7-007256$ & https://doi.org/10.6084/m9.figshare.12598514 \\
\hline Telephlebiidae & Telephlebia undia & M & ANIC & $7-006287$ & https://doi.org/10.6084/m9.figshare.12598514 \\
\hline Libellulidae & Tetrathemis irregularis & $\mathrm{F}$ & $\mathrm{AM}$ & K305544 & https://doi.org/10.6084/m9.figshare.12598511 \\
\hline Libellulidae & Tetrathemis irregularis & M & $\mathrm{AM}$ & K305275 & https://doi.org/10.6084/m9.figshare.12598511 \\
\hline Libellulidae & Tholymis tillarga & $\mathrm{F}$ & $\mathrm{AM}$ & K305271 & https://doi.org/10.6084/m9.figshare. 12598505 \\
\hline Libellulidae & Tholymis tillarga & M & $\mathrm{AM}$ & K305260 & https://doi.org/10.6084/m9.figshare. 12598505 \\
\hline Synthemistidae & Tonyosynthemis claviculata & $\mathrm{F}$ & $\mathrm{AM}$ & K299944 & https://doi.org/10.6084/m9.figshare.12598502 \\
\hline Synthemistidae & Tonyosynthemis claviculata & M & $\mathrm{AM}$ & K305705 & https://doi.org/10.6084/m9.figshare. 12598502 \\
\hline Synthemistidae & Tonyosynthemis ofarrelli & $\mathrm{F}$ & $\mathrm{AM}$ & K309683 & https://doi.org/10.6084/m9.figshare.12598499 \\
\hline Synthemistidae & Tonyosynthemis ofarrelli & M & $\mathrm{AM}$ & K299935 & https://doi.org/10.6084/m9.figshare.12598499 \\
\hline Libellulidae & Tramea eurybia & $\mathrm{F}$ & QM & $\mathrm{T} 174436$ & https://doi.org/10.6084/m9.figshare.12598496 \\
\hline Libellulidae & Tramea eurybia & M & AM & K403336 & https://doi.org/10.6084/m9.figshare. 12598496 \\
\hline Libellulidae & Tramea loewii & $\mathrm{F}$ & $\mathrm{AM}$ & K305251 & https://doi.org/10.6084/m9.figshare. 12598493 \\
\hline Libellulidae & Tramea loewii & M & $\mathrm{AM}$ & K305254 & https://doi.org/10.6084/m9.figshare. 12598493 \\
\hline Libellulidae & Tramea propinqua & $\mathrm{F}$ & ANIC & $7-013894$ & https://doi.org/10.6084/m9.figshare. 12598490 \\
\hline Libellulidae & Tramea propinqua & M & $\mathrm{AM}$ & K403369 & https://doi.org/10.6084/m9.figshare. 12598490 \\
\hline Libellulidae & Tramea stenoloba & $\mathrm{F}$ & ANIC & $7-013725$ & https://doi.org/10.6084/m9.figshare. 12598484 \\
\hline Libellulidae & Tramea stenoloba & M & ANIC & $7-013749$ & https://doi.org/10.6084/m9.figshare.12598484 \\
\hline Libellulidae & Urothemis aliena & M & $\mathrm{AM}$ & K305503 & https://doi.org/10.6084/m9.figshare.12598481 \\
\hline Coenagrionidae & Xanthagrion erythroneurum & $\mathrm{F}$ & $\mathrm{AM}$ & K337784 & https://doi.org/10.6084/m9.figshare.12581552 \\
\hline Coenagrionidae & Xanthagrion erythroneurum & $\mathrm{M}$ & $\mathrm{AM}$ & K305296 & https://doi.org/10.6084/m9.figshare. 12581552 \\
\hline Gomphidae & Zephyrogomphus lateralis & $\mathrm{F}$ & ANIC & $7-005804$ & https://doi.org/10.6084/m9.figshare. 12598478 \\
\hline Gomphidae & Zephyrogomphus lateralis & M & $\mathrm{AM}$ & K337646 & https://doi.org/10.6084/m9.figshare. 12598478 \\
\hline Gomphidae & Zephyrogomphus longipositor & $\mathrm{F}$ & ANIC & $7-006095$ & https://doi.org/10.6084/m9.figshare. 12598472 \\
\hline Libellulidae & Zyxomma elgneri & $\mathrm{F}$ & $\mathrm{AM}$ & K305240 & https://doi.org/10.6084/m9.figshare.12598469 \\
\hline Libellulidae & Zyxomma elgneri & M & $\mathrm{AM}$ & K305241 & https://doi.org/10.6084/m9.figshare.12598469 \\
\hline Libellulidae & Zyxomma multinervorum & $\mathrm{F}$ & $\mathrm{AM}$ & K403366 & https://doi.org/10.6084/m9.figshare. 12598460 \\
\hline Libellulidae & Zyxomma petiolatum & $\mathrm{F}$ & $\mathrm{AM}$ & K305238 & https://doi.org/10.6084/m9.figshare.12598454 \\
\hline Libellulidae & Zyxomma petiolatum & M & $\mathrm{AM}$ & K305239 & https://doi.org/10.6084/m9.figshare. 12598454 \\
\hline
\end{tabular}




\section{Appendix 4-Library volumes}

This project has created a library of five volumes: two volumes are of images of wings at a variable scale, two volumes are of images of wings at a fixed scale, and one volume is an index.

High resolution images from Volumes 3 and 4 have been downsampled and offered in the present work as an easy-to-view contact-sheet or thumbnail-series (see Appendix 1 for Anisoptera thumbnail-images, pp. 11-63; and Appendix 2 for Zygoptera thumbnail-images, pp. 64-92). Volumes 1-4 (Tann, 2020a-d) hold full sets of images which can be browsed and downloaded individually or as a complete set. The full Index (see Tann, 2020e, abridged in Appendix 3) is a downloadable spreadsheet with links to wing images of each individual species, a whole-of-body image of each reference specimen used, and its occurrence record on the Atlas of Living Australia.

\section{Volume 1}

Anisoptera wings — variable scale lengths, all wings presented at full width Tann, John. 2020a. Wings of Australian Odonata-Volume 1. Anisoptera. figshare. https://doi.org/10.6084/m9.figshare.11845230

\section{Volume 2}

Zygoptera wings — variable scale lengths, all wings presented at full width

Tann, John. 2020b. Wings of Australian Odonata-Volume 1. Zygoptera. figshare. https://doi.org/10.6084/m9.figshare.12579959

\section{Volume 3}

Anisoptera wings-fixed scale length, all wings presented to scale

Tann, John. 2020c. Wings of Australian Odonata-Volume 3. Anisoptera fixed scale. figshare. https://doi.org/10.6084/m9.figshare.12612038

\section{Volume 4}

Zygoptera wings - fixed scale length, all wings presented to scale

Tann, John. 2020d. Wings of Australian Odonata-Volume 4. Zygoptera fixed scale. figshare. https://doi.org/10.6084/m9.figshare.12612041

\section{Index}

Tann, John. 2020e. Wings of Australian Odonata-Index. figshare https://doi.org/10.6084/m9.figshare.11840013 